DESY $10-230$

\title{
Large-distance and long-time asymptotic behavior of the reduced density matrix in the non-linear Schrödinger model.
}

\author{
K. K. Kozlowski $\left.\right|^{1}$
}

\begin{abstract}
Starting from the form factor expansion in finite volume, we derive the multidimensional generalization of the so-called Natte series for the zero-temperature, time and distance dependent reduced density matrix in the non-linear Schrödinger model. This representation allows one to read-off straightforwardly the long-time/large-distance asymptotic behavior of this correlator. This method of analysis reduces the complexity of the computation of the asymptotic behavior of correlation functions in the so-called interacting integrable models, to the one appearing in free fermion equivalent models. We compute explicitly the first few terms appearing in the asymptotic expansion. Part of these terms stems from excitations lying away from the Fermi boundary, and hence go beyond what can be obtained by using the CFT/Luttinger liquid based predictions.
\end{abstract}

\section{Introduction}

One-dimensional quantum models with a gapless spectrum are believed to be critical at zero temperature. In other words, in these models, the ground state expectation values of products of local operators should decay, for large distances of separation between the operators, as some power-law in the distance. It is also believed that, for a generic class of Hamiltonians, the actual value of the exponents governing this power-law decay, the so-called critical exponents, does not depend on the microscopic details of the interactions in the model, but only on its overall symmetries [21, 24]. Therefore two models belonging to the same universality class should be characterized by the same critical exponents.

It has been argued that the equal-time correlation functions in quantum critical one-dimensional models exhibit conformal invariance in the large-distance regime [74]. Hence, its appears plausible to infer their large-distance asymptotics from those of the associated conformal field theory (CFT). The central charge of the CFT lying in the universality class of the model can be deduced from the finite-size corrections to the ground state energy [3, 11]. The possibility to compute such finite-size corrections for many integrable models allowed the identification of the central charge and scaling dimensions leading to the predictions for the critical exponents [5, 17, 18, 54, 55, 84]

${ }^{1}$ DESY, Hamburg, Deutschland, karol.kajetan.kozlowski@ desy.de 
of the long-distance asymptotics. We remind that it is also sometimes possible to give predictions for the critical exponents [25, 26, 67] by putting the model in correspondence with a Luttinger liquid [27].

Due to their wide applicability and relative simplicity, it is more than desirable to test these CFT/Luttinger liquid based predictions versus some exact calculations carried out on such models; this starting from first principle and in such a way that no approximation (apart from assuming a large distance) is made to the very end. Such exact computations have been carried out in the 70's and 80's on various two-point functions appearing in free fermion equivalent model such as the Ising [13, 70, 71], the XY model at the critical magnetic field [68, 69] or the impenetrable Bose gas [83]. The latter approaches were then made much more systematic (and also simplified) with the occurrence of a Riemann-Hilbert based approach to free-fermion models' asymptotics [30] together with the development of the non-linear steepest-descent method [16]. Indeed, the latter constitutes a relatively simple and systematic tool for carrying out the asymptotic analysis [29, 31, 32] of Riemann-Hilbert problems associated with Fredholm determinants representing the correlators in free fermionic models.

However, obtaining long-distance asymptotic expansions of two-point functions for models not equivalent to free fermions faced several additional problems of technical nature. This fact takes its roots in that even obtaining explicit expressions for the correlation functions in the so-called interacting integrable models demands to overcome new types of combinatorial intricacy that disappears when dealing with free fermion equivalent models. The first approach to the problem of computing correlators out of the free fermion point can be attributed to Izergin and Korepin [35, 36]. These authors managed to construct certain series representations for the correlation functions of the non-linear Schrödinger model and the XXZ spin-1/2 chain. However, the $n^{\text {th }}$ summand appearing in these series was only defined implicitly by induction. Low- $n$ calculation allowed them for an effective perturbative characterization of a vicinity of the free fermion point. First manageable expressions for correlators at zero temperature in an interacting integrable model were obtained by Jimbo, Miki, Miwa, Nakayashiki through the vertex operator approach. They have provided multiple integral representations for the matrix elements of the so-called elementary blocks] in the massive [39] regime of the infinite XXZ chain. These results where later extended to the massless regime of this chain [40] or to a half-infinite chain subject to a longitudinal magnetic field acting on one of its ends [38]. The multiple integral representations were then reproduced, in the framework of the algebraic Bethe Ansatz by Kitanine, Maillet and Terras for the massive and massless regime of the periodic XXZ chain [52]. These two series of works opened a way towards a systematic and effective computation of various types of multiple integral and/or combinatorial representations for the correlation functions in numerous integrable models. In particular, it was possible to derive effective representations in the case of finite temperature [23], non-equal times [49], models in finite volume [37], higher spin chains [15],... These results should be seen as of uttermost importance from the conceptual point of view: the multiple integral representations for the correlators of interacting integrable models naturally provides an interpretation for these objects as a new class of special functions (of the distance, time, coupling constants, ...). However, the complexity of the integrands appearing in such multiple integral representations makes the thorough description (computation at certain specific values of the distance/coupling or extracting their large-distance/long-time behavior etc...) of these new special functions a quite challenging problem. Many investigations that followed where oriented towards a better understanding of these special functions. In particular, it was observed that the multiple integral representations for the elementary blocks of the XXZ chain can be reduced to one dimensional integrals by a case-by-case analysis [10, 47, 50, 75]. This observation led to the proof that it is possible to separate the multiple integrals representing the elementary blocks of the XXZ chain on the algebraic level [7]. In its turn, this led to the discovery of a Grassmann structure in the XXZ chain [6, 8, 9]. Among many other developments such as the possibility to compute the one-point functions of the sine-Gordon model [41, 42], the existence of such a Grassmann structure constitutes a promising direction towards bringing the complexity of the analysis of the correlation function of the XXZ chain to the one of a free fermion problem. A completely other method for reducing the complexity of the multiple integral repre-

\footnotetext{
${ }^{\dagger}$ these constitute a basis on which it is possible to decompose all correlation functions of the model
} 
sentations for the correlation functions was the so-called dual field approach [59]. It led to representations for the correlators in terms of expectation values in an infinite dimensional Hilbert space of unbounded operator valued Fredholm determinants [56, 57]. However, apart from convergence issues posed by such an infinite dimensional framework, the main problem of that method was posed by the non-commutativity of the dual field's vacuum expectation values and the asymptotic expansion of a dual-field valued Fredholm determinant. Its and Slavnov [33] carried out, on a formal level, such a dual field-based analysis for the large-distance/long-time decay of the so-called one-particle reduced density matrix at finite temperature in the non-linear Schrödinger model (NLSM). They have been able to provide operator valued expressions for the correlation length. The dual field vacuum expectation values where computed in [80], which led to a description of the correlation length in terms of a solution to a non-linear integral equation. We would like to mention that until recently, although formal, the dual field approach was the only approach alternative to a CFT/Luttinger liquid based correspondence that allowed one to write down certain predictions for the critical exponents.

There have also been developments aiming at obtaining alternative types of effective series of multiple integral representations for the distance dependent two-point functions [48, 51]. The guideline being a construction of such a representation that would allow one to carry out a long-distance asymptotic analysis of the two-point functions. This project has met a success in [45]. This article developed a first fairly rigorous method allowing one to compute, starting from "first principles", the long-distance asymptotic behavior of the spin-spin correlation function in the massless regime of the XXZ spin-1/2 chain. This method relied on a few conjectures relative to the permutation of symbols, summability of the remainders, convergence of the obtained series representations, but was rigorous otherwise. These last results not only confirmed the CFT/Luttinger liquid-based predictions for the critical exponents in this model but also provided explicit expressions for the amplitudes in front of the power-law which, in their turn, cannot be predicted by universality arguments. These explicit formulae for the amplitudes were then identified with certain, properly normalized in respect to the size of the system, form factors of the spin operators [44, 46]. This identification allowed one to point out the universality in the power of the systemsize that one uses for normalizing the form factor associated with the amplitudes. The aforementioned method of asymptotic analysis was applied recently to study the long-distance asymptotic behavior of the correlation functions at finite temperature in the NLSM [63].

The large-distance/long-time asymptotic behavior of the correlation functions in massless one dimensional quantum models goes beyond the predictions stemming from a correspondence with a CFT/Luttinger liquid. Hence, this constitutes a clear motivation for obtaining such an asymptotic behavior from exact considerations on some integrable model as this could help to understand their structure and origin in the general case where exact computations are not feasible. We would like to mention that there already exists several exact results relative to this regime of the asymptotics in the case of free fermion equivalent models [32, 68, 69, 72]. We also would like to remind that there has been proposed recently [28] a non-linear Luttinger liquid theory allowing one to predict the behavior of dynamic structure factors or spectral functions at low energy and momentum. Our results support these predictions along with the recently proposed values for the amplitudes of these functions [12] .

This article develops a method allowing one to compute the zero-temperature asymptotic behavior of the correlation functions in integrable models starting from the form factor expansion for two-point functions. The fact that we build our method on the form factor expansion allows us to include the time-dependence and hence access to the large-distance and long-time asymptotic behavior. The method has been introduced recently on the example of the current-current correlators [65]. Here, we provide many elements of rigor to the method and treat the example of the one particle reduced density matrix in the non-linear Schrödinger model. We would like to stress that this method of asymptotic analysis not only allows us to carry out the analysis in the largedistance/long-time regime but also constitutes an important technical and computational simplification of the approach proposed in [45]. It has also the advantage of being applicable to a much wider class of algebraic Bethe Ansatz solvable models as it solely relies on the universal structure of the form factors in these models. All the 
more than the number of models where these have been determined is constantly growing [14, 36, 53, 61, 73]. The main result of this paper can be summarized as follows. We provide a method for constructing a new type of series representation for the correlation functions of integrable models, that we call multidimensional Natte series. This representation is THE one that is fit for an asymptotic analysis, as the first few terms of the asymptotic expansion can be simply read-off without any effort by looking at the terms of the series. Moreover, the computation of the higher order asymptotics effectively boils down to the case of a free fermionic model (ie computation of subleading asymptotics of the Fredholm determinant of an integrable integral operator) and thus bears the same combinatorial complexity. The main implication of our result for physics is that the asymptotics in the time-dependent case are not only driven by excitations on the Fermi boundary (the latter coincides with the region of the spectrum that can be taken into account by using CFT/Luttinger liquid-based predictions), but also by excitations around the saddle-point $\lambda_{0}$ of the "plane-wave" combination $x p(\lambda)-t \varepsilon(\lambda)$ of the dressed momentum $p$ and dressed energy $\varepsilon$ of the excitations. Also, we provide explicit expressions and identify the associated amplitudes with the infinite volume limit of the properly normalized in the size of the system form factors of the field.

We stress that although we have been able to set our method in a more rigorous framework then it was done in [45, 63], we still have to rely on a few conjectures. More precisely, we have been able to split the asymptotic analysis part from the one of proving the convergence of certain series of multiple integrals representing the correlators. The part related to asymptotic analysis has been set into a rigorous framework. However, in order to raise the results of this asymptotic analysis to the level of the two-point function of interest, we still need to assume on the convergence of the series of multiple integrals we obtain.

The main novelty of this method is that it provides a systematic way for carrying out the asymptotic analysis of multiple integrals or series thereof whose integrands contains some large-parameter dependent driving term being dressed up by coupled functions of the integration variables. We provide a setting that allows one to interpret the "coupled" case as some deformation of the "uncoupled" one. This deformation is such that, provided one is able to carry out the analysis in the "uncoupled" case (but with a sufficiently rich range of functions involved), one is able to deform the "uncoupled" asymptotics back to the "coupled" case of interest. It is in this respect that the analysis carried out in this article strongly relies on the results obtained in [62] (where the relevant "uncoupled" series of multiple integrals of interest has been analyzed) as well as on the fact that correlation functions of generalized free fermionic models (which correspond to the "uncoupled" case) are naturally representable in terms of Fredholm determinants [60].

This paper is organized as follows. In section 2, we remind the definition and main properties of the model. We also introduce all the necessary notations allowing us to present the asymptotic behavior of the reduced density matrix. In section 3, we present our result and discuss the strategy of our method. Then, in section 4, we outline the main properties of the form factors in the model and write down the form factor series for the reduced density matrix. We explain how this series can be re-summed into the so-called multidimensional deformation of the Natte series. Once that such a representation is built, thanks to the very properties of the Natte series, it is possible to literally read-off the first few terms of the asymptotic expansion. We gather all the auxiliary and technical results in several appendices. We discuss the large size-behavior of the form factors of the fields in appendix A In appendix B, we derive finite-size Fredholm minor representations for the form factor based expansions of certain two-point functions in generalized free fermion models. In appendix $\mathrm{C}$, we prove the existence of the thermodynamic (ie infinite volume) limit for certain quantities of interest. We also provide various alternative expressions for this limit. In appendix D we develop the theory of functional translation in spaces of holomorphic functions. The results established in this appendix constitute the main tools of our analysis. They allow for an effective separation of variables in the intermediate steps so that one is able to carry out various re-summations of the formulae by building on the results stemming from the generalized free fermion model studied in appendix B 


\section{The non-linear Schrödinger model}

\subsection{The eigenstates and Bethe equations}

The non-linear Schrödinger model corresponds to the Hamiltonian

$$
\boldsymbol{H}_{N L S}=\int_{0}^{L}\left\{\partial_{y} \Phi^{\dagger}(y) \partial_{y} \Phi(y)+c \Phi^{\dagger}(y) \Phi^{\dagger}(y) \Phi(y) \Phi(y)-h \Phi^{\dagger}(y) \Phi(y)\right\} \mathrm{d} y .
$$

The model is defined on a circle of length $L$, so that the canonical Bose fields $\Phi, \Phi^{\dagger}$ are subject to $L$-periodic boundary conditions. In the following, we will focus on the repulsive regime $c>0$ in the presence of a positive chemical potential $h>0$. The Hamiltonian $\boldsymbol{H}_{N L S}$ commutes with the number of particles operator, and thus can be diagonalized independently in every sector with a fixed number of particles $N$. In each of these sectors, the model is equivalent to a $\mathrm{N}$-body gas of bosons subject to $\delta$-like repulsive interactions. The corresponding model of interacting bosons was first proposed and studied by Girardeau [22] in the $c=+\infty$ case and then introduced and solved, through the coordinate Bethe Ansatz, by Lieb and Liniger [66] in the case of arbitrary $c$. It is also possible to build the eigenstates of the Hamiltonian by means of the algebraic Bethe Ansatz. This was first done by Sklyanin [77] directly in the infinite volume. In the case of finite volume $L$, as observed by Izergin and Korepin [34], it is possible to put the continuous model on a lattice in such a way that the standard construction [20] of the algebraic Bethe Ansatz holds. At the end of the computations, it is then possible to send the lattice spacing to zero and recover the spectrum and eigenstates of the continuous model. The fact that this manipulation is indeed fully rigorous has been shown by Dorlas [19].

In the algebraic Bethe Ansatz approach, the Hamiltonian (2.1) appears as a member of a one-parameter commuting family of operators $\lambda \mapsto \mathcal{T}(\lambda)$. It is sometimes useful to consider a $\beta$-deformation of this family $\mathcal{T}_{\beta}(\lambda)$, such that $\mathcal{T}_{\beta}(\lambda)_{\mid \beta=0}=\mathcal{T}(\lambda)$. The common eigenstates $\left|\psi_{\beta}(\{\mu\})\right\rangle$ of $\mathcal{T}_{\beta}(\lambda)$ in the $N_{\kappa}$-particle sector are parameterized by a set of real numbers $\left\{\mu_{\ell_{a}}\right\}_{a=1}^{N_{k}}$ which are the unique solutions to the $\beta$-deformed logarithmic Bethe equations [4, 85]

$$
L p_{0}\left(\mu_{\ell_{a}}\right)+\sum_{b=1}^{N_{K}} \theta\left(\mu_{\ell_{a}}-\mu_{\ell_{b}}\right)=2 \pi\left(\ell_{a}-\frac{N_{\kappa}+1}{2}\right)+2 i \pi \beta \quad \text { with } \quad p_{0}(\lambda)=\lambda \quad \text { and } \quad \theta(\lambda)=i \ln \left(\frac{i c+\lambda}{i c-\lambda}\right) .
$$

$p_{0}$ is called the bare momentum and $\theta$ the bare phase. The set of solutions corresponding to all choices of integers $\ell_{a} \in \mathbb{Z}$ such that $\ell_{1}<\cdots<\ell_{N_{\kappa}}$ yield the complete set of eigenstates in the $N_{\kappa}$-particle sector [19].

In each sector with a fixed number of particles $N_{\kappa}$, the so-called ground state's Bethe roots are given by the solution to (2.2) corresponding to the choice of $N_{K}$ consecutive integers $\ell_{a}=a$, with $a=1, \ldots, N_{K}$ and $\beta=0$. The number $N_{K}$ corresponding to the number of particles in the overall ground state of $\boldsymbol{H}_{N L S}$ is imposed by the chemical potential $h$ and scales with $L$. It will be denoted by $N$ in the following. One shows that in the thermodynamic limit $(N, L \rightarrow+\infty$ so that $N / L \rightarrow D)$ the parameters $\left\{\lambda_{j}\right\}_{1}^{N}$ associated to this ground state condensate on a symmetric interval $[-q ; q]$ called the Fermi zone.

All other choices of sets of integers $\ell_{a}$ lead to ( $\beta$-deformed) excited states. In principle, these excited states can also be found in sectors with a different number $N_{\kappa} \neq N$ of particles. It is convenient to describe the excited states in the language of particle-hole excitations above the $N_{\kappa}$-particle $\beta$-deformed ground state 7 . Namely, such an excited state corresponds to a choice of integers $\ell_{j}$ in (2.2) such that

$$
\ell_{j}=j \quad \text { for } j \in \llbracket 1 ; N_{\kappa} \rrbracket \backslash h_{1}, \ldots, h_{n} \quad \text { and } \quad \ell_{h_{a}}=p_{a} \quad \text { for } a=1, \ldots, n .
$$

\footnotetext{
${ }^{\dagger}$ the $\beta$-deformed ground state corresponds to the choice $\ell_{a}=a$, with $a=1, \ldots, N_{\kappa}$
} 
The integers $p_{a}$ and $h_{a}$ are such that $p_{a} \notin \llbracket 1 ; N_{\kappa} \rrbracket \equiv\left\{1, \ldots, N_{\kappa}\right\}$ and $h_{a} \in \mathbb{[} 1 ; N_{\kappa} \rrbracket$. There is thus a one-to-one correspondence between integers $\ell_{j}$ and the integers $h_{a}$ and $p_{a}$ describing particle-hole excitations.

In this picture, the integers $h_{a}$ correspond to holes in the increasing sequence of integers defining the $\beta$ deformed ground state roots, whereas $p_{a}$ correspond to extra integers appearing in the equation and can be seen as defining some new position of "particles". Given a solution $\left\{\mu_{\ell_{a}}\right\}_{1}^{N_{\kappa}}$ corresponding to a fixed choice of integers $\ell_{1}<\cdots<\ell_{N_{K}}$ it is convenient to introduce their counting function:

$$
\widehat{\xi}_{\left\{\ell_{a}\right\}}(\omega) \equiv \widehat{\xi}_{\left\{\ell_{a}\right\}}\left(\omega \mid\left\{\mu_{\ell_{a}}\right\}_{1}^{N_{\kappa}}\right)=\frac{p_{0}(\omega)}{2 \pi}+\frac{1}{2 \pi L} \sum_{a=1}^{N_{K}} \theta\left(\omega-\mu_{\ell_{a}}\right)+\frac{N_{K}+1}{2 L}-i \frac{\beta}{L}
$$

By construction, it is such that $\widehat{\xi}_{\left\{\ell_{a}\right\}}\left(\mu_{f_{a}}\right)=\ell_{a} / L$, for $a=1, \ldots, N_{\kappa}$. Actually, $\widehat{\xi}_{\left\{\ell_{a}\right\}}(\omega)$ defines $\mathbb{\Psi}$ a set of background parameters $\left\{\mu_{a}\right\}, a \in \mathbb{Z}$, as the unique solutions to $\widehat{\xi}_{\left\{\ell_{a}\right\}}\left(\mu_{a}\right)=a / L$. The latter allows one to define the rapidities $\mu_{p_{a}}$, resp. $\mu_{h_{a}}$, of the particles, resp. holes, entering in the description of $\left\{\mu_{\ell_{a}}\right\}_{1}^{N_{K}}$.

\subsection{The thermodynamic limit}

When the thermodynamic limit of the model is considered, it is possible to provide a slightly more precise description of the solution to the Bethe equations for the ground state $\left\{\lambda_{a}\right\}_{a=1}^{N}$ as well as for any particle-hole type $\beta$-deformed excited states $\left\{\mu_{\ell_{a}}\right\}_{a=1}^{N_{k}}$ above it with $N_{k}-N$ being fixed and not depending on $L$ or $N$. Introducing the counting function for the ground state

$$
\widehat{\xi}(\omega) \equiv \widehat{\xi}\left(\omega \mid\left\{\lambda_{a}\right\}_{1}^{N}\right)=\frac{p_{0}(\omega)}{2 \pi}+\frac{1}{2 \pi L} \sum_{a=1}^{N_{K}} \theta\left(\omega-\lambda_{a}\right)+\frac{N+1}{2 L}, \quad \text { ie } \widehat{\xi}\left(\lambda_{a}\right)=\frac{a}{L},
$$

it can be shown that, in the thermodynamic limit, it behaves as

$$
\widehat{\xi}(\omega)=\xi(\omega)+\mathrm{O}\left(L^{-1}\right) \quad \text { where } \quad \xi(\omega)=\frac{p(\omega)}{2 \pi}+\frac{D}{2} \quad \text { and } \quad N / L \rightarrow D .
$$

There, the $\mathrm{O}\left(L^{-1}\right)$ is uniform and holomorphic in $\omega$ belonging to a strip of some fixed width around the real axis, $p$ is the so-called dressed momentum, defined as the unique solution to the integral equation

$$
p(\lambda)-\int_{-q}^{q} \theta(\lambda-\mu) p^{\prime}(\mu) \frac{\mathrm{d} \mu}{2 \pi}=p_{0}(\lambda)
$$

The parameter $q$ corresponds to the right end of the Fermi interval $[-q ; q]$ on which the ground state's Bethe roots condensate. It is fixed by the value of the chemical potential $h$ by demanding that the dressed energy $\varepsilon(\lambda)$, defined as the unique solution to the below integral equation, vanishes at $\pm q$ :

$$
\varepsilon(\lambda)-\int_{-q}^{q} K(\lambda-\mu) \varepsilon(\mu) \frac{\mathrm{d} \mu}{2 \pi}=\varepsilon_{0}(\lambda) \quad \text { with } \varepsilon_{0}(\lambda)=\lambda^{2}-h \quad \text { and } \varepsilon( \pm q)=0 \text {. }
$$

We also remind the relation $p_{F}=\pi D$ where $p_{F}=p(q)$ is the Fermi momentum.

\footnotetext{
${ }^{\dagger}$ Note that different sets of roots $\left\{\mu_{\ell_{a}}\right\}$ and $\left\{\mu_{\ell_{a}^{\prime}}\right\}$ lead to different sets of background parameters

ॠThe uniqueness of solutions follows from the fact that the solution to 2.2 are such that $\mu_{\ell_{a}}(\beta)=\mu_{\ell_{a}}(0)+2 i \pi \beta / L$. This allows one to show that $\widehat{\xi}_{\left\{\ell_{a}\right\}}(\omega)$ is strictly increasing on $\mathbb{R}+2 i \pi \beta / L$ and maps it onto $\mathbb{R}$. Moreover, one can check that $\mathfrak{J}\left(\widehat{\xi}_{\left\{\ell_{a}\right\}}\right) \neq 0$ on $\mathbb{C} \backslash(\mathbb{R}+2 i \pi \beta / L)$.
} 
In the following, we will focus on the excited states in the $N_{\kappa}=N+1$-particle sector only. In order to describe the thermodynamic properties of such $\beta$-deformed excited states, it is convenient to introduce the associated shift function

$$
\widehat{F}_{\left\{\ell_{a}\right\}}(\omega) \equiv \widehat{F}\left(\omega \mid\left\{\mu_{\ell_{a}}\right\}_{1}^{N+1}\right)=L\left[\widehat{\xi}(\omega)-\widehat{\xi}_{\left\{\ell_{a}\right\}}(\omega)\right]=\frac{1}{2 \pi} \sum_{a=1}^{N} \theta\left(\omega-\lambda_{a}\right)-\frac{1}{2 \pi} \sum_{a=1}^{N+1} \theta\left(\omega-\mu_{\ell_{a}}\right)-\frac{1}{2}+i \beta .
$$

It can be shown that this counting function admits a thermodynamic limit $F_{\beta}$ that solves the linear integral equation

$$
F_{\beta}\left(\lambda \mid \begin{array}{l}
\left\{\mu_{p_{a}}\right\} \\
\left\{\mu_{h_{a}}\right\}
\end{array}\right)-\int_{-q}^{q} K(\lambda-\mu) F_{\beta}\left(\mu \mid \begin{array}{l}
\left\{\mu_{p_{a}}\right\} \\
\left\{\mu_{h_{a}}\right\}
\end{array}\right) \frac{\mathrm{d} \mu}{2 \pi}=i \beta-\frac{1}{2}-\frac{1}{2 \pi} \theta(\lambda-q)-\frac{1}{2 \pi} \sum_{a=1}^{n}\left[\theta\left(\lambda-\mu_{p_{a}}\right)-\theta\left(\lambda-\mu_{h_{a}}\right)\right] .
$$

There $\mu_{p_{a}}$, resp. $\mu_{h_{a}}$, are to be understood as the unique solutions to $\xi\left(\mu_{p_{a}}\right)=p_{a} / L$, resp. $\xi\left(\mu_{h_{a}}\right)=h_{a} / L$, where $\xi$ is given by (2.6). Note that we have explicitly insisted on the auxiliary dependence of the thermodynamic limit of the shift function on the positions of the particles/holes. However, in the following, whenever the value of $\left\{\mu_{p_{a}}\right\}$ and $\left\{\mu_{h_{a}}\right\}$ will be dictated by the context, we will omit it. We also remind that the above shift function measures the spacing between the ground state roots $\lambda_{a}$ and the background parameters $\mu_{a}$ defined by $\widehat{\xi}_{\left\{\ell_{a}\right\}}$ : $\mu_{a}-\lambda_{a}=F_{\beta}\left(\lambda_{a}\right) \cdot\left[L \xi^{\prime}\left(\lambda_{a}\right)\right]^{-1}\left(1+\mathrm{O}\left(L^{-1}\right)\right)$.

The integral equation for the thermodynamic limit of the shift function $F_{\beta}$ can be solved in terms of the dressed phase $\phi(\lambda, \mu)$ and dressed charge $Z(\lambda)$

$$
\phi(\lambda, \mu)-\int_{-q}^{q} K(\lambda-\tau) \phi(\tau, \mu) \frac{\mathrm{d} \tau}{2 \pi}=\frac{1}{2 \pi} \theta(\lambda-\mu) \quad \text { and } \quad Z(\lambda)-\int_{-q}^{q} K(\lambda-\tau) Z(\tau) \frac{\mathrm{d} \tau}{2 \pi}=1
$$

Namely,

$$
F_{\beta}(\lambda) \equiv F_{\beta}\left(\lambda \mid \begin{array}{l}
\left\{\mu_{p_{a}}\right\} \\
\left\{\mu_{h_{a}}\right\}
\end{array}\right)=(i \beta-1 / 2) Z(\lambda)-\phi(\lambda, q)-\sum_{a=1}^{n}\left[\phi\left(\lambda, \mu_{p_{a}}\right)-\phi\left(\lambda, \mu_{h_{a}}\right)\right]
$$

Here, we also remind two very nice relationships that exist between the dressed phase and dressed charge

$$
Z(\lambda)=1+\phi(\lambda,-q)-\phi(\lambda, q) \quad \text { and } \quad Z^{-1}(q)=1+\phi(-q, q)-\phi(q, q) .
$$

The first one is easy to obtain and the second one has been obtained in [58, 79].

The shift function allows one to compute many thermodynamic limits involving the parameters $\left\{\mu_{\ell_{a}}\right\}$. For instance, introducing the combination of bare momentum and energy $u_{0}(\lambda)=p_{0}(\lambda)-t \varepsilon_{0}(\lambda) / x$, one readily sees that for a $n$ particle/hole excited state $\left\{\mu_{\ell_{a}}\right\}$ at $\beta=0$

$$
\lim _{N, L \rightarrow+\infty}\left\{\sum_{a=1}^{N+1} u_{0}\left(\mu_{\ell_{a}}\right)-\sum_{a=1}^{N} u_{0}\left(\lambda_{a}\right)\right\}_{\beta=0}=\sum_{a=1}^{n} u\left(\mu_{p_{a}}\right)-u\left(\mu_{h_{a}}\right) .
$$

Above and in the following, $u$ stands for the combination of dressed momenta and energies $u(\lambda)=p(\lambda)-t \varepsilon(\lambda) / x$. It admits the integral representation

$$
u(\lambda)=u_{0}(\lambda)-\int_{-q}^{q} u_{0}^{\prime}(\mu) \phi(\mu, \lambda) \mathrm{d} \mu
$$


The function $u_{0}^{\prime}$ admits a unique zero of first order on $\mathbb{R}$. It is believed that this property is preserved for $u$. Clearly, in virtue of Rouché's theorem, this holds true for $c$ large enough. We will not purse the discussion of this property here as it goes out of the scope of this paper and will use it as a working hypothesis. In other words, we assume that given a fixed ratio $t / x$, there exists a unique $\lambda_{0}$ such that $u^{\prime}\left(\lambda_{0}\right)=0$ and $u^{\prime \prime}\left(\lambda_{0}\right)<0$.

We do stress however that this working hypothesis should not be considered as a restriction but a simplification of the exposition at most. Indeed, it follows from $\left|u^{\prime}(\lambda)\right| \rightarrow+\infty$ when $\mathfrak{R}(\lambda) \rightarrow \pm \infty$ that, for any value of $c>0$, $u^{\prime}$ has a finite number of real zeroes. The case when $u^{\prime}$ has multiple real zeroes of arbitrary order could be treated within out method but would make the analysis heavier.

As a concluding remark to this section, we would like to stress that all functions that have been introduced above (the dressed momentum $p$, the dressed energy $\varepsilon$, the dressed charge $Z$ and the dressed phase $\phi$ ) are holomorphic in the strip

$$
U_{\delta}=\{z \in \mathbb{C}:|\mathfrak{J}(z)|<2 \delta\}
$$

around the real axis. The parameter $\delta$ satisfies $c / 8>\delta>0$ and is chosen sufficiently small so that $p$ is injective on $U_{\delta}$ and $\inf _{\lambda \in U_{\delta}}[\mathfrak{R}(Z(\lambda))]>0$. We will tacitly assume such a choice in the following each time the strip $U_{\delta}$ will be used.

\section{The method and main results}

The zero-temperature one-particle reduced density matrix in finite volume refers to the below ground state expectation value:

$$
\rho_{N}(x, t) \equiv\left\langle\psi\left(\left\{\lambda_{a}\right\}_{1}^{N}\right)\left|\Phi(x, t) \Phi^{\dagger}(0,0)\right| \psi\left(\left\{\lambda_{a}\right\}_{1}^{N}\right)\right\rangle \cdot\left\|\psi\left(\left\{\lambda_{a}\right\}_{1}^{N}\right)\right\|^{-2} .
$$

The parameters $\left\{\lambda_{a}\right\}_{1}^{N}$ correspond to the set of Bethe roots parameterizing the ground state of (2.1). We recall that the fields evolve in space and time according to

$$
\Phi(x, t)=\mathrm{e}^{-i x P+i t \boldsymbol{H}_{N L S}} \Phi(0,0) \mathrm{e}^{i x P-i t \boldsymbol{H}_{N L S}},
$$

where $\boldsymbol{H}_{N L S}$ is the Hamiltonian of the model given in (2.1) and $P$ is the total momentum operator. The action of $P$ on the eigenstates of $\boldsymbol{H}_{N L S}$ has been computed in [4].

We denote by $\rho(x, t)=\lim _{N, L \rightarrow+\infty} \rho_{N}(x, t)$ the, presumably existing, thermodynamic limit of $\rho_{N}(x, t)$. We will not develop further on the existence of this limit, and take this as a quite reasonable working hypothesis.

\subsection{Description of the method}

In this article, we carry out several manipulations that lead us to propose a series representation for $\rho(x, t)$ giving a straightforward access to its leading large-distance/long-time asymptotic behavior.

The starting point of our analysis is the model in finite volume. We will first provide certain re-summation formulae for $\rho_{N}(x, t)$ starting from the form factor expansion of (3.1). The latter involves a summation over all the excited states (ie over all solutions to (2.2)-(2.3) at $\beta=0$ ). This sum has a very intricate structure which prevents us from analyzing its thermodynamic limit rigorously from the very beginning. We therefore introduce a simplifying hypothesis. Namely, denoting the energy of an excited state by $E_{\mathrm{ex}}$ and the one of the ground state by $E_{\mathrm{gs}}$ we argue that all contributions issued from excited states characterized by $E_{\mathrm{ex}}-E_{\mathrm{gs}}=\mathrm{O}(L)$ do not contribute to the thermodynamic limit of the form factor expansion of $\rho_{N}(x, t)$. In the light of these arguments, we are led to analyze an effective form factor series $\rho_{N \text {; eff }}(x, t)$ and a certain $\gamma$-deformation $\rho_{N ; \text { eff }}(x, t \mid \gamma)$ thereof. Our conjecture is that $\rho_{N ; \text { eff }}(x, t \mid \gamma=1)=\rho_{N ; \text { eff }}(x, t)$ has the same thermodynamic limit as $\rho_{N}(x, t)$. 
We study $\gamma \mapsto \rho_{N \text {;eff }}(x, t \mid \gamma)$ by means of its Taylor coefficients at $\gamma=0$ :

$$
\left.\rho_{N ; \mathrm{eff}}^{(m)}(x, t) \equiv \frac{\partial^{m}}{\partial \gamma^{m}} \rho_{N ; \text { eff }}(x, t \mid \gamma)\right|_{\gamma=0} .
$$

All rigorous, conjecture-free, results of this paper are relative to these Taylor coefficients. We show that these admit a well defined thermodynamic limit $\rho_{\mathrm{eff}}^{(m)}(x, t)$. In addition, we provide two different representations for this limit, each being a finite sum of multiple integrals.

- The first representation is in the spirit of the ones obtained in [45, 63]. It corresponds to some truncation of a multidimensional deformation of a Fredholm series for a Fredholm minor.

- The second representation is structured in such a way that it allows one to read-off straightforwardly the first few terms of the asymptotic expansion of $\rho_{\mathrm{eff}}^{(m)}(x, t)$. The various terms appearing in this representation are organized in such a way that the identification of those that are negligible (eg exponentially small) in the $x \rightarrow+\infty$ limit is trivial.

The above two results are derived rigorously without any approximation or additional conjecture. However, in order to push the analysis a little further and provide results that would have applications to physics, we need to rely on several conjectures. Namely, we assume that

1. the series of multiple integrals that arises upon summing up the thermodynamic limits of the Taylor coefficients $\sum_{m=0}^{+\infty} \rho_{\text {eff }}^{(m)}(x, t) / m$ ! is convergent;

2. this sum moreover coincides with the thermodynamic limit of $\rho_{N ; \text { eff }}(x, t \mid \gamma=1)$ and hence, due to our first conjecture, with $\rho(x, t)$.

These conjectures allow us to claim that $\rho(x, t)$ can be represented in terms of a series of multiple integrals. The latter series corresponds to a multidimensional deformation of the Natte series expansion for Fredholm minors of integrable integral operators [62]. This multidimensional Natte series has all the virtues in respect to the computation of the long-time/large-distance asymptotic behavior of $\rho(x, t)$; it is structured in such a way that one readily reads-off from its very form, the sub-leading and the first few leading terms of the asymptoics.

So as to conclude the description of our method, we would like to stress that the aforementioned conjectures of convergence are supported by the fact that they can be proven to hold in the limiting case of a generalized free fermion model [62]. Unfortunately, the highly coupled nature of the integrands involved in our representations does not allow one for any simple check of the convergence properties in the general $+\infty>c>0$ case.

\subsection{Large-distance/long-time asymptotic behavior of the one-particle reduced density matrix}

We have now introduced enough notations so as to be able to present the physically interesting part of our analysis.

Let $x>0$ be large and the ratio $x / t$ is fixed. Let $\lambda_{0}$ be the associated, presumably unique ( $c f(2.14)$ ), saddlepoint of $u(\lambda)=p(\lambda)-t \varepsilon(\lambda) / x$. Assume in addition that $\lambda_{0} \neq \pm q$ and $\lambda_{0}>-q$. Then, under the validity of the aforementioned conjectures, the thermodynamic limit of the zero-temperature one-particle reduced density matrix 
$\rho(x, t)$ admits the asymptotic expansion

$$
\begin{gathered}
\rho(x, t)=\sqrt{\frac{-2 i \pi}{t \varepsilon^{\prime \prime}\left(\lambda_{0}\right)-x p^{\prime \prime}\left(\lambda_{0}\right)}} \frac{p^{\prime}\left(\lambda_{0}\right) \mathrm{e}^{i x\left[u\left(\lambda_{0}\right)-u(q)\right]}\left|\mathcal{F}_{q}^{\lambda_{0}}\right|^{2}}{\left[i\left(x+v_{F} t\right)\right]^{\left[F_{q}^{\lambda_{0}}(-q)\right]^{2}\left[-i\left(x-v_{F} t\right)\right]^{\left[F_{q}^{\lambda_{0}}(q)\right]^{2}}}\left(\mathbf{1}_{q ;+\infty}\left[\left(\lambda_{0}\right)+\mathrm{o}(1)\right)\right.} \\
+\frac{\mathrm{e}^{-2 i x p_{F}}\left|\mathcal{F}_{q}^{-q}\right|^{2}}{\left[i\left(x+v_{F} t\right)\right]^{\left[F_{q}^{-q}(-q)-1\right]^{2}}\left[-i\left(x-v_{F} t\right)\right]^{\left[F_{q}^{-q}(q)\right]^{2}}}(1+\mathrm{o}(1)) \\
+\frac{\left|\mathcal{F}_{\emptyset}^{\emptyset}\right|^{2}}{\left[i\left(x+v_{F} t\right)\right]^{\left[F_{\emptyset}^{\emptyset}(-q)\right]^{2}}\left[-i\left(x-v_{F} t\right)\right]\left[F_{\emptyset}^{\emptyset}(q)+1\right]^{2}}(1+o(1)) .
\end{gathered}
$$

The critical exponents governing the algebraic decay in the distance of separation are expressed in terms of the thermodynamic limit $F_{\mu_{h}}^{\mu_{p}}$ of the shift function (at $\beta=0$ ) associated with an excited state of (2.1) having one particle at $\mu_{p}$ and one hole at $\mu_{h}$, namely,

$$
F_{\emptyset}^{\emptyset}(\lambda)=-\frac{Z(\lambda)}{2}-\phi(\lambda, q) \quad F_{q}^{-q}(\lambda)=-\frac{Z(\lambda)}{2}-\phi(\lambda,-q) \quad F_{q}^{\lambda_{0}}(\lambda)=-\frac{Z(\lambda)}{2}-\phi\left(\lambda, \lambda_{0}\right) .
$$

The type of algebraic decay in (3.4) can be organized in two classes. There is a square root power-law decay $\left(t \varepsilon^{\prime \prime}\left(\lambda_{0}\right)-x p^{\prime \prime}\left(\lambda_{0}\right)\right)^{-\frac{1}{2}}$ stemming from the saddle-point $\lambda_{0}$. All other sources of algebraic decay appear in the so-called relativistic combinations $x \pm v_{F} t$ and exhibit non-trivial critical exponents driven by the shift function of the underlying type of excitation. We recall that $\pm v_{F}$ corresponds to the velocity of the excitations on the right/left Fermi boundary: $v_{F}=\varepsilon^{\prime}(q) / p^{\prime}(q)$.

Each of the three terms in these leading asymptotics has its amplitude $\left(\left|\mathcal{F}_{q}^{\lambda_{0}}\right|^{2},\left|\mathcal{F}_{\emptyset}^{\emptyset}\right|^{2}\right.$ or $\left.\left|\mathcal{F}_{q}^{-q}\right|^{2}\right)$ given by the thermodynamic limit of properly normalized in the length $L$ moduli squared of form factors of the conjugated field $\Phi^{\dagger}$. More precisely,

- $\left|\mathcal{F}_{q}^{\lambda_{0}}\right|^{2}$ involves the form factor of $\Phi^{\dagger}$ taken between the $N$-particle ground state and an excited state above the $N+1$ particle ground state with one particle at $\lambda_{0}$ and one hole at $q$.

- $\left|\mathcal{F}_{q}^{-q}\right|^{2}$ corresponds to the case when one considers an excited state above the $N+1$-particle ground state with one particle at $-q$ and one hole at $q$.

- $\left|\mathcal{F}_{\emptyset}^{\emptyset}\right|^{2}$ corresponds to the case where the form factor average of $\Phi^{\dagger}$ is taken between the $\mathrm{N}$ and the N+1particle ground state.

The explicit (but rather cumbersome) expressions for the amplitudes together with a more precise definition are postponed to appendix A.3.

Finally, $\mathbf{1}_{q ;+\infty}$ [ stands for the characteristic function of the interval ] $q ;+\infty[$. It is there so as to indicate that, to the leading order, the contribution stemming from the saddle-point only appears in the space-like regime $\lambda_{0}>q$. We stress that by pushing the calculations to an even higher order in $x$, one can show that hole-type excitations in a vicinity of the saddle-point also contribute in the time-like regime where $\left.\lambda_{0} \in\right]-q ; q[$. Their contribution is however much more dampen. 


\section{Remarks}

The oscillating phases and amplitudes appearing in (3.4) are reminiscent of the type of excitations that give rise to their associated contribution. Each term in (3.4) can be associated with some macroscopic state of the model. For instance, the one occuring in the first line of (3.4) corresponds to a macroscopic state characterized by one particle at $\lambda_{0}$ and one hole at $q$. There are infinitely many microscopic realizations of such a macroscopic state. For instance, any excited state realized as one particle at $\lambda_{0}$, one hole at $q$,

- $n_{+}$particles $\mu_{p_{a}}^{(r)}$ and holes $\mu_{h_{a}}^{(r)}$ located at $q$ in the thermodynamic limit: $\mu_{p_{a}}^{(r)}, \mu_{h_{a}}^{(r)} \underset{N, L \rightarrow+\infty}{\longrightarrow} q$ for $a=1, \ldots, n_{+}$,

- $n_{-}$particles $\mu_{p_{a}}^{(l)}$ and holes $\mu_{h_{a}}^{(l)}$ located at $-q$ in the thermodynamic limit: $\mu_{p_{a}}^{(l)}, \mu_{h_{a}}^{(l)} \underset{N, L \rightarrow+\infty}{\longrightarrow}-q$ for $a=1, \ldots, n_{-}$.

would give rise to the same (from the point of view of energy $\mathcal{E}=\varepsilon\left(\lambda_{0}\right)$, momentum $\mathcal{P}=p\left(\lambda_{0}\right)-p(q), \ldots$ ) macroscopic state. In a joint collaboration with Kitanine, Maillet, Slavnov and Terras we have shown [43] that indeed, in the zero-time case, the contribution of a given macroscopic state to the asymptotics is obtained by summing up over all such zero-momentum excitations on each of the Fermi boundaries. Clearly, this picture persists in the time-dependent case as well. The only difference being that, in the time-dependent case, the number of relevant macroscopic states contributing to the asymptotics is bigger (one has to include the contributions of excitations around the saddle-point in addition to the excitations on the Fermi boundary). Moreover, we would like to draw the reader's attention to the fact that it is precisely the sum over such zero momentum excitations on the Fermi boundary that gives rise, through some intricate microscopic mechanism of summation, to the relativistic combinations $\left(x+v_{F} t\right)^{\alpha_{+}}$(in what concerns the left Fermi boundary) and $\left(x-v_{F} t\right)^{\alpha_{-}}$(in what concerns the right Fermi boundary) arising in the asymptotics. This mechanism can be considered as yet another manifestation of conformal field theory on the level of asymptotics.

Our analysis leads us to propose an alternative interpretation of the universality hypothesis. Namely, when dealing with asymptotics (large-distance, etc) of correlation functions, one is brought to the analysis of the contributions of "relevant" saddle-points. As one can expect from the saddle-point type analysis of one-dimensional integrals, the leading asymptotics are only depending on the local behavior around the saddle-point of the driving term. All other details of the integrand do not matter for fixing the exponent governing the algebraic decay. Therefore, it is quite reasonable to expect that models sharing the same types of saddle-points exhibit the same type of critical behavior. The universality hypothesis [24] stating that models sharing the same symmetry class have the same value for their critical exponents can be now re-interpreted as the fact that the symmetries of a model uniquely determine the structure of the driving terms in the saddle-points that are relevant for the asymptotics. As a consequence, the leading power-law decay stemming from the local analysis around these saddle-points is always characterized by the same critical exponents regardless of the fine, model dependent, function content of the integrals describing the correlation functions.

We draw the reader's attention to the fact that the terms appearing in the $2^{\text {nd }}$ and $3^{\text {rd }}$ lines of (3.4) correspond solely to excitations on the Fermi boundaries and confirm the CFT/Luttinger liquid-based predictions for the long-distance asymptotic $f^{7}$ at $t=0$ due to the identifications following from (2.12):

$$
F_{\emptyset}^{\emptyset}(q)+1=\frac{Z^{-1}(q)}{2}, F_{\emptyset}^{\emptyset}(-q)=-\frac{Z^{-1}(q)}{2}, F_{q}^{-q}(q)=\frac{Z^{-1}(q)}{2}-Z(q), F_{q}^{-q}(-q)-1=-\frac{Z^{-1}(q)}{2}-Z(q) .
$$

\footnotetext{
The o (1) corrections being excluded

${ }^{\ddagger}$ Taking the $t \rightarrow 0$ limit of 3.4 is slightly subtle. The first line produces a contribution proportional to $t^{-\frac{1}{2}} \mathrm{e}^{i \frac{x^{2}}{4 t}}$. In the $t \rightarrow 0$ limit, this function approaches, in the sense of distributions, a Dirac $\delta(x)$ function. The presence of this $\delta(x)$ function is expected from the form of the commutation relations between the fields. However, in the large- $x$ limit of interest to us, it does not contribute.
} 
However, we do stress that (3.4) clearly shows the need to go beyond the CFT/Luttinger liquid picture so as to provide the correct long-time/large-distance asymptotic behavior of the correlation functions in gapless onedimensional quantum Hamiltonians. In particular, our results contain additional terms in respect to the predictions obtained in [2]. Our result support the predictions stemming from the non-linear Luttinger liquid theory for the behavior of the spectral function [12, 28].

\section{The form factor series}

In this section, we will provide two new representations for the zero-temperature reduced density matrix (3.1) starting from its form factor issued expansion:

$$
\rho_{N}(x, t)=\sum_{\substack{\ell_{1}<\cdots<\ell_{N+1} \\ \ell_{a} \in \mathbb{Z}}} \frac{\prod_{a=1}^{N+1} \mathrm{e}^{i x u_{0}\left(\mu_{\ell_{a}}\right)}}{\prod_{a=1}^{N} \mathrm{e}^{i x u_{0}\left(\lambda_{a}\right)}} \frac{\left.\left\langle\psi\left(\left\{\mu_{\ell_{a}}\right\}_{1}^{N+1}\right)\left|\Phi^{\dagger}(0,0)\right| \psi\left(\left\{\lambda_{a}\right\}_{1}^{N}\right)\right\rangle\right|^{2}}{\left\|\psi\left(\left\{\mu_{\ell_{a}}\right\}_{1}^{N+1}\right)\right\|^{2} \cdot\left\|\psi\left(\left\{\lambda_{a}\right\}_{1}^{N}\right)\right\|^{2}} .
$$

The above series runs through all the possible choices of integers $\ell_{a}, a=1, \ldots, N+1$ such that $\ell_{1}<\cdots<\ell_{N+1}$.

Below, we shall argue in favor of several reasonable approximations that allow us to reduce the form factor series to another, effective one, whose structure is simple enough so as to be able to continue the calculations directly on it.

\subsection{The effective form factors}

It has been shown in [64] (slightly different determinant representations for these form factors have already appeared in $[61,73]$ ) that the form factors of the operator $\Phi^{\dagger}$ taken between the $N$-particle ground state $\left\{\lambda_{a}\right\}_{1}^{N}$ and any particle-hole type excited state $\left\{\mu_{\ell_{a}}\right\}_{1}^{N+1}$ as described in (2.3) takes the form

$$
\frac{\left|\left\langle\psi\left(\left\{\mu_{\ell_{a}}\right\}_{1}^{N+1}\right)\left|\Phi^{\dagger}(0,0)\right| \psi\left(\left\{\lambda_{a}\right\}_{1}^{N}\right)\right\rangle\right|^{2}}{\left\|\psi\left(\left\{\mu_{\ell_{a}}\right\}_{1}^{N+1}\right)\right\|^{2}\left\|\psi\left(\left\{\lambda_{a}\right\}_{1}^{N}\right)\right\|^{2}}=\widehat{\mathcal{G}}_{N ; 1}\left(\begin{array}{l}
\left\{p_{a}\right\}_{1}^{n} \\
\left\{h_{a}\right\}_{1}^{n}
\end{array}\right)\left[\widehat{F}_{\left\{\ell_{a}\right\}}, \widehat{\xi}_{\left\{\ell_{a}\right\}}, \widehat{\xi}\right] \cdot \widehat{D}_{N}\left(\begin{array}{l}
\left\{p_{a}\right\}_{1}^{n} \\
\left\{h_{a}\right\}_{1}^{n}
\end{array}\right)\left[\widehat{F}_{\left\{\ell_{a}\right\}}, \widehat{\xi}_{\left\{\ell_{a}\right\}}, \widehat{\xi}\right]
$$

This representation involves two functionals, the so-called smooth part of the form factor $\widehat{\mathcal{G}}_{N ; 1}$ and the so-called discreet part $\widehat{D}_{N}$. These are functionals of the counting function $\widehat{\xi}$ for the ground state, of the counting function $\widehat{\xi}_{\left\{\ell_{a}\right\}}$ for the excited state and of the associated shift function $\widehat{F}_{\left\{\ell_{a}\right\}}$.

It has been show in [64], that, in the large $L$-limit and for any $n$ particle-hole type excited state, with $n$ bounded independently of $L$, these functionals satisfy

$$
\left(\widehat{\mathcal{G}}_{N ; 1} \widehat{D}_{N}\right)\left(\begin{array}{l}
\left\{p_{a}\right\}_{1}^{n} \\
\left\{h_{a}\right\}_{1}^{n}
\end{array}\right)\left[\widehat{F}_{\left\{\ell_{a}\right\}}, \widehat{\xi}_{\left\{\ell_{a}\right\}}, \widehat{\xi}\right]=\widehat{\mathcal{G}}_{N ; 1}\left(\begin{array}{c}
\left\{p_{a}\right\}_{1}^{n} \\
\left\{h_{a}\right\}_{1}^{n}
\end{array}\right)\left[F_{0}, \xi, \xi_{F_{0}}\right] \cdot \widehat{D}_{N}\left(\begin{array}{l}
\left\{p_{a}\right\}_{1}^{n} \\
\left\{h_{a}\right\}_{1}^{n}
\end{array}\right)\left[F_{0}, \xi, \xi_{F_{0}}\right]\left(1+\mathrm{O}\left(\frac{\ln L}{L}\right)\right)
$$

We stress that the functionals appearing on the rhs of the above equation act on

i) the thermodynamic limit $F_{0}(\lambda)$ of the shift function at $\beta=0$ associated to the excited state labeled by the set of integers $\left\{\ell_{a}\right\}_{1}^{N+1}(2.11)$,

ii) the thermodynamic limit $\xi(\lambda)$ of the counting function (2.6),

iii) the counting function associated with $F_{0}: \xi_{F_{0}}(\lambda)=\xi(\lambda)+F_{0}(\lambda) / L$. 
We do stress that the shift function $F_{0}$ depends implicitly on the rapidities of the particles $\left\{\mu_{p_{a}}\right\}_{1}^{n}$ and holes $\left\{\mu_{h_{a}}\right\}_{1}^{n}$ entering in the description of the excited state of interest, $c f(2.11)$. We chose not to write this dependence explicitly in (4.3) as the auxiliary arguments of $F_{0}$ are undercurrent by those of the functionals $\widehat{D}_{N}$ and $\widehat{G}_{N, 1}$. Given any holomorphic function $v(\lambda)$ in a neighborhood of $\mathbb{R}$, the explicit expressions for $\widehat{D}_{N}\left[v, \xi, \xi_{v}\right]$ (and $\widehat{G}_{N}\left[v, \xi, \xi_{v}\right]$ ) involves two sets of parameters $\left\{\lambda_{a}\right\}_{1}^{N}$ and $\left\{\mu_{\ell_{a}}\right\}_{1}^{N+1}$ which are defined as follows

- $\mu_{k}, k \in \mathbb{Z}$ is the unique* solution to $\xi\left(\mu_{k}\right)=k / L$, ie the second argument of the functionals;

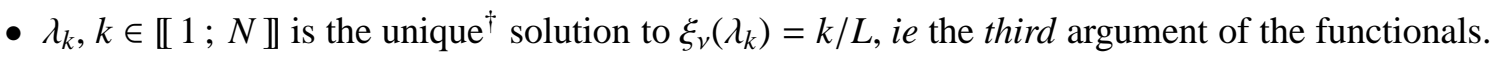

We insist that here and in the following, the parameters $\mu_{k}$ or $\lambda_{p}$ entering in the explicit expressions for these functionals are always to be understood in this way. Also, we remind that the integers $\ell_{a}$ are obtained from the integers $\left\{p_{a}\right\}_{1}^{n}$ and $\left\{h_{a}\right\}_{1}^{n}$ as explained in (2.3).

\section{- The discreet part}

The functional $\widehat{D}_{N}$ represents the universal part of the form-factor:

$$
\widehat{D}_{N}\left(\begin{array}{c}
\left\{p_{a}\right\}_{1}^{n} \\
\left\{h_{a}\right\}_{1}^{n}
\end{array}\right)\left[F_{0}, \xi, \xi F_{0}\right]=\frac{\prod_{k=1}^{N}\left\{4 \sin ^{2}\left[\pi F_{0}\left(\lambda_{k}\right)\right]\right\}}{\prod_{a=1}^{N+1} 2 \pi L \xi^{\prime}\left(\mu_{\ell_{a}}\right) \prod_{a=1}^{N} 2 \pi L \xi_{F_{0}}^{\prime}\left(\lambda_{a}\right)} \prod_{a=1}^{N}\left(\frac{\mu_{\ell_{a}}-\mu_{\ell_{N+1}}}{\lambda_{a}-\mu_{\ell_{N+1}}}\right)^{2} \operatorname{det}_{N}^{2}\left[\frac{1}{\mu_{\ell_{a}}-\lambda_{b}}\right] .
$$

The large $N, L$ behavior of (4.4) can be computed explicitly and is given in (A.2)-(A.4). However, it is the above finite product representation of $\widehat{D}_{N}$ that is suited for carrying out resummations.

\section{- The smooth part}

The functional $\widehat{\mathcal{G}}_{N, 1}$ represents the so-called smooth part of the form factor:

$$
\begin{aligned}
\widehat{\mathcal{G}}_{N ; \gamma}\left(\begin{array}{c}
\left\{p_{a}\right\} \\
\left\{h_{a}\right\}
\end{array}\right) & {\left[F_{0}, \xi, \xi_{F_{0}}\right]=\frac{V_{N ; 1}\left(\mu_{N+1}\right) V_{N ;-1}\left(\mu_{N+1}\right)}{\operatorname{det}_{N+1}\left[\Xi^{(\mu)}[\xi]\right] \operatorname{det}_{N}\left[\Xi^{(\lambda)}\left[\xi_{F_{0}}\right]\right]} W_{n}\left(\begin{array}{c}
\left\{\mu_{p_{a}}\right\}_{1}^{n} \\
\left\{\mu_{h_{a}}\right\}_{1}^{n}
\end{array}\right) \prod_{a=1}^{n} \prod_{\epsilon= \pm}\left\{\frac{V_{N ; \epsilon}\left(\mu_{p_{a}}\right)}{V_{N ; \epsilon}\left(\mu_{h_{a}}\right)} \frac{\mu_{h_{a}}-\mu_{N+1}+i \epsilon c}{\mu_{p_{a}}-\mu_{N+1}+i \epsilon c}\right\} } \\
& \times W_{N}\left(\begin{array}{c}
\left\{\lambda_{a}\right\}_{1}^{N} \\
\left\{\mu_{a}\right\}_{1}^{N}
\end{array}\right) \operatorname{det}_{N}\left[\delta_{j k}+\gamma \widehat{V}_{j k}\left[F_{0}\right]\left(\left\{\lambda_{a}\right\}_{1}^{N} ;\left\{\mu_{\ell_{a}}\right\}_{1}^{N+1}\right)\right] \operatorname{det}_{N}\left[\delta_{j k}+\gamma \overline{\bar{V}}_{j k}\left[F_{0}\right]\left(\left\{\lambda_{a}\right\}_{1}^{N} ;\left\{\mu_{\ell_{a}}\right\}_{1}^{N+1}\right)\right] .
\end{aligned}
$$

Above, we have introduced several functions. For any set of generic parameters $\left(\left\{z_{a}\right\}_{1}^{n} ;\left\{y_{a}\right\}_{1}^{n}\right) \in U_{\delta}^{n} \times U_{\delta}^{n}$

$$
W_{n}\left(\begin{array}{c}
\left\{z_{a}\right\}_{1}^{n} \\
\left\{y_{a}\right\}_{1}^{n}
\end{array}\right)=\prod_{a, b=1}^{n} \frac{\left(z_{a}-y_{b}-i c\right)\left(y_{a}-z_{b}-i c\right)}{\left(y_{a}-y_{b}-i c\right)\left(z_{a}-z_{b}-i c\right)} \quad \text { and } \quad V_{N ; \epsilon}(\omega)=\prod_{a=1}^{N} \frac{\omega-\lambda_{b}+i \epsilon c}{\omega-\mu_{b}+i \epsilon c}
$$

Also we have set

$$
\Xi_{j k}^{(\mu)}[\xi]=\delta_{j k}-\frac{K\left(\mu_{\ell_{a}}-\mu_{\ell_{b}}\right)}{2 \pi L \xi^{\prime}\left(\mu_{\ell_{b}}\right)} \quad \text { and } \quad \Xi_{j k}^{(\lambda)}\left[\xi_{F_{0}}\right]=\delta_{j k}-\frac{K\left(\lambda_{a}-\lambda_{b}\right)}{2 \pi L \xi_{F_{0}}^{\prime}\left(\lambda_{b}\right)}
$$

${ }^{*}$ The uniqueness follows from the fact that the dressed momentum $p(\lambda)$ is a biholomorphism on some sufficiently narrow strip $U_{\delta}$ around the real axis and that $p(\lambda) \in \mathbb{R} \Rightarrow \lambda \in \mathbb{R}$.

†The uniqueness follows from Rouché's theorem when L is large enough. 
Finally, for any set of generic parameters $\left(\left\{z_{a}\right\}_{1}^{n} ;\left\{y_{a}\right\}_{1}^{n+1}\right) \in U_{\delta}^{n} \times U_{\delta}^{n+1}$ the entries of the two determinants in the numerator read

$$
\begin{aligned}
\widehat{V}_{k \ell}[v]\left(\left\{z_{a}\right\}_{1}^{n} ;\left\{y_{a}\right\}_{1}^{n+1}\right)= & i \frac{\prod_{a=1}^{n+1}\left(z_{k}-y_{a}\right)}{\prod_{a \neq k}^{n}\left(z_{k}-z_{a}\right)} \frac{\prod_{a=1}^{n}\left(z_{k}-z_{a}+i c\right)}{\prod_{a=1}^{n+1}\left(z_{k}-y_{a}+i c\right)} \frac{K\left(z_{k}-z_{\ell}\right)}{\mathrm{e}^{-2 i \pi v\left(z_{k}\right)}-1} \\
\widehat{\bar{V}}_{k \ell}[v]\left(\left\{z_{a}\right\}_{1}^{n} ;\left\{y_{a}\right\}_{1}^{n+1}\right) & =-i \frac{\prod_{a=1}^{n+1}\left(z_{k}-y_{a}\right)}{\prod_{a \neq k}^{n}\left(z_{k}-z_{a}\right)} \frac{\prod_{a=1}^{n}\left(z_{k}-z_{a}-i c\right)}{\prod_{a=1}^{n+1}\left(z_{k}-y_{a}-i c\right)} \frac{K\left(z_{k}-z_{\ell}\right)}{\mathrm{e}^{2 i \pi v\left(z_{k}\right)}-1}
\end{aligned}
$$

Note that the singularities of the associated determinants at $z_{k}=z_{j}, j \neq k$ are only apparent, $c f$ [45, 64].

\subsection{Arguments for the effective form factors series}

It is believed that when computing the $T=0 K$ form factor expansion of a two-point function $\left\langle\right.$ G.S. $\left|O_{1} O_{2}\right|$ G.S. $\rangle$ on the intermediate excited states (as in (4.1)), the contribution of those excited states whose energies differ macroscopically from the ground state's one (ie by a quantity scaling as some positive power of $L$ ) vanishes in the $L \rightarrow+\infty$ limit. This can, for instance, be attributed to an extremely quick oscillation of the phase factors and the decay of form factors for states having large excitation momenta and energies. Therefore, we shall assume in the following that the only part of the form factor expansion in (4.1) that has a non-vanishing contribution to the thermodynamic limit $\rho(x, t)$ of $\rho_{N}(x, t)$ corresponds to a summation over all those excited states which are realized as some finite (in the sense that not scaling with $L$ ) number $n, n=0,1, \ldots$, of particle-hole excitations above the $(N+1)$-particle ground state. Indeed, these are the only excited states that can have a finite (ie not scaling with $L$ ) energy gap above the ground state in the $N$-particle sector.

Even when dealing with excited states realized as a finite number $n$ of particle-hole excitations above the $(N+1)$-particle ground state, it is still possible to generate a macroscopically different energy from the one of the $N$-particle ground state if the rapidities of the particles become very large (ie scale with $L$ ). This case corresponds, among others, to integers $p_{a}$ becoming very large and scaling with $L$. We will drop the contribution of such excited states in the following.

Limiting the sum over all the excited states in the $(N+1)$-particle sector to those having the same per-site energy that the ground state means that one effectively neglects correcting terms in the lattice size $L$. It thus seems very reasonable to assume that, on the same ground, only the leading large- $L$ asymptotic behavior of the form factors will contribute to the thermodynamic limit of $\rho_{N}(x, t)$. It is clearly so when focusing on states with a low number $n$ of particle/hole excitations. However, in principle, problems could arise when the number $n$ becomes of the order of $L$. Our assumption lead to the following consequences:

- we discard all summations over the excited states having a too large excitation energy. This means that we introduce a "cut-off" in respect to the range of the integers entering in the description of the rapidities of the particles. Namely, we assume that the integers $p_{a}$ are restricted to belong to the set:

$$
\mathcal{B}_{L}^{\mathrm{ext}} \equiv\left\{n \in \mathbb{Z}:-w_{L}<n<w_{L}\right\} \backslash \llbracket 1 ; N+1 \rrbracket \quad \text { where } \quad w_{L} \sim L^{1+\frac{1}{4}} .
$$

\footnotetext{
${ }^{\dagger}$ The computations presented in appendices B.2 and B.3 can be seen as a proof of this statement in the case of a generalized free fermion model.

${ }^{\ddagger}$ Note that we could choose $w_{L}$ to scale as $L^{1+\epsilon}$, where $\epsilon>0$ is small enough but arbitrary otherwise. We choose $\epsilon=1 / 4$ for definiteness. $c f$ appendix B.1 for a better discussion of the origin of such a property.
} 
- The oscillating exponent $\sum_{a=1}^{N+1} u_{0}\left(\mu_{\ell_{a}}\right)-\sum_{a=1}^{N} u_{0}\left(\lambda_{a}\right)$ is replaced by its thermodynamic limit as given in (2.13).

- We drop the contribution of the $\mathrm{O}\left(L^{-1} \cdot \ln L\right)$ terms in the large-size behavior of form factors given in (4.3).

Note that, within our approximations, the localization of the Bethe roots $\left\{\mu_{\ell_{a}}\right\}_{1}^{N+1}$ for an excited state whose particles' (resp. holes') rapidities are labeled by the integers $\left\{p_{a}\right\}_{a=1}^{n}$ (resp. $\left\{h_{a}\right\}_{a=1}^{n}$ ) does not depend on the specific choice of the excited state one considers. Hence, we effectively recover a description of the excitations that is in the spirit of a free fermionic model.

Our simplifying hypothesis suggest to raise the below conjecture

Conjecture 4.1 The thermodynamic limit of the reduced density matrix $\rho_{N}(x, t)$ coincides with the thermodynamic limit of the effective reduced density matrix $\rho_{N ; \text { eff }}(x, t)$ :

$$
\lim _{N, L \rightarrow+\infty} \rho_{N}(x, t)=\lim _{N, L \rightarrow+\infty} \rho_{N ; \text { eff }}(x, t)
$$

where $\rho_{N ; \text { eff }}(x, t)$ is given by the series

$$
\rho_{N ; \text { eff }}(x, t)=\sum_{n=0}^{N+1} \sum_{\substack{p_{1}<\cdots<p_{n} \\
p_{a} \in \mathcal{B}_{L}^{\text {ext }}}} \sum_{h_{1}<\cdots<h_{n}} \prod_{\substack{a=1 \\
h_{a} \in \mathcal{B}_{L}^{\text {int }}}}^{n} \frac{\mathrm{e}^{-i x u\left(\mu_{h_{a}}\right)}}{\mathrm{e}^{-i x u\left(\mu_{p_{a}}\right)}} \cdot\left(\widehat{D}_{N, n} \widehat{\mathcal{G}}_{N ; 1}\right)\left(\begin{array}{c}
\left\{p_{a}\right\} \\
\left\{h_{a}\right\}
\end{array}\right)\left[F_{0}\left(* \mid \begin{array}{c}
\left\{\mu_{p_{a}}\right\} \\
\left\{\mu_{h_{a}}\right\}
\end{array}\right) ; \xi ; \xi_{F_{0}}\right] .
$$

There $\mathcal{B}_{L}=\left\{n \in \mathbb{Z}:-w_{L}<n<w_{L}\right\}, \quad \mathcal{B}_{L}^{\text {ext }}=\mathcal{B}_{L} \backslash \llbracket 1 ; N+1 \rrbracket$ and $\mathcal{B}_{L}^{\text {int }}=\llbracket 1 ; N+1 \rrbracket$. Also, the $*$ refers to the running variable of $F_{0}$ on which the two functionals act.

The effective form factor series (4.11) possesses several different features in respect to the form factor expansionbased series that would appear in a generalized free fermion model $(c f(\overline{B .20})$. Namely,

- the shift function $F_{0}$ depends parametrically on the rapidities of the particles and holes entering in the description of each excited state one considers, $c f$ (2.11). It is thus summation dependent.

- Each summand is weighted by the factor $\widehat{\mathcal{G}}_{N ; 1}$ that takes into account the more complex structure of the scattering and of the scalar products in the interacting model. This introduces a strong coupling between the summation variables $\left\{p_{a}\right\}_{1}^{n}$ and $\left\{h_{a}\right\}_{1}^{n}$. Indeed, the explicit expression for $\widehat{\mathcal{G}}_{N ; 1}$ involves complicated functions of the rapidities $\left\{\mu_{p_{a}}\right\}_{1}^{n}$ and $\left\{\mu_{h_{a}}\right\}_{1}^{n}$.

A separation of variables that would allow one for a resummation of (4.11) is not possible for precisely these two reasons. To overcome this problem we proceed in several steps. First, we introduce a $\gamma$-deformation of the effective form factor series such that $\rho_{N \text {;eff }}(x, t \mid \gamma)_{\mid \gamma=1}=\rho_{N \text {;eff }}(x, t)$ :

$$
\rho_{N ; \mathrm{eff}}(x, t \mid \gamma)=\sum_{n=0}^{N+1} \sum_{\substack{p_{1}<\cdots<p_{n} \\
p_{a} \in \mathcal{B}_{L}^{\text {ext }}}} \sum_{h_{1}<\cdots<h_{n}} \prod_{\substack{h_{a} \in \mathcal{B}_{L}^{\text {int }} \\
h^{\prime}}}^{n} \frac{\mathrm{e}^{-i x u\left(\mu_{h_{a}}\right)}}{\mathrm{e}^{-i x u\left(\mu_{p a}\right)}}\left(\widehat{D}_{N, n} \widehat{\mathcal{G}}_{N ; \gamma}\right)\left(\begin{array}{l}
\left\{p_{a}\right\} \\
\left\{h_{a}\right\}
\end{array}\right)\left[\gamma F_{0}\left(* \mid \begin{array}{c}
\left\{\mu_{p_{a}}\right\} \\
\left\{\mu_{h_{a}}\right\}
\end{array}\right) ; \xi ; \xi_{\gamma F_{0}}\right] .
$$

For any finite $N$ and $L$, it is readily checked by using the explicit representations (4.4) for $\widehat{D}_{N, n}$ and (4.5) for $\widehat{\mathcal{G}}_{N ; \gamma}$ that the $\gamma$-deformation $\rho_{N \text {;eff }}(x, t \mid \gamma)$ is holomorphic in $\gamma$ belonging to an open neighborhood of the closed unit 
disct. Hence, its Taylor series around $\gamma=0$ converges up to $\gamma=1$. We will then show in theorem C.1 that, given any fixed $m$, the $m^{\text {th }}$ Taylor coefficient of $\rho_{N \text {;eff }}(x, t \mid \gamma)$ at $\gamma=0$ :

$$
\rho_{N ; \mathrm{eff}}^{(m)}(x, t)=\left.\frac{\partial^{m}}{\partial \gamma^{m}} \rho_{N ; \mathrm{eff}}(x, t \mid \gamma)\right|_{\gamma=0},
$$

can be re-summed into a representation where the existence of the thermodynamic limit $\rho_{\mathrm{eff}}^{(m)}(x, t)$ is readily seen. This fact is absolutely not-clear on the level of (4.13) as, due to (A.3)-(A.4), each individual summand vanishes as a complicated power-law in $\mathrm{L}$ that depends on the excited state considered. We will then show that one can represent the thermodynamic limit $\rho_{\mathrm{eff}}^{(m)}(x, t)$ in another way. This representation is given in terms of a finite sum of multiple integrals and corresponds to a truncation of the so-called multidimensional Natte series that we introduce below. The latter description of $\rho_{\mathrm{eff}}^{(m)}(x, t)$ gives a straightforward access to its asymptotic expansion.

The proof of the existence of the thermodynamic limit and the construction of the truncated multidimensional Natte series for $\rho_{\mathrm{eff}}^{(m)}(x, t)$ constitute the rigorous and conjecture free part of our analysis. This is summarized in theorem 4.1 .

Working on the Taylor coefficients $\rho_{N \text {;eff }}^{(m)}(x, t)$ instead of the full function $\rho_{N \text {;eff }}(x, t \mid \gamma)$ taken at $\gamma=1$ has the advantage of separating all questions of convergence of the representations we obtain from the question of well-definiteness of the various re-summations and deformation procedures that we carry out on $\rho_{N ; \text { eff }}^{(m)}(x, t)$ (and subsequently on $\rho_{\text {eff }}^{(m)}(x, t)$ once that the thermodynamic limit is taken). Indeed, by taking the $m^{\text {th }} \gamma$-derivative at $\gamma=0$, we always end up dealing with a finite number of sums. However, if we had carried out the forthcoming re-summation directly on the level of $\rho_{\text {eff }}(x, t)$, we would have ended up with a series of multiple integrals instead of a finite sum. The convergence of such a series constitutes a separate question that deserves, in its own right, another study. Nonetheless, in the present paper, in order to provide physically interesting results, we will take this convergence as a reasonable conjecture in a subsequent part of the paper.

\subsection{An operator ordering}

Prior to carrying out the re-summation of the form factor expansion for $\rho_{N \text {;eff }}^{(m)}(x, t)$, we need to discuss a way of representing functional translations and generalizations thereof. These objects will allow us to separate the variables in the sum (4.13), and carry out the various re-summations. A more precise analysis and discussion of these constructions is postponed to appendix $\mathrm{D}$ In the following, we denote by $\mathscr{O}(W)$, the ring of holomorphic functions in $\ell$ variables on $W \subset \mathbb{C}^{\ell}$. Also, here and in the following $f \in \mathscr{O}(W)$, with $W$ non-open means that $f$ is a holomorphic function on some open neighborhood of $W$. Finally, for a set $S$ on which the function $f$ is defined we denote $\|f\|_{S}=\sup _{s \in S}|f(s)|$.

Throughout this paper we will deal with various examples $\left(\widehat{D}_{N}, \widehat{\mathcal{G}}_{N}^{(\beta)}, \ldots\right)$ of functionals $\mathcal{F}[v]$ acting on holomorphic functions $v$. The function $v$ will always be defined on some compact subset $M$ of $\mathbb{C}$ whereas the explicit expression for $\mathcal{F}[v]$ will only involve the values taken by $v$ on a smaller compact $K \subset \operatorname{Int}(M)$. In fact, all the functionals that we will consider share the regularity property below:

Definition 4.1 Let $M, K$ be compacts in $\mathbb{C}$ such that $K \subset \operatorname{Int}(M)$. Let $W_{z}$ be a compact in $\mathbb{C}^{\ell_{z}}, \ell_{z} \in \mathbb{N} \equiv\{0,1, \ldots\}$. An $\ell_{z}$-parameter family of functionals $\mathcal{F}[\cdot](z)$ depending on a set of auxiliary variables $z \in W_{z}$ is said to be regular (in respect to the pair $(M, K)$ ) if

i) there exists constants $C_{\mathcal{F}}>0$ and $C^{\prime}>0$ such that for any $f, g \in \mathscr{O}(M)$

$$
\|f\|_{K}+\|g\|_{K}<C_{\mathcal{F}} \Rightarrow\|\mathcal{F}[f](\cdot)-\mathcal{F}[g](\cdot)\|_{W_{z}}<C^{\prime}\|f-g\|_{K},
$$

The apperent singularity of the determinants at $\mathrm{e}^{ \pm 2} i \pi F_{0}\left(\lambda_{k}\right)-1=0, c f\left[4.8\right.$, are candelled by the pre-factors $\sin ^{2}\left[\pi F_{0}\left(\lambda_{k}\right)\right]$ present in $\widehat{D}_{N}, c f$ (4.4). 
where the $\cdot$ indicates that the norm is computed in respect to the set of auxiliary variables $z \in W_{z}$.

ii) Given any open neighborhood $W_{y}$ of 0 in $\mathbb{C}^{\ell_{y}}$, for some $\ell_{y} \in \mathbb{N}$, if $v(\lambda, \boldsymbol{y}) \in \mathscr{O}\left(M \times W_{y}\right)$ is such that $\|v\|_{K \times W_{y}}<$ $C_{\mathcal{F}}$, then the function $(\boldsymbol{y}, \boldsymbol{z}) \mapsto \mathcal{F}[v(*, \boldsymbol{y})](z)$ is holomorphic on $W_{y} \times W_{z}$. Here, the $*$ indicated the running variable $\lambda$ of $v(\lambda, \boldsymbol{y})$ on which the functional $\mathcal{F}[\cdot](z)$ acts.

The constant $C_{\mathcal{F}}$ appearing above will be called constant of regularity of the functional.

This regularity property is at the heart of the aforementioned representation for the functional translation and generalizations thereof that we briefly discuss below. However, prior to this discussion we need to define the discretization of the boundary of a compact.

Definition 4.2 Let $M$ be a compact with $n$ holes (ie $\mathbb{C} \backslash M$ has $n$ bounded connected components) and such that $\partial M$ can be realized as a disjoint union of $n+1$ smooth Jordan curves $\gamma_{a}:[0 ; 1] \rightarrow \partial M$, ie $\partial M=\bigsqcup_{a=1}^{n+1} \gamma_{a}([0 ; 1])$. $A$ discretization (of order $s$ ) of $\partial M$ will correspond to a collection of $(n+1)(s+2)$ points $t_{j, a}=\gamma_{a}\left(x_{j}\right)$ with $j=0, \ldots, s+1$ and $a=1, \ldots, n+1$ where $x_{0}=0 \leq x_{1}<\cdots<x_{s} \leq 1=x_{s+1}$ is a partition of $[0 ; 1]$ of mesh $2 / s:\left|x_{j+1}-x_{j}\right| \leq 2 / s$.

\subsubsection{Translations}

Suppose that one is given a compact $M$ in $\mathbb{C}$ without holes whose boundary is a smooth Jordan curve $\gamma:[0 ; 1] \rightarrow$ $\partial M$. Let $K$ be a compact such that $K \subset \operatorname{Int}(M)$ and $\mathcal{F}$ a regular functional ( $c f$ definition 4.1 ) in respect to $(M, K)$, for simplicity, not depending on auxiliary parameters $z$.

It is shown in proposition D.1 that, then, for $|\gamma|$ small enough one has the identity

$$
\mathcal{F}\left[\gamma W_{n}\left(* \mid \begin{array}{l}
\left\{y_{a}\right\}_{1}^{n} \\
\left\{z_{a}\right\}_{1}^{n}
\end{array}\right)\right]=\lim _{s \rightarrow+\infty}\left\{\prod_{a=1}^{n} \mathrm{e}^{\widehat{\widehat{g}}_{s}\left(y_{a}\right)-\widehat{g}_{s}\left(z_{a}\right)} \cdot \mathcal{F}\left[\gamma f_{s}\right]\right\}_{\mid s_{k}=0} .
$$

The function $W_{n}$ appearing above is defined in terms of an auxiliary function $\psi(\lambda, \mu)$ that is holomorphic on $M \times M$

$$
W_{n}\left(\lambda \mid \begin{array}{l}
\left\{y_{a}\right\}_{1}^{n} \\
\left\{z_{a}\right\}_{1}^{n}
\end{array}\right)=\sum_{a=1}^{n} \psi\left(\lambda, y_{a}\right)-\psi\left(\lambda, z_{a}\right) \quad \text { whereas } \quad f_{s}\left(\lambda \mid\left\{\varsigma_{a}\right\}_{1}^{s}\right) \equiv f_{s}(\lambda)=\sum_{j=1}^{s} \frac{\left(t_{j+1}-t_{j}\right)}{t_{j}-\lambda} \cdot \frac{\varsigma_{j}}{2 i \pi} .
$$

Finally, $\widehat{g}_{s}(\lambda)$ is a differential operator in respect to $\varsigma_{a}$, with $a=1, \ldots, s$ :

$$
\widehat{g}_{s}(\lambda)=\sum_{j=1}^{s} \psi\left(t_{j}, \lambda\right) \frac{\partial}{\partial \varsigma_{j}}
$$

The definitions of $\widehat{g}_{s}$ and $f_{s}$ involve a set of $s+1$ discretization points $t_{j}$ of $\partial M$.

The limit in (4.15) is uniform in the parameters $y_{a}$ and $z_{a}$ belonging to $M$ and in $|\gamma|$ small enough. Actually, the magnitude of $\gamma$ depends on the value of the constant of regularity $C_{\mathcal{F}}$. If the latter is large enough, one can even set $\gamma=1$. The limit in (4.15) also holds uniformly in respect to any finite order partial derivative of the auxiliary parameters. In particular,

$$
\prod_{a=1}^{n}\left\{\frac{\partial^{p_{a}}}{\partial y_{a}^{p_{a}}} \frac{\partial^{h_{a}}}{\partial z_{a}^{h_{a}}}\right\} \cdot \frac{\partial^{m}}{\partial \gamma^{m}} \mathcal{F}\left[\gamma W_{n}\left(* \mid \begin{array}{c}
\left\{\mu_{p_{a}}\right\} \\
\left\{\mu_{h_{a}}\right\}
\end{array}\right)\right]_{\mid \gamma=0}=\lim _{s \rightarrow+\infty}\left\{\prod_{a=1}^{n}\left\{\frac{\partial^{p_{a}}}{\partial y_{a}^{p_{a}}} \frac{\partial^{h_{a}}}{\partial z_{a}^{h_{a}}}\right\} \prod_{a=1}^{n} \mathrm{e}^{\widehat{g}_{s}\left(y_{a}\right)-\widehat{g}_{s}\left(z_{a}\right)} \cdot \frac{\partial^{m}}{\partial \gamma^{m}} \mathcal{F}\left[\gamma f_{s}\right]\right\}_{\substack{s_{k}=0 \\
\gamma=0}} .
$$



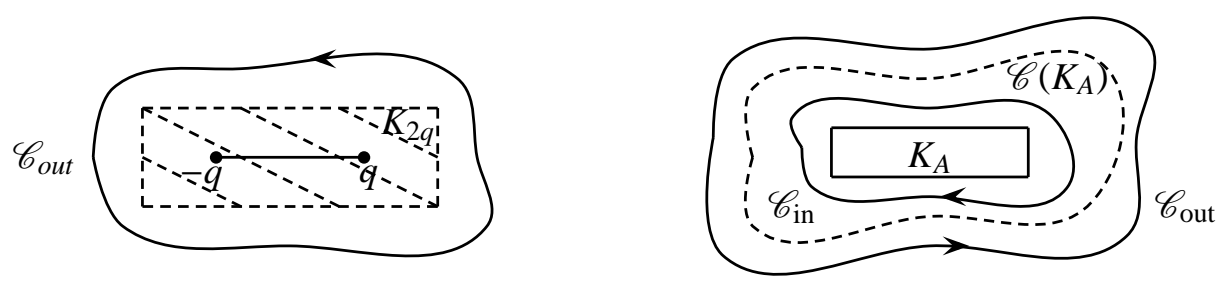

Figure 1: Example of discretized contours. In the $l h s$ the compact $M$ is located inside of its boundary $\mathscr{C}_{\text {out }}$ whereas the compact $K$ corresponds to $K_{2 q}$ as defined in (4.27). In this case $M$ has no holes. In the rhs the compact $M$ is delimited by the two Jordan curves $\mathscr{C}_{\text {in }}$ and $\mathscr{C}_{\text {out }}$ depicted in solid lines. The associated compact $K$ (of definition 4.1) corresponds to the loop $\mathscr{C}\left(K_{A}\right)$ depicted by dotted lines. The compact $M$ depicted in the rhs has one hole. This hole contains a compact $K_{A}$ inside.

We refer to appendix $\mathrm{D}$ for a proof of the above statement. Here, we would like to describe in words how formula (4.15) works. By properly tuning the value of $\gamma$ and invoking the regularity property of the functional $\mathcal{F}\left[\gamma f_{s}\right]$ one gets that, for any $s,\left\{\varsigma_{a}\right\}_{1}^{s} \mapsto \mathcal{F}\left[\gamma f_{s}\right]$ is holomorphic in a sufficiently large neighborhood of $0 \in \mathbb{C}^{s}$. This allows one to act with the translation operators $\prod_{b=1}^{n} \mathrm{e}^{\widehat{g}_{s}\left(y_{b}\right)-\widehat{g}_{s}\left(z_{b}\right)}$. Their action replaces each variable $\varsigma_{a}$ occurring in $f_{s}$ by the combination $\sum_{b=1}^{n}\left[\psi\left(t_{a}, y_{b}\right)-\psi\left(t_{a}, z_{b}\right)\right]$. Taking the limit $s \rightarrow+\infty$ changes the sum over $t_{a}$ occurring in $f_{s}$ into a contour integral over $\mathscr{C}_{\text {out }}, c f l h s$ of Fig. 1. Due to the presence of a pole at $t=\lambda$, this contour integral exactly reproduces the function $W_{n}$ that appears in the $r h s$ of (4.15).

Note that such a realization of the functional translation can also be build in the case of compacts $M$ having several holes as depicted in the $r h s$ of Fig. 1. Also, there is no problem to consider regular functionals $\mathcal{F}[\cdot](z)$ that depend on auxiliary sets of parameters $z$.

\subsubsection{Generalization of translations}

In the course of our analysis, in addition to dealing with functional translations as defined above, we will also have to manipulate more involved expressions involving series of partial derivatives. Namely, assume that one is given a regular functional $\mathcal{F}[f, g]$ of two arguments $f$ and $g$. Then, the expression : $\partial_{\gamma}^{m} \mathcal{F}\left[\gamma f_{s}, \widehat{g}_{s}\right]_{\mid \gamma=0}:$ is to be understood as the left substitution of the various $\partial_{S_{a}}$ derivatives symbols stemming from $\widehat{g}_{s}$.

More precisely, let $\widetilde{g}_{s}$ be the below holomorphic function of $a_{1}, \ldots, a_{s}$

$$
\widetilde{g}_{s}(\lambda)=\sum_{j=1}^{s} \psi\left(t_{j}, \lambda\right) a_{j}
$$

The regularity of the functional $\mathcal{F}$ ensures that the function $\left\{a_{p}\right\} \mapsto \partial_{\gamma}^{m} \mathcal{F}\left[\gamma f_{s}, \widetilde{g}_{s}\right]$ is holomorphic in $a_{1}, \ldots, a_{s}$ small enough. As a consequence, the below multi-dimensional series is convergent for $a_{j}$ small enough:

$$
\frac{\partial^{m}}{\partial \gamma^{m}} \cdot \mathcal{F}\left[\gamma f_{s}, \widetilde{g}_{s}\right]_{\mid \gamma=0}=\sum_{n_{j} \geq 0} \prod_{j=1}^{s}\left\{\frac{a_{j}^{n_{j}}}{n_{j} !} \frac{\partial^{n_{j}}}{\partial a_{j}^{n_{j}}}\right\} \frac{\partial^{m}}{\partial \gamma^{m}} \cdot \mathcal{F}\left[\gamma f_{s}, \widetilde{g}_{s}\right]_{\mid \begin{array}{l}
\gamma=0 \\
a_{j}=0
\end{array}} .
$$


We stress that as $f_{s}$ (4.16) is a holomorphic function of $\varsigma_{1}, \ldots, \varsigma_{s}$, the functional of $f_{s}$ coefficients of the above series give rise to a family of holomorphic functions in the variables $\varsigma_{1}, \ldots, \varsigma_{s}$. This analyticity follows, again, from the regularity of the functional $\mathcal{F}[f, g]$ and the smallness of $|\gamma|$.

The : $:$ ordering constitutes in substituting $a_{j} \hookrightarrow \partial_{\varsigma_{j}}, j=1, \ldots, s$ in such a way that all differential operators appear to the left. That is to say,

$$
: \frac{\partial^{m}}{\partial \gamma^{m}} \cdot \mathcal{F}\left[\gamma f_{s}, \widehat{g}_{s}\right]_{\mid \gamma=0}: \equiv \sum_{n_{j} \geq 0} \prod_{j=1}^{s}\left\{\frac{\partial^{n_{j}}}{\partial \varsigma_{j}^{n_{j}}}\right\} \cdot \prod_{j=1}^{s}\left\{\frac{1}{n_{j} !} \frac{\partial^{n_{j}}}{\partial a_{j}^{n_{j}}}\right\} \cdot \frac{\partial^{m}}{\partial \gamma^{m}} \cdot \mathcal{F}\left[\gamma f_{s}, \widetilde{g}_{s}\right]_{\left.\right|_{a_{j}=0 ; \varsigma_{j}=0}} .
$$

Although there where no convergence issues on the level of expansion (4.20), these can a priori arise on the level of the rhs in 4.21. Clearly, convergence depends on the precise form of the functional $\mathcal{F}$, and should thus be studied on a case-by-case basis. However, in the case of interest to us, this will not be a problem due to the quite specific class of functionals that we will deal with.

At this point, two observations are in order.

- (4.21) bears a strong resemblance with an $s$-dimensional Lagrange series.

- The functional (of $f_{s}$ ) coefficients appearing in the $r h s$ of (4.21) are completely determined by the functional $\mathcal{F}\left[\gamma f_{s}, \widetilde{g}_{s}\right]$ whose expression only involves standard (ie non-operator valued) functions. Should this functional have two (or more) equivalent representations, then any one of them can be used as a starting point for computing the coefficients in (4.20) and then carrying out the substitution (4.21).

Actually, for the class of functionals that we focus on, no convergence issues arise. Indeed, in all of the cases, the $m^{\text {th }} \gamma$-derivative at $\gamma=0$ of the : · : ordered functionals of interest appears as a finite linear combinations (or integrals thereof) of expressions of the type

$$
\widehat{\mathscr{E}}_{m}=: \frac{\partial^{m}}{\partial \gamma^{m}}\left\{\prod_{a=1}^{r} \mathrm{e}^{\epsilon_{a} \widehat{g}_{s}\left(\lambda_{\alpha_{a}}\right)} \cdot \prod_{b=1}^{\widetilde{r}} \mathrm{e}^{v_{b} \widehat{g}_{s}\left(y_{b}\right)} \cdot \mathcal{F}\left[\gamma f_{s}\right]\right\}_{\mid \gamma=0}: \quad \text { where } \alpha_{a} \in \mathbb{L} 1 ; N \rrbracket \quad \text { and } \quad \epsilon_{a}, v_{b} \in\{ \pm 1\} .
$$

Above $y_{a}$ are some auxiliary and generic parameters whereas $\lambda_{\alpha_{a}}$ are implicit functions of $\gamma$ and $\varsigma_{1}, \ldots, \varsigma_{s}$. For $L$-large enough, $\lambda_{\alpha_{a}}$ is the unique solution to the equation $\xi_{\gamma f_{s}}\left(\lambda_{\alpha_{a}}\right)=\alpha_{a} / L$.

The prescription that we have agreed upon implies that one should first substitute $\widehat{g}_{s} \hookrightarrow \widetilde{g}_{s}$ as defined in (4.19). Then, one computes the $m^{\text {th }} \gamma$-derivative at $\gamma=0$ of (4.22), this in the presence of non-operator valued functions $\widetilde{g}_{s}$. In the process, one has to differentiate in respect to $\gamma$ the functional $\mathcal{F}\left[\gamma f_{s}\right]$ and the arguments of $\widetilde{g}_{s}\left(\lambda_{\alpha_{a}}\right)$. Using that $\lambda_{\alpha_{a} \mid \gamma=0}=\mu_{\alpha_{a}}$, one arrives to

$$
\widetilde{\mathscr{E}}_{m} \equiv \frac{\partial^{m}}{\partial \gamma^{m}}\left\{\prod_{b=1}^{r} \mathrm{e}^{\epsilon_{b} \widetilde{g}_{s}\left(\lambda_{\alpha_{b}}\right)} \prod_{b=1}^{\widetilde{r}} \mathrm{e}^{v_{b} \widetilde{g}_{s}\left(y_{b}\right)} \cdot \mathcal{F}\left[\gamma f_{s}\right]\right\}_{\mid \gamma=0}=\prod_{b=1}^{r} \mathrm{e}^{\epsilon_{b} \widetilde{g}_{s}\left(\mu_{\alpha_{b}}\right)} \prod_{b=1}^{\widetilde{r}} \mathrm{e}^{v_{b} \widetilde{g}_{s}\left(y_{b}\right)} \sum_{n_{1}, \ldots, n_{s}=0}^{m} \prod_{j=1}^{s} a_{j}^{n_{j}} \cdot c_{\left\{n_{j}\right\}}\left[f_{s}\right] .
$$

The sum is truncated at most at $n_{j}=m, j=1, \ldots, m$ due to taking the $m^{\text {th }} \gamma$-derivative at $\gamma=0$. It is readily verified that the $\left\{n_{j}\right\}$-dependent coefficients $c_{\left\{n_{j}\right\}}\left[f_{s}\right]$ are regular functionals of $f_{s}$ with sufficiently large constants of regularity. It remains to impose the operator substitution on the level of (4.23) $a_{j} \hookrightarrow \partial_{S_{j}}$ with all differential operators $\partial_{\zeta k}, k=1, \ldots, s$ appearing to the left. It is clearly not a problem to impose such an operator order on the level of the polynomial part of the above expression. Indeed, the regularity of the functionals $c_{\left\{n_{j}\right\}}\left[f_{s}\right]$ implies that these are holomorphic in $\varsigma_{1}, \ldots, \varsigma_{s}$ belonging to an open neighborhood $\mathcal{N}_{0}$ of $0 \in \mathbb{C}^{s}$. Hence, $\prod_{k=1}^{s} \partial_{S_{k}}^{m_{k}} \cdot c_{\left\{n_{j}\right\}}\left[f_{s}\right]_{\mathcal{S}_{k}=0}$ is well-defined for any set of integers $\left\{m_{k}\right\}$. In fact, in all the cases of interest for us, the neighborhood $\mathcal{N}_{0}$ is always large enough so as to make the Taylor series issued from the products of translation 
operators $\prod_{a=1}^{r} \mathrm{e}^{\epsilon_{a} \widehat{g}_{s}\left(\mu_{\alpha_{a}}\right)} \prod_{b=1}^{\widetilde{r}} \mathrm{e}^{v_{b} \widehat{g}_{s}\left(y_{b}\right)}$ convergent. Their action can then be incorporated by a re-definition of $f_{s}$ leading to

$$
\mathscr{E}_{m}=\sum_{n_{1}, \ldots, n_{s}=0}^{m} \prod_{j=1}^{s}\left\{\frac{1}{n_{j} !} \frac{\partial^{n_{j}}}{\partial \varsigma_{j}^{n_{j}}}\right\} \cdot c_{\left\{n_{j}\right\}}\left[\widetilde{f}_{s}\right]_{\mid S_{k}=0}
$$

with

$$
\widetilde{f}_{s}(\lambda)=f_{s}(\lambda)+\sum_{b=1}^{s} \frac{\left(t_{b+1}-t_{b}\right)}{2 i \pi\left(t_{b}-\lambda\right)}\left\{\sum_{k=1}^{r} \epsilon_{k} \psi\left(t_{b}, \mu_{\alpha_{k}}\right)+\sum_{k=1}^{\widetilde{r}} v_{k} \psi\left(t_{b}, y_{k}\right)\right\} .
$$

In this way, one obtains a (truncated to a finite number of terms) s-dimensional Lagrange series. The procedure for dealing with such series and taking their $s \rightarrow+\infty$ limits is described in proposition D.2. In the following, all operator valued expressions ordered by $: \cdot$ : should be understood in this way.

\subsection{Resummation of the finite-volume Taylor coefficients}

In order to carry out the re-summation of the effective form factor expansion with the help of functional translations and generalizations thereof, we need to regularize the expression for the functional $\widehat{\mathcal{G}}_{N ; \gamma}$ with the help of an additional parameter $\beta$. This regularization will allow us to represent it as a regular functional that, moreover, has a form suitable for carrying out the intermediate calculations.

\section{The parameter $\beta$}

It is easy to see that

$$
\left(\widehat{D}_{N} \widehat{\mathcal{G}}_{N ; \gamma}\right)\left(\begin{array}{l}
\left\{p_{a}\right\}_{1}^{n} \\
\left\{h_{a}\right\}_{1}^{n}
\end{array}\right)\left[\gamma F_{0} ; \xi ; \xi_{\gamma F_{0}}\right]=\lim _{\beta \rightarrow 0}\left\{\widehat{D}_{N}\left(\begin{array}{l}
\left\{p_{a}\right\}_{1}^{n} \\
\left\{h_{a}\right\}_{1}^{n}
\end{array}\right)\left[\gamma F_{\beta} ; \xi ; \xi_{\gamma F_{\beta}}\right] \widehat{\mathcal{G}}_{N ; \gamma}\left(\begin{array}{l}
\left\{p_{a}\right\}_{1}^{n} \\
\left\{h_{a}\right\}_{1}^{n}
\end{array}\right)\left[\gamma F_{\beta} ; \xi ; \xi_{\gamma F_{\beta}}\right]\right\}
$$

We now introduce a prescription for taking the $\beta \rightarrow 0$ limit. When considered as a separate object from $\widehat{D}_{N}$, the functional $\widehat{\mathcal{G}}_{N ; \gamma}$ may exhibit singularities should it happen that $\gamma^{-1}\left\{\mathrm{e}^{2 i \pi \gamma F_{\beta}\left(\lambda_{j}\right)}-1\right\}=0$, cf (4.5)-(4.8). For $|\gamma|$ small enough, as it will always be the case for us, such potential zeroes correspond to the existence of solutions to $F_{\beta}\left(\lambda_{j}\right)=0$. For $\beta \in \widetilde{\boldsymbol{U}}_{\beta_{0}}$ with

$$
\widetilde{\boldsymbol{U}}_{\beta_{0}}=\left\{z \in \mathbb{C}: 10 \mathfrak{R}\left(\beta_{0}\right) \geq \mathfrak{R}(z) \geq \mathfrak{R}\left(\beta_{0}\right) \text { and }|\mathfrak{J}(z)| \leq \mathfrak{J}\left(\beta_{0}\right)\right\}
$$

$\mathfrak{R}\left(\beta_{0}\right)>0$ large enough and $\mathfrak{J}\left(\beta_{0}\right)>0$ small enough, there are no solutions of

$$
F_{\beta}\left(\omega \mid \begin{array}{l}
\left\{y_{a}\right\}_{1}^{n} \\
\left\{z_{a}\right\}_{1}^{n}
\end{array}\right)=0 \text { for } \omega \in U_{\delta} \text {, this uniformly in } 0 \leq n \leq m \text { and }\left(\beta,\left\{y_{a}\right\}_{1}^{n},\left\{z_{a}\right\}_{1}^{n}\right) \in \widetilde{\boldsymbol{U}}_{\beta_{0}} \times U_{\delta}^{n} \times U_{\delta}^{n} .
$$

It is clear that the optimal value of $\beta_{0}$ preventing the existence of such solutions depends on the width $\delta$ of the strip $U_{\delta}$ and on the integer $m$.

Hence, our strategy is as follows. We will always start our computations on a representation that is holomorphic in the half-plane $\Re \beta \geq 0$, as for instance (4.12)-(4.13). In the intermediate calculations whose purpose is to allow one to relate the initial representation to another one, we will assume that $\beta \in \widetilde{\boldsymbol{U}}_{\beta_{0}}$. This will allow us to avoid the problem of the aforementioned poles and represent $\widehat{\mathcal{G}}_{N ; \gamma}$ in terms of a regular functional that is moreover fit for carrying out the intermediate calculations. Then, once that we obtain the final expression, we will check 
that this new representation is in fact holomorphic in the half-plane $\mathfrak{R}(\beta) \geq 0$ and has thus a unique extension from $\widetilde{\boldsymbol{U}}_{\beta_{0}}$ up to $\beta=0$. As the same property holds for the initial representation, both will be equal at $\beta=0$.

Having agreed on such a prescription for dealing with the $\beta$-regularization and treating the $\beta \rightarrow 0$ limit, the effective form factor expansion-based representation for $\rho_{N ; \text { eff }}^{(m)}(x, t)$ (4.12) can be simplified with the use of the two properties below.

\section{The functional $\widehat{G}_{N ; \gamma}$}

Given $A \in \mathbb{R}^{+}$, we define the compact $K_{A}$ contained in $U_{\delta}$ :

$$
K_{A}=\{z \in \mathbb{C}:|\mathfrak{J} z| \leq \delta,|\mathfrak{R} z| \leq A\},
$$

and denote the open disk of radius $r$ by $\mathcal{D}_{0, r}=\{z \in \mathbb{C}:|z|<r\}$.

As follows from lemma A.2, given $A>0$ and large and $m \in \mathbb{N}^{*}$ fixed, there exists

- a complex number $\beta_{0}$ with a sufficiently large real part and an imaginary part small enough

- a positive number $\widetilde{\gamma}_{0}>0$ small enough

- a regular functional $\widehat{\mathscr{G}}_{\gamma ; A}^{(\beta)}$

such that, uniformly in $0 \leq n \leq m,\left(\gamma, \beta,\left\{\mu_{p_{a}}\right\}_{1}^{n},\left\{\mu_{h_{a}}\right\}_{1}^{n}\right) \in \mathcal{D}_{0, \widetilde{\gamma}_{0}} \times \widetilde{\boldsymbol{U}}_{\beta_{0}} \times K_{A}^{n} \times K_{A}^{n}$ one has

$$
\widehat{\mathcal{G}}_{N ; \gamma}\left(\begin{array}{l}
\left\{p_{a}\right\}_{1}^{n} \\
\left\{h_{a}\right\}_{1}^{n}
\end{array}\right)\left[\gamma F_{\beta} ; \xi ; \xi_{\gamma F_{\beta}}\right]=\widehat{\mathscr{G}}_{\gamma ; A}^{(\beta)}\left[H\left(* \mid \begin{array}{c}
\left\{\mu_{p_{a}}\right\}_{1}^{n} \\
\left\{\mu_{h_{a}}\right\}_{1}^{n}
\end{array}\right)\right] \quad \text { with } \quad H\left(\lambda \mid \begin{array}{l}
\left\{\mu_{p_{a}}\right\}_{1}^{n} \\
\left\{\mu_{h_{a}}\right\}_{1}^{n}
\end{array}\right)=\sum_{a=1}^{n} \frac{1}{\lambda-\mu_{p_{a}}}-\frac{1}{\lambda-\mu_{h_{a}}} .
$$

The $*$ in the argument of $\widehat{\mathscr{G}}_{\gamma ; A}^{(\beta)}$ appearing above indicates the running variable of $H$ on which this functional acts. The explicit expression for the functional $\widehat{\mathscr{G}}_{\gamma ; A}^{(\beta)}$ is given in lemma A.1, The main advantage of such a representation is that all the dependence on the auxiliary parameters is now solely contained in the function $H$ given in (4.28). The constant $\widetilde{\gamma}_{0}$ is such that

$$
\left|\gamma F_{\beta}\left(\omega \mid \begin{array}{l}
\left\{y_{a}\right\}_{1}^{n} \\
\left\{z_{a}\right\}_{1}^{n}
\end{array}\right)\right|<\frac{1}{2} \quad \text { uniformly in } \quad\left(\gamma, \beta,\left\{y_{a}\right\}_{1}^{n},\left\{z_{a}\right\}_{1}^{n}\right) \in \mathcal{D}_{0, \widetilde{\gamma}_{0}} \times \widetilde{\boldsymbol{U}}_{\beta_{0}} \times K_{A}^{n} \times K_{A}^{n} \text { and } 0 \leq n \leq m .
$$

The functional $\widehat{\mathscr{G}}_{\gamma ; A}^{(\beta)}$ is regular in respect to the to the pair $\left(M_{\mathscr{G}_{A}}, \mathscr{C}\left(K_{A}\right)\right)$ where $\mathscr{C}\left(K_{A}\right)$ in a loop in $U_{\delta}$ around $K_{A}$ as depicted in the $r h s$ of Fig. 1 and $M \mathscr{G}_{A}$ corresponds to the compact with one hole that is delimited by $\mathscr{C}_{\text {in }}$ and $\mathscr{C}_{\text {out }}$. This hole contains $K_{A}$. Finally, the parameters $\beta_{0} \in \mathbb{C}$ and $\widetilde{\gamma}_{0}>0$ are such that the constant of regularity $C_{\mathscr{G}_{A}}$ satisfies to the estimates

$$
C_{\mathscr{G}_{A}} \frac{\pi d\left(\partial M_{\mathscr{G}_{A}}, \mathscr{C}\left(K_{A}\right)\right)}{\left|\partial M_{\mathscr{G}_{A}}\right|+2 \pi d\left(\partial M_{\mathscr{G}_{A}}, \mathscr{C}\left(K_{A}\right)\right)}>A,
$$

where $\left|\partial M_{\mathscr{G}_{A}}\right|$ stands for the length of the boundary $\partial M_{\mathscr{G}_{A}}$ and $d\left(\partial M_{\mathscr{G}_{A}}, \mathscr{C}\left(K_{A}\right)\right)>0$ for the distance of $\mathscr{C}\left(K_{A}\right)$ to $\partial M_{\mathscr{G}_{A}}$.

Similarly to the discussion carried out in section 4.3.2 and according to proposition D.1, one has that, uniformly in $n, p \in\{0, \ldots, m\}$, and $z_{j}, y_{j}, j=1, \ldots, m$ belonging to $K_{A}$ :

$$
\frac{\partial^{p}}{\partial \gamma^{p}} \cdot \widehat{\mathscr{G}}_{\gamma ; A}^{(\beta)}\left[H\left(* \mid \begin{array}{c}
\left\{z_{j}\right\}_{1}^{n} \\
\left\{y_{j}\right\}_{1}^{n}
\end{array}\right)\right]_{\mid \gamma=0}=\lim _{r \rightarrow+\infty}\left\{\prod_{j=1}^{n} \mathrm{e}^{\widehat{g}_{2, r}\left(z_{j}\right)-\widehat{g}_{2, r}\left(y_{j}\right)} \cdot \frac{\partial^{p}}{\partial \gamma^{p}} \widehat{\mathscr{G}}_{\gamma ; A}^{(\beta)}\left[\varpi_{r}\right]\right\}_{\substack{\eta_{a, p}=0 \\
\gamma=0}}
$$


The compact $M \mathscr{G}_{A}$ has one hole. Hence, as discussed in section D.3 one has to consider two sets of discretization points $t_{1, p}, p=1, \ldots, r+1$ for $\mathscr{C}_{\text {in }}$ and $t_{2, p}, p=1, \ldots, r+1$ for $\mathscr{C}_{\text {out }}$. The function $\varpi_{r}$ appearing in (4.31) is a linear polynomial in the variables $\eta_{a, p}$ with $a=1,2$ and $p=1, \ldots, r$ :

$$
\varpi_{r}\left(\lambda \mid\left\{\eta_{a, p}\right\}\right)=\sum_{p=1}^{r} \frac{t_{1, p+1}-t_{1, p}}{2 i \pi\left(t_{1, p}-\lambda\right)} \eta_{1, p}+\sum_{p=1}^{r} \frac{t_{2, p+1}-t_{2, p}}{2 i \pi\left(t_{2, p}-\lambda\right)} \eta_{2, p} .
$$

Finally, $\widehat{g}_{2, r}(\lambda)$ is a differential operator in respect to $\eta_{a, p}$ with $a=1,2$ and $p=1, \ldots, r$ :

$$
\widehat{g}_{2, r}(\lambda)=\sum_{p=1}^{r} \frac{1}{t_{1, p}-\lambda} \frac{\partial}{\partial \eta_{1, p}}+\sum_{p=1}^{r} \frac{1}{t_{2, p}-\lambda} \frac{\partial}{\partial \eta_{2, p}} .
$$

\section{The functional $\widehat{D}_{N}$}

One can draw a small loop $\mathscr{C}_{\text {out }}$ around $K_{2 q}$ in $U_{\delta}$ as depicted in the lhs of Fig. 1. Let $M_{\widehat{D}}$ be the compact without holes whose boundary is delimited by $\mathscr{C}_{\text {out }}$. Then, given $L$ large enough, the functional $\widehat{D}_{N}$, as defined by (4.4), is a regular functional (in respect to the pair $\left(M_{\widehat{D}}, K_{2 q}\right)$ ) of $\gamma F_{\beta}$ with $\beta \in \widetilde{\boldsymbol{U}}_{\beta_{0}}$ and $|\gamma| \leq \widetilde{\gamma}_{0}$. The parameters $\beta_{0}$ and $\widetilde{\gamma}_{0}$ are as defined previously. This regularity is readily seen by writing down the integral representation:

$$
\lambda_{j}=\oint_{\partial K_{2 q}} \frac{\xi_{\gamma F_{\beta}}^{\prime}(\omega)}{\xi_{\gamma F_{\beta}}(\omega)-j / L} \frac{\mathrm{d} \omega}{2 i \pi}, \quad j=1, \ldots, N
$$

which holds provided that $L$ is large enough (indeed then all $\lambda_{j}$ 's are located in a very small vicinity of the interval $[-q ; q])$. Therefore, according to the results developed in appendix $\mathrm{D}$ and outlined in section 4.3, one has that, uniformly in $\beta \in \widetilde{\boldsymbol{U}}_{\beta_{0}}$ and $0 \leq p, n \leq m$

$$
\left.\left.\begin{array}{rl}
\frac{\partial^{p}}{\partial \gamma^{p}}\left\{\widehat { D } _ { N } ( \begin{array} { l } 
{ \{ p _ { a } \} _ { 1 } ^ { n } } \\
{ \{ h _ { a } \} _ { 1 } ^ { n } }
\end{array} ) \left[\gamma F_{\beta}\left(\cdot \mid \begin{array}{l}
\left\{\mu_{p_{a}}\right\}_{1}^{n} \\
\left\{\mu_{h_{a}}\right\}_{1}^{n}
\end{array}\right) ; \xi ; \xi_{\gamma F_{\beta}}\right.\right.
\end{array}\right]\right\}_{\mid \gamma=0}
$$

The function $\lambda \mapsto v_{s}(\lambda)$ appearing above is holomorphic in some open neighborhood of $K_{2 q}$ in $M_{\widehat{D}}$ and given by

$$
v_{s}\left(\lambda \mid\left\{\varsigma_{a}\right\}_{1}^{s}\right) \equiv v_{s}(\lambda)=(i \beta-1 / 2) Z(\lambda)-\phi(\lambda, q)+\sum_{j=1}^{s} \frac{\left(t_{j+1}-t_{j}\right)}{t_{j}-\lambda} \cdot \frac{\varsigma_{j}}{2 i \pi} .
$$

The parameters $t_{j}, j=1, \ldots, s$ correspond to a discretisation ( $c f$ definition 4.2) of the loop $\mathscr{C}_{\text {out }}$ around $K_{2 q}$ in $U_{\delta}$ that has been depicted in the lhs of Fig. $1 \varsigma_{j}$ are some sufficiently small complex numbers and $\widehat{g}_{1, s}(\lambda)$ is a differential operator in respect to $\varsigma_{a}$ :

$$
\widehat{g}_{1, s}(\lambda)=-\sum_{j=1}^{s} \phi\left(t_{j}, \lambda\right) \frac{\partial}{\partial \varsigma_{j}} .
$$


We remind that the parameters $\lambda_{a}$ appearing in the second line of (4.35) through the expression (4.4) for $\widehat{D}_{N}$, are the uniquef solutions to $\xi_{\gamma v_{s}}\left(\lambda_{a}\right)=a / L$. As such, the $\lambda_{a}$ 's become holomorphic functions of $\left\{S_{a}\right\}_{1}^{s}$ when these belong to a sufficiently small neighborhood of the origin in $\mathbb{C}^{s}$.

\section{Representation for the Taylor coefficients}

To implement the simplifications induced by the functional translations on the level of $\rho_{N ; \text { eff }}^{(m)}(x, t)$, we first observe that all of the rapidities $\mu_{p_{a}}$ and $\mu_{h_{a}}$ occurring in the course of summation in (4.12) belong to the interval [ $-A_{L} ; B_{L}$ ] with $L \xi\left(-A_{L}\right)=-w_{L}-1 / 2$ and $L \xi\left(B_{L}\right)=w_{L}+1 / 2\left(A_{L}>B_{L}\right)$. Hence, a fortiori, they belong to the compact $K_{2 A_{L}}$. We can thus represent the smooth part functional as $\widehat{\mathscr{G}}_{\gamma ; 2 A_{L}}^{(\beta)}$. We are interested solely in the $m^{\text {th }} \gamma$-derivative of (4.12) at $\gamma=0$. As $\widehat{D}_{N, n} \propto \gamma^{2(n-1)}$ and $\widehat{\mathscr{G}}_{\gamma ; 2 A_{L}}^{(\beta)}\left[\varpi_{r}\right]$ has no singularities around $\gamma=0$, all terms issuing from $n$ particle/hole excitations with $n \geq m$ will not contribute to the value of the derivative. Hence, we can truncate the sum over $n$ in (4.12) at $n=m$. Once that the sum is truncated, we represent the functional $\partial_{\gamma}^{m} \cdot\left\{\widehat{D}_{N} \cdot \widehat{\mathscr{G}}_{\gamma ; 2 A_{L}}^{(\beta)}\right\}_{\mid \gamma=0}$ with the help of identities (4.35) and (4.31). This leads to

$$
\begin{aligned}
& \rho_{N ; \mathrm{eff}}^{(m)}(x, t)=\lim _{\beta \rightarrow 0} \lim _{s \rightarrow+\infty} \lim _{r \rightarrow+\infty}\left[\sum_{\substack{n=0 \\
n=0}}^{m} \sum_{\substack{p_{1}<\cdots<p_{n} \\
p_{a} \in \mathcal{B}_{L}^{\text {ext }}}} \sum_{h_{1}<\cdots<h_{n}} \prod_{\substack{h_{a} \in \mathcal{B}_{L}^{\text {int }} \\
h^{\prime}}}^{n} \frac{\widehat{E}_{-1}^{2}\left(\mu_{h_{a}}\right)}{\widehat{E}_{-}^{2}\left(\mu_{p_{a}}\right)}\right. \\
& \left.\cdot \frac{\partial^{m}}{\partial \gamma^{m}}\left\{\widehat{D}_{N}\left(\begin{array}{l}
\left\{p_{a}\right\}_{1}^{n} \\
\left\{h_{a}\right\}_{1}^{n}
\end{array}\right)\left[\gamma v_{s} ; \xi ; \xi_{\gamma v_{s}}\right] \widehat{\mathscr{G}}_{\gamma ; 2 A_{L}}^{(\beta)}\left[\varpi_{r}\right]\right\}_{\substack{\gamma=0 \\
S_{p}=0=\eta_{a, p}}}\right] .
\end{aligned}
$$

We have set

$$
\widehat{E}_{-}^{2}(\lambda)=\mathrm{e}^{-i x u(\lambda)-\widehat{g}(\lambda)} \quad \text { with } \quad \widehat{g}(\lambda) \equiv \widehat{g}_{1, s}(\lambda)+\widehat{g}_{2, r}(\lambda)
$$

Above, in order to lighten the notation we have not written explicitly the dependence of $v_{s}, \varpi_{r}$ on the auxiliary parameters $\varsigma_{p}, \eta_{a, p}$ nor the one of $\widehat{E}_{-}(\lambda)$ on the discretization indices $r$ and $s$. However, we have kept the hat so as to insist on the operator valuedness of $\widehat{E}_{-}$. We do insist that (4.38) has to be understood as it was discussed in section 4.3 ,

Starting from representation (4.38), $\rho_{N \text {;eff }}^{(m)}(x, t)$ can be related with the $m^{\text {th }} \gamma$-derivative of the form factor like representation of the functional $X_{N}\left[\gamma v_{s}, \widehat{E}_{-}^{2}\right]$ given in $(\underline{B .20)}$. Namely, for such an identification to hold, one has to extend the upper bound in the summation over $n$ from $m$ up to $N+1$. This does not alter the result as it corresponds to adding up a finite amount of terms that are zero due to the presence of $\gamma$-derivatives. Then, one should use the identity

$$
\begin{gathered}
\frac{\partial^{m}}{\partial \gamma^{m}}\left\{\widehat{D}_{N}\left(\begin{array}{l}
\left\{p_{a}\right\}_{1}^{n} \\
\left\{h_{a}\right\}_{1}^{n}
\end{array}\right)\left[\gamma v_{s} ; \xi ; \xi_{\gamma v_{s}}\right] \widehat{\mathscr{G}}_{\gamma ; 2 A_{L}}^{(\beta)}\left[\varpi_{r}\right]\right\}_{\substack{\gamma=0 \\
S_{p}=0=\eta_{a, p}}} \\
=: \frac{\partial^{m}}{\partial \gamma^{m}}\left\{\frac{\prod_{a=1}^{N+1} \widehat{E}_{-}^{2}\left(\mu_{a}\right)}{\prod_{a=1}^{N} \widehat{E}_{-}^{2}\left(\lambda_{a}\right)} \cdot \frac{\prod_{a=1}^{N} \widehat{E}_{-}^{2}\left(\lambda_{a}\right)}{\prod_{a=1}^{N+1} \widehat{E}_{-}^{2}\left(\mu_{a}\right)} \widehat{D}_{N}\left(\begin{array}{l}
\left\{p_{a}\right\}_{1}^{n} \\
\left\{h_{a}\right\}_{1}^{n}
\end{array}\right)\left[\gamma v_{s} ; \xi ; \xi_{\gamma v_{s}}\right] \widehat{\mathscr{G}}_{\gamma ; 2 A_{L}}^{(\beta)}\left[\varpi_{r}\right]\right\}_{\substack{\gamma=0 \\
S_{p}=0=\eta_{a, p}}}:
\end{gathered}
$$

\footnotetext{
${ }^{\dagger}$ Here, as previously, the uniqueness follows from Rouché's theorem. By writing down an integral representation for $\xi_{\gamma v_{s}}^{-1}$, one readily convinces oneself that, for $\gamma$ small enough and given any fixed $s, \lambda_{a}$ is holomorphic in $\left\{\varsigma_{a}\right\}_{1}^{s}$. It is also holomorphic in $\gamma$ belonging to some open neighborhood of $\gamma=0$.

${ }^{\dagger}$ The latter is a functional of $v_{s}$ and $\widehat{g}$ as discussed in subsection 4.3 .2
} 
Just as it is the case for the parameters $\lambda_{j}$ appearing in the expression for $\widehat{D}_{N}$, the ones appearing in the pre-factors of the rhs in (4.40) are the unique solutions to $\xi_{\gamma \gamma_{s}}\left(\lambda_{s}\right)=s / L$. (4.40) is an expression of the type (4.22), and to deal correctly with it one should implement a : · : prescription for the way the differential operators $\partial_{\varsigma a}$ or $\partial_{\eta_{a, p}}$ should be substituted in the rhs of 44.40).

With the help of identity (4.40), one is able to force the appearance of the product of function $\widehat{E}_{-}$whose presence is necessary for identifying the sum over the particle-hole type labeling of integers in (4.38) with the functional $\partial_{\gamma}^{m} X_{N}\left[\gamma v_{s} \widehat{E}_{-}^{2}\right]_{\mid \gamma=0}$ given in $(\overline{B .20})$. This leads to the below representation:

$$
\rho_{N ; \text { eff }}^{(m)}(x, t)=\lim _{\beta \rightarrow 0} \lim _{s \rightarrow+\infty} \lim _{r \rightarrow+\infty}: \frac{\partial^{m}}{\partial \gamma^{m}}\left\{\frac{\prod_{a=1}^{N+1} \widehat{E}_{-}^{2}\left(\mu_{a}\right)}{\prod_{a=1}^{N} \widehat{E}_{-}^{2}\left(\lambda_{a}\right)} X_{N}\left[\gamma v_{s}, \widehat{E}_{-}^{2}\right] \widehat{\mathscr{G}}_{\gamma ; 2 A_{L}}^{(\beta)}\left[\varpi_{r}\right]\right\}_{\left.\right|_{S_{p}=0=\eta_{a, p}}=0}:
$$

\subsection{Taking the thermodynamic limit}

It is shown in appendix $\mathrm{C}$, theorem $\mathrm{C}$.1 1 that $\rho_{N \text {;eff }}^{(m)}(x, t)$ admits a well defined thermodynamic limit that we denote $\rho_{\mathrm{eff}}^{(m)}(x, t)$. This limit is given in terms of a multidimensional analogue of a (truncated) Fredholm series. This series is close in spirit to the type of series that have appeared in [45, 63]. It is also shown in that appendix (proposition C.1) that it is allowed to exchange

- the thermodynamic limit $N, L \rightarrow+\infty, N / L \rightarrow D$

with

- the $\partial_{\gamma}^{m}$ differentiation along with its associated operator substitution,

- the computation of the translation generated by $\widehat{g}_{2, r}$,

- the computation of the $s$-dimensional Lagrange series associated with $\widehat{g}_{1, s}$,

- the computation of the $r \rightarrow+\infty$ and $s \rightarrow+\infty$ limits,

- the analytic continuation in $\beta$ from $\mathfrak{R}(\beta)>\beta_{0}$ up to $\beta=0$.

The result of such an exchange of symbols is that $\rho_{\mathrm{eff}}^{(m)}(x, t)$ admits the representation

$$
\rho_{\mathrm{eff}}^{(m)}(x, t)=\lim _{w \rightarrow+\infty} \lim _{\beta \rightarrow 0} \lim _{s \rightarrow+\infty} \lim _{r \rightarrow+\infty}: \frac{\partial^{m}}{\partial \gamma^{m}}\left\{\widehat{E}_{-}^{2}(q) \cdot \mathrm{e}^{-\int_{-q}^{q}\left[i x u^{\prime}(\lambda)+\widehat{g}^{\prime}(\lambda)\right] \gamma \gamma_{s}(\lambda) \mathrm{d} \lambda} X_{\mathscr{C}_{E}^{(w)}}\left[\gamma v_{s}, \widehat{E}_{-}^{2}\right] \mathscr{G}_{\gamma ; 2 w}^{(\beta)}\left[\varpi_{r}\right]\right\} \underset{\mid \gamma=0}{:} .
$$

This formula deserves a few comments. In the case of complex valued functions $\widetilde{E}_{-}$, the functional $X_{\mathscr{C}_{E}^{(w)}}\left[\gamma v_{s}, \widetilde{E}_{-}^{2}\right]$ appearing in (4.42) corresponds to a Fredholm minor (B.34) of an integrable integral operator $I+V$ acting on $L^{2}([-q ; q])$. The kernel $V$ of this operator is given by $(\mathbb{B} .35)$.

The subscript $\mathscr{C}_{E}^{(w)}$ in $X_{\mathscr{C}_{E}^{(w)}}\left[\gamma v_{s}, \widetilde{E}_{-}^{2}\right]$ refers to an auxiliary compact contour entering in the definition of the kernel $V$. The parameter $w$ delimiting the size of this contour plays the role of a regularization. The limit of an unbounded contour $\mathscr{C}_{E}^{(\infty)}$ can only be taken after $r$ and $s$ are sent to infinity and the analytic continuation up to $\beta=0$ is carried out. Finally, in (4.42) also appears the functional $\mathscr{G}_{\gamma ; 2 w}^{(\beta)}$. It can be thought of as the thermodynamic limit of the functional $\widehat{\mathscr{G}}_{\gamma ; 2 w}^{(\beta)}$. Its precise expression and properties are discussed in lemma A.1.

We also would like to stress that the parameter $\beta_{0}$ defining the region $\widetilde{\boldsymbol{U}}_{\beta_{0}}$ from which one should carry out the analytic continuation up to $\beta=0$ depends on $2 w$ as stated in lemma.1. This dependence is chosen in such a way 


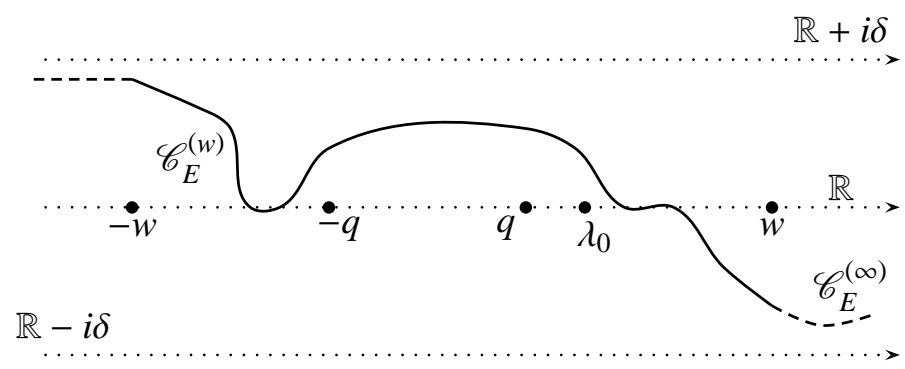

Figure 2: The contour $\mathscr{C}_{E}^{(w)}$ consists of the solid line. The contour $\mathscr{C}_{E}^{(\infty)}$ corresponds to the union of the solid and dotted lines. The localization of the saddle-point $\lambda_{0}$ corresponds to the space-like regime. Both contours lie in $U_{\delta / 2}$.

that that the constant of regularity $C_{\mathscr{G}_{A}}$ for the functional $\mathscr{G}_{\gamma ; 2 w}^{(\beta)}$ is large enough so as to make licit all the necessary manipulations with the translation operators and generalizations thereof.

We stress that formula (4.42) constitutes the most important result of appendix C Indeed, it provides one with a convenient representation for the thermodynamic limit $\rho_{\mathrm{eff}}^{(m)}(x, t)$. The latter constitutes the first step towards extracting the large-distance $x$ and long-time $t$ asymptotic behavior of $\rho_{\mathrm{eff}}^{(m)}(x, t)$. The proof of such a representation for the thermodynamic limit is however quite technical and lengthy. It can definitely be skipped on a first reading. Moreover, should one be solely interested in a "short path" to extracting the asymptotics, we stress that formula (4.42) can be readily obtained without the use of any complicated and long computations. It is enough to take the thermodynamic limit formally on the level of formula (4.41). Such a formal manipulation leads straightforwardly to the representation (4.42).

\subsection{The multidimensional Natte series and asymptotics}

Theorem 4.1 The thermodynamic limit of the Taylor coefficients $\rho_{\mathrm{eff}}^{(m)}(x, t)$ admits the below truncated multidimensional Natte series representation

$$
\begin{aligned}
& \rho_{\mathrm{eff}}^{(m)}(x, t)=\frac{\partial^{m}}{\partial \gamma^{m}}\left\{\frac{\mathbf{1}_{q ;+\infty}\left[\left(\lambda_{0}\right)\right.}{\sqrt{-2 \pi x u^{\prime \prime}\left(\lambda_{0}\right)}} \times \frac{\mathrm{e}^{i x\left[u\left(\lambda_{0}\right)-u(q)\right]} \mathcal{B}\left[\gamma F_{q}^{\lambda_{0}} ; p\right] \mathcal{A}_{0}\left[\gamma F_{q}^{\lambda_{0}}\right]}{\left(x-t v_{F}+i 0^{+}\right)^{\left[\gamma F_{q}^{\lambda_{0}}(q)\right]^{2}}\left(x+t v_{F}\right)^{\left[\gamma F_{q}^{\lambda_{0}}(-q)\right]^{2}}} \mathcal{G}_{1 ; \gamma}^{(0)}\left(\begin{array}{c}
\lambda_{0} \\
q
\end{array}\right)\right. \\
& +\frac{\mathrm{e}^{i x[u(-q)-u(q)]}\left(\mathcal{B} \mathcal{A}_{-}\right)\left[\gamma F_{q}^{-q} ; p\right] \mathcal{G}_{1 ; \gamma}^{(0)}\left(\begin{array}{c}
-q \\
q
\end{array}\right)}{\left(x-t v_{F}+i 0^{+}\right)^{\left[\gamma F_{q}^{-q}(q)\right]^{2}}\left(x+t v_{F}\right)^{\left[\gamma F_{q}^{-q}(-q)-1\right]^{2}}}+\frac{\left(\mathcal{B} \mathcal{A}_{+}\right)\left[\gamma F_{\emptyset}^{\emptyset} ; p\right] \mathcal{G}_{0 ; \gamma}^{(0)}\left(\begin{array}{c}
\emptyset \\
\emptyset
\end{array}\right)}{\left(x-t v_{F}+i 0^{+}\right)^{\left[\gamma F_{\emptyset}^{\emptyset}(q)+1\right]^{2}}\left(x+t v_{F}\right)^{\left[\gamma F_{\emptyset}^{0}(-q)\right]^{2}}} \\
& \left.+\mathrm{e}^{-i x u(q)} \sum_{n=1}^{m} \sum_{\mathcal{K}_{n}} \sum_{\mathcal{E}_{n}(\vec{k})} \int_{\mathscr{C}_{\epsilon_{t}}^{(w)}} \frac{H_{n ; x}^{\left(\left\{\epsilon_{t}\right\}\right)}\left(\left\{u\left(z_{t}\right)\right\} ;\left\{z_{t}\right\}\right)\left[\gamma F_{z_{-}}^{z_{+}}\right] \mathcal{B}\left[\gamma F_{z_{-}}^{z_{+}} ; p\right]}{\left(x-i 0^{+}\right)^{\left[\gamma F_{z-}^{z+}(q)\right]^{2}}\left(x+t v_{F}\right)^{\left[\gamma F_{z-}^{z_{-}}(-q)\right]^{2}}} \mathcal{G}_{\mid\left\{z_{+}\right\} ; ; \gamma}^{(0)}\left(\begin{array}{c}
\left\{z_{+}\right\} \\
\left\{z_{-}\right\}
\end{array}\right) \frac{\mathrm{d}^{n} z_{t}}{(2 i \pi)^{n}}\right\}_{\mid \gamma=0} \text {. }
\end{aligned}
$$

There, we have introduced the notations

$$
\left\{z_{+}\right\}=\left\{z_{\boldsymbol{t}}, \boldsymbol{t} \in J_{\{\vec{k}\}}: \epsilon_{t}=1\right\}, \quad\left\{z_{-}\right\}=\left\{z_{\boldsymbol{t}}, \boldsymbol{t} \in J_{\{\vec{k}\}}: \epsilon_{t}=-1\right\} \cup\{q\}, \quad\left|\left\{z_{+}\right\}\right| \equiv \#\left\{z_{\boldsymbol{t}}, \boldsymbol{t} \in J_{\{\vec{k}\}}: \epsilon_{t}=1\right\} .
$$


$F_{\emptyset}^{\emptyset}, F_{q}^{\lambda_{0}}, F_{q}^{-q}$ have been defined in (3.5) and, in general, we agree upon

$$
F_{z_{-}}^{z_{+}}(\lambda) \equiv F\left(\lambda \mid \begin{array}{l}
\left\{z_{+}\right\} \\
\left\{z_{-}\right\}
\end{array}\right)=-\frac{Z(\lambda)}{2}-\sum_{\substack{t \in J_{\{k\}} \\
\epsilon_{t}=1}} \phi\left(\lambda, z_{t}\right)+\sum_{\substack{t \in J_{\{k\}} \\
\epsilon_{t}=-1}} \phi\left(\lambda, z_{t}\right) .
$$

The function $\mathcal{G}_{n ; \gamma}^{(0)}$ is related to the thermodynamic limit of the smooth part of the form factor. Its expression can be found in (A.8). The functionals $\mathcal{B}, \mathcal{A}_{ \pm}$and $\mathcal{A}_{0}$ are given by

$$
\mathcal{B}[v, p]=\frac{[\varkappa[v](-q)]^{\nu(-q)}}{[\varkappa[v](q)]^{\nu(q)}} \frac{G^{2}(1+v(q)) G^{2}(1-v(-q)) \mathrm{e}^{i \frac{\pi}{2}\left(\nu^{2}(q)-v^{2}(-q)\right)}}{\left[2 q p^{\prime}(q)\right]^{\nu^{2}(q)}\left[2 q p^{\prime}(-q)\right]^{\nu^{2}(-q)}(2 \pi)^{v(q)-v(-q)}} \mathrm{e}^{\frac{1}{2} \int_{-q}^{q} \frac{v^{\prime}(\lambda) v(\mu)-\nu^{\prime}(\mu) v(\lambda)}{\lambda-\mu} \mathrm{d} \lambda \mathrm{d} \mu},
$$

where $G$ is the Barnes double Gamma function,

$$
\mathcal{A}_{+}[v, p]=\frac{-2 q \varkappa^{-2}[v](q)}{\left[2 q p^{\prime}(q)\right]^{2 v(q)+1}} \Gamma\left(\begin{array}{c}
1+v(q) \\
-v(q)
\end{array}\right) \frac{1}{\mathrm{e}^{-2 i \pi v(q)}-1} \quad, \quad x[v](\lambda)=\exp \left\{-\int_{-q}^{q} \frac{v(\lambda)-v(\mu)}{\lambda-\mu} \mathrm{d} \mu\right\},
$$

and

$$
\mathcal{A}_{-}[v, p]=\frac{-2 q}{\varkappa^{2}[v](-q)} \Gamma\left(\begin{array}{c}
1-v(-q) \\
v(-q)
\end{array}\right) \frac{\left[2 q p^{\prime}(-q)\right]^{2 v(-q)-1}}{\mathrm{e}^{-2 i \pi v(-q)}-1} \quad \text { and } \quad \mathcal{A}_{0}[v]=\mathrm{e}^{-i \frac{\pi}{4}} \mathcal{\varkappa}^{-2}[v]\left(\lambda_{0}\right)\left(\frac{\lambda_{0}-q}{\lambda_{0}+q}\right)^{2 v\left(\lambda_{0}\right)}
$$

The second sum appearing in the last line of (4.43) runs through all the elements $\vec{k}$ belonging to

$$
\mathcal{K}_{n}=\left\{\vec{k}=\left(k_{1}, \ldots, k_{n+1}\right): k_{n+1} \in \mathbb{N}^{*} \text { and } k_{a} \in \mathbb{N}, a=1, \ldots, n \text { such that } \sum_{a=1}^{n} a k_{a}+k_{n+1}=n\right\} .
$$

Once that an element of $\mathcal{K}_{n}$ has been fixed, one defines the associated set of triplets $J_{\{\vec{k}\}}$ :

$$
J_{\{\vec{k}\}}=\left\{\left(t_{1}, t_{2}, t_{3}\right), t_{1} \in \llbracket 1 ; n+1 \rrbracket, t_{2} \in \llbracket 1 ; k_{t_{1}} \rrbracket, t_{3} \in \mathbb{[} 1 ; t_{1}-n \delta_{t_{1}, n+1} \rrbracket\right\} .
$$

The third sum runs through all the elements $\left\{\epsilon_{t}\right\}_{t \in J_{\{\vec{k}\}}}$ belonging to the set

$$
\mathcal{E}_{n}(\vec{k})=\left\{\left\{\epsilon_{t}\right\}_{t \in J_{\{\vec{k}\}}}: \epsilon_{t} \in\{ \pm 1,0\} \forall t \in J_{\{\vec{k}\}} \quad \text { with } \quad \sum_{t_{3}=1}^{t_{1}} \epsilon_{t}=0 \quad \text { for } \boldsymbol{t}_{1}=1, \ldots, n \text { and } \sum_{p=1}^{k_{n+1}} \epsilon_{n+1, p, 1}=1\right\} .
$$

In other words, $\mathcal{E}_{n}(\vec{k})$ consists of $n$-uples of parameters $\epsilon_{t}$ labeled by triplets $\boldsymbol{t}=\left(\boldsymbol{t}_{1}, \boldsymbol{t}_{2}, \boldsymbol{t}_{3}\right)$ belonging to $J_{\{\vec{k}\}}$. Each element of such an $n$-uple takes its values in $\{ \pm 1,0\}$. In addition, the components of this $n$-uple are subject to summation constraints. These hold for any value of $\boldsymbol{t}_{1}$ or $\boldsymbol{t}_{2}$ and are different whether one deals with $\boldsymbol{t}_{1}=1, \ldots, n$ or with $\boldsymbol{t}_{1}=n+1$.

The integral appearing in the $n^{\text {th }}$ summand occurring in the third line of 4.43 is $n$-fold. The contours of integration $\mathscr{C}_{\epsilon_{t}}^{(w)}$ depend on the choices of elements in $\mathcal{E}_{n}(\vec{k})$ and are realized as $n$-fold Cartesian products of onedimensional compact curves that correspond to various deformations of the base curve $\mathscr{C}_{E}^{(w)}$ depicted in Fig. 2 In the $w \rightarrow+\infty$ limit, these curves go to analogous deformations of the base curve $\mathscr{C}_{E}^{(\infty)}$. All these contours lie in $U_{\delta / 2}$

The integrand $H_{n ; x}^{\left(\left\{\epsilon_{t}\right\}\right)}\left(\left\{u\left(z_{t}\right)\right\} ;\left\{z_{t}\right\}\right)[v]$ is a regular functional of $v$, that is simultaneously a function of $u\left(z_{t}\right)$ and $z_{\boldsymbol{t}}$ with $\boldsymbol{t}$ running through the set $J_{\{\vec{k}\}}$. This functional depends on the choice of an element $\left\{\epsilon_{\boldsymbol{t}}\right\}_{t \in J_{\{\vec{k}\}}}$ from $\mathcal{E}_{n}(\vec{k})$ and on $x$. It appears originally as a building block of the Natte series (cf appendix B.5 for more details). 
We stress that all summands involving the functional $\mathcal{G}_{n ; \gamma}^{(\beta)}$ are well defined at $\beta=0$. The potential singularities present in $\mathcal{G}_{n ; \gamma}^{(\beta)}$ are canceled by the zeroes of the pre-factors.

Proof-

As a starting point for the proof, we need to introduce the below set of functions depending on the auxiliary parameters $a_{p}, b_{1, p}$ and $b_{2, p}$. As it has been discussed in section 4.3.2, these functions will allow us to compute the (functional) coefficients necessary for carrying out the operator substitution. We set

$$
\widetilde{E}_{-}^{2}(\lambda)=\mathrm{e}^{-i x u(\lambda)-\widetilde{g}(\lambda)} \quad \text { with } \quad \widetilde{g}(\lambda)=\widetilde{g}_{1, s}(\lambda)+\widetilde{g}_{2, r}(\lambda),
$$

where

$$
\widetilde{g}_{1, s}(\lambda)=-\sum_{p=1}^{s} \phi\left(t_{p}, \lambda\right) a_{p} \quad \text { and } \quad \widetilde{g}_{2, r}(\lambda)=\sum_{p=1}^{r} \frac{b_{1, p}}{t_{1, p}-\lambda}+\sum_{p=1}^{r} \frac{b_{2, p}}{t_{2, p}-\lambda} .
$$

It is readily checked with the help of lemma A.1 and proposition B.3

that for $\gamma$ small enough $\mathcal{F}$ given below is a regular functional of $v_{s}, \varpi_{r}$ and $\widetilde{g}$ :

$$
\mathcal{F}\left[\gamma v_{s}, \widetilde{g}, \varpi_{r}\right](\gamma)=\widetilde{E}_{-}^{2}(q) \cdot \mathrm{e}^{-\int_{-q}^{q}\left[i x u^{\prime}(\lambda)+\widetilde{g}^{\prime}(\lambda)\right] \gamma v_{s}(\lambda) \mathrm{d} \lambda} X_{\mathscr{C}_{E}^{(w)}}\left[\gamma v_{s}, \widetilde{E}_{-}^{2}\right] \mathscr{G}_{\gamma ; 2 w}^{(\beta)}\left[\varpi_{r}\right] .
$$

In particular $\mathcal{F}\left[\gamma v_{s}, \widetilde{g}, \varpi_{r}\right](\gamma)$ is holomorphic in $\gamma$, at least for $\gamma$ small enough. In order to implement the operator substitution, we have to compute the Taylor coefficients of the series expansion of $\mathcal{F}\left[\gamma v_{s}, \widetilde{g}, \varpi_{r}\right](\gamma)$ into powers of $b_{1, p}, b_{2, p}$ with $p=1, \ldots, r$ and $a_{p}$ with $p=1, \ldots, s$. These Taylor coefficients are solely determined by the functional $\mathcal{F}\left[\gamma v_{s}, \widetilde{g}, \varpi_{r}\right](\gamma)$ depending on the classical function $\widetilde{g}$ (4.19). Therefore, one can use any equivalent representation for $\mathcal{F}\left[\gamma v_{s}, \widetilde{g}, \varpi_{r}\right](\gamma)$ as a starting point for computing the various partial derivatives in respect to $b_{j, p}$ or $a_{p}$. In other words, one can use any equivalent series representation $]^{\dagger}$ for the Fredholm minor $X_{\mathscr{C}_{E}^{(w)}}\left[\gamma v_{s}, \widetilde{E}_{-}^{2}\right]$. Clearly, different series representations for the Fredholm minor will lead to different type of expressions for the Taylor coefficients. However, in virtue of the uniqueness of the Taylor coefficients, their values coincide. As shown in [62], the Fredholm minor we're interested in admits the so-called Natte series representation. The latter series of multiple integrals is built in such a way that it gives a quasi-direct access to the asymptotic behavior of $X_{\mathscr{C}_{E}^{(w)}}\left[\gamma v, \widetilde{E}_{-}^{2}\right]$. It is thus clear that this is THE series representation that is fit for providing the large-distance/longtime asymptotic expansion of the two-point function. We will thus take this series representation as a starting point for our calculations.

The first remarkable consequence of the use of the Natte series is that the exponential pre-factor in front of $X_{\mathscr{C}_{E}^{(w)}}\left[\gamma v_{s}, \widetilde{E}_{-}^{2}\right]$ in (4.53) exactly compensates the one appearing in the Natte series $(\overline{B .48})$. Once that these prefactors are simplified, one should take the $m^{\text {th }} \gamma$-derivative of the remaining part of the Natte series representation (B.48) for $X_{\mathscr{C}_{E}^{(w)}}\left[\gamma v_{s}, \widetilde{E}_{-}^{2}\right] \mathscr{G}_{\gamma ; 2 w}^{(\beta)}\left[\varpi_{r}\right]$. One of the consequences of taking the $m^{\text {th }}-\gamma$ derivative is that the Natte series

\footnotetext{
${ }^{\dagger}$ One natural representation that can be used as a starting point for taking the derivatives is the Fredholm series-like representation for $X_{\mathscr{C}_{E}^{(w)}}\left[\gamma v_{s}, \widetilde{E}_{-}^{2}\right]$. In fact, it is this series representation that has been used for the computations carried out in theroem C.1.
} 
given in $(\overline{B .48})$ becomes truncated at $n=m$ due to the property $i i)$ of the functions $H_{n ; x}^{(\{\epsilon\})}(c f$ appendix B.5]:

$$
\begin{aligned}
& \frac{\partial^{m}}{\partial \gamma^{m}} \mathcal{F}\left[\gamma v_{s}, \widetilde{g}, \varpi_{r}\right](\gamma)_{\mid \gamma=0}=\mathrm{e}^{i x\left[u\left(\lambda_{0}\right)-u(q)\right]} \mathrm{e}^{\widetilde{g}\left(\lambda_{0}\right)-\widetilde{g}(q)} \frac{\partial^{m}}{\partial \gamma^{m}}\left\{\frac{\mathcal{B}\left[\gamma v_{s} ; u+i 0^{+}\right] \mathcal{A}_{0}\left[\gamma v_{s}\right]}{\sqrt{-2 \pi u^{\prime \prime}\left(\lambda_{0}\right) x} \cdot x^{\gamma^{2} v_{s}^{2}(q)+\gamma^{2} v_{s}^{2}(-q)}} \mathscr{G}_{\gamma ; 2 w}^{(\beta)}\left[\varpi_{r}\right]\right\}_{\mid \gamma=0} \times \mathbf{1}_{q ;+\infty[}\left(\lambda_{0}\right) \\
& +\mathrm{e}^{i x[u(-q)-u(q)]} \mathrm{e}^{\widetilde{g}(-q)-\widetilde{g}(q)} \frac{\partial^{m}}{\partial \gamma^{m}}\left\{\frac{\left(\mathcal{B} \mathcal{A}_{-}\right)\left[\gamma \nu_{s} ; u+i 0^{+}\right]}{x^{\left(1-\gamma v_{s}(-q)\right)^{2}+\gamma^{2} v_{s}^{2}(q)}} \mathscr{G}_{\gamma ; 2 w}^{(\beta)}\left[\varpi_{r}\right]\right\}_{\mid \gamma=0}+\frac{\partial^{m}}{\partial \gamma^{m}}\left\{\frac{\left(\mathcal{B} \mathcal{A}_{+}\right)\left[\gamma v_{s} ; u+i 0^{+}\right]}{x^{\gamma^{2} v_{s}^{2}(-q)+\left(\gamma v_{s}(q)+1\right)^{2}}} \mathscr{G}_{\gamma ; 2 w}^{(\beta)}\left[\varpi_{r}\right]\right\}_{\mid \gamma=0} \\
& +\mathrm{e}^{-\widetilde{g}(q)-i x u(q)} \sum_{n=1}^{m} \sum_{\mathcal{K}_{n}} \sum_{\mathcal{E}_{n}\left(\left\{k_{a}\right\}\right)} \oint_{\mathscr{C}_{\epsilon_{t}}^{(w)}} \prod_{\boldsymbol{t} \in J_{\{k\}}}\left\{\mathrm{e}^{\epsilon_{\mathrm{t}} \widetilde{g}\left(z_{t}\right)}\right\} \cdot \frac{\partial^{m}}{\partial \gamma^{m}}\left\{H_{n ; x}^{\left(\left\{\epsilon_{t}\right\}\right)}\left(\left\{u\left(z_{t}\right)\right\} ;\left\{z_{t}\right\}\right)\left[\gamma v_{s}\right] \frac{\mathcal{B}\left[\gamma v_{s} ; u+i 0^{+}\right]}{x^{\gamma^{2} v_{s}^{2}(q)+\gamma^{2} \mathcal{V}_{s}^{2}(-q)}} \mathscr{G}_{\gamma ; 2 w}^{(\beta)}\left[\varpi_{r}\right]\right\}_{\mid \gamma=0} \frac{\mathrm{d}^{n} z_{\boldsymbol{t}}}{(2 i \pi)^{n}} .
\end{aligned}
$$

It follows from lemma $\mathrm{A} .1$ representation $(\mathrm{B.50})$ and the explicit formulae for the functionals $\mathcal{B}, \mathcal{A}_{0}$ and $\mathcal{A}_{ \pm}$ (4.46)-(4.48) that the functionals occurring in (4.54) are all regular ( $c f$ definition 4.1). Moreover, as follows from the previous discussion relative to the procedure of taking the $\beta \rightarrow 0$ limit, at this stage of the calculations, $\mathfrak{R}(\beta)>0$ is large enough so that the constant of regularity $C_{\mathscr{G}_{2 w}}$ of the functionals $\mathscr{G}_{\gamma ; 2 w}^{(\beta)}$ is sufficiently large to be able to apply proposition D.1 and corollary D.1 (due to the estimates (4.30) for $C_{\mathscr{G}_{2 w}}$, the constant $\gamma_{0}$ occurring in (D.4) is greater then 1 for $w$ large enough, which is the limit of interest) to this functional. proposition D.1 and corollary D.1 are also directly applicable to all functionals of $\gamma v_{s}$ in as much as, at the end of the day, one sets $\gamma=0$.

Clearly, there is no problem to implement the substitution $a_{p} \mapsto \partial_{S_{p}}$ and $b_{i, p} \mapsto \partial_{\eta_{i, p}}$ on the level of (4.54) in such a way that all the partial derivative operators appear to the left of all $\eta_{i, p}$ and $\varsigma_{p}$ dependent functions. The first two lines in (4.54) will give rise to translation operators. In the case of the ultimate line in (4.54), this operator substitution will produce expressions of the type

$$
\begin{aligned}
\sum_{n_{p} \geq 0}^{+\infty} \sum_{n_{a, p} \geq 0}^{+\infty} \prod_{p=1}^{s}\left\{\frac{1}{n_{p} !}\right. & \left.\frac{\partial^{n_{p}}}{\partial \varsigma_{p}^{n_{p}}}\right\} \prod_{p=1}^{r} \prod_{a=1}^{2}\left\{\frac{1}{\left(n_{a, p}\right) !} \frac{\partial^{n_{a, p}}}{\partial \eta_{a, p}^{n_{a, p}}}\right\} \oint_{\mathscr{C}_{\epsilon_{t}(w)}} \prod_{p=1}^{s}\left[\Omega_{p}\left(\left\{z_{t}\right\}\right)\right]^{n_{p}} \prod_{p=1}^{r} \prod_{a=1}^{2}\left[\Omega_{a, p}^{\prime}\left(\left\{z_{\boldsymbol{t}}\right\}\right)\right]^{n_{a, p}} \\
& \times \frac{\partial^{m}}{\partial \gamma^{m}}\left\{H_{n, x}^{\left(\left\{\epsilon_{t}\right\}\right)}\left(\left\{u\left(z_{t}\right)\right\} ;\left\{z_{t}\right\}\right)\left[\gamma v_{s}\right] \frac{\mathcal{B}\left[\gamma v_{s} ; u+i 0^{+}\right]}{x^{\gamma^{2} \nu_{s}^{2}(q)+\gamma^{2} \nu_{s}^{2}(-q)}} \mathscr{G}_{\gamma ; 2 w}^{(\beta)}\left[\varpi_{r}\right]\right\}_{\substack{\gamma=0 \\
S_{p}=0=\eta_{a, p}}} \frac{\mathrm{d}^{n} z_{t}}{(2 i \pi)^{n}} .
\end{aligned}
$$

Where $\Omega_{p}$ and $\Omega_{a, p}^{\prime}$ take the form

$$
\Omega_{p}\left(\left\{z_{t}\right\}\right)=\phi\left(t_{p}, q\right)-\sum_{t \in J_{\{k\}}} \epsilon_{t} \phi\left(t_{p}, z_{t}\right) \quad \text { and } \quad \Omega_{a, p}^{\prime}\left(\left\{z_{t}\right\}\right)=\frac{1}{t_{a, p}-q}-\sum_{t \in J_{\{k\}}} \epsilon_{t} \frac{1}{t_{a, p}-z_{t}} .
$$

One can compute the $r, s \rightarrow+\infty$ limit of such series of integrals by applying corollary 7 .1 and observing that $\mathscr{C}_{\epsilon_{t}}^{(w)}$ is a Cartesian product of a finite number of compact one dimensional curves that are contained in $U_{\delta / 2}$. In fact, the result of this corollary allows one to carry out the operator substitution in (4.54) directly under the integration sign. In other words, one is allowed to replace $\widetilde{g}_{1, s} \hookrightarrow \widehat{g}_{1, s}$ and $\widetilde{g}_{2, r} \hookrightarrow \widehat{g}_{2, r}$ directly on the level of (4.54), this without pulling out the partial $\varsigma_{p}$ or $\eta_{a, p}$ derivatives out of the integrals. Hence, one is brought to computing the action of translation operators. The latter can be estimated by applying proposition D.1 Again, there is no problem to apply this proposition either because we compute the $m^{\text {th }} \gamma$-derivative at $\gamma=0$ (so that $\gamma$ can be as small as desired in the case of functionals of $\gamma v_{s}$ ) or because the constant of regularity is large enough for $\mathscr{G}_{\gamma ; 2 w}^{(\beta)}$. As follows from this proposition, one can permute the partial $\gamma$-derivative symbols at $\gamma=0$ with the

\footnotetext{
${ }^{\dagger}$ This corollary can be applied to $\mathscr{G}_{\gamma ; 2 w}^{(\beta)}$ precisely because its constant of regularity is large enough.
} 
action of the finite $s$ and $r$ translation operators. It then remains to take the $r \rightarrow+\infty$ and the $s \rightarrow+\infty$ limits. As in each case the convergence is uniform, the limit can be taken directly under the finite sum, compact integrals and partial $\gamma$-derivatives symbols.

Then, in order to compute the effect of the $s \rightarrow+\infty$ limit we apply the identity (4.15) (also $c f$ appendix D.3):

$$
\lim _{s \rightarrow+\infty} \prod_{a=1}^{n} \mathrm{e}^{\widehat{g}_{1, s}\left(z_{a}\right)-\widehat{g}_{1, s}\left(y_{a}\right)} \cdot \mathscr{F}\left[\gamma v_{s}\right]=\mathscr{F}\left[\gamma F_{\beta}\right] \quad \text { with } \quad F_{\beta}(\lambda) \equiv F_{\beta}\left(\lambda \mid \begin{array}{l}
\left\{z_{a}\right\}_{1}^{n} \\
\left\{y_{a}\right\}_{1}^{n}
\end{array}\right),
$$

valid for any regular functional $\mathscr{F},|\gamma|$ small enough and $z_{a}, y_{a}$ all lying in $U_{\delta}$. Here, we would like to remind that $F_{\beta}$ appearing above correspond to the thermodynamic limit of the $\beta$-deformed shift function, $c f(2.11)$. Similarly,

$$
\lim _{r \rightarrow+\infty} \prod_{a=1}^{n} \mathrm{e}^{\widehat{\alpha}_{2, r}\left(z_{a}\right)-\widehat{g}_{2, r}\left(y_{a}\right)} \cdot \mathscr{G}_{\gamma ; 2 w}^{(\beta)}\left[\varpi_{r}\right]=\mathscr{G}_{\gamma ; 2 w}^{(\beta)}\left[H\left(\cdot \mid \begin{array}{c}
\left\{z_{a}\right\}_{1}^{n} \\
\left\{y_{a}\right\}_{1}^{n}
\end{array}\right)\right] \quad \text { with } \quad H\left(\lambda \mid \begin{array}{l}
\left\{z_{a}\right\}_{1}^{n} \\
\left\{y_{a}\right\}_{1}^{n}
\end{array}\right)=\sum_{a=1}^{n} \frac{1}{\lambda-z_{a}}-\frac{1}{\lambda-y_{a}} .
$$

All this for $\left(\left\{z_{a}\right\}_{1}^{n} ;\left\{y_{a}\right\}_{1}^{n}\right) \in K_{2 w}^{2 n}$. Then, by applying lemma A.1 backwards, we get

$$
\mathscr{G}_{\gamma ; 2 w}^{(\beta)}\left[H\left(\cdot \mid \begin{array}{l}
\left\{z_{a}\right\}_{1}^{n} \\
\left\{y_{a}\right\}_{1}^{n}
\end{array}\right)\right]=\mathcal{G}_{n ; \gamma}^{(\beta)}\left(\begin{array}{c}
\left\{z_{a}\right\}_{1}^{n} \\
\left\{y_{a}\right\}_{1}^{n}
\end{array}\right) .
$$

The function $\mathcal{G}_{n ; \gamma}^{(\beta)}$ has been defined in (A.8).

Therefore, we obtain

$$
\begin{gathered}
\rho_{\mathrm{eff}}^{(m)}(x, t)=\lim _{w \rightarrow+\infty} \lim _{\beta \rightarrow 0} \frac{\partial^{m}}{\partial \gamma^{m}}\left\{\frac{\mathcal{B}\left[\gamma \widetilde{F}_{q}^{\lambda_{0}} ; u+i 0^{+}\right] \mathcal{A}_{0}\left[\gamma \widetilde{F}_{q}^{\lambda_{0}}\right]}{\sqrt{-2 \pi u^{\prime \prime}\left(\lambda_{0}\right) x}} \frac{\mathrm{e}^{i x\left[u\left(\lambda_{0}\right)-u(q)\right]}}{{ }_{x}^{\left[\gamma \widetilde{F}_{q}^{\lambda_{0}}(q)\right]^{2}+\left[\gamma \widetilde{F}_{q}^{\lambda_{0}}(-q)\right]^{2}} \mathcal{G}_{1 ; \gamma}^{(\beta)}\left(\begin{array}{c}
\lambda_{0} \\
q
\end{array}\right)}\right. \\
+\frac{\mathrm{e}^{i x[u(-q)-u(q)]}}{x^{\left[\gamma \widetilde{F}_{q}^{-q}(q)\right]^{2}+\left[1-\gamma \widetilde{F}_{q}^{-q}(-q)\right]^{2}}} \cdot\left(\mathcal{B} \mathcal{A}_{-}\right)\left[\gamma \widetilde{F}_{q}^{-q} ; u+i 0^{+}\right] \mathcal{G}_{1 ; \gamma}^{(\beta)}\left(\begin{array}{c}
-q \\
q
\end{array}\right) \\
+\frac{\left(\mathcal{B} \mathcal{A}_{+}\right)\left[\gamma \widetilde{F}_{\emptyset}^{\emptyset} ; u+i 0^{+}\right]}{x^{\left[1+\gamma \widetilde{F}_{\emptyset}^{0}(q)\right]^{2}+\left[\gamma \widetilde{F}_{\emptyset}^{0}(-q)\right]^{2}}} \mathcal{G}_{0 ; \gamma}^{(\beta)}\left(\begin{array}{c}
\emptyset \\
\emptyset
\end{array}\right)+\mathrm{e}^{-i x u(q)} \sum_{n=1}^{m} \sum_{\mathcal{K}_{n}} \sum_{\mathcal{E}_{n}\left(\left\{k_{a}\right\}\right)} \oint_{\mathscr{C}_{\epsilon_{t}}^{(w)}} \frac{\mathcal{B}\left[\gamma \widetilde{F}_{z_{-}}^{z_{+}} ; u+i 0^{+}\right]}{{ }^{\left[\gamma \widetilde{F}_{z-}^{z+}(q)\right]^{2}+\left[\gamma \widetilde{F}_{z-}^{z+}(-q)\right]^{2}}} \\
\left.\times H_{n ; x}^{\left(\left\{\epsilon_{t}\right\}\right)}\left(\left\{u\left(z_{t}\right)\right\} ;\left\{z_{t}\right\}\right)\left[\gamma \widetilde{F}_{z_{-}}^{z_{+}}\right] \mathcal{G}_{\left|\left\{z_{+}\right\}\right| ; \gamma}^{(\beta)}\left(\begin{array}{c}
\left\{z_{+}\right\} \\
\left\{z_{-}\right\}
\end{array}\right) \frac{\mathrm{d}^{n} z_{t}}{(2 i \pi)^{n}}\right\}_{\mid \gamma=0} .
\end{gathered}
$$

Here $\widetilde{F}_{z_{-}}^{z_{+}}(\lambda)=F_{z_{-}}^{z_{+}}(\lambda)+i \beta Z(\lambda)$ and $F_{z_{-}}^{z_{+}}$has been defined in (4.45).

Once that the functional translations have been computed, one should carry out the analytic continuation of the expression in brackets from $\beta \in \widetilde{\boldsymbol{U}}_{\beta_{0}}$ up to $\beta=0$ and then send $w$ to $+\infty$. For this, we recall that the functions $H_{n ; x}^{\left(\left(\epsilon_{\epsilon}\right\}\right)}$ admit the below decomposition $(c f(\overline{\mathrm{B} .50})$ :

$H_{n ; x}^{\left(\left\{\epsilon_{t}\right\}\right)}\left(\left\{u\left(z_{t}\right)\right\} ;\left\{z_{t}\right\}\right)\left[\gamma \widetilde{F}_{z_{-}}^{z_{+}}\right]=\widetilde{H}_{n ; x}^{\left(\left\{\epsilon_{t}\right\}\right)}\left(\left\{\gamma \widetilde{F}_{z_{-}}^{z_{+}}\left(z_{t}\right)\right\},\left\{u\left(z_{t}\right)\right\},\left\{z_{t}\right\}\right) \prod_{t \in J_{\{k\}}}\left(\varkappa\left[\widetilde{F}_{z_{-}}^{z_{+}}\right]\left(z_{t}\right)\right)^{-2 \epsilon_{t}} \prod_{z_{t} \in\left\{z_{+}\right\}}\left(\mathrm{e}^{-2 i \pi \gamma \widetilde{F}_{z_{-}}^{z_{+}}\left(z_{t}\right)}-1\right)^{2}$.

It follows from the way $H_{n ; x}^{\left(\left\{\epsilon_{t}\right)\right.}$ depends on the set of its $v$-type arguments (4.61) and from the expression for the functional $\mathcal{B}[v, u](4.46)$ and $\mathcal{G}_{n ; \gamma}^{(\beta)}(\mathrm{A} .8)$ that all of the expressions one deals with contain the combination

$$
G^{2}\left(1-\gamma \widetilde{F}_{z_{-}}^{z_{+}}(-q)\right) G^{2}\left(1+\gamma \widetilde{F}_{z_{-}}^{z_{+}}(q)\right) \prod_{z_{t} \in\left\{z_{+}\right\}}\left(\mathrm{e}^{-2 i \pi \gamma \widetilde{F}_{--}^{z_{+}}\left(z_{t}\right)}-1\right)^{2} \operatorname{det}_{\mathscr{C}_{q}}\left[I+\gamma \mathcal{V}\left[\widetilde{F}_{z_{-}}^{z_{+}}, \widetilde{F}_{z_{-}}^{z_{+}}\right]\right] \operatorname{det} \mathscr{C}_{q}\left[I+\gamma \overline{\mathcal{V}}\left[\widetilde{F}_{z_{-}}^{z_{+}}, \widetilde{F}_{z_{-}}^{z_{+}}\right]\right]
$$


In virtue of proposition A.1, the function appearing above is holomorphic in $\left(\beta, \gamma,\left\{z_{+}\right\},\left\{z_{-}\right\}\right) \in\{\mathfrak{R}(\beta) \geq 0\} \times$ $\overline{\mathcal{D}}_{0,1} \times U_{\delta}^{\left|\left\{z_{+}\right\}\right|} \times U_{\delta}^{\left|\left\{z_{+}\right\}\right|}$.

The function $\widetilde{H}_{n ; x}^{\left(\left\{\epsilon_{t}\right)\right)}\left(\left\{\gamma \widetilde{F}_{z_{-}}^{z_{+}}\left(z_{t}\right)\right\},\left\{u\left(z_{t}\right)\right\},\left\{z_{t}\right\}\right)$ is analytic in $(\gamma, \beta) \in \mathcal{D}_{0, \widetilde{\gamma}_{0}} \times \widetilde{\boldsymbol{U}}_{\beta_{0}}$ (here $\widetilde{\gamma}_{0}$ is chosen so that $\left|\gamma \widetilde{F}_{z_{-}}^{z_{+}}\left(z_{t}\right)\right|<1 / 2$ uniformly in the variables $z_{\boldsymbol{t}}, \boldsymbol{t} \in J_{\{\vec{k}\}}$ belonging to $\left.U_{\delta}\right)$, and integrable in respect to the $\left\{z_{t}\right\}_{t \in J_{\left\{k_{a}\right\}}}$ variables. The remaining part of $\mathcal{G}_{n ; \gamma}^{(\beta)}$ has also the same properties. As the integrals are compactly supported it follows that the whole expression appearing inside of the "big" brackets in (4.60) is holomorphic in $(\gamma, \beta) \in$ $\mathcal{D}_{0, \widetilde{\gamma}_{0}} \times \widetilde{\boldsymbol{U}}_{\beta_{0}}$. As a consequence, the $m^{\text {th }} \gamma$-derivative at $\gamma=0$ can be continued up to $\beta=0$. To get the value of the analytic continuation at this point it is in fact enough to set $\beta=0$ in (4.60).

The last step consists in taking the limit $w \rightarrow+\infty$. This operation will result in an extension of the integration contours from bounded ones $\mathscr{C}_{\epsilon_{t}}^{(w)}$ to ones going to infinity $\mathscr{C}_{\epsilon_{t}}^{(\infty)}$. Hence, one needs to check that the resulting integrals will be convergent. Note that the function $F_{z_{-}}^{z_{+}}\left(z_{t}\right)$ are bounded whenever $z_{t}$ or any of the variables belonging to the set $\left\{z_{+}\right\}$or $\left\{z_{-}\right\}$goes to infinity. Also, the function $\mathcal{G}_{n ; 1}^{(\beta)}$ is bounded at infinity by a polynomial in $z_{t}$ of degree $n$, this uniformly in respect to $\gamma$-derivatives of order $0, \ldots, m$. Therefore, as the functions $\widetilde{H}_{n ; x}^{\left.\left(1 \epsilon_{t}\right\}\right)}\left(\left\{\widetilde{F}_{z_{-}}^{z_{+}}\left(z_{t}\right)\right\},\left\{u\left(z_{t}\right)\right\},\left\{z_{t}\right\}\right)$ go to zero exponentially fast in all directions where $\mathscr{C}_{\epsilon_{t}}^{(\infty)}$ goes to $\infty$, the integrals over $\mathscr{C}_{\epsilon_{t}}^{(\infty)}$ are indeed convergent.

\subsection{Some more conjectures leading to the dominant asymptotics of $\rho(x, t)$}

Under the assumption that

1. the Taylor series $\sum_{m=0}^{+\infty} \gamma^{m} \rho_{\mathrm{eff}}^{(m)}(x, t) / m$ ! is convergent up to $\gamma=1$,

2. its sum gives $\rho(x, t)$,

3. the multidimensional Natte series given below is convergent.

We get that $\rho(x, t)$ is obtained from (4.43) by removing the $m^{\text {th }} \gamma$-derivative symbol and setting $\gamma=1$. It then remains to identify the coefficients in the first two lines with the properly normalized thermodynamic limit of form factors of the field as given in (A.46), A.47) and (A.48). One then obtains the below series of multiple integral representation for the thermodynamic limit of the one-particle reduced density matrix:

$$
\begin{aligned}
& \rho(x, t)=\sqrt{\frac{-2 i \pi}{t \varepsilon^{\prime \prime}\left(\lambda_{0}\right)-x p^{\prime \prime}\left(\lambda_{0}\right)}} \times \frac{p^{\prime}\left(\lambda_{0}\right) \mathrm{e}^{i x\left[u\left(\lambda_{0}\right)-u(q)\right]}\left|\mathcal{F}_{q}^{\lambda_{0}}\right|^{2}}{\left[-i\left(x-t v_{F}\right)\right]^{\left[F_{q}^{\lambda_{0}}(q)\right]^{2}}\left[i\left(x+t v_{F}\right)\right]^{\left[F_{q}^{\lambda_{0}}(-q)\right]^{2}}} \mathbf{1}_{q ;+\infty}\left[\left(\lambda_{0}\right)\right. \\
& +\frac{\mathrm{e}^{-2 i x p_{F}}\left|\mathcal{F}_{q}^{-q}\right|^{2}}{\left[-i\left(x-t v_{F}\right)\right]^{\left[F_{q}^{-q}(q)\right]^{2}}\left[i\left(x+t v_{F}\right)\right]^{\left[F_{q}^{-q}(-q)-1\right]^{2}}}+\frac{\left|\mathcal{F}_{\emptyset}^{\emptyset}\right|^{2}}{\left[-i\left(x-t v_{F}\right)\right]^{\left[F_{\emptyset}^{\emptyset}(q)+1\right]^{2}}\left[i\left(x+t v_{F}\right)\right]^{\left[F_{\emptyset}^{\emptyset}(-q)\right]^{2}}} \\
& +\mathrm{e}^{-i x u(q)} \sum_{n=1}^{+\infty} \sum_{\mathcal{K}_{N}} \sum_{\mathcal{E}_{N}(\vec{k})} \oint_{\mathscr{C}_{\epsilon_{t}}^{(w)}} \mathcal{G}_{\mid\left\{z_{+}\right\} ; ; 1}^{(0)}\left(\begin{array}{c}
\left\{z_{+}\right\} \\
\left\{z_{-}\right\}
\end{array}\right) \frac{H_{n ; x}^{\left(\left\{\epsilon_{t}\right\}\right)}\left(\left\{u\left(z_{t}\right)\right\} ;\left\{z_{t}\right\}\right)\left[F_{z_{-}}^{z_{+}}\right] \mathcal{B}\left[F_{z_{-}}^{z_{+}} ; p\right]}{\left(x-t v_{F}+i 0^{+}\right)^{\left[z_{z-}^{z+}(q)\right]^{2}}\left(x+t v_{F}\right)^{\left[z_{z-}^{z+}(-q)\right]^{2}}} \cdot \frac{\mathrm{d}^{n} z_{t}}{(2 i \pi)^{n}} .
\end{aligned}
$$

It follows from the above representation and from conjecture B.1 that

Corollary 4.1 The reduced density matrix admits the asymptotic expansion as given in subsection 3.2

Proof- 
The proof is immediate as far as the multidimensional Natte series defining $\rho(x, t)$ is convergent. Indeed, then, the fine structure of the functions $H_{n ; x}^{\left(\left\{\epsilon_{t}\right\}\right)}$ given in (B.51) implies that all the contributions stemming from integrations are subdominant in respect to the first two lines in (4.62), this provided that $\left|F_{z_{-}}^{z_{+}}( \pm q)\right|<1 / 2$ for all configurations of variables in $\left\{z_{ \pm}\right\}$that belong to $\left\{\lambda_{0}, \pm q\right\}$. This condidtion is not satisfied, especially if $\left|\left\{z_{+}\right\}\right|$ becomes large. One should then invoke conjecture B.1 stating that, in fact, higher order oscillating terms in the representation (B.51) for $H_{n ; x}^{\left(\left\{\epsilon_{t}\right\}\right)}$ are more dampen than it is apparent from the sum in (B.51). This is enough to show that, indeed, the different type of higher oscillating tems stemming from $H_{n ; x}^{\left.\left(i \epsilon_{t}\right\}\right)}$ are subdominant in respect to the first two lines of (4.62). Actually, in this way, one recovers the whole expected tower of critical exponents for the terms corresponding purely to oscillations at integer multiples of $u(q)-u(-q)$ as predicted in [2] on the basis of CFT-based technique.

\section{Conclusion}

In this article, we have continued developing a new method allowing one to build two types of series of multiple integral representation for the correlation functions of integrable models starting from their form factor expansion. One of these series which we called the multidimensional Natte series yields a straightforward access to the large-distance/long-time asymptotic behavior of the two-point functions. In this way, we were able to extract the long-time/large-distance asymptotic behavior of the reduced density matrix for the non-linear Schrödinger model.

In order to provide applications to physically pertinent cases, the method we have developed has to recourse to a few conjectures. The first one is relative to the convergence of the series of multiple integrals representing the correlators. This conjecture is supported by the free fermion case, where the convergence is rather quick, especially in the large-distance/long-time regime. The second conjecture concerns the possibility of using an effective series instead of the one appearing in the form factors expansion of two-point functions. Both series have been assumed to have the same thermodynamic limit $N, L \rightarrow+\infty$. This conjecture is supported, on the physical ground, by the argument that sums over states whose energies scale as some power of the system-size ought to give a vanishing contribution to the sum over form factors once that the thermodynamic limit is taken. It would be very interesting and important from the conceptual point of view to prove these two conjectures in the case of models that are away from their free fermion points.

However, we do insist that we have organized the analysis in such a way that all of the aforementioned convergence issues are separated from the asymptotic analysis part. Therefore, all the part of this work related purely to the asymptotic analysis is rigorous. Moreover, we do expect that the scheme of asymptotic analysis we have developed can be applied in full rigor to many cases which are free of convergence issues. We do also stress that, for the moment, the proofs of convergence of series of multiple integral representations for correlation functions of models away from their free-fermion point are, in general, an open problem. Apart from very specific representations related to the spin-1/2 XXZ chain, the proof of convergence of a series representation for two-point functions could have been carried out only in the case of the Lee-Yang model by F. Smirnov.

We have chosen to develop our method on the example of the one-particle reduced density matrix in the nonlinear Schrödinger model. The case of the current-to-current correlation functions in this model will appear in [65]. It seems however that the method is quite general and applicable to a vast class of integrable models where the form factors of local operators are known. In particular, it should be applicable not only to lattice models where the form factors admit determinant-like representations [14, 53, 61, 73] but also to integrable field theories where the form factors of local operators can be computed through the resolution of the so-called bootstrap program. For instance, the method seems applicable to the analysis of certain two-point functions (and their short-distance asymptotics) in the sine-Gordon model whose form factors have been obtained in [81, 82]. In the latter case, we expect to deal with some multidimensional deformation of the $3^{\text {rd }}$ Painleve transcendent, a new type of special function whose description and asymptotic behavior is interesting in its own right. 


\section{Acknowledgment}

I acknowledge the support of the EU Marie-Curie Excellence Grant MEXT-CT-2006-042695. I would like to thank Kitanine, Maillet, Slavnov and Terras for numerous stimulating discussions.

\section{A Thermodynamic limit of the Form Factors of conjugated fields}

\section{A.1 Thermodynamic limit of form factors}

It has been shown in [64] with the help of techniques introduced in [46, 78] that the normalized modulus squared of the form factor of the conjugated field taken between the ground state $\left\{\lambda_{a}\right\}_{1}^{N}$ and any finite $n$ particle/hole type excited state $\left\{\mu_{\ell_{a}}\right\}_{1}^{N+1}$ admits the below behavior in the thermodynamic limit $N, L \rightarrow+\infty, N / L \rightarrow D$

$$
\frac{\left|\left\langle\psi\left(\left\{\mu_{\ell_{a}}\right\}_{1}^{N+1}\right)\left|\Phi^{\dagger}(0,0)\right| \psi\left(\{\lambda\}_{1}^{N}\right)\right\rangle\right|^{2}}{\left\|\psi\left(\left\{\mu_{\ell_{a}}\right\}_{1}^{N+1}\right)\right\|^{2}\left\|\psi\left(\{\lambda\}_{1}^{N}\right)\right\|^{2}}=D_{0 ; L}\left[F_{0}\right] \mathcal{R}_{N, n}\left(\begin{array}{c}
\left\{\mu_{p_{a}}\right\}_{1}^{n} ;\left\{p_{a}\right\}_{1}^{n} \\
\left\{\mu_{h_{a}}\right\}_{1}^{n} ;\left\{h_{a}\right\}_{1}^{n}
\end{array}\right)\left[F_{0}\right] \mathcal{G}_{n ; 1}^{(0)}\left(\begin{array}{c}
\left\{\mu_{p_{a}}\right\}_{1}^{n} \\
\left\{\mu_{h_{a}}\right\}_{1}^{n}
\end{array}\right)\left(1+\mathrm{O}\left(\frac{\ln L}{L}\right)\right) \text {. }
$$

Above, $F_{0}$ corresponds to the thermodynamic limit of the shift function associated to the excited state of interest (at $\beta=0$ ). The auxiliary parameters of $F_{0}$ are undercurrent by the various functionals appearing above. The parameters $\mu_{k}$ are defined as the unique solutions to $\xi\left(\mu_{a}\right)=a / L$.

\section{The discreet part}

The first two functionals appearing in (A.1) correspond to the leading in $L$ behavior of the so-called singular part $\widehat{D}_{N}\left[\widehat{F}_{\left\{\ell_{a}\right\}}, \widehat{\xi}_{\left\{\ell_{a}\right\}}, \widehat{\xi}\right]$ of the form factor, namely

$$
\widehat{D}_{N}\left(\begin{array}{c}
\left\{p_{a}\right\}_{1}^{n} \\
\left\{h_{a}\right\}_{1}^{n}
\end{array}\right)\left[\widehat{F}_{\left\{\ell_{a}\right\}}, \widehat{\xi}_{\left\{\ell_{a}\right\}}, \widehat{\xi}\right]=D_{0 ; L}\left[F_{0}\right] \mathcal{R}_{N, n}\left(\begin{array}{c}
\left\{\mu_{p_{a}}\right\}_{1}^{n} ;\left\{p_{a}\right\}_{1}^{n} \\
\left\{\mu_{h_{a}}\right\}_{1}^{n} ;\left\{h_{a}\right\}_{1}^{n}
\end{array}\right)\left[F_{0}\right]\left(1+\mathrm{O}\left(\frac{\ln L}{L}\right)\right) .
$$

Given any function $v(\lambda)$ holomorphic in some neighborhood of $[-q ; q]$, one has

$$
D_{0 ; L}[v]=\frac{[\varkappa[v](-q)]^{v(-q)}}{[\varkappa[v](q)]^{v(q)+2}} \prod_{a=1}^{n}\left(\frac{\lambda_{N+1}-\mu_{p_{a}}}{\lambda_{N+1}-\mu_{h_{a}}}\right)^{2} \frac{q G^{2}(1-v(-q)) G^{2}(2+v(q)) / \pi}{(2 \pi)^{v(q)-v(-q)} \cdot\left[2 q L \xi^{\prime}(q)\right]^{(v(q)+1)^{2}+v^{2}(-q)}} \cdot e^{\frac{1}{2} \int_{-q}^{q} \frac{v^{\prime}(\lambda) v(\mu)-v^{\prime}(\mu) v(\lambda)}{\lambda-\mu} \mathrm{d} \lambda \mathrm{d} \mu},
$$

The parameter $\lambda_{N+1}$ appearing above is defined as the unique solution to $L \xi_{v}\left(\lambda_{N+1}\right)=N+1, \varkappa[v](\lambda)$ is given by (4.47) and $G$ stands for the Barnes double Gamma function. Finally, we agree upon

$$
\begin{gathered}
\mathcal{R}_{N, n}\left(\begin{array}{c}
\left\{\mu_{p_{a}}\right\}_{1}^{n} ;\left\{p_{a}\right\}_{1}^{n} \\
\left\{\mu_{h_{a}}\right\}_{1}^{n} ;\left\{h_{a}\right\}_{1}^{n}
\end{array}\right)[v]=\prod_{a=1}^{n}\left\{\frac{\varphi\left(\mu_{h_{a}}, \mu_{h_{a}}\right) \varphi\left(\mu_{p_{a}}, \mu_{p_{a}}\right) \mathrm{e}^{\mathrm{N}[v]\left(\mu_{p_{a}}\right)}}{\varphi\left(\mu_{p_{a}}, \mu_{h_{a}}\right) \varphi\left(\mu_{h_{a}}, \mu_{p_{a}}\right) \mathrm{e}^{\mathrm{N}[v]\left(\mu_{h_{a}}\right)}}\right\} \frac{\prod_{a<b}^{n} \varphi^{2}\left(\mu_{p_{a}}, \mu_{p_{b}}\right) \varphi^{2}\left(\mu_{h_{a}}, \mu_{h_{b}}\right)}{\prod_{a \neq b}^{n} \varphi^{2}\left(\mu_{p_{a}}, \mu_{h_{b}}\right)} \operatorname{det}_{n}^{2}\left[\frac{1}{h_{a}-p_{b}}\right] \\
\times \prod_{a=1}^{n}\left(\frac{\sin \left[\pi v\left(\mu_{h_{a}}\right)\right]}{\pi}\right)^{2} \cdot \prod_{a=1}^{n} \Gamma^{2}\left(\begin{array}{c}
p_{a}-N-1+v\left(\mu_{p_{a}}\right), p_{a}, N+2-h_{a}-v\left(\mu_{h_{a}}\right), h_{a}+v\left(\mu_{h_{a}}\right) \\
p_{a}-N-1, p_{a}+v\left(\mu_{p_{a}}\right), N+2-h_{a}, h_{a}
\end{array}\right) .
\end{gathered}
$$

There

$$
\boldsymbol{\aleph}[v](\omega)=2 v(\omega) \ln \left(\frac{\varphi(\omega, q)}{\varphi(\omega,-q)}\right)+2 \int_{-q}^{q} \frac{v(\lambda)-v(\omega)}{\lambda-\omega} \mathrm{d} \lambda \quad \text { and } \quad \varphi(\lambda, \mu)=2 \pi \frac{\lambda-\mu}{p(\lambda)-p(\mu)} .
$$


Above, we have used the standard hypergeometric-type representation for products of $\Gamma$-functions:

$$
\Gamma\left(\begin{array}{l}
a_{1}, \ldots, a_{n} \\
b_{1}, \ldots, b_{n}
\end{array}\right)=\prod_{k=1}^{n} \frac{\Gamma\left(a_{k}\right)}{\Gamma\left(b_{k}\right)} .
$$

\section{Description of $\mathcal{G}_{n ; 1}^{(\beta)}$}

In order to give an explicit representation for $\mathcal{G}_{n ; \gamma}^{(\beta)}$ we need to introduce a few notations. First, let $F_{\beta}$ correspond to the thermodynamic limit of the $\beta$-deformed shift function associated to the choice of the rapidities $\left\{\mu_{p_{a}}\right\}$ for the particles and $\left\{\mu_{h_{a}}\right\}$ for the holes. The auxiliary arguments of the shift function will be kept undercurrent. Also, let $m \in \mathbb{N}$ and $U_{\delta}$ be the open strip (2.15) around $\mathbb{R}$.

Then there exists $\widetilde{\gamma}_{0}>0$ small enough and $\beta_{0} \in \mathbb{C}$ with $\mathfrak{R}\left(\beta_{0}\right)>0$ large enough and $\mathfrak{J}\left(\beta_{0}\right)>0$ small enough such that

$$
\mathrm{e}^{2 i \pi \gamma F_{\beta}(\omega)}-1 \neq 0 \quad \text { uniformly in } n=0, \ldots, m \quad \text { and } \quad\left(\gamma, \omega, \beta,\left\{\mu_{p_{a}}\right\}_{1}^{n},\left\{\mu_{h_{a}}\right\}_{1}^{n}\right) \in \mathcal{D}_{0, \widetilde{\gamma}_{0}} \times U_{\delta} \times \widetilde{\boldsymbol{U}}_{\beta_{0}} \times U_{\delta}^{n} \times U_{\delta}^{n} \text {. }
$$

Let all parameters $\mu_{h_{a}}, a=1, \ldots, n$ belong to a compact $K_{q+\epsilon} \supset[-q ; q]$ for some $\epsilon>0$ and let $\mathscr{C}_{q+\epsilon}$ be a small counterclockwise loop around this compact $K_{q+\epsilon}$, then $\mathcal{G}_{n, \gamma}^{(\beta)}$ admits the below representation

$$
\begin{aligned}
\mathcal{G}_{n ; \gamma}^{(\beta)}\left(\begin{array}{l}
\left\{\mu_{p_{a}}\right\}_{1}^{n} \\
\left\{\mu_{h_{a}}\right\}_{1}^{n}
\end{array}\right)=\mathrm{e}^{-2 i \pi \sum_{\epsilon= \pm} C\left[\gamma F_{\beta}\right](q+\epsilon i c)} \prod_{a=1}^{n} \prod_{\epsilon= \pm}\left\{\frac{\mu_{h_{a}}-q+\epsilon i c}{\mu_{p_{a}}-q+\epsilon i c} \frac{\mathrm{e}^{2 i \pi C\left[\gamma F_{\beta}\right]\left(\mu_{h_{a}}+\epsilon i c\right)}}{\mathrm{e}^{2 i \pi C\left[\gamma F_{\beta}\right]\left(\mu_{p a}+\epsilon i c\right)}}\right\} \mathrm{e}^{C_{0}\left[\gamma F_{\beta}\right]} \\
\quad \times \prod_{a, b=1}^{n} \frac{\left(\mu_{p_{a}}-\mu_{h_{b}}-i c\right)\left(\mu_{h_{a}}-\mu_{p_{b}}-i c\right)}{\left(\mu_{p_{a}}-\mu_{p_{b}}-i c\right)\left(\mu_{h_{a}}-\mu_{h_{b}}-i c\right)} \cdot \frac{\operatorname{det}_{\mathscr{C}_{q+\epsilon}}\left[I+\gamma \mathcal{V}\left[\gamma F_{\beta}, \gamma F_{\beta}\right]\right] \operatorname{det} \mathscr{C}_{q+\epsilon}\left[I+\gamma \overline{\mathcal{V}}\left[\gamma F_{\beta}, \gamma F_{\beta}\right]\right]}{\operatorname{det}^{2}[I-K / 2 \pi]} .
\end{aligned}
$$

There $C\left[F_{\beta}\right]$ stands for the Cauchy transform on $[-q ; q]$ and $C_{0}\left[F_{\beta}\right]$ is given by a double integral

$$
C\left[F_{\beta}\right](\lambda)=\int_{-q}^{q} \frac{\mathrm{d} \mu}{2 i \pi} \frac{F_{\beta}(\mu)}{\mu-\lambda} \quad \text { and } \quad C_{0}\left[F_{\beta}\right]=-\int_{-q}^{q} \frac{F_{\beta}(\lambda) F_{\beta}(\mu)}{(\lambda-\mu-i c)^{2}} \mathrm{~d} \lambda \mathrm{d} \mu
$$

The integral kernels $\mathcal{V}$ and $\overline{\mathcal{V}}$ read

$$
\mathcal{V}[v, \rho]\left(\omega, \omega^{\prime}\right)=\frac{1}{2 \pi} \frac{\omega-q}{\omega-q+i c} \prod_{a=1}^{n}\left\{\frac{\left(\omega-\mu_{p_{a}}\right)\left(\omega-\mu_{h_{a}}+i c\right)}{\left(\omega-\mu_{h_{a}}\right)\left(\omega-\mu_{p_{a}}+i c\right)}\right\} \cdot \mathrm{e}^{C[2 i \pi v](\omega)-C[2 i \pi v](\omega+i c)} \frac{K\left(\omega-\omega^{\prime}\right)}{\mathrm{e}^{-2 i \pi \rho(\omega)}-1}
$$

and

$$
\overline{\mathcal{V}}[v, \rho]\left(\omega, \omega^{\prime}\right)=\frac{-1}{2 \pi} \frac{\omega-q}{\omega-q-i c} \prod_{a=1}^{n}\left\{\frac{\left(\omega-\mu_{p_{a}}\right)\left(\omega-\mu_{h_{a}}-i c\right)}{\left(\omega-\mu_{h_{a}}\right)\left(\omega-\mu_{p_{a}}-i c\right)}\right\} \cdot \mathrm{e}^{C[2 i \pi v](\omega)-C[2 i \pi v](\omega-i c)} \frac{K\left(\omega-\omega^{\prime}\right)}{\mathrm{e}^{2 i \pi \rho(\omega)}-1}
$$

The representation (A.8) is valid for $n=0, \ldots, m$ and $\left(\gamma, \beta,\left\{\mu_{p_{a}}\right\}_{1}^{n},\left\{\mu_{h_{a}}\right\}_{1}^{n}\right) \in \overline{\mathcal{D}}_{0, \gamma_{0}} \times \widetilde{\boldsymbol{U}}_{\beta_{0}} \times U_{\delta}^{n} \times K_{q+\epsilon}^{n}$ and defines a holomorphic function of these parameters belonging to this set.

It is also valid at $\gamma=1$, provided that $\mathfrak{R}\left(\beta_{0}\right)>0$ is taken large enough for condition (A.7) to be fulfilled at $\gamma=1$.

Finally as follows from proposition $\mathrm{A}$.1 given below, the product $\mathcal{D}_{0 ; L}\left[F_{\beta}\right] \mathcal{R}_{N, n}\left[F_{\beta}\right] \mathcal{G}_{n ; 1}^{(\beta)}$ is holomorphic in $\mathfrak{R} \beta \geq 0$, and can thus be analytically continued from $\widetilde{\boldsymbol{U}}_{\beta_{0}}$ up to $\beta=0$. It is in this sense that the formula (A.1) for the leading asymptotics in the size $L$ of the form factors of $\Phi^{\dagger}$ is to be understood. 


\section{Proposition A.1 64}

Let $m \in \mathbb{N}, \delta>0$ small enough define the width of the strip $U_{\delta}$ around $\mathbb{R}$ and $\left(\left\{\mu_{p_{a}}\right\}_{1}^{n} ;\left\{\mu_{h_{a}}\right\}_{1}^{n}\right) \in U_{\delta}^{n} \times K_{q+\epsilon}^{n}$, where $\epsilon>0$ and the compact $K_{q+\epsilon}$ is as defined by (4.27).

Let $v, h$ and $\tau$ be holomorphic function in the strip $U_{\delta}$ around $\mathbb{R}$ and such that $h\left(U_{\delta}\right) \subset\{z: \mathbb{R}(z)>0\}$ and $\mathfrak{J}(h(z))$ is bounded on $U_{\delta}$. Set $v_{\beta}(\lambda)=v(\lambda)+i \beta h(\lambda)$.

Then, there exists

- $\beta_{0} \in \mathbb{C}$ with $\mathfrak{R}\left(\beta_{0}\right)>0$ large enough and $\mathfrak{I}\left(\beta_{0}\right)>0$ small enough

- $\widetilde{\gamma_{0}}>0$ and small enough

- a small loop $\mathscr{C}_{q+\epsilon} \subset U_{\delta}$ around the compact $K_{q+\epsilon}$

such that uniformly in $\beta \in \widetilde{\boldsymbol{U}}_{\beta_{0}}$ the function $\lambda \mapsto \mathrm{e}^{-2 i \pi \gamma(v+i \beta h)(\lambda)}-1$ has no roots inside of $\mathscr{C}_{q+\epsilon}$. In addition, the function

$\left(\left\{\mu_{p_{a}}\right\}_{1}^{n},\left\{\mu_{h_{a}}\right\}_{1}^{n}, \gamma, \beta\right) \mapsto G(1-\gamma \tau(-q)) G(2+\gamma \tau(q)) \prod_{a=1}^{n}\left(\mathrm{e}^{-2 i \pi \gamma v_{\beta}\left(\mu_{h_{a}}\right)}-1\right) \cdot \operatorname{det}_{\mathscr{C}_{q}}\left[I+\gamma \mathcal{V}\left[\gamma \tau, \gamma v_{\beta}\right]\left(\left\{\mu_{p_{a}}\right\}_{1}^{n},\left\{\mu_{h_{a}}\right\}_{1}^{n}\right)\right]$

is holomorphic functions in $U_{\delta}^{n} \times K_{q+\epsilon}^{n} \times \mathcal{D}_{0, \widetilde{\gamma}_{0}} \times \widetilde{\boldsymbol{U}}_{\beta_{0}}$, this uniformly in $0 \leq n \leq m$.

It admits a (unique) analytic continuation to $U_{\delta}^{n} \times K_{q+\epsilon}^{n} \times \overline{\mathcal{D}}_{0,1} \times\{z \in \mathbb{C}: \mathfrak{R}(z) \geq 0\}$. In particular, it has a well defined $\beta \rightarrow 0$ limit. The $\beta \rightarrow 0$ limit of this analytic continuation is still holomorphic in $\left(\left\{\mu_{p_{a}}\right\}_{1}^{n},\left\{\mu_{h_{a}}\right\}_{1}^{n}\right) \in$ $U_{\delta}^{n} \times K_{q+\epsilon}^{n}$.

In (A.12) we have insisted explicitly on the dependence of the integral kernel $\mathcal{V}$ on the auxiliary parameters $\left(\left\{\mu_{p_{a}}\right\}_{1}^{n},\left\{\mu_{h_{a}}\right\}_{1}^{n}\right), c f($ A.10). The same proposition holds when the kernel $\mathcal{V}$ is replaced by $\overline{\mathcal{V}}$ as it has been defined in (A.11).

\section{Alternative representation for $\mathcal{G}_{n ; \gamma}^{(\beta)}$}

It so happens that the smooth part of the form factor's asymptotics admits a representation as a functional acting on a unique function $H$. More precisely,

Lemma A.1 Let $m \in \mathbb{N}$ and the strip $U_{\delta}$ be fixed. Let $A>0$ be some constant defining the size of the compact $K_{A}$ (4.27). Then, there exists $A, \delta, m$ dependent parameters

- $\beta_{0} \in \mathbb{C}$ with $\mathfrak{R}\left(\beta_{0}\right)>0$ large enough and $\mathfrak{J}\left(\beta_{0}\right)>0$ small enough

- $\widetilde{\gamma}_{0}>0$ small enough

such that uniformly in $\left(\left\{y_{a}\right\}_{1}^{n},\left\{z_{a}\right\}_{1}^{n}\right) \in K_{A}^{n} \times K_{A}^{n},|\gamma| \leq \widetilde{\gamma}_{0}, \beta \in \widetilde{\boldsymbol{U}}_{\beta_{0}}$ and $0 \leq n \leq m$

$$
\mathcal{G}_{n ; \gamma}^{(\beta)}\left(\begin{array}{l}
\left\{y_{a}\right\}_{1}^{n} \\
\left\{z_{a}\right\}_{1}^{n}
\end{array}\right)=\mathscr{G}_{\gamma ; A}^{(\beta)}\left[H\left(\cdot \mid \begin{array}{c}
\left\{y_{a}\right\}_{1}^{n} \\
\left\{z_{a}\right\}_{1}^{n}
\end{array}\right)\right] \text { with } H\left(\lambda \mid \begin{array}{c}
\left\{y_{a}\right\}_{1}^{n} \\
\left\{z_{a}\right\}_{1}^{n}
\end{array}\right)=\sum_{a=1}^{n} \frac{1}{\lambda-y_{a}}-\frac{1}{\lambda-z_{a}} .
$$

The functional $\mathscr{G}_{\gamma ; A}^{(\beta)}$ acts on a bounded loop $\mathscr{C}\left(K_{A}\right) \subset U_{\delta}$ around the compact $K_{A}$. The functional $\mathscr{G}_{\gamma ; A}^{(\beta)}[\varpi]$ is a regular functional (cf definition 4.1) of $\varpi$ in respect to the pair $\left(M_{\mathscr{G}_{A}}, \mathscr{C}\left(K_{A}\right)\right)$ where the compact $M_{\mathscr{G}_{A}}$ has its 
boundaries given by $\mathscr{C}_{\text {out }}$ and $\mathscr{C}_{\text {in }}$ as depicted in the rhs of Fig. [1 For all $\varpi \in \mathscr{O}\left(M_{\mathscr{G}_{A}}\right)$ such that $\|\varpi\|_{\mathscr{C}\left(K_{A}\right)} \leq C_{\mathscr{G}_{A}}$, where $C_{\mathscr{G}_{A}}$ is a constant of regularity of the functional $\mathscr{G}_{\gamma ; A}^{(\beta)}$, one has

$$
\begin{aligned}
& \mathscr{G}_{\gamma ; A}^{(\beta)}[\varpi]=\mathrm{e}^{C_{0}\left[\gamma G_{\beta}\right]} \prod_{\epsilon= \pm} \exp \left\{-\oint_{\mathscr{C}\left(K_{A}\right)} \frac{\mathrm{d} z}{2 i \pi} \varpi(z)\left\{2 i \pi C\left[\gamma G_{\beta}\right](z+i \epsilon c)+\ln (z-q+i \epsilon c)\right\}\right\} \\
& \times \exp \left\{-\oint_{\mathscr{C}\left(K_{A}\right)} \frac{\mathrm{d} y \mathrm{~d} z}{(2 i \pi)^{2}} \varpi(y) \varpi(z) \ln (z-y-i c)\right\} \frac{\operatorname{det}_{\mathscr{C}_{A}}\left[I+\gamma \mathscr{V}\left[\gamma G_{\beta}, \gamma G_{\beta}, \varpi\right]\right] \operatorname{det} \mathscr{C}_{A}\left[I+\gamma \overline{\mathscr{V}}\left[\gamma G_{\beta}, \gamma G_{\beta}, \varpi\right]\right]}{\operatorname{det}^{2}[I-K / 2 \pi] \exp \left\{2 i \pi \sum_{\epsilon= \pm} C\left[\gamma G_{\beta}\right](q+i \epsilon c)\right\}} .
\end{aligned}
$$

In the above formula, one should understand $G_{\beta}$ as a one parameter family of functionals of $\varpi$ given by

$$
G_{\beta}(\lambda) \equiv G_{\beta}[\varpi](\lambda)=(i \beta-1 / 2) Z(\lambda)-\phi(\lambda, q)-\oint_{\mathscr{C}\left(K_{A}\right)} \frac{\mathrm{d} z}{2 i \pi} \varpi(z) \phi(\lambda, z) .
$$

In the second line of A.25 there appear Fredholm determinants of integral operators acting on a contour $\mathscr{C}_{A}$. The contour $\mathscr{C}_{A}$ corresponds to a loop around $\mathscr{C}\left(K_{A}\right)$ such that $\mathscr{C}_{A} \subset U_{\delta}$. The kernels read

$\mathscr{V}[v, \rho, \varpi]\left(\omega, \omega^{\prime}\right)=\frac{1}{2 \pi} \frac{\omega-q}{\omega-q+i c} \exp \left\{\oint_{\mathscr{C}\left(K_{A}\right)} \frac{\mathrm{d} z}{2 i \pi} \varpi(z) \ln \left(\frac{\omega-z}{\omega-z+i c}\right)\right\} \cdot \mathrm{e}^{C[2 i \pi v](\omega)-C[2 i \pi v](\omega+i c)} \frac{K\left(\omega-\omega^{\prime}\right)}{\mathrm{e}^{-2 i \pi \rho(\omega)}-1}$

and

$\overline{\mathscr{V}}[v, \rho, \varpi]\left(\omega, \omega^{\prime}\right)=\frac{-1}{2 \pi} \frac{\omega-q}{\omega-q-i c} \exp \left\{\oint_{\mathscr{C}\left(K_{A}\right)} \frac{\mathrm{d} z}{2 i \pi} \varpi(z) \ln \left(\frac{\omega-z}{\omega-z-i c}\right)\right\} \cdot \mathrm{e}^{C[2 i \pi v](\omega)-C[2 i \pi v](\omega-i c)} \frac{K\left(\omega-\omega^{\prime}\right)}{\mathrm{e}^{2 i \pi \rho(\omega)}-1}$

The A, $m$ and $\delta$-dependent parameters $\beta_{0}$ and $\widetilde{\gamma_{0}}$ and the compacts $\mathscr{C}\left(K_{A}\right), M_{\mathscr{G}_{A}}$ are such that the constant of regularity $C_{\mathscr{G}_{A}}$ satisfies to the estimates given in (4.30) and is such that one has

$\forall\|\varpi\|_{\mathscr{C}\left(K_{A}\right)}<C \mathscr{G}_{A} \quad\left\|\widetilde{\gamma}_{0} G_{\beta}[\varpi]\right\|_{U_{\delta}}<1 / 2 \quad$ and $\quad\|H\|_{\mathscr{C}\left(K_{A}\right)}<C_{\mathscr{G}_{A}}$ uniformly in $\left(\left\{y_{a}\right\}_{1}^{n},\left\{z_{a}\right\}_{1}^{n}\right) \in K_{A}^{n} \times K_{A}^{n}$.

Proof-

We first check that $\mathscr{G}_{\gamma ; A}^{(\beta)}$ is a regular functional.

- $G_{\beta}[\varpi]$ is a regular functional as it is linear in $\varpi$ and $\mathscr{C}\left(K_{A}\right)$ is compact.

- the estimates $\left|\mathrm{e}^{x}-\mathrm{e}^{y}\right| \leq \mathrm{e}^{|x|+|y|}|x-y|$, majorations of integrals in terms of sup norm and derivation under the integral sign theorems ensure that all of the exponential pre-factors in $\mathrm{A.25}$ ) are also regular functionals of $\varpi$.

The associated constants of regularity can be taken as large as desired. It thus remains to focus on the Fredholm determinants. For this let us first assume that we are able to pick the contours $\mathscr{C}_{\text {out }}$ in delimiting the boundary of the compact $M_{\mathscr{G}_{A}}$ in such a way that there exists

$$
\beta_{0} \in \mathbb{C} \text { and } \widetilde{\gamma}_{0}>0 \quad \text { such that } \quad \mathrm{e}^{2 i \pi \gamma G_{\beta}[\varpi(*, \boldsymbol{y})](\lambda)}-1 \neq 0 \quad \forall(\lambda, \boldsymbol{y}, \beta, \gamma) \in U_{\delta} \times W_{y} \times \widetilde{\boldsymbol{U}}_{\beta_{0}} \times \mathcal{D}_{0, \widetilde{\gamma}_{0}}
$$


this for any holomorphic function $\varpi(\lambda, \boldsymbol{y})$ on $M_{\mathscr{G}_{A}} \times W_{y}, W_{y} \subset \mathbb{C}^{\ell_{y}}$, that satisfies $\|\varpi\|_{\mathscr{G}\left(K_{A}\right) \times W_{y}}<C_{\mathscr{G}_{A}}$.

If this condition is satisfied, then the integral kernels $\gamma^{\mathscr{V}}\left[\gamma G_{\beta}, \gamma G_{\beta}, \varpi\right]\left(\omega, \omega^{\prime}\right)$ and $\gamma_{\mathscr{V}}\left[\gamma G_{\beta}, \gamma G_{\beta}, \varpi\right]\left(\omega, \omega^{\prime}\right)$ are holomorphic in $\omega, \omega^{\prime}$ belonging to a small neighborhood of $\mathscr{C}_{A}$ and $\boldsymbol{y} \in W_{y}$. The contour $\mathscr{C}_{A}$ being compact, the two integral operators $\gamma \mathcal{V}\left[\gamma G_{\beta}, \gamma G_{\beta}, \varpi\right]$ and $\gamma \overline{\mathcal{V}}\left[\gamma G_{\beta}, \gamma G_{\beta}, \varpi\right]$ are trace class operators that have an analytic dependence on $\boldsymbol{y} \in W_{y}$.

Recall that if $A, B$ are trace class operators $\left(\|\cdot\|_{1}\right.$ stands for the trace class norm) then

$$
|\operatorname{det}[I+A]-\operatorname{det}[I+B]| \leq\|A-B\|_{1} \mathrm{e}^{\|A\|_{1}+\|B\|_{1}+1} .
$$

Also [76], if $A(\boldsymbol{y}), \boldsymbol{y} \in W_{y} \subset \mathbb{C}^{\ell_{y}}$, is an analytic trace class operator then $\operatorname{det}[I+A(\boldsymbol{y})]$ is holomorphic on $W_{y}$ These two properties show that, indeed, in A.25), the two Fredholm determinants of integral operators acting on the contour $\mathscr{C}_{A}$ are regular functionals of $\varpi$.

Hence, it remains to prove the existence of $\widetilde{\gamma}_{0}$ and $\beta_{0}$ such that condition A.18 holds. Given $\varpi(\lambda, \boldsymbol{y}) \in$ $\mathscr{O}\left(M_{\mathscr{G}_{A}} \times W_{y}\right)$, the function $\omega \mapsto \mathrm{e}^{2 i \pi \gamma G_{\beta}[\varpi](\omega)}-1$ has no zeroes provided that

$$
\left|\widetilde{\gamma}_{0} G_{\beta}[\varpi(*, \boldsymbol{y})](\lambda)\right|<1 / 2 \quad \text { and } \quad \mathfrak{J}\left(G_{\beta}[\varpi(*, \boldsymbol{y})](\lambda)\right)>0 \quad \text { uniformly in }(\lambda, \boldsymbol{y}, \beta) \in U_{\delta} \times W_{y} \times \widetilde{\boldsymbol{U}}_{\beta_{0}} .
$$

One has that, for $\beta \in \widetilde{\boldsymbol{U}}_{\beta_{0}}$

$$
\mathfrak{J}\left(G_{\beta}[\varpi](\lambda)\right)>\mathfrak{R}\left(\beta_{0}\right) \inf _{\lambda \in U_{\delta}}[\mathfrak{R}(Z(\lambda))]-\left(\mathfrak{J}\left(\beta_{0}\right)+1 / 2\right)\|\mathfrak{J}(Z)\|_{U_{\delta}}-\|\phi\|_{U_{\delta}^{2}}-\|\varpi\|_{\mathscr{C}\left(K_{A}\right) \times W_{y}} \sup _{\lambda \in U_{\delta}} \oint_{\mathscr{C}\left(K_{A}\right)} \frac{|\mathrm{d} z|}{2 \pi}|\phi(\lambda, z)| .
$$

Hence, $\mathfrak{J}\left(G_{\beta}[\varpi](\lambda)\right)>0$ as soon as $\|\varpi\|_{\mathscr{C}\left(K_{A}\right) \times W_{y}} \leq C_{\mathscr{G}_{A}}$ with

$$
C_{\mathscr{G}_{A}}=\left\{2 \sup _{\lambda \in U_{\delta}} \oint_{\mathscr{C}\left(K_{A}\right)} \frac{|\mathrm{d} z|}{2 \pi}|\phi(\lambda, z)|\right\}^{-1} \cdot\left\{\mathfrak{R}\left(\beta_{0}\right) \inf _{\lambda \in U_{\delta}}[\mathfrak{R}(Z(\lambda))]-\left(\mathfrak{J}\left(\beta_{0}\right)+1 / 2\right)\|\mathfrak{J}(Z)\|_{U_{\delta}}-\|\phi\|_{U_{\delta}^{2}}\right\} .
$$

Here $\mathfrak{R}\left(\beta_{0}\right)>0$ is taken large enough for $C_{\mathscr{G}_{A}}$ as defined above to be positive. Then, if $\|\varpi\|_{\mathscr{C}\left(K_{A}\right) \times W_{y}} \leq C_{\mathscr{G}_{A}}$ with $C_{\mathscr{G}_{A}}$ as given above, one has

$$
\begin{aligned}
\sup _{\substack{\omega \in U_{\delta} \\
\boldsymbol{y} \in W_{y}}}\left|G_{\beta}[\varpi(*, y)](\omega)\right| & \leq\left(10 \mathfrak{R}\left(\beta_{0}\right)+\mathfrak{J}\left(\beta_{0}\right)+1 / 2\right)\|Z\|_{U_{\delta}}+\|\phi\|_{U_{\delta}^{2}}+\|\varpi\|_{\mathscr{C}\left(K_{A}\right)} \sup _{\lambda \in U_{\delta}} \oint_{\mathscr{C}\left(K_{A}\right)} \frac{|\mathrm{d} z|}{2 \pi}|\phi(\lambda, z)| \\
& <\left(11 \mathfrak{R}\left(\beta_{0}\right)+2 \mathfrak{J}\left(\beta_{0}\right)+1\right)\|Z\|_{U_{\delta}} .
\end{aligned}
$$

Hence, if we take $\widetilde{\gamma}_{0}^{-1}=2\left(11 \Re\left(\beta_{0}\right)+2 \mathfrak{J}\left(\beta_{0}\right)+1\right)\|Z\|_{U_{\delta}}$, the condition $\left|\gamma G_{\beta}[\varpi]\right|<1 / 2$ will be satisfied for all $|\gamma| \leq \widetilde{\gamma}_{0}$ and $\beta \in \widetilde{\boldsymbol{U}}_{\beta_{0}}$. It remains to tune $\mathfrak{R}\left(\beta_{0}\right)$ so that conditions

$$
C_{\mathscr{G}_{A}} \cdot \frac{\pi \mathrm{d}\left(\partial M_{\mathscr{G}_{A}}, \mathscr{C}\left(K_{A}\right)\right)}{\left|\partial M_{\mathscr{G}_{A}}\right|+2 \pi \mathrm{d}\left(\partial M_{\mathscr{G}_{A}}, \mathscr{C}\left(K_{A}\right)\right)}>A \quad \text { and } \quad \frac{2 m}{\mathrm{~d}\left(K_{A}, \mathscr{C}\left(K_{A}\right)\right)}<C_{\mathscr{G}_{A}} .
$$

are satisfied.

One can always choose the contours $\mathscr{C}_{\text {out } / \text { in }}$ defining $\partial M_{\mathscr{G}_{A}}$ in such a way that $\mathrm{d}\left(\partial M_{\mathscr{G}_{A}}, \mathscr{C}\left(K_{A}\right)\right)>c$ this uniformly in $\mathrm{A}>0$. These contours can also be chosen such that there exists an $A$-independent constant $c_{1}$ with $\left|\partial M_{\mathscr{G}_{A}}\right|<c_{1} A$. It is also clear that the contour $\mathscr{C}\left(K_{A}\right)$ surrounding the compact $K_{A}$ can be chosen such that $\left|\mathscr{C}\left(K_{A}\right)\right|<c_{2} A$ for some $A$-independent constant $c_{2}$ and also $\mathrm{d}\left(K_{A}, \mathscr{C}\left(K_{A}\right)\right)>c^{\prime}$. It is then enough to take 
$\mathfrak{R}\left(\beta_{0}\right)>c_{\beta_{0}} A^{3}$ with $c_{\beta_{0}}$ being properly tuned in terms of $c, c^{\prime}, c_{1}, c_{2}$ so that conditions (A.23) hold for any $A$ sufficiently large.

Note that the second condition in (A.23) guarantees that the function $H$ as given in (A.13) satisfies $\|H\|_{\mathscr{C}\left(K_{A}\right)}<$ $C_{\mathscr{G}_{A}}$ uniformly in the parameters $\left(\left\{\mu_{p_{a}}\right\}_{1}^{n},\left\{\mu_{h_{a}}\right\}_{1}^{n}\right) \in K_{A}^{n} \times K_{A}^{n}$.

Having proved that $\mathscr{G}_{\gamma ; A}^{(\beta)}$ is a regular functional with a regularity constant $C_{\mathscr{G}_{A}}>0$ sufficiently large, we can evaluate it on $H$. Then, it is readily seen that $G_{\beta}[\varpi](\lambda)$ coincides with the shift function $F_{\beta}$ once upon taking $\varpi=H$ as given in (A.13). All other integrals involving $\varpi=H$ are computed by the residues at $\mu_{p_{a}}$ and $\mu_{h_{a}}$. All calculations done, one recovers the representation (A.8) for the function $\mathcal{G}_{n ; \gamma}^{(\beta)}$. We stress that the parameters $\widetilde{\gamma}_{0}$ and $\beta_{0}$ ensuring the regularity of the functional $\mathscr{G}_{\gamma ; A}^{(\beta)}$ are also such that $\mathcal{G}_{n ; \gamma}^{(\beta)}$ is well defined due to conditions A.20).

\section{Regular functional for $\widehat{\mathcal{G}}_{N ; \gamma}$}

A very similar representation to the one given in the previous lemma exists for the functional $\widehat{\mathcal{G}}_{N ; \gamma}$.

Lemma A.2 Let $m \in \mathbb{N}$ and the strip $U_{\delta}$ be fixed. Let $A>0$ be some constant defining the size of the compact $K_{A}$ (4.27). Then, there exists $A, m$ and $\delta$-dependent constants

- $\beta_{0} \in \mathbb{C}$ with $\mathfrak{R}\left(\beta_{0}\right)>0$ large enough and $\mathfrak{J}\left(\beta_{0}\right)>0$ small enough,

- $\tilde{\gamma}_{0}>0$ small enough,

such that for L large enough and uniformly in $\left(\left\{\mu_{p_{a}}\right\}_{1}^{n},\left\{\mu_{h_{a}}\right\}_{1}^{n}\right) \in K_{A}^{n} \times K_{A}^{n},|\gamma| \leq \widetilde{\gamma}_{0}$ and $0 \leq n \leq m$

$$
\widehat{\mathcal{G}}_{N ; \gamma}\left(\begin{array}{l}
\left\{p_{a}\right\}_{1}^{n} \\
\left\{h_{a}\right\}_{1}^{n}
\end{array}\right)\left[\gamma F_{\beta}, \xi, \xi_{\gamma F_{\beta}}\right]=\widehat{\mathscr{G}}_{\gamma ; A}^{(\beta)}\left[H\left(* \mid \begin{array}{l}
\left\{\mu_{p_{a}}\right\}_{1}^{n} \\
\left\{\mu_{h_{a}}\right\}_{1}^{n}
\end{array}\right)\right] \quad \text { with } \quad H\left(\lambda \mid \begin{array}{c}
\left\{\mu_{p_{a}}\right\}_{1}^{n} \\
\left\{\mu_{h_{a}}\right\}_{1}^{n}
\end{array}\right)=\sum_{a=1}^{n} \frac{1}{\lambda-\mu_{p_{a}}}-\frac{1}{\lambda-\mu_{h_{a}}} .
$$

The functional $\widehat{\mathscr{G}}_{\gamma ; A}^{(\beta)}$ acts on a bounded loop $\mathscr{C}\left(K_{A}\right) \subset U_{\delta}$ around the compact $K_{A}$. The functional $\widehat{\mathscr{G}}_{\gamma ; A}^{(\beta)}[\varpi]$ is a regular functional (cf definition 4.1) of $\varpi$ in respect to the pair $\left(M_{\mathscr{G}_{A}}, \mathscr{C}\left(K_{A}\right)\right)$ where the compact $M_{\mathscr{G}_{A}}$ has its boundaries given by $\mathscr{C}_{\text {out }}$ and $\mathscr{C}_{\text {in }}$ as depicted in the rhs of Fig. 目 For all $\varpi \in \mathscr{O}\left(M_{\mathscr{G}_{A}}\right)$ such that $\|\varpi\|_{\mathscr{C}\left(K_{A}\right)} \leq C_{\mathscr{G}_{A}}$, where $C_{\mathscr{G}_{A}}$ is a constant of regularity of the functional $\widehat{\mathscr{G}}_{\gamma ; A}^{(\beta)}$,

$$
\begin{aligned}
& \widehat{\mathscr{G}}_{\gamma ; A}^{(\beta)}[\varpi]=W_{N}\left[\gamma G_{\beta}\right]\left(\begin{array}{l}
\left\{\lambda_{a}\right\}_{1}^{N} \\
\left\{\mu_{a}\right\}_{1}^{N}
\end{array}\right) \prod_{\epsilon= \pm} \exp \left\{-\oint_{\mathscr{C}\left(K_{A}\right)} \frac{\mathrm{d} z}{2 i \pi} \varpi(z)\left\{-\ln \left(V_{N ; \epsilon}\left[\gamma G_{\beta}\right]\right)(z)+\ln \left(z-\mu_{N+1}+i \epsilon c\right)\right\}\right\} \\
& \times \exp \left\{-\oint_{\mathscr{C}\left(K_{A}\right)} \frac{\mathrm{d} y \mathrm{~d} z}{(2 i \pi)^{2}} \varpi(y) \varpi(z) \ln (z-y-i c)\right\} \frac{\operatorname{det}_{\mathscr{C}_{A}}\left[I+\gamma^{\mathscr{V}_{N}}\left[\gamma G_{\beta}, \gamma G_{\beta}, \varpi\right]\right] \operatorname{det}_{\mathscr{C}_{A}}\left[I+\widehat{\overline{\mathscr{V}}}_{N}\left[\gamma G_{\beta}, \gamma G_{\beta}, \varpi\right]\right]}{\operatorname{det}_{N+1}\left[\Xi^{(\mu)}[\xi]\right] \operatorname{det}_{N}\left[\Xi^{(\lambda)}\left[\xi_{\gamma G_{\beta}}\right]\right] \prod_{\epsilon= \pm} V_{N ; \epsilon}^{-1}\left[\gamma G_{\beta}[\varpi]\right]\left(\mu_{N+1}\right)} .
\end{aligned}
$$

In the above formula, one should understand $G_{\beta}$ as the one-parameters family of regular functionals of $\varpi$ as defined by (A.15). We did not make the functional dependence of $G_{\beta}$ on $\varpi$ explicit in (A.25). The functionals $W_{N}$ and $V_{N ; \epsilon}$ have been defined in (4.6). We have added the $\left[\gamma G_{\beta}\right]$ symbol so as to make it clear that the parameters $\left\{\lambda_{a}\right\}_{1}^{N}$ entering in their definition are functionals of $\gamma G_{\beta}$ through the relation $\lambda_{a}=\xi_{\gamma G_{\beta}}^{-1}(a / L)$.

In the second line of (A.25) there appear Fredholm determinants of integral operators acting on a contour $\mathscr{C}_{A}$. The contour $\mathscr{C}_{A}$ corresponds to a loop around $\mathscr{C}\left(K_{A}\right)$ such that $\mathscr{C}_{A} \subset U_{\delta}$. The kernels read

$$
\widehat{\mathscr{V}}_{N}[v, \rho, \varpi]\left(\omega, \omega^{\prime}\right)=\frac{1}{2 \pi} \frac{\omega-\mu_{N+1}}{\omega-\mu_{N+1}+i c} \exp \left\{\oint_{\mathscr{C}\left(K_{A}\right)} \frac{\mathrm{d} z}{2 i \pi} \varpi(z) \ln \left(\frac{\omega-z}{\omega-z+i c}\right)\right\} \cdot \frac{V_{N ; 1}[v](\omega)}{V_{N ; 0}[v](\omega)} \cdot \frac{K\left(\omega-\omega^{\prime}\right)}{\mathrm{e}^{-2 i \pi \rho(\omega)}-1}
$$


and

$$
\widehat{\overline{\mathscr{V}}}_{N}[v, \rho, \varpi]\left(\omega, \omega^{\prime}\right)=\frac{-1}{2 \pi} \frac{\omega-\mu_{N+1}}{\omega-\mu_{N+1}-i c} \exp \left\{\oint_{\mathscr{C}\left(K_{A}\right)} \frac{\mathrm{d} z}{2 i \pi} \varpi(z) \ln \left(\frac{\omega-z}{\omega-z-i c}\right)\right\} \cdot \frac{V_{N ;-1}[v](\omega)}{V_{N ; 0}[v](\omega)} \cdot \frac{K\left(\omega-\omega^{\prime}\right)}{\mathrm{e}^{2 i \pi \rho(\omega)}-1}
$$

The constant of regularity $C_{\mathscr{G}_{A}}$ satisfies to the estimates already given in (4.30) and is such that

$$
\forall\|\varpi\|_{\mathscr{C}\left(K_{A}\right)}<C_{\mathscr{G}_{A}} \quad\left\|\widetilde{\gamma}_{0} G_{\beta}[\varpi]\right\|_{U_{\delta}}<1 / 2 \quad \text { and } \quad\|H\|_{\mathscr{C}\left(K_{A}\right)}<C_{\mathscr{G}_{A}} \text { uniformly in }\left(\left\{\mu_{p_{a}}\right\}_{1}^{n},\left\{\mu_{h_{a}}\right\}_{1}^{n}\right) \in K_{A}^{n} \times K_{A}^{n} .
$$

\section{Proof-}

The proof is very similar to the one of lemma A.1. Hence, we only specify that for $L$-large enough, and as soon as condition $\left|\gamma G_{\beta}[\varpi](\lambda)\right|<1 / 2$ for all $\lambda \in U_{\delta}$ is satisfied, the parameters $\lambda_{j}$ are seen to be regular functionals of $\varpi$ thanks to their integral representation

$$
\lambda_{j}[\varpi]=\oint_{\mathscr{C}_{q}} \frac{\mathrm{d} z}{2 i \pi} \frac{\xi_{\gamma G_{\beta}[\varpi]}^{\prime}(z)}{\xi_{\gamma G_{\beta}[\varpi]}(z)-j / L} .
$$

All other details are left to the reader.

\section{A.2 Specific values of the functionals $\mathscr{G}_{\gamma ; A}^{(\beta)}$ and $\widehat{\mathscr{G}}_{\gamma ; A}^{(\beta)}$}

In this subsection, we estimate the value of the functional $\mathscr{G}_{\gamma ; A}^{(\beta)}[\varpi]$ for a specific type of function $\varpi$. This result will play a role later on.

Lemma A.3 Let the function $v(\lambda) \equiv v\left(\lambda \mid\left\{z_{k}\right\}_{1}^{n},\left\{y_{k}\right\}_{1}^{n+1}\right)$ be the unique solution to the linear integral equation driven by the resolvent $R$ of the Lieb kernel (ie $(I-K / 2 \pi)(I+R / 2 \pi)=I)$ :

$$
v(\lambda)+\gamma \int_{-q}^{q} \frac{\mathrm{d} \mu}{2 \pi} R(\lambda, \mu) v(\mu)=(i \beta-1 / 2) Z(\lambda)+\sum_{k=1}^{n} \phi\left(\lambda, z_{k}\right)-\sum_{k=1}^{n+1} \phi\left(\lambda, y_{k}\right) .
$$

Let $A>0$ be large enough and such that $\left(\left\{z_{k}\right\}_{1}^{n},\left\{y_{k}\right\}_{1}^{n+1}\right) \in K_{A}^{n} \times K_{A}^{n+1}$. Let $\beta_{0} \in \mathbb{C}$ and $\widetilde{\gamma}_{0}$ be the two numbers associated to the constant $A$ as stated in lemma A.1 Then defining

$$
\varpi(\lambda)=\sum_{a=1}^{n+1} \frac{1}{\lambda-y_{a}}-\frac{1}{\lambda-q}-\sum_{a=1}^{n} \frac{1}{\lambda-z_{a}}-\int_{-q}^{q} \frac{\gamma v(\tau)}{(\lambda-\tau)^{2}} \mathrm{~d} \tau
$$

the below identity holds

$$
\mathscr{G}_{\gamma ; A}^{(\beta)}[\varpi]=-i c \frac{\prod_{a=1}^{n} \prod_{b=1}^{n+1}\left(y_{b}-z_{a}-i c\right)\left(z_{a}-y_{b}-i c\right)}{\prod_{a, b=1}^{n+1}\left(y_{a}-y_{b}-i c\right) \prod_{a, b=1}^{n}\left(z_{a}-z_{b}-i c\right)} \frac{\operatorname{det}_{n}\left[\delta_{k \ell}+\gamma \widehat{V}_{k \ell}[\gamma v]\right] \operatorname{det}_{n}\left[\delta_{k \ell}+\gamma \widehat{\bar{V}}_{k \ell}[\gamma \nu]\right]}{\operatorname{det}^{2}[I-K / 2 \pi]} .
$$

The non-trivial entries of the two determinants are given by (4.8). The auxiliary variables $\left(\left\{z_{k}\right\}_{1}^{n},\left\{y_{k}\right\}_{1}^{n+1}\right)$ on which these entries depend are undercurrent by the set of auxiliary variables on which depends $v$. 


\section{Proof-}

The function $v$ is bounded on the strip $U_{\delta}$. As a consequence, the associated function $\varpi$ A.30) is also bounded by an $A$ independent constant. The estimates (4.30) for the constant of regularity $C_{\mathscr{G}_{A}}$ for the functional $\mathscr{G}_{\gamma ; A}^{(\beta)}$ ensure that there exists $A$ large enough such that $\|\varpi\|_{\mathscr{C}\left(K_{A}\right)}<C_{\mathscr{G}_{A}}$ uniformly in $\left(\left\{z_{k}\right\}_{1}^{n},\left\{y_{k}\right\}_{1}^{n+1}\right) \in K_{A}^{n} \times K_{A}^{n+1}$. Thus, $\mathscr{G}_{\gamma ; A}^{(\beta)}[\varpi]$ is then well definied.

A direct calculation leads to

$$
\exp \left\{\oint_{\mathscr{C}_{A}} \frac{\mathrm{d} z}{2 i \pi} \varpi(z) \ln \left(\frac{\omega-z}{\omega-z \pm i c}\right)\right\}=\frac{\omega-q \pm i c}{\omega-q} \prod_{a=1}^{n+1} \frac{\omega-y_{a}}{\omega-y_{a} \pm i c} \prod_{a=1}^{n} \frac{\omega-z_{a} \pm i c}{\omega-z_{a}} \mathrm{e}^{C[2 i \pi \gamma v](\omega \pm i c)-C[2 i \pi \gamma v](\omega)} \text {. }
$$

By using the linear integral equation satisfied by $v$ and the representation (A.15) we get that

$$
G_{\beta}[\varpi](\lambda)=v(\lambda)
$$

As a consequence, the kernel $\overline{\mathscr{V}}$ and $\mathscr{V}$ simplify

$$
\mathscr{V}\left[\gamma G_{\beta}[\varpi], \gamma G_{\beta}[\varpi], \varpi\right]\left(\omega, \omega^{\prime}\right)=\prod_{a=1}^{n+1} \frac{\omega-y_{a}}{\omega-y_{a}+i c} \prod_{a=1}^{n} \frac{\omega-z_{a}+i c}{\omega-z_{a}} \frac{K\left(\omega-\omega^{\prime}\right)}{2 \pi\left(\mathrm{e}^{-2 i \pi \gamma v(\omega)}-1\right)}
$$

and

$$
\overline{\mathscr{V}}\left[\gamma G_{\beta}[\varpi], \gamma G_{\beta}[\varpi], \varpi\right]\left(\omega, \omega^{\prime}\right)=-\prod_{a=1}^{n+1} \frac{\omega-y_{a}}{\omega-y_{a}-i c} \prod_{a=1}^{n} \frac{\omega-z_{a}-i c}{\omega-z_{a}} \frac{K\left(\omega-\omega^{\prime}\right)}{2 \pi\left(\mathrm{e}^{2 i \pi \gamma v(\omega)}-1\right)}
$$

The associated Fredholm determinants can now be reduced to finite-size determinants by computing the poles at $\omega=z_{a}$ with $a=1, \ldots, n$ (by definition of $\widetilde{\gamma_{0}}$ and $\beta_{0}$, since $|\gamma| \leq \widetilde{\gamma_{0}}$ and $\beta \in \widetilde{\boldsymbol{U}}_{\beta_{0}}$, there are no poles of $\mathrm{e}^{2 i \pi \gamma \gamma(\omega)}-1$ inside of $\mathscr{C}_{A}$ ).

This leads to

$$
\begin{aligned}
\operatorname{det}_{\mathscr{C}_{A}}\left[I+\gamma \mathscr{V}\left[G_{\beta}[\varpi], G_{\beta}[\varpi], \varpi\right]\right] & =\operatorname{det}_{n}\left[\delta_{k \ell}+\gamma \widehat{V}_{k \ell}[\gamma v]\left(\left\{z_{a}\right\}_{1}^{n},\left\{y_{a}\right\}_{1}^{n+1}\right)\right] \\
\operatorname{det}_{\mathscr{C}_{A}}\left[I+\gamma \overline{\mathscr{V}}\left[G_{\beta}[\varpi], G_{\beta}[\varpi], \varpi\right]\right] & =\operatorname{det}_{n}\left[\delta_{k \ell}+\gamma \overline{\bar{V}}_{k \ell}[\gamma v]\left(\left\{z_{a}\right\}_{1}^{n},\left\{y_{a}\right\}_{1}^{n+1}\right)\right] .
\end{aligned}
$$

The claim then follows once upon applying the identity

$$
\begin{aligned}
& -i c \frac{\prod_{a=1}^{n} \prod_{b=1}^{n+1}\left(y_{b}-z_{a}-i c\right)\left(z_{a}-y_{b}-i c\right)}{\prod_{a, b=1}^{n+1}\left(y_{a}-y_{b}-i c\right) \prod_{a, b=1}^{n}\left(z_{a}-z_{b}-i c\right)}=\exp \left\{-\oint_{\mathscr{C}\left(K_{A}\right)} \frac{\mathrm{d} y \mathrm{~d} z}{(2 i \pi)^{2}} \varpi(y) \varpi(z) \ln (z-y-i c)\right\} \\
& \times \mathrm{e}^{C_{0}\left[\gamma G_{\beta}[\varpi]\right]} \mathrm{e}^{-2 i \pi \sum_{\epsilon= \pm} C\left[\gamma G_{\beta}[\varpi]\right](q+i \epsilon c)} \prod_{\epsilon= \pm} \exp \left\{-\oint_{\mathscr{C}\left(K_{A}\right)} \frac{\mathrm{d} z}{2 i \pi} \varpi(z)\left\{2 i \pi C\left[\gamma G_{\beta}[\varpi]\right](z+i \epsilon c)+\ln (z-q+i \epsilon c)\right\}\right\}
\end{aligned}
$$


Lemma A.4 Let $\gamma$ be small enough and L large enough such that $v^{(L)}(\mu)$ is the unique solution to the non-linear integral equation

$$
v^{(L)}(\lambda)=(i \beta-1 / 2) Z(\lambda)-\phi(\lambda, q)+\sum_{a=1}^{N+1} \phi\left(\lambda, \mu_{a}\right)-\sum_{a=1}^{n+1} \phi\left(\lambda, y_{a}\right)-\sum_{\substack{a=1 \\ \neq i_{1}, \ldots, i_{n}}}^{N} \phi\left(\lambda, \tilde{\lambda}_{a}\right)
$$

The parameters $\widetilde{\lambda}_{a}$ appearing above are functional of $v^{(L)}$ through the relation $\xi_{\gamma v^{(L)}}\left(\widetilde{\lambda}_{a}\right)=a / L, \mu_{a}$ are such that $\xi\left(\mu_{a}\right)=a / L$ and the parameters $y_{a} \in U_{\delta / 2}$ are arbitrary. Finally, $L$ is assumed large enough so that all parameters $\left(\left\{\mu_{a}\right\}_{1}^{N+1},\left\{\widetilde{\lambda}_{a}\right\}_{1}^{N},\left\{y_{a}\right\}_{1}^{n+1}\right)$ of the $2 N+2+n$-uple belong to $K_{2 A_{L}}$. Then, given $\beta_{0}$ and $\widetilde{\gamma}_{0}$ as in lemma A.2 one has the identity

$$
\begin{array}{r}
\widehat{\mathscr{G}}_{\gamma ; 2 A_{L}}^{(\beta)}\left[H\left(* \mid \begin{array}{l}
\left\{y_{a}\right\}_{1}^{n+1} \cup\left\{\widetilde{\lambda}_{a}\right\}_{1}^{N} \\
\left\{\widetilde{\lambda}_{i_{a}}\right\}_{1}^{n} \cup\left\{\mu_{a}\right\}_{1}^{N+1}
\end{array}\right)\right]=-i c \frac{\prod_{a=1}^{n} \prod_{b=1}^{n+1}\left(y_{b}-\widetilde{\lambda}_{i_{a}}-i c\right)\left(\widetilde{\lambda}_{i_{a}}-y_{b}-i c\right)}{\prod_{a, b=1}^{n+1}\left(y_{a}-y_{b}-i c\right) \prod_{a, b=1}^{n}\left(\widetilde{\lambda}_{i_{a}}-\widetilde{\lambda}_{i_{b}}-i c\right)} \operatorname{det}_{N+1}^{-1}\left[\Xi^{(\mu)}[\xi]\right] \operatorname{det}_{N}^{-1}\left[\Xi^{(\lambda)}\left[\xi_{\gamma v^{(L)}}\right]\right] \\
\times \operatorname{det}_{n}\left[\delta_{k \ell}+\gamma \widehat{V}_{k \ell}\left[\gamma v^{(L)}\right]\left(\left\{\widetilde{\lambda}_{i_{a}}\right\}_{1}^{n},\left\{y_{a}\right\}_{1}^{n+1}\right)\right] \operatorname{det}_{n}\left[\delta_{k \ell}+\gamma \overline{\bar{V}}_{k \ell}\left[\gamma v^{(L)}\right]\left(\left\{\widetilde{\lambda}_{i_{a}}\right\}_{1}^{n},\left\{y_{a}\right\}_{1}^{n+1}\right)\right] \quad(\mathrm{A} .40)
\end{array}
$$

Proof-

It has been shown in proposition D.3 that for $|\gamma|$ small enough and $L$ large enough the solution $v^{(L)}$ to the non-linear integral equation occurring in the $r h s$ of (A.39) is unique and exists. Moreover this solution is bounded on $U_{\delta}$ by an $L$-independent constant.

As discussed in the proof of lemma A.1 the contour $\mathscr{C}\left(K_{2 A_{L}}\right)$ can always be taken such that, uniformly in $L$, $\mathrm{d}\left(\mathscr{C}\left(K_{2 A_{L}}\right), K_{2 A_{L}}\right)>c^{\prime}>0$ for some constant $c^{\prime}$. Hence, the principal argument $\lambda$ of $H$ is uniformly away from the compact $K_{2 A_{L}}$ where the auxiliary arguments of $H$ are located. As a consequence, it follows from the expression for $H$ and the estimates for the spacing between the parameters $\mu_{a}$ and $\widetilde{\lambda}_{a}$

$$
\mu_{a}-\widetilde{\lambda}_{a}=2 \pi \gamma v^{(L)}\left(\mu_{a}\right) /\left(L p^{\prime}\left(\mu_{a}\right)\right)+\mathrm{O}\left(L^{-2}\right), \quad \text { uniformly in } a=1, \ldots, N
$$

that $\|H\|_{\mathscr{C}\left(K_{2 A_{L}}\right)}$ is bounded by an $L$-independent constant, this uniformly in $L$ large enough. In particular, for $L$ large enough, due to the estimates (4.30) for the constant $C_{\mathscr{G}_{2 A_{L}}}$ of regularity for $\widehat{\mathscr{G}}_{\gamma ; 2 A_{L}}^{(\beta)}$, we get that $\|H\|_{\mathscr{C}\left(K_{A}\right)}<$ $C_{\mathscr{G}_{2 A_{L}}}$. One can thus acts with the functional $\widehat{\mathscr{G}}_{\gamma ; 2 A_{L}}^{(\beta)}$ on $H$. A straightforward residue calculation shows that

$$
G_{\beta}\left[H\left(* \mid \begin{array}{c}
\left\{y_{a}\right\}_{1}^{n+1} \cup\left\{\widetilde{\lambda}_{a}\right\}_{1}^{N} \\
\left\{\widetilde{\lambda}_{i_{a}}\right\}_{1}^{n} \cup\left\{\mu_{a}\right\}_{1}^{N+1}
\end{array}\right)\right]=v^{(L)}(\lambda) .
$$

This means that all the $\lambda_{a}$ appearing in the expression A.25) for the functional $\widehat{\mathscr{G}}_{\gamma ; 2 A_{L}}^{(\beta)}[H]$ coincide with the parameters $\widetilde{\lambda}_{a}$ defined above. The claim of the lemma then follows from straightforward residue computations and multiple cancelations. The Fredholm determinants reduce to finite rank determinants that can be computed by the residues at $\omega=\widetilde{\lambda}_{i_{a}}, a=1, \ldots, n$.

\section{A.3 Leading asymptotic behavior of one particle/one hole form factors}

We now build on the formulae for the leading asymptotic behavior of form factors so as to provide, properly normalized in the size of the model, expressions for the large- $L$ limit of the form factors of the fields between 
the $N$-particle ground state and $N+1$-particle excited states corresponding to one hole at one of the ends of the Fermi zone and one particle either at the other end of the Fermi zone or at the saddle-point $\lambda_{0}$ of the function $u(\lambda)$ given in (2.14). Such thermodynamic limits of properly normalized form factors appear as amplitudes in the large-distance/long-time asymptotic expansion of the reduced density matrix. The explicit expressions that we write down will allow for such an identification. We do stress that all shift functions appearing below are taken at $\beta=0$. The fact that A.46- A.48) are well-defined in this limit follows from proposition A.1

In the following, let $\{\lambda\} \equiv\left\{\lambda_{a}\right\}_{1}^{N}$ stand for the Bethe roots corresponding to the ground state in the $N$-particle sector. Let $\left\{\mu_{\emptyset}^{\emptyset}\right\} \equiv\left\{\mu_{\emptyset}^{\emptyset}\right\}_{1}^{N+1}$ stand for the Bethe roots corresponding to the ground state in the $(N+1)$-particle sector. Taking into account that $F_{\emptyset}^{\emptyset}$ stands for the thermodynamic limit of the corresponding shift function $c f$ (3.5), we define

$$
\left|\mathcal{F}_{\emptyset}^{\emptyset}\right|^{2}=\lim _{N, L \rightarrow+\infty}\left(\frac{L}{2 \pi}\right)^{\left[F_{\emptyset}^{\emptyset}(q)+1\right]^{2}+\left[F_{\emptyset}^{\emptyset}(-q)\right]^{2}}\left|\frac{\left\langle\psi\left(\left\{\mu_{\emptyset}^{\emptyset}\right\}\right)\left|\Phi^{\dagger}(0,0)\right| \psi(\{\lambda\})\right\rangle}{\|\psi(\{\lambda\})\| \cdot\left\|\psi\left(\left\{\mu_{\emptyset}^{\emptyset}\right\}\right)\right\|}\right|^{2} .
$$

Similarly, given the set $\left\{\mu_{q}^{-q}\right\} \equiv\left\{\mu_{q}^{-q}\right\}_{1}^{N+1}$ corresponding to a particle-hole excitation such that $p_{1}=0$ and $h_{1}=$ $N+1$, we denote by $F_{q}^{-q}$ the thermodynamic limit of the corresponding shift function $c f(3.5)$, and define

$$
\left|\mathcal{F}_{q}^{-q}\right|^{2}=\lim _{N, L \rightarrow+\infty}\left(\frac{L}{2 \pi}\right)^{\left[F_{q}^{-q}(q)\right]^{2}+\left[F_{q}^{-q}(-q)-1\right]^{2}}\left|\frac{\left\langle\psi\left(\left\{\mu_{q}^{-q}\right\}\right)\left|\Phi^{\dagger}(0,0)\right| \psi(\{\lambda\})\right\rangle}{\|\psi(\{\lambda\})\| \cdot\left\|\psi\left(\left\{\mu_{q}^{-q}\right\}\right)\right\|}\right|^{2} .
$$

Finally, given the set $\left\{\mu_{q}^{\lambda_{0}}\right\} \equiv\left\{\mu_{q}^{\lambda_{0}}\right\}_{1}^{N+1}$ corresponding to a particle-hole excitation such that $h_{1}=N+1$ and $\mu_{p_{a}}=\lambda_{0}$ we denote by $F_{q}^{\lambda_{0}}$ the thermodynamic limit of the corresponding shift function $c f(3.5)$, and define

$$
\left|\mathcal{F}_{q}^{\lambda_{0}}\right|^{2}=\lim _{N, L \rightarrow+\infty}\left(\frac{L}{2 \pi}\right)^{\left[F_{q}^{\lambda_{0}}(q)\right]^{2}+\left[F_{q}^{\lambda_{0}}(-q)\right]^{2}+1}\left|\frac{\left\langle\psi\left(\left\{\mu_{q}^{\lambda_{0}}\right\}\right)\left|\Phi^{\dagger}(0,0)\right| \psi(\{\lambda\})\right\rangle}{\|\psi(\{\lambda\})\| \cdot\left\|\psi\left(\left\{\mu_{q}^{\lambda_{0}}\right\}\right)\right\|}\right|^{2} .
$$

By using (A.1) and expressions A.3 - A.5 we are lead to

$$
\begin{aligned}
\left|\mathcal{F}_{q}^{\lambda_{0}}\right|^{2} & =\frac{\mathrm{e}^{i \frac{\pi}{4}}}{2 \pi p^{\prime}\left(\lambda_{0}\right)} \mathcal{A}_{0}\left[F_{q}^{\lambda_{0}}\right] \mathcal{B}\left[F_{q}^{\lambda_{0}}, p\right] \mathcal{G}_{1 ; 1}^{(0)}\left(\begin{array}{c}
\lambda_{0} \\
q
\end{array}\right) \exp \left\{i \frac{\pi}{2}\left(\left[F_{q}^{\lambda_{0}}(-q)\right]^{2}-\left[F_{q}^{\lambda_{0}}(q)\right]^{2}\right)\right\} \\
\left|\mathcal{F}_{q}^{-q}\right|^{2} & =\mathcal{A}_{-}\left[F_{q}^{-q}, p\right] \mathcal{B}\left[F_{q}^{-q}, p\right] \mathcal{G}_{1 ; 1}^{(0)}\left(\begin{array}{c}
-q \\
q
\end{array}\right) \exp \left\{i \frac{\pi}{2}\left(\left[F_{q}^{-q}(-q)-1\right]^{2}-\left[F_{q}^{-q}(q)\right]^{2}\right)\right\}
\end{aligned}
$$

and finally

$$
\left|\mathcal{F}_{\emptyset}^{\emptyset}\right|^{2}=\mathcal{A}_{+}\left[F_{\emptyset}^{\emptyset}, p\right] \mathcal{B}\left[F_{\emptyset}^{\emptyset}, p\right] \mathcal{G}_{0 ; 1}^{(0)}\left(\begin{array}{c}
\emptyset \\
\emptyset
\end{array}\right) \exp \left\{i \frac{\pi}{2}\left(\left[F_{\emptyset}^{\emptyset}(-q)\right]^{2}-\left[F_{\emptyset}^{\emptyset}(q)+1\right]^{2}\right)\right\}
$$

The functionals $\mathcal{B}, \mathcal{A}_{ \pm}$and $\mathcal{A}_{0}$ appearing above have been defined in (4.46), (4.47) and (4.48).

\section{B The generalized free-fermion summation formulae}

In this appendix, we establish summation identities allowing one to recast the form factor expansion of an analogue of the field/conjugated-field two-point function that would appear in a generalized free fermion model in terms 
of a finite-size determinant. The representation we obtain constitutes the very cornerstone for deriving various representations for the correlation functions in the interacting case. In particular, it allows one for an analysis of their asymptotic behavior in the large-distance/long-time regime. We first establish re-summation formulae allowing one to estimate discreet analogs of singular integrals. This will open the way for obtaining Fredholm determinant like representations out of the form factor based expansions.

\section{B.1 Computation of singular sums}

Let $\xi$ stand for the thermodynamic limit of the counting function (2.6) and $E_{-}$be a non-vanishing and holomorphic function in some open neighborhood $U_{\delta}$ of $\mathbb{R}$ such that $\mathfrak{R}\left(\ln E_{-}^{-2}\right)$ has, at most, polynomial growth, ie

$\left|\mathfrak{R}\left[\ln E_{-}^{-2}(\lambda)-i C_{1} \lambda^{k}\right]\right| \leq C_{2}\left|\mathfrak{R}\left(i \lambda^{k-1}\right)\right|+C_{3}, \quad$ for some $C_{1}, C_{2}, C_{3} \in \mathbb{R}^{+} \quad$ and $k>1 \quad$ uniformly in $\lambda \in U_{\delta}$.

The neighborhood $U_{\delta}$ is always taken such that $\xi$ is a biholomorphism on $U_{\delta}$

In the following, we study the below singular sums over the set $\left\{\mu_{a}\right\}$ :

$$
\mathcal{S}_{r}^{(L)}\left[E_{-}^{-2}\right](\lambda)=\sum_{a \in \mathcal{B}_{L}} \frac{E_{-}^{-2}\left(\mu_{a}\right)}{2 \pi L \xi^{\prime}\left(\mu_{a}\right)\left(\mu_{a}-\lambda\right)^{r}} \quad \text { with } \mu_{a} \text { being the unique solution to } \xi\left(\mu_{a}\right)=a / L \text {. }
$$

The summation runs through the set $\mathcal{B}_{L}=\left\{a \in \mathbb{Z}:-w_{L} \leq a \leq w_{L}\right\}$ where $w_{L}$ is some $L$-dependent sequence in $\mathbb{N}$ such that $L=\mathrm{o}\left(w_{L}\right)$ and $\left(w_{L} \cdot L^{-1}\right)^{k-1}=\mathrm{o}(L)$.

Proposition B.1 Let $\mathcal{N}_{q}$ be a compact neighborhood of $[-q ; q]$ lying in $U_{\delta}$, then under the above assumptions and provided that $L$ is large enough, one has, uniformly in $\lambda \in \mathcal{N}_{q}$

$$
\begin{aligned}
& \mathcal{S}_{0}^{(L)}\left[E_{-}^{-2}\right](\lambda)=\int_{\mathscr{C} b k ; L} \frac{\mathrm{d} \mu}{2 \pi} E_{-}^{-2}(\mu)+I_{0}^{(L)}\left[E_{-}^{-2}\right](\lambda) \\
& \mathcal{S}_{1}^{(L)}\left[E_{-}^{-2}\right](\lambda)=\int_{\mathscr{C} b k ; L} \frac{\mathrm{d} \mu}{2 \pi} \frac{E_{-}^{-2}(\mu)}{\mu-\lambda}-i \frac{E_{-}^{-2}(\lambda)}{\mathrm{e}^{2 i \pi L \xi(\lambda)}-1}+I_{1}^{(L)}\left[E_{-}^{-2}\right](\lambda) \\
& \mathcal{S}_{2}^{(L)}\left[E_{-}^{-2}\right](\lambda)=\frac{\partial}{\partial \lambda} \int_{\mathscr{C} b k ; L} \frac{\mathrm{d} \mu}{2 \pi} \frac{E_{-}^{-2}(\mu)}{\mu-\lambda}-i \frac{\partial_{\lambda}\left[E_{-}^{-2}(\lambda)\right]}{\mathrm{e}^{2 i \pi L \xi(\lambda)}-1}+\pi \frac{E_{-}^{-2}(\lambda) L \xi^{\prime}(\lambda)}{2 \sin ^{2}[\pi L \xi(\lambda)]}+I_{2}^{(L)}\left[E_{-}^{-2}\right](\lambda) .
\end{aligned}
$$

The integration goes along the curve $\mathscr{C}_{b k ; L}$ depicted on Fig. (4) Also, given $r \in \mathbb{N}$,

$$
I_{r}^{(L)}\left[E_{-}^{-2}\right](\lambda)=\int_{\mathscr{C} \uparrow: L} \frac{\mathrm{d} z}{2 \pi} \frac{E_{-}^{-2}(z)}{(z-\lambda)^{r}} \frac{1}{1-\mathrm{e}^{-2 i \pi L \xi(z)}}+\int_{\mathscr{C}_{1 ; L}} \frac{\mathrm{d} z}{2 \pi} \frac{E_{-}^{-2}(z)}{(z-\lambda)^{r}} \frac{1}{\mathrm{e}^{2 i \pi L \xi(z)}-1}+\int_{\mathscr{C}_{b d L}} \frac{\mathrm{d} z}{2 \pi} \frac{E_{-}^{-2}(z)}{(z-\lambda)^{r}} .
$$

The contours $\mathscr{C}_{\uparrow \downarrow \downarrow L L}$ are depicted in Fig. 3 whereas $\mathscr{C}_{b d ; L}$ is depicted on Fig. 4 .

The functionals $I_{r}^{(L)}\left[E_{-}^{-2}\right](\lambda)$ are such that $I_{r}^{(L)}\left[E_{-}^{-2}\right](\lambda)=\mathrm{O}\left(\left(L / w_{L}\right)^{k+r-1}\right)$, uniformly in $\lambda \in \mathcal{N}_{q}$.

Proof- 

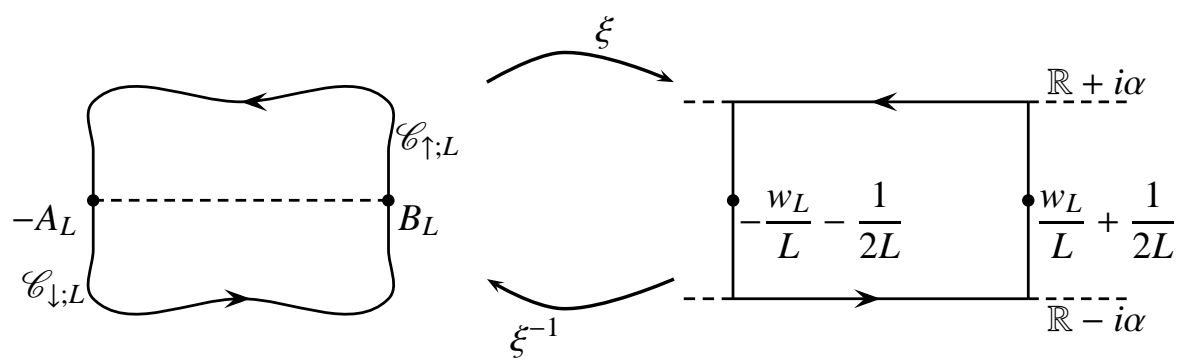

Figure 3: Contour $\mathscr{C}_{\uparrow ; L} \cup \mathscr{C}_{\downarrow ; L}$ lying in $U_{\delta}$.
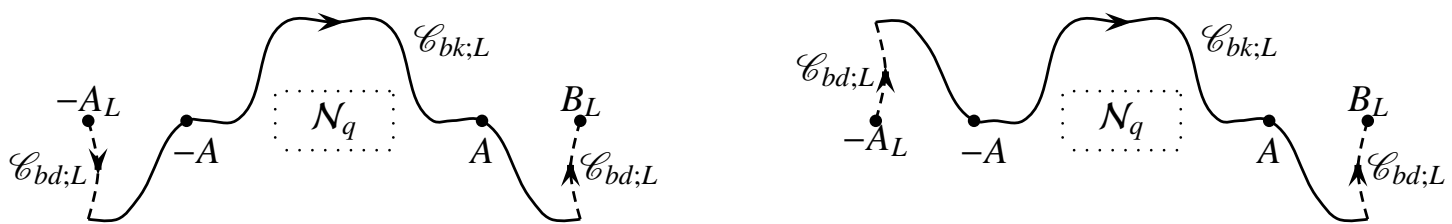

Figure 4: Contours $\mathscr{C}_{b k: L}$ (solid lines) and $\mathscr{C}_{b d ; L}$ (dashed lines) in the case of $k$ odd (lhs) and $k$ even (rhs) in the case $C_{1}<0$. The dotted lines $\mathscr{C}_{b d ; L}$ are pre-images of the segments $\left[\epsilon_{v} w_{L}+\epsilon_{v} 1 / 2 ; \epsilon_{v} w_{L}+\epsilon_{v} 1 / 2+i \epsilon^{\prime} \alpha\right]$, with $v \in\{1, \mathrm{r}\}$ and $\epsilon_{1}=-1$ and $\epsilon_{r}=1$. The sign of $\epsilon^{\prime}$ depends on the left or right boundary, the parity of $k$ and the sign of $C_{1}$.

Let $\mathcal{N}_{q}$ be a compact neighborhood of $[-q ; q]$ in $U_{\delta}$. Then, for $L$ large enough it is contained inside of the contour $\mathscr{C}_{\uparrow ; L} \cup \mathscr{C}_{\downarrow ; L}$ as depicted in Fig. 3], and thus

$$
\begin{aligned}
\mathcal{S}_{1}^{(L)}\left[E_{-}^{-2}\right](\lambda) & =-\frac{i E_{-}^{-2}(\lambda)}{\mathrm{e}^{2 i \pi L \xi(\lambda)}-1}+\int_{\mathscr{C}_{\uparrow ; L} \cup \mathscr{C}_{j ; L}} \frac{E_{-}^{-2}(z)}{2 \pi(z-\lambda)} \cdot \frac{1}{\mathrm{e}^{2 i \pi L \xi(z)}-1} \mathrm{~d} z \\
& =\int_{\mathscr{C}_{j ; L}} \frac{E_{-}^{-2}(z)}{2 \pi(z-\lambda)} \frac{1}{\mathrm{e}^{2 i \pi L \xi(z)}-1} \mathrm{~d} z+\int_{\mathscr{C}_{\uparrow ; L}} \frac{E_{-}^{-2}(z)}{2 \pi(z-\lambda)}\left\{\frac{\mathrm{e}^{2 i \pi L \xi(z)}}{\mathrm{e}^{2 i \pi L \xi(z)}-1}-1\right\} \mathrm{d} z-\frac{i E_{-}^{-2}(\lambda)}{\mathrm{e}^{2 i \pi L \xi(\lambda)}-1} \\
& =\int_{\mathscr{C}_{b k: L}} \frac{\mathrm{d} \mu}{2 \pi} \frac{E_{-}^{-2}(\mu)}{\mu-\lambda}-i \frac{E_{-}^{-2}(\lambda)}{\mathrm{e}^{2 i \pi L \xi(\lambda)}-1}+I_{1}^{(L)}\left[E_{-}^{-2}\right](\lambda) .
\end{aligned}
$$

In order to obtain the last line, we have deformed the contour $\mathscr{C}_{\uparrow ; L}$ into the contour $\mathscr{C}_{b k ; L} \cup \mathscr{C}_{b d ; L}$ as depicted in Fig. 4. The endpoints $\pm A$ are chosen large (in order to include $\mathcal{N}_{q}$ ) but fixed, in the sense that $L$ independent.

The representation for $\mathcal{S}_{2}^{(L)}\left[E_{-}^{-2}\right](\lambda)$ follows by differentiation. The computations for $\mathcal{S}_{0}^{(L)}\left[E_{-}^{-2}\right](\lambda)$ are carried out similarly with the sole difference that there is no pole at $z=\lambda$.

In now remains to prove the statement relative to the asymptotic behavior in $L$ of the functionals $I_{r}^{(L)}\left[E_{-}^{-2}\right]$. The main difficulty is that the function $E_{-}(\lambda)$ might have an exponential increase when $\lambda$ belongs to the upper or lower half-plane. We establish the claimed estimates for the $\mathscr{C}_{\uparrow ; L}$-part of the contour. This can be done similarly for $\mathscr{C}_{\downarrow ; L}$, and we leave these details to the reader. 
We first perform the change of variables $7 z=\xi^{-1}(s)$ and set $u_{L}=w_{L} / L+1 / 2 L$. The contour of integration is then mapped to the contour depicted on the rhs of Fig. 3. We stress that the parameter $\alpha>0$ is chosen in such a way that $\mathscr{C}_{\uparrow ; L} \cup \mathscr{C}_{\downarrow ; L}$ lies in $U_{\delta}$. The aforementioned change of variables leads to

$$
\begin{aligned}
\int_{\mathscr{C} \uparrow: L} \frac{\mathrm{d} z}{2 \pi} \frac{E_{-}^{-2}(z)}{(z-\lambda)^{r}} \cdot \frac{1}{1-\mathrm{e}^{-2 i \pi L \xi(z)}} & =\int_{u_{L}}^{-u_{L}} \frac{\mathrm{d} s}{2 \pi} \frac{E_{-}^{-2}}{\xi^{\prime}} \circ \xi^{-1}(s+i \alpha) \frac{1}{\left[\xi^{-1}(s+i \alpha)-\lambda\right]^{r}}\left\{1-\mathrm{e}^{2 \pi L \alpha} \mathrm{e}^{-2 i \pi s L}\right\}^{-1} \\
+ & \sum_{\epsilon= \pm} \epsilon \int_{0}^{\alpha} \frac{i \mathrm{~d} s}{2 \pi} \frac{E_{-}^{-2}}{\xi^{\prime}} \circ \xi^{-1}\left(i s+\epsilon u_{L}\right) \frac{1}{\left[\xi^{-1}\left(i s+\epsilon u_{L}\right)-\lambda\right]^{r}}\left\{1+\mathrm{e}^{2 \pi s L}\right\}^{-1} .
\end{aligned}
$$

We first establish a bound for the integral over the line $\left[-u_{L} ; u_{L}\right]$. It follows from the integral equation (2.7) satisfied by $p$ that,

$$
p(\lambda)=\lambda \pm \pi D-2 c D / \lambda+\mathrm{O}\left(\lambda^{-2}\right) \quad \text { when } \quad \mathfrak{R}(\lambda) \rightarrow \pm \infty .
$$

Hence, uniformly in $0 \leq \tau \leq \alpha$ and for $s \in \mathbb{R}$ large,

$$
\xi^{-1}(s+i \tau)=\psi_{s}+2 i \pi \tau+\mathrm{O}\left(s^{-2}\right) \quad \text { where } \quad \psi_{s}=2 \pi s-\pi D(1 \pm 1)+\frac{c D}{\pi s} \in \mathbb{R} .
$$

The condition (B.1) implies that there exists constants $C>0, C^{\prime}>0$ such that

$$
\left|\mathfrak{R}\left[\ln E_{-}^{-2}(\lambda)\right]\right| \leq C\left|\mathfrak{R}\left(i \lambda^{k}\right)\right|+C^{\prime}, \quad \text { uniformly in } \lambda \in U_{\delta} .
$$

As a consequence, uniformly in $0 \leq \tau \leq \alpha$,

$$
\begin{aligned}
\left|\mathfrak{R}\left[\ln E_{-}^{-2} \circ \xi^{-1}(s+i \tau)\right]\right| & \leq C\left|\mathfrak{J}\left[\left(\psi_{s}\right)^{k}+2 i \pi k \tau\left(\psi_{s}\right)^{k-1}+\mathrm{O}\left(s^{k-2}\right)\right]\right|+C^{\prime} \\
& \leq C \tau k(2 \pi)^{k}|s|^{k-1}\left|\mathfrak{J}\left(1+\mathrm{O}\left(s^{-1}\right)\right)\right|+C^{\prime}
\end{aligned}
$$

There exists an $s_{0}$ such that $\left|\mathrm{O}\left(s^{-1}\right)\right|<1$ for $|s| \geq s_{0}$, this uniformly in $0 \leq \tau \leq \alpha$. Moreover, for such an $s_{0}$, we define

$$
C^{\prime \prime}=C^{\prime}+\max \left|\mathfrak{R}\left[\ln E_{-}^{-2} \circ \xi^{-1}(s+i \tau)\right]\right|,
$$

with the maximum being taken over $|s| \leq s_{0}$ and $0 \leq \tau \leq \alpha$. Hence, for any $s \in \mathbb{R}$ and $0 \leq \tau \leq \alpha$

$$
\left|\mathfrak{R}\left[\ln E_{-}^{-2} \circ \xi^{-1}(s+i \tau)\right]\right| \leq 2 k C \alpha(2 \pi)^{k}|s|^{k-1}+C^{\prime \prime} .
$$

Therefore, we obtain the estimate

$$
\begin{aligned}
& \left|\int_{u_{L}}^{-u_{L}} \frac{\mathrm{d} s}{2 \pi} \frac{E_{-}^{-2}}{\xi^{\prime}} \circ \xi^{-1}(s+i \alpha) \frac{1}{\left[\xi^{-1}(s+i \alpha)-\lambda\right]^{r}}\left\{1-\mathrm{e}^{2 \pi L \alpha} \mathrm{e}^{-2 i \pi s L}\right\}^{-1}\right| \\
& \leq \sup _{z \in \xi(\mathbb{R}+i \alpha)}\left\{\frac{\mathrm{e}^{C^{\prime \prime}}}{|z-\lambda|^{r} \xi^{\prime}(z)}\right\} \cdot \frac{2 w_{L}+1}{2 \pi L} \cdot \frac{\mathrm{e}^{2 k C \alpha(2 \pi)^{k} u_{L}^{k-1}}}{\mathrm{e}^{2 \pi L \alpha}-1}=\mathrm{O}\left(L^{-\infty}\right),
\end{aligned}
$$

\footnotetext{
${ }^{\dagger}$ we remind that $\xi$ is a biholomorphism on $U_{\delta}$ and that $p^{\prime}>0$ of $\mathbb{R}$.
} 
where we have used that $\left(w_{L} \cdot L^{-1}\right)^{k-1}=\mathrm{o}(L)$. It remains to estimate the integral over the lines $[0 ; \alpha]$ :

$$
\begin{aligned}
&\left|\sum_{\epsilon= \pm} \epsilon \int_{0}^{\alpha} \frac{i \mathrm{~d} s}{2 \pi} \frac{E_{-}^{-2}}{\xi^{\prime}} \circ \xi^{-1}\left(i s+\epsilon u_{L}\right) \frac{1}{\left(\xi^{-1}\left(i s+\epsilon u_{L}\right)-\lambda\right)^{r}}\left\{1+\mathrm{e}^{2 \pi s L}\right\}^{-1}\right| \\
& \sup _{\substack{s \in[0 ; \alpha] \\
\epsilon \in\{ \pm 1\}}}\left\{\frac{1}{\left|\xi^{\prime} \circ \xi^{-1}\left(i s+\epsilon u_{L}\right)\right|\left|\left[\xi^{-1}\left(i s+\epsilon u_{L}\right)-\lambda\right]\right|^{r}}\right\} \times \frac{\mathrm{e}^{C^{\prime \prime}}}{\pi} \int_{0}^{\alpha} \frac{\mathrm{e}^{2 C k(2 \pi)^{k}\left(u_{L}\right)^{k-1} \tau}}{1+\mathrm{e}^{2 \pi L \tau}} \mathrm{d} \tau .
\end{aligned}
$$

By making the change of variables $y=L \tau$ and then applying Lebesgue's dominated convergence theorem, one can convince oneself that the integral in the second line of $(\overline{B .16})$ is a $\mathrm{O}\left(L^{-1}\right)$.

The last class of integrals to consider stems from integrations along $\mathscr{C}_{b d ; L}$. In order to carry the estimates, we need to use the finer condition (B.1). Here we only treat the case of $k$ even and $C_{1}<0$. All other cases are treated very similarly. An analogous reasoning to (B.12) leads, uniformly in $0 \leq \tau \leq \alpha$ to

$$
\mathfrak{R}\left[\ln E_{-}^{-2} \circ \xi^{-1}(s \pm i \tau)\right]=\tau\left(\mp k(2 \pi)^{k} C_{1} s^{k-1}+\mathrm{O}\left(s^{k-2}\right)\right) \quad \text { for } \quad \mp s>0 .
$$

There exists $s_{0}^{\prime}$ such that for $|s| \geq s_{0}^{\prime}$ one has $\left|\mathrm{O}\left(s^{k-2}\right)\right| \leq k(2 \pi)^{k}\left|C_{1} s^{k-1}\right| / 2$. As a consequence, for $|s| \geq s_{0}^{\prime}$ and $\mp s>0$

$$
\mathfrak{R}\left[\ln E_{-}^{-2} \circ \xi^{-1}(s \pm i \tau)\right] \leq-k \frac{(2 \pi)^{k}}{2} \tau\left|C_{1} s^{k-1}\right| \quad \text { uniformly } \quad 0 \leq \tau \leq \alpha .
$$

Therefore

$$
\begin{aligned}
\left|\int_{\mathscr{C}_{b d: L}} \frac{\mathrm{d} \mu}{2 \pi} \frac{E_{-}^{-2}(\mu)}{(\lambda-\mu)^{r}}\right|=\mid \sum_{\epsilon= \pm 1} & \epsilon \int_{0}^{\alpha} \frac{\mathrm{d} \tau}{2 i \pi} \frac{\left(E_{-}^{-2} / \xi^{\prime}\right) \circ \xi^{-1}\left(\epsilon u_{L}-i \epsilon \tau\right)}{\left[\xi^{-1}\left(\epsilon u_{L}-i \epsilon \tau\right)-\lambda\right]^{r}} \mid \\
& \leq 2 \sup _{\substack{\tau \in[0 ; \alpha] \\
\epsilon \in\{ \pm 1\}}}\left|\frac{\left[\xi^{-1}\left(\epsilon u_{L}-i \epsilon \tau\right)-\lambda\right]^{-r}}{\xi^{\prime} \circ \xi^{-1}\left(\epsilon u_{L}-i \epsilon \tau\right)}\right| \int_{0}^{\alpha} \frac{\mathrm{d} \tau}{2 \pi} \mathrm{e}^{-k(2 \pi)^{k} u_{L}^{k-1}\left|C_{1}\right| \frac{\tau}{2}}=\mathrm{O}\left(u_{L}^{1-k-r}\right) .
\end{aligned}
$$

\section{B.2 The generating function: form factor-like representation}

From now on, we assume that the function $E_{-}$takes the form $E_{-}^{-2}(\lambda)=\mathrm{e}^{i x u(\lambda)+g(\lambda)}$ where $u(\lambda)$ is given by (2.14) and $g$ is a bounded holomorphic function on the strip $U_{\delta}$ around $\mathbb{R}(2.15)$. We also assume that $v \in \mathscr{O}\left(U_{\delta}\right)$. We remind that the parameters $\left\{\mu_{a}\right\}_{a \in \mathbb{Z}}$ (resp. $\left\{\lambda_{a}\right\}_{a \in \mathbb{Z}}$ ) are defined as the unique solutions to $L \xi\left(\mu_{a}\right)=a$, (resp. $L \xi_{v}\left(\lambda_{a}\right)=a$ ), where $\xi$ is given by (2.6) and $\xi_{v}(\lambda)=\xi(\lambda)+v(\lambda) / L$. We define the functional $X_{N}\left[v, E_{-}^{2}\right]$ as

$$
X_{N}\left[v, E_{-}^{2}\right]=\sum_{n=0}^{N+1} \sum_{\substack{p_{1}<\cdots<p_{n} \\
p_{k} \in \mathcal{B}_{L}^{\text {ext }}}} \sum_{\substack{h_{1}<\cdots<h_{n} \\
h_{k} \in \mathcal{B}_{L}^{\text {int }}}} \frac{\prod_{a=1}^{N} E_{-}^{2}\left(\lambda_{a}\right)}{\prod_{a=1}^{N+1} E_{-}^{2}\left(\mu_{\ell_{a}}\right)} \widehat{D}_{N}\left(\begin{array}{l}
\left\{p_{a}\right\}_{1}^{n} \\
\left\{h_{a}\right\}_{1}^{n}
\end{array}\right)\left[v, \xi, \xi_{v}\right] .
$$

The functional $\widehat{D}_{N, n}$ has been introduced in (4.4). The sums in (B.20) run through ordered $n-$ uples of integers $p_{1}<\cdots<p_{n}$ belonging to $\mathcal{B}_{L}^{\text {ext }}=\mathcal{B}_{L} \backslash \llbracket 1 ; N+1 \rrbracket$ and through ordered $n-$ uples of integers $h_{1}<\cdots<h_{n}$ 
belonging to $\mathcal{B}_{L}^{\text {in }}=\llbracket 1 ; N+1 \rrbracket$. Finally, $\mathcal{B}_{L}=\left\{j \in \mathbb{Z}:-w_{L} \leq j \leq w_{L}\right\}$ and the sequence $w_{L} \sim L^{\frac{5}{4}}$. In particular, when $L \rightarrow+\infty, w_{L}$ grows much faster then $N$. The integers $\left\{p_{a}\right\}$ and $\left\{h_{a}\right\}$ define the sequence $\ell_{1}<\cdots<\ell_{N+1}$ as explained in (2.3).

The functional $X_{N}\left[v, E_{-}^{2}\right]$ admits two different representations. On the one hand, as written in (B.20), $X_{N}\left[v, E_{-}^{2}\right]$ is closely related to a form factor expansion of certain two-point functions in generalized free-fermion models. On the other hand, after some standard manipulations [60], one can also recast $X_{N}\left[v, E_{-}^{2}\right]$ in terms of a finite-size determinant which goes to a Fredholm minor in the $N, L \rightarrow+\infty$ limit.

We derive this finite-size determinant representation for $X_{N}\left[v, E_{-}^{2}\right]$ below.

Proposition B.2 Under the aforestated assumptions concerning the functions $E_{-}$and $v$, the functional $X_{N}\left[v, E_{-}^{2}\right]$ admits a finite-size determinant representation

$$
X_{N}\left[v, E_{-}^{2}\right]=\left\{\mathcal{S}_{0}^{(L)}\left[E_{-}^{-2}\right]+\frac{\partial}{\partial \alpha}\right\}_{\mid \alpha=0} \cdot \operatorname{det}_{N}\left[\delta_{k \ell}+\frac{V^{(L)}\left(\lambda_{k}, \lambda_{\ell}\right)}{L \xi_{v}^{\prime}\left(\lambda_{\ell}\right)}+\alpha \frac{P^{(L)}\left(\lambda_{k}, \lambda_{\ell}\right)}{L \xi_{v}^{\prime}\left(\lambda_{\ell}\right)}\right]
$$

where

$$
\begin{aligned}
& V^{(L)}(\lambda, \mu)=4 \frac{\sin [\pi v(\lambda)] \sin [\pi v(\mu)]}{2 i \pi(\lambda-\mu)} E_{-}(\mu) E_{-}(\lambda) \cdot\left\{O^{(L)}\left[v, E_{-}^{-2}\right](\lambda)-O^{(L)}\left[v, E_{-}^{-2}\right](\mu)\right\}, \\
& P^{(L)}(\lambda, \mu)=4 \frac{\sin [\pi v(\lambda)] \sin [\pi v(\mu)]}{2 \pi} E_{-}(\lambda) E_{-}(\mu) \cdot O^{(L)}\left[v, E_{-}^{-2}\right](\lambda) \cdot O^{(L)}\left[v, E_{-}^{-2}\right](\mu) .
\end{aligned}
$$

Also, we have set

$$
O^{(L)}\left[v, E_{-}^{-2}\right](\lambda)=i \int_{\mathscr{C}_{b k} ; L} \frac{\mathrm{d} \mu}{2 \pi} \frac{E_{-}^{-2}(\mu)}{\mu-\lambda}+\frac{E_{-}^{-2}(\lambda)}{\mathrm{e}^{-2 i \pi v(\lambda)}-1}+i I_{1}^{(L)}\left[E_{-}^{-2}\right](\lambda) .
$$

The contour of integration has been depicted on Fig. 5 and $S_{0}^{(L)}$ (resp. $\left.I_{r}^{(L)}\right)$ is given by (B.3) (resp. (B.6)).

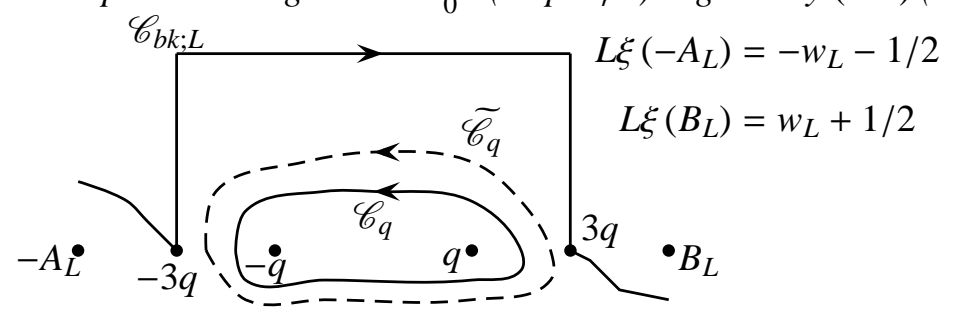

Figure 5: Contour $\mathscr{C}_{b k ; L}$ appearing in the definition of $O^{(L)}\left[v, E_{-}^{-2}\right](\lambda)$, contour $\mathscr{C}_{q}$ (solid line) and contour $\widetilde{\mathscr{C}}_{q}$ (dashed line). The contour $\mathscr{C}_{b k ; L}$ is such that, for $|\mathfrak{R} \lambda| \geq 4 q$, it stays uniformly away from the real axis.

Proof - We first recast the sum over the integers $\left\{p_{a}\right\}$ and $\left\{h_{a}\right\}$ corresponding to particle-hole like excitations into the equivalent sum over all possible choices of integers $\ell_{a}: \ell_{1}<\cdots<\ell_{N+1}$ with $\ell_{a} \in \mathcal{B}_{L}=\mathcal{B}_{L}^{\text {int }} \cup \mathcal{B}_{L}^{\text {ext }}$, cf (2.3). As all the sums are finite, there is no problem in permuting the orders of summation. Therefore,

$$
X_{N}\left[v, E_{-}^{2}\right]=\sum_{\substack{\ell_{1}<\cdots<\ell_{N+1} \\
\ell_{a} \in \mathcal{B}_{L}}} \frac{\prod_{a=1}^{N} E_{-}^{2}\left(\lambda_{a}\right)}{\prod_{a=1}^{N+1} E_{-}^{2}\left(\mu_{\ell_{a}}\right)} \cdot \widehat{D}_{N}\left(\begin{array}{l}
\left\{p_{a}\right\}_{1}^{n} \\
\left\{h_{a}\right\}_{1}^{n}
\end{array}\right)\left[v, \xi, \xi_{v}\right] .
$$


The determinant entering in the definition of $X_{N}\left[v, E_{-}^{2}\right]$ can be represented as

$\prod_{a=1}^{N} \frac{\mu_{a}-\mu_{N+1}}{\lambda_{a}-\mu_{N+1}} \cdot \operatorname{det}_{N}\left[\frac{1}{\mu_{a}-\lambda_{b}}\right]=\operatorname{det}_{N+1}\left[\frac{\left(1-\delta_{b, N+1}\right)}{\mu_{a}-\lambda_{b}}+\delta_{b, N+1}\right]=\left(1+\frac{\partial}{\partial \alpha}\right)_{\mid \alpha=0} \operatorname{det}_{N}\left[\frac{1}{\mu_{a}-\lambda_{b}}-\frac{\alpha}{\mu_{N+1}-\lambda_{b}}\right]$.

There we have used that for any polynomial $Q$ of degree 1 , one has $Q(1)=Q(0)+Q^{\prime}(0)$.

It follows from the above representation that the summand in $(\mathrm{B} .25)$ is a symmetric function of the $N+1$ summation variables $\mu_{\ell_{a}}$ that is moreover vanishing whenever $\ell_{k}=\ell_{a}, k \neq a$. Therefore, we can replace the summation over the fundamental simplex $\ell_{1}<\cdots<\ell_{N+1}$ in the $(N+1)^{\text {th }}$ power Cartesian product $\mathcal{B}_{L}^{N+1}$ by a summation over the whole space $\mathcal{B}_{L}^{N+1}$, provided that we divide the result by $(N+1)$ !. Once that the summation domain is symmetric, we can invoke the antisymmetry of the determinant so as to replace one of the Cauchy determinants by $(N+1)$ ! times the product of its diagonal entries. This last operation produces a separation of variables [60]. Eventually, the result can be recast in the form of a single $N \times N$ determinant:

$$
X_{N}\left[v, E_{-}^{2}\right]=\prod_{a=1}^{N} \frac{4 \sin ^{2}\left[\pi v\left(\lambda_{a}\right)\right]}{\widehat{\xi}_{v}^{\prime}\left(\lambda_{a}\right)} \cdot \sum_{n \in \mathcal{B}_{L}} \frac{E_{-}^{-2}\left(\mu_{n}\right)}{2 \pi L \xi^{\prime}\left(\mu_{n}\right)}\left(1+\frac{\partial}{\partial \alpha}\right)_{\mid \alpha=0} \operatorname{det}_{N}\left[M_{j k}+\alpha \widetilde{P}_{j k}\left(\mu_{n}\right)\right],
$$

with

$$
M_{k \ell}=\delta_{k, \ell} \frac{E_{-}^{2}\left(\lambda_{\ell}\right)}{2 \pi L} \mathcal{S}_{2}^{(L)}\left[E_{-}^{-2}\right]\left(\lambda_{\ell}\right)+\left(1-\delta_{k, \ell}\right) \frac{E_{-}^{2}\left(\lambda_{\ell}\right)}{2 \pi L\left(\lambda_{k}-\lambda_{\ell}\right)}\left\{\mathcal{S}_{1}^{(L)}\left[E_{-}^{-2}\right]\left(\lambda_{k}\right)-\mathcal{S}_{1}^{(L)}\left[E_{-}^{-2}\right]\left(\lambda_{\ell}\right)\right\},
$$

$\mathcal{S}_{r}^{(L)}$ being given by $(\overline{\mathrm{B} .2}),(\overline{\mathrm{B} .3})-(\overline{\mathrm{B} .5})$ and $\widetilde{P}_{j k}\left(\mu_{n}\right)$ being a $\mu_{n}$-dependent rank 1 matrix:

$$
\widetilde{P}_{j k}\left(\mu_{n}\right)=-\frac{E_{-}^{2}\left(\lambda_{k}\right)}{\left(\mu_{n}-\lambda_{j}\right)} \cdot \frac{\mathcal{S}_{1}^{(L)}\left[E_{-}^{-2}\right]\left(\lambda_{k}\right)}{2 \pi L} .
$$

Using the fact that $\widetilde{P}_{j k}\left(\mu_{n}\right)$ is a rank one matrix that contains all the dependence of the determinant on the summation variable $\mu_{n}$, it is readily seen that

$$
\sum_{n \in \mathcal{B}_{L}} \frac{E_{-}^{-2}\left(\mu_{n}\right)}{2 \pi L \xi^{\prime}\left(\mu_{n}\right)}\left(1+\frac{\partial}{\partial \alpha}\right)_{\mid \alpha=0} \cdot \operatorname{det}_{N}\left[M_{j k}+\alpha \widetilde{P}_{j k}\left(\mu_{n}\right)\right]=\left[\mathcal{S}_{0}^{(L)}\left[E_{-}^{-2}\right]+\frac{\partial}{\partial \alpha}\right]_{\mid \alpha=0} \cdot \operatorname{det}_{N}\left[M_{j k}+\alpha P_{j k}\right]
$$

where

$$
P_{j k}=-\frac{E_{-}^{2}\left(\lambda_{k}\right)}{2 \pi L} \mathcal{S}_{1}^{(L)}\left[E_{-}^{-2}\right]\left(\lambda_{k}\right) \cdot \mathcal{S}_{1}^{(L)}\left[E_{-}^{-2}\right]\left(\lambda_{j}\right) .
$$

Applying (B.4), (B.5) and then using that $L \xi\left(\lambda_{k}\right)=L \xi_{v}\left(\lambda_{k}\right)-v\left(\lambda_{k}\right)=k-v\left(\lambda_{k}\right)$, we obtain that

$$
M_{k \ell} \frac{E_{-}\left(\lambda_{k}\right)}{E_{-}\left(\lambda_{\ell}\right)}=\delta_{k \ell} \frac{\xi_{v}^{\prime}\left(\lambda_{\ell}\right)}{4 \sin ^{2}\left[\pi v\left(\lambda_{\ell}\right)\right]}+E_{-}\left(\lambda_{\ell}\right) E_{-}\left(\lambda_{k}\right) \frac{O^{(L)}\left[v, E_{-}^{-2}\right]\left(\lambda_{k}\right)-O^{(L)}\left[v, E_{-}^{-2}\right]\left(\lambda_{\ell}\right)}{2 i \pi L\left(\lambda_{k}-\lambda_{\ell}\right)},
$$

where $O^{(L)}\left[v, E_{-}^{-2}\right]$ is given by $(\mathrm{B} .24)$. Note that we have slightly deformed the form of the contours $\mathscr{C}_{b k ; L}$ in respect to Fig. 4. Very similarly, we find

$$
P_{j k} \frac{E_{-}\left(\lambda_{j}\right)}{E_{-}\left(\lambda_{k}\right)}=\frac{E_{-}\left(\lambda_{j}\right) E_{-}\left(\lambda_{k}\right)}{2 \pi L} O^{(L)}\left[v, E_{-}^{-2}\right]\left(\lambda_{k}\right) O^{(L)}\left[v, E_{-}^{-2}\right]\left(\lambda_{j}\right)=\frac{P^{(L)}\left(\lambda_{j}, \lambda_{k}\right)}{4 L \sin \pi v\left(\lambda_{j}\right) \sin \pi v\left(\lambda_{k}\right)} .
$$

where $P^{(L)}\left(\lambda_{j}, \lambda_{k}\right)$ is given by $(\underline{B .23})$. It then remains to factor out the pre-factors from the determinant. 


\section{B.3 Thermodynamic limit of $X_{N}\left[v, E_{-}^{-2}\right]$}

Proposition B.3 The thermodynamic limit of $X_{N}\left[v, E_{-}^{2}\right]$ is well defined and can be expressed in terms of a Fredholm determinant minor. Namely, $X_{N}\left[v, E_{-}^{2}\right] \underset{N / L \rightarrow D}{\longrightarrow} X_{\mathscr{C}_{E}^{(\infty)}}\left[v, E_{-}^{2}\right]$ with

$$
X_{\mathscr{C}_{E}^{(\infty)}}\left[v, E_{-}^{2}\right]=\left(\mathcal{S}_{\mathscr{C}_{E}^{(\infty)}}\left[E_{-}^{-2}\right]+2 \int_{-q}^{q} \frac{\mathrm{d} \lambda}{\pi} \sin ^{2}[\pi v(\lambda)] F_{+}(\lambda) E_{-}(\lambda) O_{\mathscr{C}_{E}^{(\infty)}}\left[v, E_{-}^{-2}\right](\lambda)\right) \cdot \operatorname{det}[I+V]\left[v, E_{-}^{-2}\right] .
$$

Here $I+V$ is an integral operator on $[-q ; q]$ acting on $L^{2}([-q ; q])$ with a kernel

$$
V(\lambda, \mu)=4 \frac{\sin [\pi v(\lambda)] \sin [\pi v(\mu)]}{2 i \pi(\lambda-\mu)} E_{-}(\lambda) E_{-}(\mu) \cdot\left\{O_{\mathscr{C}_{E}^{(\infty)}}\left[v, E_{-}^{-2}\right](\lambda)-O_{\mathscr{C}_{E}^{(\infty)}}\left[v, E_{-}^{-2}\right](\mu)\right\}
$$

and the contour $\mathscr{C}_{E}^{(w)}$ dependent functionals $O_{\mathscr{C}_{E}^{(w)}}\left[v, E_{-}^{-2}\right](\lambda)$ and $\mathcal{S}_{\mathscr{C}_{E}^{(w)}}\left[E_{-}^{-2}\right]$ are given by

$$
O_{\mathscr{C}_{E}^{(w)}}\left[v, E_{-}^{-2}\right](\lambda)=i \int_{\mathscr{C}_{E}^{(w)}} \frac{\mathrm{d} \mu}{2 \pi} \frac{E_{-}^{-2}(\mu)}{\mu-\lambda}+\frac{E_{-}^{-2}(\lambda)}{\mathrm{e}^{-2 i \pi v(\lambda)}-1} \quad \text { and } \quad \mathcal{S}_{\mathscr{C}_{E}^{(w)}}\left[E_{-}^{-2}\right]=\int_{\mathscr{C}_{E}^{(w)}} \frac{\mathrm{d} \lambda}{2 \pi} E_{-}^{-2}(\lambda) .
$$

$F_{+}(\lambda)$ is the unique solution to the integral equation ${ }^{\dagger}$

$$
\sin [\pi v(\lambda)] F_{+}(\lambda)+\int_{-q}^{q} V(\lambda, \mu) \sin [\pi v(\mu)] F_{+}(\mu) \mathrm{d} \mu=\sin [\pi v(\lambda)] E_{-}(\lambda) O_{\mathscr{C}_{E}^{(\infty)}}\left[v, E_{-}^{-2}\right](\lambda) .
$$

Also, $\mathscr{C}_{E}^{(w)}=\mathscr{C}_{E}^{(\infty)} \cap\{z \in \mathbb{C}:|\mathfrak{R}(z)| \leq w\}$ and $\mathscr{C}_{E}^{(\infty)}$ have been depicted on Fig. 2

This representation can be seen as a generalization of the results obtained in [60]. Also, the contour $\mathscr{C}_{E}^{(\infty)}$ can be thought of as the $L \rightarrow+\infty$ limit of the contour $\mathscr{C}_{b k: L}$.

Proof-

It is a direct consequence of the estimates obtained in appendix B.1 for $I_{r}^{(L)}\left[E_{-}^{-2}\right]$ together with the fact that $\operatorname{det}_{N}\left[\delta_{k \ell}+\mathrm{o}\left(L^{-1}\right)\right] \rightarrow 1$ in the case of remainders $\mathrm{o}\left(L^{-1}\right)$ that are uniform in the entries, that

$$
X_{N}\left[v, E_{-}^{2}\right]_{N / L \rightarrow D}^{\longrightarrow}\left(\mathcal{S}_{\mathscr{C}_{E}^{(\infty)}}\left[E_{-}^{-2}\right]+\frac{\partial}{\partial \alpha}\right)_{\mid \alpha=0} \cdot \operatorname{det}[I+V+\alpha P]
$$

with $I+V+\alpha P$ acting on $[-q ; q]$ and

$$
P(\lambda, \mu)=\frac{2}{\pi} \sin [\pi v(\lambda)] \sin [\pi v(\mu)] E_{-}(\lambda) E_{-}(\mu) O_{\mathscr{C}_{E}^{(\infty)}}\left[v, E_{-}^{-2}\right](\lambda) O_{\mathscr{C}_{E}^{(\infty)}}\left[v, E_{-}^{-2}\right](\mu) .
$$

Note that there is no problem with the integration over an infinite contour $\mathscr{C}_{E}^{(\infty)}$ in $O_{\mathscr{C}_{E}^{(\infty)}}\left[v, E_{-}^{-2}\right](\mu)$ and $\mathcal{S}_{\mathscr{C}_{E}^{(\infty)}}\left[E_{-}^{-2}\right]$ in as much as $\mathscr{C}_{E}^{(\infty)}$ is built precisely in such a way to ensure the exponential decay of the integrand at infinity.

Using that $P$ is a one dimensional projector, we get that

$$
\operatorname{det}[I+V+\alpha P]=\operatorname{det}[I+V]\left(1+\alpha \int_{-q}^{q}(I+V)^{-1}(\lambda, \mu) P(\mu, \lambda) \mathrm{d} \lambda \mathrm{d} \mu\right) .
$$

It then remains to take the $\alpha$-derivative and use the definition of $F_{+}(\lambda)$.

\footnotetext{
${ }^{\dagger}$ By no means $F_{+}$ought to be confused with the shift function
} 


\section{B.4 An algebraic representation for the Fredholm minor}

Proposition B.4 For L large enough, the finite N Fredholm minor $X_{N}\left[v, E_{-}^{2}\right]$ defined in (B.21) can be represented, through purely algebraic manipulations, as the below finite sum:

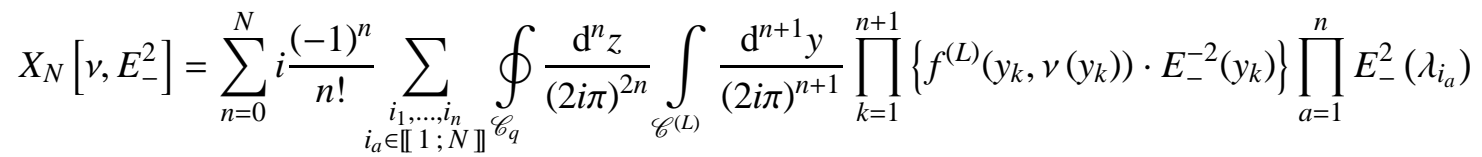

$$
\begin{aligned}
& \times \prod_{k=1}^{n} \frac{y_{n+1}-z_{k}}{\left(y_{n+1}-\lambda_{i_{k}}\right)\left(y_{k}-z_{k}\right)} \cdot \operatorname{det}_{n}\left[\frac{1}{z_{a}-\lambda_{i_{b}}}\right] \prod_{k=1}^{n}\left\{\frac{4 \sin ^{2}\left[\pi v\left(\lambda_{i_{k}}\right)\right]}{\left(z_{k}-\lambda_{i_{k}}\right) L \xi_{v}^{\prime}\left(\lambda_{i_{k}}\right)}\right\} .
\end{aligned}
$$

Above, appear two contours, $\mathscr{C}_{q}$ which stands for a small counterclockwise loop around $[-q ; q]$ as depicted on Fig. [5 and $\mathscr{C}^{(L)}=\mathscr{C}_{b k ; L} \cup \mathscr{C}_{\uparrow ; L} \cup \mathscr{C}_{\downarrow ; L} \cup \mathscr{C}_{b d ; L} \cup \widetilde{\mathscr{C}}_{q}$. As shown on Fig. 5 . $\widetilde{\mathscr{C}}_{q}$ stands for a small counterclockwise loop encircling $\mathscr{C}_{q}$. Finally, the function $f^{(L)}(y, v)$ is supported on $\mathscr{C}^{(L)}$ and reads

$$
f^{(L)}(y, v)=\mathbf{1}_{\mathscr{C}_{b k ; L}}(y)+\frac{1}{1-\mathrm{e}^{-2 i \pi L \xi(y)}} \mathbf{1}_{\mathscr{C}_{i ; L}}(y)+\frac{1}{\mathrm{e}^{2 i \pi L \xi(y)}-1} \mathbf{1}_{\mathscr{L}_{1 ; L}}(y)-\frac{1}{\mathrm{e}^{-2 i \pi v}-1} \mathbf{1}_{\widetilde{\mathscr{C}}_{q}}(y)+\mathbf{1}_{\mathscr{C}_{b d ; L}}(y) .
$$

where $\mathbf{1}_{A}$ stands for the indicator function of $A$.

Proof-

The functional $O^{(L)}\left[v, E_{-}^{-2}\right](z)$ as defined in $(\mathrm{B.24})$ is holomorphic in some sufficiently small open neighborhood of $[-q ; q]$. Hence, there exists a small counterclockwise loop $\mathscr{C}_{q}$ around $[-q ; q]$ (cf Fig. 5) such that the kernel $V^{(L)}(\lambda, \mu)$ admits the integral representation

$V^{(L)}(\lambda, \mu)=4 \sin [\pi v(\lambda)] \sin [\pi v(\mu)] E_{-}(\lambda) E_{-}(\mu) \oint_{\mathscr{C}_{q}} \frac{O^{(L)}\left[v, E_{-}^{-2}\right](z)}{(z-\lambda)(z-\mu)} \frac{\mathrm{d} z}{(2 i \pi)^{2}}, \quad$ for $\quad \lambda, \mu \in\left\{\lambda_{1}, \ldots, \lambda_{N}\right\}$.

In (B.43) we have used that $\lambda_{1}, \ldots, \lambda_{N}$ are all inside of $\mathscr{C}_{q}$ for $L$ large enough. We first expand the $N \times N$ determinant appearing in the final expression for $X_{N}\left[v, E_{-}^{2}\right]$ into its discreet Fredholm series:

$$
\operatorname{det}_{N}\left[\delta_{k \ell}+\frac{V^{(L)}\left(\lambda_{k}, \lambda_{\ell}\right)}{L \xi_{v}^{\prime}\left(\lambda_{\ell}\right)}+\alpha \frac{P^{(L)}\left(\lambda_{k}, \lambda_{\ell}\right)}{L \xi_{v}^{\prime}\left(\lambda_{\ell}\right)}\right]=\sum_{n=0}^{N} \sum_{\substack{i_{1}, \ldots, i_{n} \\ i_{a} \in \mathbb{1} ; N \rrbracket}} \frac{\operatorname{det}_{n}\left[V^{(L)}\left(\lambda_{i_{a}}, \lambda_{i_{b}}\right)+\alpha P^{(L)}\left(\lambda_{i_{a}}, \lambda_{i_{b}}\right)\right]}{n ! \prod_{k=1}^{n}\left[L \xi_{v}^{\prime}\left(\lambda_{i_{k}}\right)\right]}
$$

Next, observe that

$$
\begin{aligned}
\operatorname{det}_{n}\left[V^{(L)}\left(\lambda_{i_{a}}, \lambda_{i_{b}}\right)+\alpha P^{(L)}\left(\lambda_{i_{a}}, \lambda_{i_{b}}\right)\right] & =\oint_{\mathscr{C}_{q}} \frac{\mathrm{d}^{n} z}{(2 i \pi)^{2 n}} \prod_{a=1}^{n}\left\{\frac{O^{(L)}\left[v, E_{-}^{-2}\right]\left(z_{a}\right)}{z_{a}-\lambda_{i_{a}}}\right\} \\
& \times \prod_{a=1}^{n}\left\{4 \sin ^{2}\left[\pi v\left(\lambda_{i_{a}}\right)\right] E_{-}^{2}\left(\lambda_{i_{a}}\right)\right\} \times \operatorname{det}_{n+1}\left[\begin{array}{rr}
\left(z_{a}-\lambda_{i_{b}}\right)^{-1} & \alpha \\
-i O^{(L)}\left[v, E_{-}^{-2}\right]\left(\lambda_{i_{b}}\right) & 1
\end{array}\right]
\end{aligned}
$$

It can be readily seen that for any $z$ belonging to the interior of $\widetilde{\mathscr{C}}_{q}$

$$
O^{(L)}\left[v, E_{-}^{-2}\right](z)=\int_{\mathscr{C}(L)} \frac{i \mathrm{~d} y}{2 \pi} \frac{f^{(L)}(y, v(y))}{y-z} E_{-}^{-2}(y) \quad \text { with } \quad \mathscr{C}^{(L)}=\mathscr{C}_{b k ; L} \cup \mathscr{C}_{\uparrow ; L} \cup \mathscr{C}_{\downarrow ; L} \cup \widetilde{\mathscr{C}}_{q} \cup \mathscr{C}_{b d ; L},
$$


and $f^{(L)}$ is as given by $(\underline{B .42})$. Then, using the multilinear structure of a determinant, one gets that

$$
\begin{aligned}
& \left\{\mathcal{S}_{0}^{(L)}\left[E_{-}^{-2}\right]+\frac{\partial}{\partial \alpha}\right\}_{\alpha=0} \cdot \operatorname{det}_{n+1}\left[\begin{array}{cc}
\left(z_{a}-\lambda_{i_{b}}\right)^{-1} & \alpha \\
-i O^{(L)}\left[v, E_{-}^{-2}\right]\left(\lambda_{i_{a}}\right) & 1
\end{array}\right]=\operatorname{det}_{n+1}\left[\begin{array}{cc}
\left(z_{a}-\lambda_{i_{b}}\right)^{-1} & 1 \\
-i O^{(L)}\left[v, E_{-}^{-2}\right]\left(\lambda_{i_{b}}\right) & \mathcal{S}_{0}^{(L)}\left[E_{-}^{-2}\right]
\end{array}\right] \\
& =\int_{\mathscr{C}(L)} \frac{\mathrm{d} y}{2 \pi} E_{-}^{-2}(y) f^{(L)}(y, v(y)) \operatorname{det}_{n+1}\left[\begin{array}{cc}
\left(z_{a}-\lambda_{i_{b}}\right)^{-1} & 1 \\
\left(y-\lambda_{i_{b}}\right)^{-1} & 1
\end{array}\right]=\int_{\mathscr{\mathscr { C }}(L)} \frac{\mathrm{d} y}{2 \pi} E_{-}^{-2}(y) f^{(L)}(y, v(y)) \prod_{k=1}^{n} \frac{z_{k}-y}{\lambda_{i_{k}}-y} \operatorname{det}_{n}\left[\frac{1}{z_{a}-\lambda_{i_{b}}}\right] .
\end{aligned}
$$

The leads to the claim, once upon inserting this representation into the discreet Fredholm series.

\section{B.5 The Natte series for a Fredholm minor}

In this subsection, we recall the form of the Natte series representation for the Fredholm minor (B.34) involved in the representation of form factor sums in generalized free fermionic models. We refer the reader to theorem 2.2 and proposition 7.2 of reference [62] for further details relative to this Natte series expansion.

Let $E_{-}^{2}=\mathrm{e}^{-i x u(\lambda)-g(\lambda)}$ be such that

- $u$ and $g$ are holomorphic in the open neighborhood $U_{\delta / 2}$ of $\mathbb{R}$;

- $u$ has a unique saddle-point $\lambda_{0}$ on the real axis which is of order 1 , ie $u^{\prime \prime}\left(\lambda_{0}\right)<0$;

- the function $v$ is holomorphic in an open neighborhood $\mathcal{N}_{q} \subset U_{\delta / 2}$ of $[-q ; q]$.

Also, let $\mathscr{C}_{E}^{(w)}=\mathscr{C}_{E}^{(\infty)} \cap\{z \in \mathbb{C}:|\mathfrak{R} z|<w\}$. The contours $\mathscr{C}_{E}^{(\infty)}$ and $\mathscr{C}_{E}^{(w)}$ have been depicted in Fig. [2.

For $w>\left|\lambda_{0}\right|+q>0$ and $x$ large enough, the Fredholm minor $X_{\mathscr{C}_{E}^{(w)}}\left[v, E_{-}^{2}\right]$ defined in (B.34) admits the below Natte series representation

$$
\begin{aligned}
& X_{\mathscr{C}_{E}^{(w)}}\left[v, E_{-}^{2}\right]=\frac{\mathcal{B}\left[v, u+i 0^{+}\right]}{x^{\nu^{2}(q)+\nu^{2}(-q)}} \mathrm{e}^{\int^{-q}\left[i x u^{\prime}(\lambda)+g^{\prime}(\lambda)\right] v(\lambda) \mathrm{d} \lambda}\left\{\frac{\mathcal{A}_{0}[v] \mathbf{1}_{1 q ;+\infty}\left[\left(\lambda_{0}\right)\right.}{\sqrt{-2 \pi x u^{\prime \prime}\left(\lambda_{0}\right)}} \mathrm{e}^{i x u\left(\lambda_{0}\right)+g\left(\lambda_{0}\right)}+\frac{\mathcal{A}_{+}\left[v, u+i 0^{+}\right]}{x^{1+2 v(q)}} \mathrm{e}^{i x u(q)+g(q)}\right. \\
& \left.+\frac{\mathcal{A}_{-}[v, u]}{x^{1-2 v(-q)}} \mathrm{e}^{i x u(-q)+g(-q)}+\sum_{n \geq 1} \sum_{\mathcal{K}_{n}} \sum_{\mathcal{E}_{n}\left(\left\{k_{a}\right\}\right)} \int_{\mathscr{C}_{\epsilon_{t}}^{(w)}} H_{n ; x}^{\left(\left\{\epsilon_{t}\right\}\right)}\left(\left\{u\left(z_{t}\right)\right\} ;\left\{z_{t}\right\}\right)[v] \prod_{t \in J_{\{k\}}} \mathrm{e}^{\epsilon_{t} g\left(z_{t}\right)} \frac{\mathrm{d}^{n} z_{t}}{(2 i \pi)^{n}}\right\} .
\end{aligned}
$$

The $+i 0^{+}$regularization of $u$ only matters in the time-like regime (where $\left|\lambda_{0}\right|<q$ ). The functionals $\mathcal{B}, \mathcal{A}_{ \pm}$and $\mathcal{A}_{0}$ are given respectively by (4.46) (4.47) and (4.48). The notations and the structure of the sums appearing in the second line of (B.48) are exactly as explained in theorem 4.1

The Natte series is convergent for $x$ large enough in as much as, for $n$ large enough,

$$
\sum_{\mathcal{K}_{n}} \sum_{\mathcal{E}_{n}\left(\left\{k_{a}\right\}\right)}\left\|H_{n ; x}^{\left(\left\{\epsilon_{t}\right\}\right)}[v] \prod_{t \in J_{\{k\}}} \mathrm{e}^{\epsilon_{t} g}\right\|_{L^{1}\left(\mathscr{C}_{\epsilon_{t}}^{(\infty)}\right)} \leq c_{2}\left(\frac{c_{1}}{x}\right)^{n c_{3}}
$$

There $c_{1}$ and $c_{2}$ are some constants depending on the values taken by $u$, and $g$ in some small neighborhood of the base curve $\mathscr{C}_{E}^{(\infty)}$ and by $v$ on a small neighborhood of $[-q ; q]$, whereas

$c_{3}=\frac{3}{4} \min \left(1 / 2,1-2 \max _{\tau= \pm}|\mathfrak{R}[v(\tau q)]|-\Upsilon_{\epsilon}\right) \quad$ where $\Upsilon_{\epsilon}=2 \sup \{|\mathfrak{R}[v(z)-v(\tau q)]|:|z-\tau q| \leq \epsilon, \tau= \pm\}$. 
Here $\epsilon>0$ is sufficiently small but arbitrary otherwise. We stress that, should these norms change, then so would change the constants $c_{1}, c_{2}$ and $c_{3}$ but the overall structure of the estimates in $x$ would remain.

The Natte series expansion (B.48) has a well defined $w \rightarrow+\infty$ limit: all the concerned integrals are convergent as the functions $H_{n ; x}^{\left(\left\{\epsilon_{t}\right)\right)}$ approach zero exponentially fast in respect to any variable that runs to $\infty$ along $\mathscr{C}_{\epsilon_{t}}^{(\infty)}$. Moreover, this limit does not alter in any way the estimates (B.49) ensuring the convergence of the Natte series (the constants $c_{1}-c_{3}$ are $w$-independent).

We now list several properties of the functions $H_{n, x}^{\left(\left\{\epsilon_{t}\right\}\right)}$ :

i) $H_{n ; x}^{\left(\left\{\epsilon_{t}\right\}\right)}\left(\left\{u\left(z_{t}\right)\right\} ;\left\{z_{t}\right\}\right)[v]$ is a function of $\left\{u\left(z_{t}\right)\right\}$ and $\left\{z_{t}\right\}$. It is also a regular functional of $v$.

ii) $H_{n ; x}^{\left(\left\{\epsilon_{t}\right\}\right)}\left(\left\{u\left(z_{t}\right)\right\} ;\left\{z_{t}\right\}\right)[\gamma v]=\mathrm{O}\left(\gamma^{n}\right)$ and the $\mathrm{O}$ holds in the $\left(L^{1} \cap L^{\infty}\right)\left(\mathscr{C}_{\epsilon_{t}}^{(\infty)}\right)$ sense.

iii) $H_{n ; x}^{\left(\left\{\epsilon_{t}\right\}\right)}$ can be represented as:

$$
H_{n ; x}^{\left(\left\{\epsilon_{t}\right\}\right)}\left(\left\{u\left(z_{t}\right)\right\} ;\left\{z_{t}\right\}\right)[v]=\widetilde{H}_{n ; x}^{\left(\left\{\epsilon_{t}\right\}\right)}\left(\left\{v\left(z_{t}\right)\right\} ;\left\{u\left(z_{t}\right)\right\} ;\left\{z_{t}\right\}\right) \prod_{t \in J_{\{k\}}}\left(\varkappa[v]\left(z_{t}\right)\right)^{-2 \epsilon_{t}} \times \prod_{\substack{t \in J_{\{k\}} \\ \epsilon_{t}=1}}\left(\mathrm{e}^{-2 i \pi v\left(z_{t}\right)}-1\right)^{2} .
$$

$\widetilde{H}_{n ; x}^{\left(\left\{\epsilon_{t}\right\}\right)}$ is a holomorphic function for $|\mathfrak{R}(v)| \leq 1 / 2$ and $\varkappa[v](\lambda)$ has been defined in (4.47).

iv) One has $H_{1 ; x}^{\left(\left\{\epsilon_{t}\right\}\right)}=\mathrm{O}\left(x^{-\infty}\right)$ and for $n \geq 2$

$$
H_{n ; x}^{\left(\left\{\epsilon_{\epsilon}\right\}\right)}=\mathrm{O}\left(x^{-\infty}\right)+\sum_{b=0}^{[n / 2]} \sum_{p=0}^{b} \sum_{m=b-\left[\frac{n}{2}\right]}^{[n / 2]-b}\left(\frac{\mathrm{e}^{i x\left[u\left(\lambda_{0}\right)-u(-q)\right]}}{x^{2 v(-q)}}\right)^{\eta b} \cdot\left(\frac{\mathrm{e}^{i x[u(q)-u(-q)]}}{x^{2[v(q)+v(-q)]}}\right)^{m-\eta p} \cdot \sum_{\tau \in\{ \pm 1 ; 0\}} \frac{\mathrm{e}_{\tau}}{x^{n-\frac{b}{2}}} \cdot\left[H_{n ; x}^{\left(\left\{\epsilon_{t}\right\}\right)}\right]_{m, p, b, \tau} .
$$

The $\mathrm{O}\left(x^{-\infty}\right)$ appearing above holds in the $\left(L^{1} \cap L^{\infty}\right)\left(\mathscr{C}_{E}^{(\infty)}\right)$ sense. In order to lighten the formula, we have dropped the argument-dependent part. However, we do stress that the $\mathrm{O}\left(x^{-\infty}\right)$ as well as $\left[H_{n, x}^{\left(\left\{\epsilon_{t}\right\}\right)}\right]_{m, p, b, \tau}$ depend on the same set of variables as $H_{n ; x}^{\left(\epsilon_{t}\right)}$. Also, we agree upon $\boldsymbol{\eta}=1$ for $\lambda_{0}>q, \boldsymbol{\eta}=-1$ for $\left|\lambda_{0}\right|<q$ and we made use of the shorthand notation

$$
\mathrm{e}_{+}=\mathrm{e}^{i x u(q)} x^{-2 v(q)}, \mathrm{e}_{-}=\mathrm{e}^{i x u(-q)} x^{2 v(-q)} \quad \text { and } \quad \mathrm{e}_{0}=(1+\boldsymbol{\eta}) \mathrm{e}^{i x u\left(\lambda_{0}\right)} .
$$

Finally, the functions $\left[H_{n ; x}^{\left(\left\{\epsilon_{t}\right\}\right)}\right]_{m, p, b, \tau}$ are only supported on a small vicinity of the points $\pm q$ and $\lambda_{0}$. In such a case, the contour of integration reduces to an integration for each variable $z_{t}$ to a small circle $\partial \mathcal{D}_{0 ; v_{\tau}}$ around $v_{\tau}$ $\left(v_{ \pm}= \pm q, v_{0}=\lambda_{0}\right)$. Their dependence on $x$ is as follows. If a variable $z_{t}$ is integrated in a vicinity of $v_{\tau}$, The function $\left[H_{n, x}^{\left(\left\{\epsilon_{t}\right)\right.}\right]_{m, p, b, \tau}$ contains a fractional power of $x^{ \pm\left[2 v\left(z_{t}\right)-v\left(v_{\tau}\right)\right]}$, multiplied by a function of $z_{t}$ which has an asymptotic expansion into inverse powers of $x$. This asymptotic expansion holds on $\partial \mathcal{D}_{0 ; v_{\tau}}$. The coefficients in this asymptotic expansions contain poles at $z_{t}=v_{\tau}$. By computing the integrals associated to the terms in this asymptotic expansion through the poles at $z_{t}=v_{\tau}$ one obtains that function coefficients associated to $x^{-r}$ terms produce, in fine, a contribution that is a $(\ln x / x)^{r}$. Finally, the structure of these poles is such that, upon computing all the partial derivatives and for any holomorphic function $h$ in the vicinity of the points $\pm q, \lambda_{0}$, one should make the replacement:

$$
\sum_{\boldsymbol{t} \in J_{\{\vec{k}\}}} \epsilon_{t} h\left(z_{\boldsymbol{t}}\right) \hookrightarrow \boldsymbol{\eta} b\left(h\left(\lambda_{0}\right)-h(-q)\right)+(m-\boldsymbol{\eta} p)(h(q)-h(-q))+\left(\delta_{\tau ; 1}+\delta_{\tau ;-1}+(1+\boldsymbol{\eta}) \delta_{\tau ; 0} / 2\right) h\left(v_{\tau}\right) .
$$

There is one last property which we conjecture to be true for the detailed representation $(\overline{\mathrm{B} .51})$ of $H_{n ; x}^{\left(\left\{\epsilon_{t}\right\}\right)}$ but that has not been proven so far. Namely, 
Conjecture B.1 For a given $n$ the sum in (B.51) only contains those combinations of the integers $m, p, b$ and $\tau$ that satisfy to the constraint

$$
\left(m-\boldsymbol{\eta} p+\delta_{\tau, 1}\right)^{2}+b+\left(m+\boldsymbol{\eta}(b-p)-\delta_{\tau,-1}\right)^{2} \geq n .
$$

\section{Multidimensional Fredholm series for $\lim _{N \rightarrow+\infty} \rho_{N ; \mathrm{eff}}^{(m)}(x, t)$}

We begin this appendix by deriving the so-called discreet multidimensional Fredholm series representation for $\rho_{N ; \text { eff }}^{(m)}(x, t)$. We will prove in theorem $\mathrm{C}$.1 that this representation has a well defined thermodynamic limit that we denote $\rho_{\mathrm{eff}}^{(m)}(x, t)$. This analysis will allow us to provide in proposition C.1 yet another representation for the thermodynamic limit $\rho_{\mathrm{eff}}^{(m)}(x, t)$. This alternative representation for $\rho_{\mathrm{eff}}^{(m)}(x, t)$ is used in subsection 4.6 so as to construct the multidimensional Natte series for $\rho_{\mathrm{eff}}^{(m)}(x, t)$.

Theorem C.1 $\rho_{N ; \mathrm{eff}}^{(m)}(x, t)$ admits a well defined thermodynamic limit $\rho_{\mathrm{eff}}^{(m)}(x, t)$ that is given by a multidimensional Fredholm series

$$
\begin{aligned}
\rho_{\mathrm{eff}}^{(m)}(x, t) & =\sum_{n=0}^{m} c \frac{(-1)^{n}}{n !} \frac{\partial^{m}}{\partial \gamma^{m}} \int_{-q}^{q} \frac{\mathrm{d}^{n} \lambda}{(2 i \pi)^{n}} \oint_{\mathscr{C}_{q}} \frac{\mathrm{d}^{n} z}{(2 i \pi)^{n}} \int_{\mathscr{C}} \frac{\mathrm{d}^{n+1} y}{(2 i \pi)^{n+1}} \frac{\mathrm{e}^{i x \mathcal{U}\left(\left\{\lambda_{a}\right\}_{1}^{n} ;\left\{y_{a}\right\}_{1}^{n+1} \mid \gamma\right)} \prod_{k=1}^{n+1} f\left(y_{k}, \gamma v\left(y_{k}\right)\right)}{\prod_{k=1}^{n}\left(z_{k}-\lambda_{k}\right)\left(y_{k}-z_{k}\right)\left(y_{n+1}-\lambda_{k}\right)} \operatorname{det}_{n}\left[\frac{\left(y_{n+1}-z_{k}\right)}{z_{a}-\lambda_{b}}\right] \\
& \times\left.\frac{\prod_{a=1}^{n} \prod_{b=1}^{n+1}\left(y_{b}-\lambda_{a}-i c\right)\left(\lambda_{a}-y_{b}-i c\right)}{\prod_{a, b=1}^{n+1}\left(y_{a}-y_{b}-i c\right) \prod_{a, b=1}^{n}\left(\lambda_{a}-\lambda_{b}-i c\right)} \prod_{k=1}^{n}\left\{4 \sin ^{2}\left[\pi \gamma v\left(\lambda_{k}\right)\right]\right\} \frac{\operatorname{det}_{n}\left[\delta_{k \ell}+\gamma \widehat{V}_{k \ell}[v]\right] \operatorname{det}_{n}\left[\delta_{k \ell}+\gamma \overline{\bar{V}}_{k \ell}[v]\right]}{\operatorname{det}[I+\gamma R / 2 \pi] \operatorname{det}^{2}[I-K / 2 \pi]}\right|_{\gamma=0} .
\end{aligned}
$$

The function $f$ appearing above is supported on the contour $\mathscr{C}=\mathscr{C}_{E}^{(\infty)} \cup \widetilde{\mathscr{C}}_{q}$. The contour $\mathscr{C}_{q}$ is a small loop around $[-q ; q]$ whereas $\widetilde{\mathscr{C}}_{q}$ is a small loop around $\mathscr{C}_{q}$. Both $\mathscr{C}_{q}$ and $\widetilde{\mathscr{C}}_{q}$ lie below the curve $\mathscr{C}_{E}^{(\infty)}$ as depicted on Fig. 6. All of the aforementioned contours lie inside of the strip $U_{\delta}(2.15)$. The function $f$ is supported on $\mathscr{C}$ and reads

$$
f(y, v(y))=\mathbf{1}_{\mathscr{C}_{E}^{(\infty)}}(y)-\frac{1}{\mathrm{e}^{-2 i \pi v(y)}-1} \mathbf{1}_{\widetilde{\mathscr{C}}_{q}}(y) \quad \text { with } \quad \mathscr{C}=\mathscr{C}_{E}^{(\infty)} \cup \widetilde{\mathscr{C}}_{q}
$$

There $\mathbf{1}_{A}$ stands for the indicator function of the set A. The function $v$ appearing in the $n^{\text {th }}$-summand of (C.1) corresponds to the unique solution of the linear integral equation driven by the resolvent $R$ of the Lieb kernel (ie $[I-K / 2 \pi][I+R / 2 \pi]=I)$ :

$$
v(\lambda)+\gamma \int_{-q}^{q} \frac{\mathrm{d} \mu}{2 \pi} R(\lambda, \mu) v(\mu)=-Z(\lambda) / 2+\sum_{a=1}^{n} \phi\left(\lambda, \lambda_{a}\right)-\sum_{a=1}^{n+1} \phi\left(\lambda, y_{a}\right) .
$$

Hence, $v$ depends on the integration variables $\lambda_{a}$ (with $a=1, \ldots, n$ ) and $y_{a}$ (with $a=1, \ldots, n+1$ ), ie $v(\lambda) \equiv$ $v\left(\lambda \mid\left\{\lambda_{a}\right\}_{1}^{n} ;\left\{y_{a}\right\}_{1}^{n+1}\right)$. We kept this dependence implicit in (C.1) so as to shorten the formulae. The entries of the finite-size determinants are as defined in (4.8). They depend on the same set of auxiliary variables as $v$. Finally, we agree upon

$$
\mathcal{U}\left(\left\{\lambda_{a}\right\}_{1}^{n} ;\left\{y_{a}\right\}_{1}^{n+1} \mid \gamma\right)=\sum_{a=1}^{n+1} u_{0}\left(y_{a}\right)-\sum_{a=1}^{n} u_{0}\left(\lambda_{a}\right)+(1-\gamma) \int_{-q}^{q} u_{0}^{\prime}(\lambda) v(\lambda) \mathrm{d} \lambda
$$




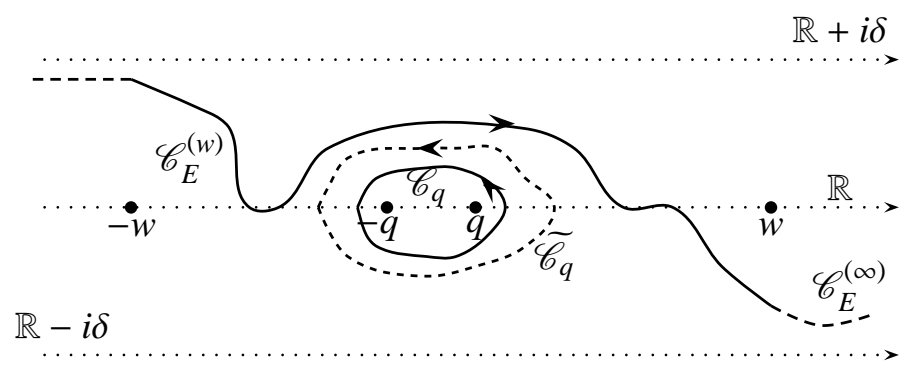

Figure 6: The contour $\mathscr{C}_{E}^{(w)}$ consists of the solid line. The contour $\mathscr{C}_{E}^{(\infty)}$ corresponds to the union of the solid and dotted lines. The loop $\mathscr{C}_{q}$ is depicted in solid lines whereas the loop $\widetilde{\mathscr{C}}_{q}$ is depicted in dotted lines.

Proof-

In order to implement the substitution of the operators $\partial_{S_{p}}$ and $\partial_{\eta_{j, p}}(c f$ section 4.3) in the representation (4.41) we introduce, exactly as it was done in the proof of theorem 4.1, the functions $\widetilde{E}_{-}(\lambda)$ (4.51) (whose definition involves the functions $\widetilde{g}=\widetilde{g}_{1, s}+\widetilde{g}_{2, r} c f(4.52)$ ) as well as $v_{s}(4.36)$ and $\varpi_{r}(4.32)$.

We then consider the discreet Fredholm series representation for $\partial_{\gamma}^{m} X_{N}\left[\gamma v_{s}, \widetilde{E}_{-}^{2}\right]_{\gamma=0}$ obtained in proposition B.4. This will allow us to compute the relevant Taylor coefficients ( $c f$ subsection 4.3 equation (4.20) and (4.21) arising in the representation 4.41 for $\rho_{N ; \text { eff }}^{(m)}(x, t)$. One has that

$$
\begin{aligned}
\frac{\partial^{m}}{\partial \gamma^{m}}\left\{\prod_{a=1}^{N+1} \widetilde{E}_{-}^{2}\left(\mu_{a}\right) \cdot \prod_{a=1}^{N} \widetilde{E}_{-}^{-2}\left(\lambda_{a}\right) \cdot X_{N}\left[\gamma v_{s}, \widetilde{E}_{-}^{2}\right] \widehat{\mathscr{G}}_{\gamma ; 2 A_{L}}^{(\beta)}\left[\varpi_{r}\right]\right\}_{\mid \gamma=0}= \\
\sum_{n=0}^{m} i \frac{(-1)^{n}}{n !} \sum_{\substack{i_{1}, \ldots, i_{n} \\
i_{a} \in \llbracket 1 ; N \rrbracket}} \oint_{\mathscr{C}_{q}} \frac{\mathrm{d}^{n} z}{(2 i \pi)^{2 n}} \int_{\mathscr{C}(L)} \frac{\mathrm{d}^{n+1} y}{(2 i \pi)^{n+1}} \widetilde{\mathcal{L}}_{\Gamma^{(L)}}^{(m)}\left[\mathcal{F}_{i_{1}, \ldots i_{n}} \widehat{\mathscr{G}}_{\gamma ; 2 A_{L}}^{(\beta)}\right] .
\end{aligned}
$$

The contours $\mathscr{C}^{(L)}, \mathscr{C}_{q}$ have been defined in proposition B.4. We stress that the summation over $n$ in (C.5) could have been stopped at $n=m$ since, prior to taking the $\gamma$-derivative at $\gamma=0$, the $n^{\text {th }}$ term of the series (B.41) is a smooth function of $\gamma$ that behaves as $\mathrm{O}\left(\gamma^{n}\right)$. We have set

$$
\mathcal{F}_{i_{1}, \ldots, i_{n}}\left[\gamma v_{s}\right]=\prod_{k=1}^{n}\left\{\frac{4 \sin ^{2}\left[\pi \gamma v_{s}\left(\lambda_{i_{k}}\right)\right]}{L \xi_{\gamma \gamma_{s}}^{\prime}\left(\lambda_{i_{k}}\right)\left(z_{k}-\lambda_{i_{k}}\right)}\right\} \frac{\prod_{k=1}^{n+1} f^{(L)}\left(y_{k}, \gamma v_{s}\left(y_{k}\right)\right)}{\prod_{k=1}^{n}\left(y_{n+1}-\lambda_{i_{k}}\right)\left(y_{k}-z_{k}\right)} \times \operatorname{det}_{n}\left[\frac{y_{n+1}-z_{j}}{z_{j}-\lambda_{i_{k}}}\right] \mathrm{e}^{i x \mathcal{U}^{(L)}\left(\left\{\lambda_{k}\right\} ;\left\{\mu_{k}\right\} ;\left\{y_{k}\right\} \mid \gamma\right)}
$$

the function $f^{(L)}(y, v(y))$ is given in $(\overline{\mathrm{B} .42})$ and we have set

$$
\mathcal{U}^{(L)}\left(\left\{\lambda_{k}\right\} ;\left\{\mu_{k}\right\} ;\left\{y_{k}\right\} \mid \gamma\right)=\sum_{k=1}^{n+1} u\left(y_{k}\right)-\sum_{k=1}^{n} u\left(\mu_{i_{k}}\right)-u\left(\mu_{N+1}\right)+\sum_{\substack{k=1 \\ \neq i_{1}, \ldots, i_{n}}}^{N} u\left(\lambda_{k}\right)-u\left(\mu_{k}\right)
$$


Last but not least,

$$
\begin{aligned}
\widetilde{\mathcal{L}}_{\Gamma^{(L)}}^{(m)}\left[\mathcal{F}_{i_{1}, \ldots i_{n}} \mathscr{G}_{\gamma ; 2 A_{L}}^{(\beta)}\right]= & \sum_{n_{1}, \ldots, n_{s}}^{m} \prod_{p=1}^{s}\left\{\frac{a_{p}^{n_{p}}}{n_{p} !}\right\} \frac{\partial^{m}}{\partial \gamma^{m}}\left\{\frac{\prod_{k=1}^{n+1} \mathrm{e}^{\widetilde{g}_{1, s}\left(y_{k}\right)}}{\mathrm{e}^{\widetilde{g}_{1, s}\left(\mu_{N+1}\right)} \prod_{k=1}^{n} \mathrm{e}^{\widetilde{g}_{1, s}\left(\mu_{i_{k}}\right)}} \prod_{p=1}^{s}\left\{\Gamma^{(L)}\left[\gamma v_{s}\right]\left(t_{p}\right)\right\}^{n_{p}}\right. \\
& \left.\left.\times \prod_{j=1}^{N+1} \mathrm{e}^{-\widetilde{g}_{2, r}\left(\mu_{j}\right)} \prod_{\substack{j=1 \\
\neq i_{1}, \ldots, i_{n}}}^{N} \mathrm{e}^{\widetilde{g}_{2, r}\left(\lambda_{j}\right)} \prod_{j=1}^{n+1} \mathrm{e}^{\widetilde{g}_{2, r}\left(y_{j}\right)} \cdot \mathcal{F}_{i_{1}, \ldots, i_{n}}\left[\gamma \widetilde{\gamma}_{s}\right]\right]_{\substack{\gamma=0 \\
s_{a}=0}} \widetilde{\mathscr{G}}_{\gamma ; 2 A_{L}}^{(\beta)}\left[\varpi_{r}\right]\right\}_{\mid \gamma=0} .
\end{aligned}
$$

The functional $\Gamma^{(L)}$ is evaluated at the discretization points ( $c f$ definition 4.2, subsection 4.4 and subsection 4.2) $t_{p}, p=1, \ldots, s$ for the contour $\mathscr{C}_{\text {out }}$ encircling the compact $K_{2 q} . \mathscr{C}_{\text {out }}$ has been depicted in the lhs of Fig. 1 The functional $\Gamma^{(L)}$ reads

$$
\Gamma^{(L)}[v](\mu)=\sum_{\substack{j=1 \\ j \neq i_{1}, \ldots, i_{n}}}^{N} \phi\left(\mu, \mu_{j}\right)-\phi\left(\mu, \lambda_{j}\right) \quad \text { with } \quad \mu_{j} \text { and } \lambda_{j} \text { defined by } \xi\left(\mu_{j}\right)=j / L \text { and } \xi_{v}\left(\lambda_{j}\right)=j / L \text {. (C.9) }
$$

We do stress that the variables $y_{k}$, with $k=1, \ldots, n+1$, and $\mu_{p}$ or $\lambda_{p}$ with $p=1, \ldots, N+1$ appearing in (C.5)-(C.8) are all located inside of the compact $K_{2 A_{L}}$, where $A_{L}$ is such that $L \xi\left(-A_{L}\right)=-w_{L}-1 / 2$. As a consequence, the singularities at $\lambda=t_{i, p}$ of the functions $\widetilde{g}_{2, r}(\lambda)(\bar{C} .8)$ are always disjoint from the variables $y_{k}, \mu_{p}$ or $\lambda_{i_{b}}$. Indeed, $t_{1, p}$ and $t_{2, p}$ with $p=1, \ldots, r$ stand for discretization points of the contour $\mathscr{C}_{\text {out }}$ in appearing in the $r h s$ of Fig. 1, $c f$ subsection 4.4 These two contours are such that $\mathrm{d}\left(\mathscr{C}_{\text {out }} /\right.$ in,$\left.K_{2 A_{L}}\right)>0$ uniformly in $L$.

According to the prescription that has been adopted in section 4.3, one has to compute the $m^{\text {th }} \gamma$-derivative of representation (C.8) prior to implementing the operator substitution. For this, consider any smooth function $w(\gamma)$ such that $w(\gamma)=\mathrm{O}\left(\gamma^{n}\right)$ at $\gamma=0$. By applying the Faa-dí-Bruno formula, we get that

$$
\begin{aligned}
& \frac{1}{m !} \frac{\partial^{m}}{\partial \gamma^{m}}\left\{w(\gamma) \prod_{\substack{a=1 \\
\neq i_{1}, \ldots, i_{n}}}^{N} \mathrm{e}^{\widetilde{g}_{2, r}\left(\lambda_{a}\right)} \widehat{\mathscr{G}}_{\gamma ; 2 A_{L}}^{(\beta)}\left[\varpi_{r}\right]\right\}_{\mid \gamma=0}=\sum_{\left\{\ell_{a}\right\}} \frac{w^{\left(\ell_{N+1}\right)}(0)}{\ell_{0} ! \ell_{N+1} !} \prod_{\substack{p=1 \\
\neq i_{1}, \ldots, i_{n}}}^{N} \frac{1}{\ell_{p} !} \frac{\partial^{\ell_{p}}}{\partial \gamma^{\ell_{p}}}\left\{\mathrm{e}^{\widetilde{g}_{2, r}\left(\lambda_{p}\right)}\right\}_{\mid \gamma=0} \times \frac{\partial^{\ell_{0}}}{\partial \gamma^{\ell_{0}}}\left\{\widehat{\mathscr{G}}_{\gamma ; 2 A_{L}}^{(\beta)}\left[\varpi_{r}\right]\right\}_{\mid \gamma=0} \\
& =\sum_{\left\{\ell_{a}\right\}}, \frac{w^{\left(\ell_{N+1}\right)}(0)}{\ell_{0} ! \ell_{N+1} !} \sum_{\left\{k_{a, j}\right\}}^{\prime} \prod_{\substack{p=1 \\
\neq i_{1}, \ldots, i_{n}}}^{N}\left\{\left.\frac{\partial^{\left|\boldsymbol{k}_{p}\right|} \mathrm{e}^{\widetilde{g}_{2, r}\left(\tau_{p}\right)}}{\partial \tau_{p}^{\left|\boldsymbol{k}_{p}\right|}}\right|_{\tau_{p}=\mu_{p}} \times \prod_{j=1}^{\ell_{p}}\left(\frac{\lambda_{p}^{(j)}}{j !}\right)^{k_{p, j}}\right\} \times \frac{\partial^{\ell_{0}}}{\partial \gamma^{\ell_{0}}}\left\{\widehat{\mathscr{G}}_{\gamma ; 2 A_{L}}^{(\beta)}\left[\varpi_{r}\right]\right\}_{\mid \gamma=0} .
\end{aligned}
$$

There the, in front of the sums indicates that these are constrained. The first sums runs through all choices of $N+2$ integers $\ell_{p} \geq 0$ such that

$$
\ell_{i_{p}}=0, \quad \text { for } \quad p=1, \ldots, n, \quad \ell_{N+1} \geq n \quad \text { and } \quad \sum_{p=0}^{N+1} \ell_{p}=m
$$

The second sum runs through all the possible choices of sequences of integers $k_{p, j}$ with

$$
p=0, \ldots, N \text { and } j=1, \ldots, \ell_{p} \quad \text { such that } \sum_{j=1}^{\ell_{p}} j k_{p, j}=\ell_{p} .
$$

Finally, we agree upon

$$
\left|\boldsymbol{k}_{p}\right|=\sum_{j=1}^{\ell_{p}} k_{p, j} \quad \text { and have set } \quad \lambda_{p}^{(j)}=\partial_{\gamma_{p}}^{j}\left[\lambda_{p}\left(\gamma_{p}\right)\right]_{\mid \gamma_{p}=0} \quad \text { with } \quad \xi_{\gamma_{p} \nu_{s}}\left(\lambda_{p}\right)=p / L
$$


In (C.13), we have explicitly insisted on the fact that $\lambda_{p}$ is a function of the parameter $\gamma_{p}$. By substituting the representation (C.10) on the level of (C.5)-(C.8), one can implement the operator substitution $a_{k} \hookrightarrow \partial_{S_{k}}$ and $b_{j, k} \hookrightarrow \partial_{\eta_{j, k}}$ on the level of (C.8).

The functionals $\mathcal{F}_{i_{1}, \ldots, i_{n}}$ and $\widehat{\mathscr{G}}_{\gamma ; 2 A_{L}}^{(\beta)}$ are regular in the sense of definition 4.1. Moreover, as $L$ and hence $2 A_{L}$ are large enough, and $\beta_{0}$ defining $\widetilde{\boldsymbol{U}}_{\beta_{0}}$ is chosen in such a specific way th the constant of regularity $C_{\mathscr{G}_{2 A_{L}}}$ of $\widehat{\mathscr{G}}_{\gamma ; 2 A_{L}}^{(\beta)}$ satisfies (4.30), one gets that

$$
\left\{\varsigma_{a}\right\}_{1}^{s} \mapsto \mathcal{F}_{i_{1}, \ldots, i_{n}}\left[\gamma v_{s}\left(* \mid\left\{\varsigma_{a}\right\}\right)\right] \quad \text { and } \quad\left\{\eta_{1, p}\right\}_{1}^{r} \cup\left\{\eta_{2, p}\right\}_{1}^{r} \mapsto \widehat{\mathscr{G}}_{\gamma ; 2 A_{L}}^{(\beta)}\left[\varpi_{r}\left(* \mid\left\{\eta_{a, p}\right\}\right)\right]
$$

are holomorphic in respect to $\left\{\varsigma_{a}\right\}_{1}^{s} \in \mathcal{N}_{0}^{s},\left\{\eta_{1, p}\right\}_{1}^{r} \cup\left\{\eta_{2, p}\right\}_{1}^{r} \in \mathcal{N}_{0}^{2 r}$, where $\mathcal{N}_{0}$ is an $r$ and $s$ independent neighborhood of $0 \in \mathbb{C}$. As the constant of regularity $C_{\mathscr{G}_{2 A}}$ is large enough and $|\gamma|$ can be taken small enough, the size of the neighborhood $\mathcal{N}_{0}$ is large enough in order to ensure the convergence of the series of differential operators issuing from the exponentials $\mathrm{e}^{\widetilde{g}_{1, s}}$ and $\mathrm{e}^{\widetilde{g}_{2, r}}$, once upon the operator substitution is carried out. In virtue of corollary D.1, and similarly to the summations (4.55)-(4.56), the action of the translation operators can be computed directly under the integral sign in (C.5) (the integration contours being Cartesian products of one dimensional compact curves) and prior to taking the partial $\tau_{p}$ or $\gamma$-derivatives in (C.10). There are also the differential operators arising from the substitutions $a_{p} \hookrightarrow \partial_{S_{p}}$ in (C.8) for those parameters $a_{p}$ that are written down explicitly. The resulting $\partial_{S_{p}}$-derivatives should appear outside of the integrals that are written down in (C.5). However, the integrand of these compactly supported integrals is a continuous function of the integration variables that is holomorphic in respect to $\left\{\varsigma_{p}\right\}_{1}^{s}$, this uniformly in respect to the integration variables. As a consequence, one can exchange the derivation and integration symbols in this case as well.

Note that the constraints (C.13) on the $k_{p, j}$ 's ensure that in (C.10) there is at most $m-n$ integers $k_{p, j}$ that differ from zero. As a consequence, there will be at most $m$ translation operators in respect to the $\eta_{i, k}$ variables to take into account once that the operator substitution is made. More precisely, the substitution $b_{j, k} \hookrightarrow \partial_{\eta_{j, k}}$ shifts the parameters $\eta_{j, k}$ in $\varpi_{r}\left(\lambda,\left\{\eta_{j, k}\right\}\right)$ (4.32) to the below value

$$
\eta_{j, k}=\sum_{p=1}^{n+1} \frac{1}{t_{j, k}-y_{p}}-\sum_{p=1}^{n} \frac{1}{t_{j, k}-\mu_{i_{p}}}-\frac{1}{t_{j, k}-\mu_{N+1}}+\sum_{\substack{p=1 \\ \ell_{p} \neq 0}}^{N} \frac{1}{t_{j, k}-\tau_{p}}-\frac{1}{t_{j, k}-\mu_{p}}
$$

where the ulimate sum in (C.15) only involves $m$ terms at most. Under the substitution $a_{p} \hookrightarrow \partial_{\varsigma_{a}}$, the exponentials in (C.8) produce a translation of the function $v_{s} \hookrightarrow \widetilde{v}_{s}$, where

$$
\widetilde{v}_{s}\left(\lambda ;\left\{\zeta_{a}\right\}\right)=v_{s}\left(\lambda ;\left\{\varsigma_{a}\right\}\right)+\sum_{j=1}^{s} \frac{t_{j+1}-t_{j}}{2 i \pi\left(t_{j}-\lambda\right)}\left\{\phi\left(t_{j}, \mu_{N+1}\right)-\phi\left(t_{j}, y_{n+1}\right)+\sum_{a=1}^{n} \phi\left(t_{j}, \mu_{i_{a}}\right)-\phi\left(t_{j}, y_{a}\right)\right\} .
$$

After carrying out all these manipulations, we are led to the representation

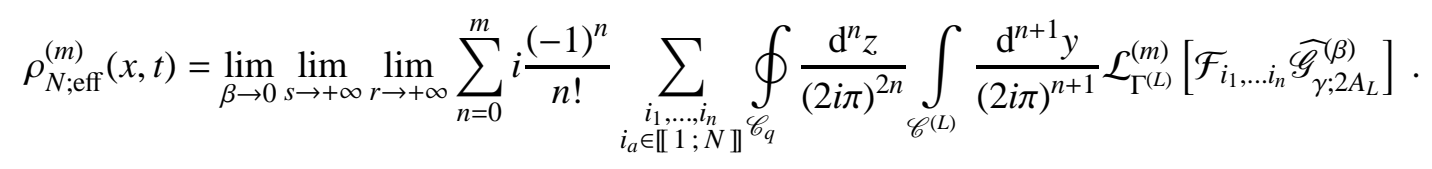

\footnotetext{
${ }^{\dagger}$ in particular it depends on $\mathrm{L}, c f$ lemma A.2 However, $|\gamma \beta| \cdot L^{-1}$ is still very small $c f$ lemma A.2
} 
where $\mathcal{L}_{\Gamma^{(L)}}^{(m)}$ is a truncated Lagrange series:

$$
\begin{aligned}
\mathcal{L}_{\Gamma^{(L)}}^{(m)}\left[\mathcal{F}_{i_{1}, \ldots i_{n}} \mathscr{G}_{\gamma ; 2 A_{L}}^{(\beta)}\right] & =\sum_{n_{1}, \ldots, n_{s}}^{m} \prod_{p=1}^{s}\left\{\frac{1}{n_{p} !} \frac{\partial^{n_{p}}}{\partial \varsigma_{p}^{n_{p}}}\right\} \sum_{\left\{\ell_{a}\right\}}^{\prime} \frac{m !}{\ell_{0} ! \ell_{N+1} !} \frac{\partial^{\ell_{N+1}}}{\partial \gamma^{\ell_{N+1}}}\left\{\prod_{p=1}^{s}\left\{\Gamma^{(L)}\left[\gamma \widetilde{v}_{s}\right]\left(t_{p}\right)\right\}^{n_{p}} \mathcal{F}_{i_{1}, \ldots, i_{n}}\left[\gamma \widetilde{\gamma}_{s}\right]\right\}_{\mid \gamma=0} \\
& \times \sum_{\left\{k_{a, j}\right\}}^{\prime} \prod_{\substack{a=1 \\
\neq i_{1}, \ldots, i_{n}}}^{N} \prod_{j=1}^{\ell_{a}}\left(\frac{\lambda_{a}^{(j)}}{j !}\right)^{k_{a, j}} \frac{\partial^{\ell_{0}}}{\partial \gamma^{\ell_{0}}} \prod_{\substack{a=1 \\
\neq i_{1}, \ldots, i_{n}}}^{N} \frac{\partial^{\left|\boldsymbol{k}_{a}\right|}}{\partial \tau_{a}^{\left|\boldsymbol{k}_{a}\right|}} \times\left\{\widehat{\mathscr{G}}_{\gamma ; 2 A_{L}}^{(\beta)}\left[\varpi_{r}\left(* \mid\left\{\eta_{i, k}\right\}\right)\right]\right\}_{\substack{\gamma=0 \\
\tau_{a}=\mu_{a}}} . \quad \text { (C.18) }
\end{aligned}
$$

We now take the $r \rightarrow+\infty$ limit of $(\overline{C .17})-(\overline{C .18})$. The very construction of $\varpi_{r}\left(\lambda \mid\left\{\eta_{j, k}\right\}\right)$ along with the choice of parameters $\eta_{i, k}$ given by $(\mathrm{C} .15)$ associated with the fact that $\mathscr{G}_{\gamma ; 2 A_{L}}^{(\beta)}$ is a regular functional with a sufficiently large regularity constant, leads to ( $c f$ proof of proposition D.1)

$$
\lim _{r \rightarrow+\infty} \widehat{\mathscr{G}}_{\gamma ; 2 A_{L}}^{(\beta)}\left[\varpi_{r}\right]=\widehat{\mathscr{G}}_{\gamma ; 2 A_{L}}^{(\beta)}\left[H\left(* \mid \begin{array}{c}
\left\{y_{a}\right\}_{1}^{n+1} \cup\left\{\tau_{a}\right\}_{a: \ell_{a} \neq 0} \\
\left\{\mu_{i_{a}}\right\}_{1}^{n} \cup\left\{\mu_{N+1}\right\} \cup\left\{\mu_{a}\right\}_{a: \ell_{a} \neq 0}
\end{array}\right)\right]
$$

this uniformly in $y_{a}, \mu_{a} \lambda_{a}$ and $\tau_{a}$ belonging to $K_{2 A_{L}}$. This uniform convergence also holds in respect to any finite order partial derivative in these parameters. The uniformness of this limit in respect to the integration parameters occurring in (C.17) allows one to take it directly under the integral sign over a compact domain.

As a consequence, we get that

$$
\begin{aligned}
\left.\lim _{r \rightarrow+\infty} \sum_{\left\{k_{a, j}\right\}}\right\} \prod_{\substack{a=1 \\
\neq i_{1}, \ldots, i_{n}}}^{N} \prod_{j=1}^{\ell_{a}}\left(\frac{\lambda_{a}^{(j)}}{j !}\right)^{k_{a, j}} \frac{\partial^{\ell_{0}}}{\partial \gamma^{\ell_{0}}} \prod_{\substack{a \\
\neq i_{1}, \ldots, i_{n}}}^{N} \frac{\partial^{\left|\boldsymbol{k}_{a}\right|}}{\partial \tau_{a}^{\left|\boldsymbol{k}_{a}\right|}} \times\left\{\widehat{\mathscr{G}}_{\gamma ; 2 A_{L}}^{(\beta)}\left[\varpi_{r}\left(* \mid\left\{\eta_{j, k}\right\}\right)\right]\right\}_{\left.\right|_{\tau_{a}=\mu_{a}} ^{\gamma=0}} \\
=\prod_{\substack{a=0 \\
\neq i_{1}, \ldots, i_{n}}}^{N} \frac{1}{\ell_{a} !} \frac{\partial^{\ell_{a}}}{\partial \gamma_{a}^{\ell_{a}}}\left\{\begin{array}{c}
\widehat{\mathscr{G}}_{\gamma_{0} ; 2 A_{L}}^{(\beta)} \\
\gamma^{\prime}
\end{array}\left[H\left(* \mid \begin{array}{c}
\left\{y_{a}\right\}_{1}^{n+1} \cup\left\{\lambda_{a}\left(\gamma_{a}\right)\right\}_{1}^{N} \\
\left\{\mu_{a}\right\}_{1}^{N+1} \cup\left\{\lambda_{i_{a}}\left(\gamma_{i_{a}}\right)\right\}_{1}^{n}
\end{array}\right)\right]\right\}_{\mid \gamma_{a}=0} .
\end{aligned}
$$

To get the rhs of this equality we have, in addition to exchanging the limits and derivatives, applied the Faa-díBruno formula backwards. The constant of regularity of $\widehat{\mathscr{G}}_{\gamma_{0} ; 2 A_{L}}^{(\beta)}$ being large enough, the action of $\widehat{\mathscr{G}}_{\gamma_{0} ; 2 A_{L}}^{(\beta)}$ on $H$ as written in the second line of $(\overline{C .20})$ is indeed well defined.

After collecting the various $\gamma_{a}$ derivatives into a single one, we arrive to the representation

$$
\lim _{r \rightarrow+\infty} \mathcal{L}_{\Gamma^{(L)}}^{(m)}\left[\mathcal{F}_{i_{1}, \ldots i_{n}} \widehat{\mathscr{G}}_{\gamma ; 2 A_{L}}^{(\beta)}\right]=\sum_{n_{1}, \ldots, n_{s}}^{m} \prod_{a=1}^{s}\left\{\frac{1}{n_{a} !} \frac{\partial^{n_{a}}}{\partial \varsigma_{a}^{n_{a}}}\right\} \frac{\partial^{m}}{\partial \gamma^{m}}\left\{\prod_{a=1}^{s}\left\{\Gamma^{(L)}\left[\gamma \widetilde{\gamma}_{s}\right]\left(t_{a}\right)\right\}^{n_{a}} \cdot \mathcal{J}_{i_{1}, \ldots, i_{n}}\left[\gamma \widetilde{\gamma}_{s}\right]\right\}_{\substack{\gamma=0 \\ S_{a}=0}}
$$

where we have set

$$
\mathcal{J}_{i_{1}, \ldots, i_{n}}\left[\widetilde{\gamma \widetilde{v}_{s}}\right]=\mathcal{F}_{i_{1}, \ldots, i_{n}}\left[\gamma \widetilde{\gamma}_{s}\right] \widehat{\mathscr{G}}_{\gamma ; 2 A_{L}}^{(\beta)}\left[H\left(* \mid \begin{array}{c}
\left\{y_{a}\right\}_{1}^{n+1} \cup\left\{\lambda_{a}\right\}_{1}^{N} \\
\left\{\mu_{a}\right\}_{1}^{N+1} \cup\left\{\lambda_{i_{a}}\right\}_{1}^{n}
\end{array}\right)\right]
$$

Since, no confusion is possible on the level of (C.21)-(C.22), the $\gamma$-dependence of the parameters $\lambda_{p}, p=1, \ldots, N$ is kept implicit again.

The truncated $s$-dimensional Lagrange series (C.21) together with its $s \rightarrow+\infty$ limit has been studied in appendix D.5.2. It follows from the latter analysis that the $s \rightarrow+\infty$ limit is uniform in respect to the parameters $\left(\left\{y_{k}\right\}_{1}^{n+1},\left\{z_{k}\right\}_{1}^{n}\right)$ on which $\mathcal{J}_{i_{1}, \ldots, i_{n}}$ depends. Therefore, this limit can be taken under the integrals signs. Similarly, 
one can exchange the limit with the $m^{\text {th }} \gamma$-derivative symbol. It follows from the results gathered in appendix D.5.2 that

$$
\lim _{s \rightarrow+\infty} \lim _{r \rightarrow+\infty} \mathcal{L}_{\Gamma^{(L)}}^{(m)}\left[\mathcal{F}_{i_{1}, \ldots i_{n}} \mathscr{G}_{\gamma ; 2 A_{L}}^{(\beta)}\right]=\frac{\partial^{m}}{\partial \gamma^{m}}\left\{\mathcal{J}_{i_{1}, \ldots, i_{n}}\left[\gamma \nu^{(L)}\right] \cdot \operatorname{det}_{\mathscr{C}_{q}}^{-1}\left[I-\gamma \frac{\delta \Gamma^{(L)}[\rho]}{\delta \rho(\zeta)}(\mu)\right]_{\rho=\gamma \gamma^{(L)}}\right\}_{\mid \gamma=0} .
$$

The answer is expressed with the help of $v^{(L)}$, the unique solution (for $\gamma$-small enough) to the non-linear integral equation driven by the functional $\Gamma^{(L)}$ :

$$
v^{(L)}(\lambda)=(i \beta-1 / 2) Z(\lambda)-\phi(\lambda, q)+\sum_{a=1}^{N+1} \phi\left(\lambda, \mu_{a}\right)-\sum_{a=1}^{n+1} \phi\left(\lambda, y_{a}\right)-\sum_{\substack{a=1 \\
\neq i_{1}, \ldots, i_{n}}}^{N} \phi\left(\lambda, \lambda_{a}\right) \quad \text { with } \quad\left\{\begin{array}{c}
\lambda_{a}=\xi_{\gamma v^{(L)}}^{-1}(a / L) \\
\mu_{a}=\xi^{-1}(a / L)
\end{array} .\right.
$$

Also, in (C.23), appears the Fredholm determinant of the linear integral operator acting on a small loop $\mathscr{C}_{q}$ around $[-q ; q]$ whose kernel is given in terms of the functional derivative $\delta \Gamma^{(L)}[\rho](\mu) / \delta \rho(\zeta)$. The definition of the functional derivative is given in (D.11).

Lemma A.4 allows one to reexpress the functional $\widehat{\mathscr{G}}_{\gamma ; 2 A_{L}}^{(\beta)}$ appearing $(\overline{\mathbf{C} .22})$ in the case where the parameters $\lambda_{a}$ and $\mu_{a}$ are defined exactly as in (C.24) in terms of the unique solution $v^{(L)}$.

This leads to the below representation:

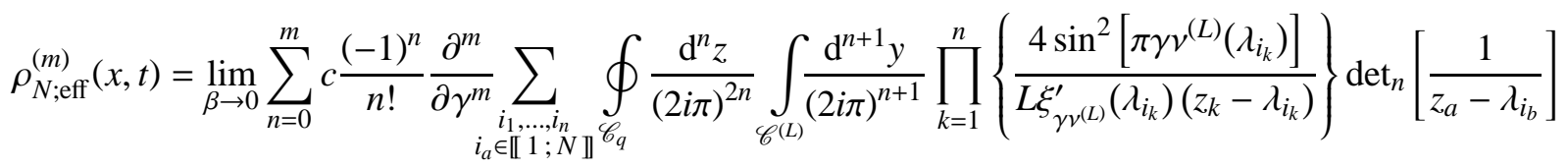

$$
\begin{aligned}
& \times \prod_{k=1}^{n}\left(\frac{y_{n+1}-z_{k}}{\left(y_{k}-z_{k}\right)\left(y_{n+1}-\lambda_{i_{k}}\right)}\right) \frac{\mathrm{e}^{i x \mathcal{U}^{(L)}\left(\left\{\lambda_{a}\right\}_{1}^{N} ;\left\{\mu_{a}\right\}_{1}^{N+1} ;\left\{y_{a}\right\}_{1}^{n+1} \mid \gamma\right)}}{\operatorname{det}_{\mathscr{C}_{q}}\left[I-\gamma \frac{\delta \Gamma^{(L)}[\rho]}{\delta \rho(\zeta)}(\mu)\right]_{\rho=\gamma v^{(L)}}} \frac{\prod_{a=1}^{n+1} \prod_{b=1}^{n+1}\left(y_{a}-y_{b}-i c\right) \prod_{a, b=1}^{n}\left(\lambda_{i_{a}}-\lambda_{i_{b}}-i c\right)}{\left.\lambda_{i_{a}}-i c\right)\left(\lambda_{i_{a}}-y_{b}-i c\right)} \\
& \times \frac{\prod_{k=1}^{n+1} f^{(L)}\left(y_{k}, \gamma v^{(L)}\left(y_{k}\right)\right)}{\operatorname{det}_{N+1}\left[\Xi^{(\mu)}[\xi]\right] \operatorname{det}_{N}\left[\Xi^{(\lambda)}\left[\xi_{\gamma v^{(L)}}\right]\right]} \cdot\left(\operatorname{det}_{n}\left[\delta_{k \ell}+\gamma \widehat{V}_{k \ell}\left[\gamma v^{(L)}\right]\right] \operatorname{det}_{n}\left[\delta_{k \ell}+\gamma \widehat{\bar{V}}_{k \ell}\left[\gamma v^{(L)}\right]\right]\right)\left(\left\{\lambda_{i_{a}}\right\}_{1}^{n},\left\{y_{a}\right\}_{1}^{n+1}\right)
\end{aligned}
$$

Above we have written down the dependence of both determinants on $\left\{\lambda_{i_{a}}\right\}$ and $\left\{y_{a}\right\}$ as a common argument.

There is no problem to carry out the analytic continuation in (C.30) from $\beta \in \widetilde{\boldsymbol{U}}_{\beta_{0}}$ up to $\beta=0$ : the potential singularities that could appear in the determinants are canceled by the prefactor $\prod_{k=1}^{n} \sin ^{2}\left[\pi \gamma v^{(L)}\left(\lambda_{i_{k}}\right)\right]$. From now on, we can thus set $\beta=0$

In order to prove the theorem, it remains to take the thermodynamic limit of (‥30) at $\beta=0$.

\section{$L \rightarrow+\infty$ behavior of $v^{(L)}$}

It was shown in this appendix that $v^{(L)}$ admits a large $L$ asymptotic expansion $v^{(L)}(\lambda)=v\left(\lambda \mid\left\{\lambda_{i_{a}}\right\}_{1}^{n} ;\left\{y_{a}\right\}_{1}^{n+1}\right)+$ $\mathrm{O}\left(L^{-1}\right)$. There the $\mathrm{O}$ is holomorphic and uniform in some open neighborhood of the real axis and the function $v(\lambda)=v\left(\lambda \mid\left\{\lambda_{i_{a}}\right\}_{1}^{n},\left\{y_{a}\right\}_{1}^{n+1}\right)$ stands for the unique solution to the linear integral equation (C.3) (here we have already set $\beta=0$ ).

As all of the functions we deal with are smooth functions of $v^{(L)}$, we are thus able to replace everywhere $v^{(L)}$ by $v$, up to $\mathrm{O}\left(L^{-1}\right)$ corrections. 
Building on the large $L$ asymptotics of $\Gamma^{(L)}$ and $v^{(L)}$ it is shown in subsection D.5.2, (D.43)-(D.44), that

$$
\operatorname{det} \mathscr{C}_{q}\left[I-\gamma \frac{\delta \Gamma^{(L)}[\rho]}{\delta \rho(\zeta)}(\mu)\right]_{\rho=\gamma v^{(L)}}=\operatorname{det}_{[-q ; q]}\left[I+\gamma \frac{R}{2 \pi}\right]\left(1+\mathrm{O}\left(L^{-1}\right)\right),
$$

with a $\mathrm{O}$ that has the same uniformness properties as stated before. Above, we did not insist that the Fredholm determinant $\operatorname{det}[I+\gamma R / 2 \pi]$ corresponds to an action on $[-q ; q]$.

\section{$L \rightarrow+\infty$ limit of $\mathcal{U}^{(L)}$}

The thermodynamic limit of $\mathcal{U}^{(L)}\left(\left\{\lambda_{a}\right\} ;\left\{\mu_{a}\right\} ;\left\{y_{a}\right\} \mid \gamma\right)$ is readily computed by using that

$$
\xi\left(\mu_{a}\right)-\xi_{\gamma \nu^{(L)}}\left(\lambda_{a}\right)=0=-\frac{\gamma \nu^{(L)}\left(\mu_{a}\right)}{L}+\frac{p^{\prime}\left(\mu_{a}\right)}{2 \pi}\left(\mu_{a}-\lambda_{a}\right)+\mathrm{O}\left(L^{-2}\right) .
$$

The remainder $\mathrm{O}\left(L^{-2}\right)$ is uniform in $a \in \llbracket 1 ; N \rrbracket$ and holomorphic in respect to the variables $y_{a}$ and $z_{a}$ belonging to $U_{\delta / 2}$. By using the Euler-MacLaurin formula, the linear integral equation (C.3) satisfied by $v$ and the integral representation (2.13) for $u$ one gets that

$$
\mathcal{U}^{(L)}\left(\left\{\lambda_{a}\right\}_{1}^{N} ;\left\{\mu_{a}\right\}_{1}^{N+1} ;\left\{y_{a}\right\}_{1}^{n+1} \mid \gamma\right)=\mathcal{U}\left(\left\{\mu_{i_{a}}\right\}_{1}^{n} ;\left\{y_{a}\right\}_{1}^{n+1} \mid \gamma\right)+\mathrm{O}\left(L^{-1}\right) .
$$

with a $\mathrm{O}$ that, again, is uniform and holomorphic in respect to $\mu_{i_{a}}$ or $y_{a}$ belonging to $U_{\delta / 2}$. It is also holomorphic in $\mathfrak{R}(\beta) \geq 0$.

By using the densification of the parameters $\lambda_{a}$ and $\mu_{a}$ on $[-q ; q]$, it is likewise easy to check that

$$
\operatorname{det}_{N+1}\left[\Xi^{(\mu)}[\xi]\right] \operatorname{det}_{N}\left[\Xi^{(\lambda)}\left[\xi_{\gamma \gamma^{(L)}}\right]\right]=\operatorname{det}^{2}[I-K / 2 \pi] \cdot\left(1+\mathrm{O}\left(L^{-1}\right)\right) .
$$

\section{$L \rightarrow+\infty$ limit of the remaining terms}

It is also readily seen due to the densification of the parameters $\lambda_{a}$ on $[-q ; q]$ that the sums over the discreet sets $\lambda_{i_{a}}$ can be replaced by integrals over $[-q ; q]$ up to $\mathrm{O}\left(L^{-1}\right)$ corrections. Finally, it remains to estimate the contributions of the functions $f^{(L)}$. If one focuses on the contributions of the integrals over $y_{a}, a=1, \ldots, n+1$ along the curves $\mathscr{C}_{\uparrow / \downarrow ; L}$ and $\mathscr{C}_{b d ; L}$, then one readily convinces oneself that one deals with the type of integrals studied in the proof of proposition B.1 Namely, these are precisely the integrals appearing when deriving the estimates for the functionals $I_{k}^{(L)}$ given in (B.6). Clearly, each of these integrals can be estimated successively. By repeating word for word the proof given in proposition B.1 one has that each of these integrals produces a $\mathrm{O}\left(L / w_{L}\right)=\mathrm{o}(1)$ contribution. Hence, this part of the contour $\mathscr{C}^{(L)}$ does not contribute to the thermodynamic limit.

As a consequence one obtains the following representation for $\rho_{N ; \mathrm{eff}}^{(m)}(x, t)$ :

$$
\begin{array}{r}
\rho_{N ; \text { eff }}^{(m)}(x, t)=\sum_{n=0}^{m} \frac{c(-1)^{n}}{n !} \frac{\partial^{m}}{\partial \gamma^{m}} \int_{-q}^{q} \mathrm{~d}^{n} \lambda \oint_{\mathscr{C}_{q}} \frac{\mathrm{d}^{n} z}{(2 i \pi)^{2 n}} \int_{\mathscr{C}} \frac{\mathrm{d}^{n+1} y}{(2 i \pi)^{n+1}} \frac{\mathrm{e}^{i x \mathcal{U}\left(\left\langle\lambda_{a}\right)_{1}^{n} ;\left\{y_{a}\right\}_{1}^{n+1} \mid \gamma\right)} \prod_{k=1}^{n+1} f\left(y_{k}, \gamma v\left(y_{k}\right)\right)}{\prod_{k=1}^{n}\left(z_{k}-\lambda_{k}\right)\left(y_{k}-z_{k}\right)\left(y_{n+1}-\lambda_{k}\right)} \operatorname{det}_{n}\left[\frac{y_{n+1}-z_{b}}{z_{a}-\lambda_{b}}\right] \\
\times \frac{\prod_{a=1}^{n} \prod_{b=1}^{n+1}\left(y_{b}-\lambda_{a}-i c\right)\left(\lambda_{a}-y_{b}-i c\right)}{\prod_{a, b=1}^{n+1}\left(y_{a}-y_{b}-i c\right) \prod_{a, b=1}^{n}\left(\lambda_{a}-\lambda_{b}-i c\right)} \prod_{k=1}^{n}\left\{4 \sin ^{2}\left[\pi \gamma v\left(\lambda_{k}\right)\right]\right\} \frac{\operatorname{det}_{n}\left[\delta_{k \ell}+\gamma \widehat{V}_{k \ell}[v]\right] \operatorname{det}_{n}\left[\delta_{k \ell}+\gamma \widehat{\bar{V}}_{k \ell}[v]\right]}{\operatorname{det}[I+\gamma R / 2 \pi] \operatorname{det}^{2}[I-K / 2 \pi]} \\
\times(1+\mathrm{o}(1))_{\mid \gamma=0} . \quad(\mathrm{C}
\end{array}
$$


In order to obtain the representation (C.1) for the thermodynamic limit it remains to drop the o (1) corrections.

We now provide an alternative representation for the thermodynamic limit $\rho_{\mathrm{ext}}^{(m)}(x, t)$.

Proposition C.1 The function $\rho_{\mathrm{ext}}^{(m)}(x, t)$ admits the representation

$$
\rho_{\mathrm{eff}}^{(m)}(x, t)=\lim _{w \rightarrow+\infty} \lim _{\beta \rightarrow 0} \lim _{s \rightarrow+\infty} \lim _{r \rightarrow+\infty}: \frac{\partial^{m}}{\partial \gamma^{m}}\left\{\widehat{E}_{-}^{2}(q) \mathrm{e}^{-\int_{-q}^{q}\left[i x u^{\prime}(\lambda)+\widehat{g}^{\prime}(\lambda)\right] \gamma v_{s}(\lambda) \mathrm{d} \lambda} X_{\mathscr{C}_{E}^{(w)}}\left[\gamma v_{s}, \widehat{E}_{-}^{-2}\right] \mathscr{G}_{\gamma ; 2 w}^{(\beta)}\left[\varpi_{r}\right]\right\}_{\mid \gamma=0}:
$$

The contour $\mathscr{C}_{E}^{(w)}=\mathscr{C}_{E}^{(\infty)} \cap\{z \in \mathbb{C}:|\mathfrak{R}(z)| \leq w\}$ corresponds to a compact approximation of $\mathscr{C}_{E}^{(\infty)}$ as depicted in Fig. 2. It is such that $\lim _{w \rightarrow+\infty} \mathscr{C}_{E}^{(w)}=\mathscr{C}_{E}^{(\infty)}$. The functional $X_{\mathscr{C}_{E}^{(w)}}$ has been defined in (B.34).

The functional $\mathscr{G}_{\gamma ; 2 w}^{(\beta)}\left[\varpi_{r}\right]$ appearing in (C.31) acts on the loop $\mathscr{C}\left(K_{2 w}\right)$ and has been defined in lemma A.1]

The compact approximation $\mathscr{C}_{E}^{(w)}$ of the contour $\mathscr{C}_{E}^{(\infty)}$ appearing in $(\mathbf{C} .31]$ is there to ensure the well-definiteness of the translation operators. Indeed, in the setting discussed in subsection 4.14 and appendix D the translation operators are, a priori, only defined for functionals that involve the values of their argument on some compact subset of $\mathbb{C}$. As a consequence, a priori, the $w \rightarrow+\infty$ limit and $r \rightarrow+\infty$ limit do not commute.

Also, the $\beta \rightarrow 0$ limit and the $w \rightarrow+\infty$ limits do not commute. These limit should be understood as follows. Given $w$ fixed and large enough, one considers the regular functional $\mathscr{G}_{\gamma ; 2 w}^{(\beta)}$ as introduced in lemma A.1 The value of $w$ defines an associated $\beta_{0} \in \mathbb{C}$ and $\widetilde{\gamma}_{0}>0$ such that $\mathscr{G}_{\gamma ; 2 w}^{(\beta)}$ is a regular functional for $\beta \in \widetilde{\boldsymbol{U}}_{\beta_{0}}$ and $|\gamma| \leq \gamma_{0}$ with a regularity constant large enough (in particular satisfying 4.30). These $\mathfrak{R}\left(\beta_{0}\right)$ and $\widetilde{\gamma}_{0}$ are such that $\mathfrak{R}\left(\beta_{0}\right) \rightarrow+\infty$ and $\widetilde{\gamma}_{0} \rightarrow 0$ when $w \rightarrow+\infty$.

Proof -

Let $\widetilde{E}_{-}^{-2}$ be as given in (4.51)-(4.52), $v_{s}$ as in (4.36) and $\varpi_{r}$ (4.32). In order to implement the operator substitution, we first expand the functional $X_{\mathscr{C}_{E}(w)}\left[v, \widetilde{E}_{-}^{2}\right]$ appearing in the $r h s$ of $(\bar{C} .31)$ into a series very similar to the one occurring in the proof of proposition B.4 The sole exception is that, this time, the sums over $\lambda_{i_{a}}$ 's are directly replaced by integrals over $[-q ; q]$ of the corresponding variables. Also, the function $f^{(L)}$ (resp. its associated contour $\mathscr{C}^{(L)}$ ) should be replaced by $f\left(\right.$ resp. $\mathscr{C}^{(w)}=\mathscr{C}_{E}^{(w)} \cup \widetilde{\mathscr{C}}_{q}$ ). At the end of the day, one deals with the multi-dimensional Lagrange series below

$$
\lim _{w \rightarrow+\infty} \lim _{s \rightarrow+\infty} \lim _{r \rightarrow+\infty} \sum_{n=0}^{m} i \frac{(-1)^{n}}{n !} \int_{-q}^{q} \frac{\mathrm{d}^{n} \lambda}{(2 i \pi)^{n}} \oint_{\mathscr{C}_{q}} \frac{\mathrm{d}^{n} z}{(2 i \pi)^{n}} \int_{\mathscr{\mathscr { C }}(w)} \frac{\mathrm{d}^{n+1} y}{(2 i \pi)^{n+1}} \widetilde{\mathcal{L}}_{\Gamma}^{(m)}\left[\mathcal{F} \mathscr{G}_{\gamma ; 2 w}^{(\beta)}\right] .
$$

The functional $\mathcal{F}$ appearing above reads

$$
\mathcal{F}\left[\gamma v_{s}\right]=\frac{\prod_{k=1}^{n+1} f\left(y_{k}, \gamma v_{s}\left(y_{k}\right)\right)}{\prod_{k=1}^{n}\left(z_{k}-\lambda_{k}\right)\left(y_{k}-z_{k}\right)} \prod_{k=1}^{n}\left(\frac{y_{n+1}-z_{k}}{y_{n+1}-\lambda_{k}}\right) \cdot \operatorname{det}_{n}\left[\frac{4 \sin ^{2}\left[\pi \gamma v_{s}\left(\lambda_{k}\right)\right]}{z_{a}-\lambda_{k}}\right] \mathrm{e}^{-i x \int_{-q}^{q} u^{\prime}(\lambda) \gamma v_{s}(\lambda) \mathrm{d} \lambda} \mathrm{e}^{-i x u(q)} \frac{\prod_{a=1}^{n} \mathrm{e}^{-i x u\left(\lambda_{a}\right)}}{\prod_{a=1}^{n+1} \mathrm{e}^{-i x u\left(y_{a}\right)}}
$$

And we have set

$$
\begin{aligned}
& \widetilde{\mathcal{L}}_{\Gamma}^{(m)}\left[\mathcal{F}_{\mathcal{G}^{(\beta) 2 w}}^{(\beta)}\right]=\sum_{\substack{n_{1}, \ldots, n_{s} \\
=0}}^{m} \prod_{p=1}^{s}\left\{\frac{\left[a_{p}\right]^{n_{p}}}{n_{p} !}\right\} \frac{\partial^{m}}{\partial \gamma^{m}}\left\{\frac{\prod_{k=1}^{n+1} \mathrm{e}^{\widetilde{g}_{1, s}\left(y_{k}\right)}}{\mathrm{e}^{\widetilde{g}_{1, s}(q)} \prod_{k=1}^{n} \mathrm{e}^{\widetilde{g}_{1, s}\left(\lambda_{k}\right)}} \prod_{p=1}^{s}\left\{\Gamma\left[\gamma v_{s}\right]\left(t_{p}\right)\right\}^{n_{p}}\right. \\
& \left.\times \frac{\prod_{k=1}^{n+1} \mathrm{e}^{\widetilde{g}_{1, s}\left(y_{k}\right)}}{\mathrm{e}^{\widetilde{g}_{1, s}(q)} \prod_{k=1}^{n} \mathrm{e}^{\widetilde{g}_{1, s}\left(\lambda_{k}\right)}} \mathrm{e}^{-\int_{-q}^{q} \widetilde{g}^{\prime}(\lambda) \gamma v_{s}(\lambda) \mathrm{d} \lambda} \cdot \mathcal{F}\left[\gamma v_{s}\right]_{\left.\right|_{\gamma_{a}=0} ^{\gamma=0}} \mathscr{G}_{\gamma ; 2 w}^{(\beta)}\left[\varpi_{r}\right]\right\}_{\mid \gamma=0} .
\end{aligned}
$$


The functional $\Gamma$ is evaluated at the discretization points $t_{p}, p=1, \ldots, s$ for the contour $\mathscr{C}_{\text {out }}$ appearing in the lhs of Fig. 1,

One can implement the operator substitution on the level of (C.33) as it was done in the proof of theorem C.1. The well-foundedness of these manipulations (in particular the justification of the exchange of various limits, partial derivatives and integrals over compact contours) is justified along very similar lines. Once upon taking the $r \rightarrow+\infty$ limit we end-up with the below multidimensional Lagrange series

$$
\mathcal{L}_{\Gamma}^{(m)}\left[\mathcal{F} \mathscr{G}_{\gamma ; 2 w}^{(\beta)}\right]=\sum_{\substack{n_{1}, \ldots, n_{s} \\=0}}^{m} \prod_{p=1}^{s}\left\{\frac{1}{n_{p} !} \frac{\partial^{n_{p}}}{\partial \varsigma_{p}^{n_{p}}}\right\} \frac{\partial^{m}}{\partial \gamma^{m}}\left\{\prod_{p=1}^{s}\left\{\Gamma\left[\gamma \widetilde{\gamma}_{s}\right]\left(t_{p}\right)\right\}^{n_{p}} \cdot \mathcal{F}\left[\gamma \widetilde{v}_{s}\right]_{\substack{\gamma=0 \\ S_{a}=0}} \mathscr{G}_{\gamma ; 2 w}^{(\beta)}[\varpi]\right\}_{\mid \gamma=0} .
$$

There, we agree upon

$$
\widetilde{v}_{s}\left(\lambda ;\left\{\varsigma_{a}\right\}\right)=v_{s}\left(\lambda ;\left\{\varsigma_{a}\right\}\right)+\sum_{b=1}^{s} \frac{t_{b+1}-t_{b}}{2 i \pi\left(t_{b}-\lambda\right)}\left\{\phi\left(t_{b}, q\right)+\sum_{a=1}^{n} \phi\left(t_{b}, \lambda_{a}\right)-\sum_{a=1}^{n+1} \phi\left(t_{b}, y_{a}\right)\right\} .
$$

Also, the function $\varpi$ is to be considered as a functional of $\widetilde{v}_{s}$

$$
\varpi\left[\widetilde{v}_{s}\right](\lambda)=\sum_{k=1}^{n+1} \frac{1}{\lambda-y_{k}}-\frac{1}{\lambda-q}-\sum_{k=1}^{n} \frac{1}{\lambda-\lambda_{k}}-\int_{-q}^{q} \frac{\gamma \widetilde{v}_{s}(\tau)}{(\tau-\lambda)^{2}} \mathrm{~d} \tau .
$$

The multidimensional Lagrange series (C.34) has been studied in appendix D.5.1 Its $s \rightarrow+\infty$ limit is uniform in respect to the auxiliary parameters $\left\{\lambda_{a}\right\}_{1}^{n},\left\{z_{a}\right\}_{1}^{n}$ and $\left\{y_{a}\right\}_{1}^{n+1}$. Hence, just as in the proof of theorem D.1, one is allowed to exchange the $s \rightarrow+\infty$ limit with the integration over the compact contours. One can then apply the results of appendix D.5.1 leading to

$$
\lim _{s \rightarrow+\infty} \mathcal{L}_{\Gamma}^{(m)}\left[\mathcal{F} \mathscr{G}_{\gamma ; 2 w}^{(\beta)}\right]=\frac{\partial^{m}}{\partial \gamma^{m}}\left\{\frac{\mathcal{F}[\gamma v] \mathscr{G}_{\gamma ; 2 w}^{(\beta)}[\varpi[v]]}{\operatorname{det}_{[-q ; q]}[I+\gamma R / 2 \pi]}\right\}_{\mid \gamma=0}
$$

The function $v$ appearing above is the unique solution to the linear integral equation (C.3). One can build on this result so as to simplify the obtained expression. The expression for the functional function $\mathscr{G}_{\gamma ; 2 w}^{(\beta)}[\varpi[v]]$ is simplified with the help of lemma A.3.

By using the linear integral equation satisfied by $v$ together with the representation of $u$ in terms of $\phi$ and $u_{0}$ (2.14), we get that the oscillating factor present in $\mathcal{F}[\gamma v]$ coincides with the one appearing in theorem C.1

$$
\sum_{a=1}^{n+1} u\left(y_{a}\right)-\sum_{a=1}^{n} u\left(\lambda_{a}\right)-u(q)-\gamma \int_{-q}^{q} u^{\prime}(\lambda) v(\lambda) \mathrm{d} \lambda=\mathcal{U}\left(\left\{\lambda_{a}\right\}_{1}^{n},\left\{y_{a}\right\}_{1}^{n+1} \mid \gamma\right)-2 i \beta p_{F} .
$$

We are thus led to the below representation for the rhs of (C.31)

$$
\begin{aligned}
\lim _{w \rightarrow+\infty} & \lim _{\beta \rightarrow 0} \mathrm{e}^{2 x \beta p_{F}} \sum_{n=0}^{m} \frac{c(-1)^{n}}{n !} \frac{\partial^{m}}{\partial \gamma^{m}} \int_{-q}^{q} \mathrm{~d}^{n} \lambda \oint_{\mathscr{C}_{q}} \frac{\mathrm{d}^{n} z}{(2 i \pi)^{2 n}} \int_{\mathscr{C}(w)} \frac{\mathrm{d}^{n+1} y}{(2 i \pi)^{n+1}} \frac{\mathrm{e}^{i x \mathcal{U}\left(\left\{\lambda_{a}\right\}_{1}^{n} ;\left\{y_{a} y_{1}^{n+1} \mid \gamma\right)\right.} \prod_{k=1}^{n+1} f\left(y_{k}, \gamma v\left(y_{k}\right)\right)}{\prod_{k=1}^{n}\left(z_{k}-\lambda_{k}\right)\left(y_{k}-z_{k}\right)\left(y_{n+1}-\lambda_{i_{k}}\right)} \operatorname{det}_{n}\left[\frac{y_{n+1}-z_{b}}{z_{a}-\lambda_{b}}\right] \\
& \times \frac{\prod_{a=1}^{n} \prod_{b=1}^{n+1}\left(y_{b}-\lambda_{a}-i c\right)\left(\lambda_{a}-y_{b}-i c\right)}{\prod_{a, b=1}^{n+1}\left(y_{a}-y_{b}-i c\right) \prod_{a, b=1}^{n}\left(\lambda_{a}-\lambda_{b}-i c\right)} \prod_{k=1}^{n}\left[4 \sin ^{2}\left[\pi \gamma v\left(\lambda_{k}\right)\right]\right] \frac{\operatorname{det}_{n}\left[\delta_{k \ell}+\gamma \widehat{V}_{k \ell}[v]\right] \operatorname{det}_{n}\left[\delta_{k \ell}+\gamma \widehat{\bar{V}}_{k \ell}[v]\right]}{\operatorname{det}[I+\gamma R / 2 \pi] \operatorname{det}^{2}[I-K / 2 \pi]} .
\end{aligned}
$$


Tha auxiliary arguments of the entries $\widehat{V}_{k \ell}[v]$ and $\widehat{\bar{V}}_{k \ell}[v]$ are undercurrent by those of $v$.

One can carry out the analytic continuation from $\beta \in \widetilde{\boldsymbol{U}}_{\beta_{0}}$ up to $\beta=0$ as the potential singularities of the two determinants are canceled by the pre-factors $\prod_{k=1}^{n} \sin ^{2}\left[\pi \gamma v\left(\lambda_{k}\right)\right]$.

There is no problem to take the $w \rightarrow+\infty$ limit of the above integrals. Indeed $\mathscr{C}_{E}^{(\infty)}$ is chosen in such a way that $\mathrm{e}^{i x u\left(y_{a}\right)}, a=1, \ldots, n+1$ is decaying exponentially fast in $y_{a}$ when $y_{a} \rightarrow \infty$ along $\mathscr{C}_{E}^{(\infty)}$. As the rest of the integrand is a $\mathrm{O}\left(y_{a}^{n}\right), a=1, \ldots, n+1$ at infinity, the integrals along $\mathscr{C}_{E}^{(\infty)}$ are convergent. Once upon taking the $\beta \rightarrow 0$ and the $w \rightarrow+\infty$ limits, we recover the representation given in (C.1).

\section{Functional Translation operator}

In this appendix, we build a convenient for our purposes representation of a functional translation. Our representation applies to sufficiently regular classes of functionals acting on holomorphic functions. Our construction utilizes multidimensional Lagrange series (see eg. [1]).

\section{D.1 Lagrange series}

Theorem D.1 [1]

Let $\mathcal{D}_{0, r}=\{z \in \mathbb{C}:|z|<r\}$. Assume that

- $\varphi_{j}\left(\left\{\varsigma_{a}\right\}_{1}^{s}\right), j=1, \ldots$, s and $f\left(\left\{\varsigma_{a}\right\}_{1}^{s}\right)$ are holomorphic functions of $\left\{\varsigma_{a}\right\}_{1}^{s}$ belonging to the Cartesian product $\mathcal{D}_{0, r}^{s}$

- there exists a series of radii $r_{j}<r$ such that for $\left|\varsigma_{j}\right|=r_{j}, j=1, \ldots$, , one has $\left|\varphi_{j}\left(\left\{\varsigma_{a}\right\}\right)\right|<r_{j}$.

Then, the multidimensional Lagrange series

$$
\mathcal{L}_{s}=\left.\sum_{\substack{n_{1}, \ldots, n_{s} \\ \in \in \mathbb{N}}} \prod_{r=1}^{s}\left\{\frac{1}{n_{r} !} \frac{\partial^{n_{r}}}{\partial \varsigma_{r}^{n_{r}}}\right\} \cdot \prod_{r=1}^{s} \varphi_{r}^{n_{r}}\left(\left\{\boldsymbol{S}_{a}\right\}\right) \cdot f\left(\left\{\boldsymbol{S}_{a}\right\}\right)\right|_{\varsigma_{p}=0}
$$

is convergent and its sum is given by

$$
\mathcal{L}_{s}=\frac{f\left(\left\{z_{a}\right\}\right)}{\operatorname{det}_{s}\left[\delta_{j k}-\frac{\partial}{\partial \varsigma_{k}} \varphi_{j}\left(\left\{\varsigma_{a}\right\}\right)\right]_{\left\{\varsigma_{a}\right\}=\left\{z_{a}\right\}}} .
$$

Above, $\left(z_{1}, \ldots, z_{s}\right)$ stands for the unique solution to the system $z_{j}=\varphi_{j}\left(\left\{z_{a}\right\}\right)$ such that $\left|z_{j}\right|<r$ for all $j$. The uniqueness and existence of this solution is part of the conclusion of this theorem.

\section{D.2 Some preliminary definitions}

Throughout this appendix, $M$ and $K$ will always stand for two compacts of $\mathbb{C}$ such that $K \subset \operatorname{Int}(M), M$ has $n$ holes (ie $\mathbb{C} \subset M$ has $n$ bounded connected components) and $\partial M$ can be realized as disjoint union of $n+1$ smooth Jordan curves $\gamma_{a}:[0 ; 1] \rightarrow \partial M=\amalg_{a=1}^{n+1} \gamma_{a}([0 ; 1])$.

\footnotetext{
${ }^{\dagger}$ we remind that $\gamma_{a}$ satisfies $\gamma_{a}(0)=\gamma_{a}(1)$ and $\gamma_{a \mid[0 ; 1[}$ is injective.
} 
Let $h$ be a holomorphic function on $M$ and set

$$
f_{s}\left(\lambda \mid\left\{\varsigma_{a, p}\right\}\right)=\sum_{p=1}^{s} \sum_{a=1}^{n+1} \frac{\left(t_{a, p+1}-t_{a, p}\right)}{2 i \pi\left(t_{a, p}-\lambda\right)} \varsigma_{a, p}+\sum_{p=1}^{s} \sum_{a=1}^{n+1} \frac{\left(t_{a, p+1}-t_{a, p}\right)}{2 i \pi\left(t_{a, p}-\lambda\right)} h\left(t_{a, p}\right),
$$

The points $t_{a, p}$ correspond to the discretization points for $\partial M$ associated with the Jordan curves $\gamma_{a}$, as given in definition 4.2. It follows readily that the function $\lambda \mapsto f_{s}\left(\lambda \mid\left\{\varsigma_{a}\right\}\right)$ is holomorphic in $\lambda \in K$. Moreover, given any holomorphic function $v(\lambda, \boldsymbol{y}) \in \mathscr{O}\left(M \times W_{y}\right)$ where $W_{y}$ is a compact in $\mathbb{C}^{\ell_{y}}, \ell_{y} \in \mathbb{N}$, one has that

$$
f_{s}\left(\lambda \mid\left\{v\left(t_{a, p}, \boldsymbol{y}\right)\right\}\right) \underset{s \rightarrow+\infty}{\longrightarrow} \int_{\partial M} \frac{v(\zeta, \boldsymbol{y})+h(\zeta)}{2 i \pi(\zeta-\lambda)} \mathrm{d} \zeta=v(\lambda, \boldsymbol{y})+h(\lambda) \quad \text { uniformly in } \lambda \in K \text { and } \boldsymbol{y} \in W_{y} .
$$

This convergence holds since $(\zeta, \lambda, \boldsymbol{y}) \mapsto v(\zeta, \boldsymbol{y})+h(\zeta) /(\zeta-\lambda)$ is uniformly continuous on $\partial M \times K \times W_{y}$.

We recall that, given a holomorphic function $h$ on $M$ (and hence also on some open neighborhood of $M$ ), and $S$ a subset of $M$, we denote $\|h\|_{S}=\sup _{s \in S}|h(s)|$.

\section{D.3 Pure translations}

We are now in position to establish a representation for translation operators for functionals acting on holomorphic functions.

Proposition D.1 Let $\mathcal{F}[\cdot](z), z \in W_{z} \subset \mathbb{C}^{\ell_{z}}$ be a regular functional in respect to the pair $(M, K)$ and let the functions $f_{s}, v$ and $h$ and the compacts $M$ and $K$ be defined as above. Then, for any $\left(m, k_{1}, \ldots, k_{\ell_{y}}\right) \in \mathbb{N}^{\ell_{y}+1}$

$$
\left.\lim _{s \rightarrow+\infty} \prod_{j=1}^{\ell_{y}} \frac{\partial^{k_{j}}}{\partial y_{j}^{k_{j}}} \cdot \prod_{p=1}^{s} \prod_{a=1}^{n+1} \mathrm{e}^{v\left(t_{a, p}, \boldsymbol{y}\right) \partial_{\zeta a, p}} \cdot \frac{\partial^{m}}{\partial \gamma^{m}} \mathcal{F}\left[\gamma f_{s}\left(* \mid\left\{\varsigma_{a, p}\right\}\right)\right](z)\right|_{\varsigma_{a, p}=0}=\prod_{j=1}^{\ell_{y}} \frac{\partial^{k_{j}}}{\partial y_{j}^{k_{j}}} \frac{\partial^{m}}{\partial \gamma^{m}} \mathcal{F}[\gamma \nu(*, \boldsymbol{y})+\gamma h(*)](z) .
$$

Above, the $\cdot$ inside of the argument of indicates the running variable on which the functional $\mathcal{F}[\cdot](y)$ acts. This convergence holds uniformly in $(\gamma, \boldsymbol{y}, z)$ belonging to compact subsets of $\mathcal{D}_{0, \gamma_{0}} \times \operatorname{Int}\left(W_{y}\right) \times \operatorname{Int}\left(W_{z}\right)$, where

$$
3 \gamma_{0}=\frac{C_{\mathcal{F}}}{2\|v\|_{M \times W_{y}}+\|h\|_{M}} \frac{\pi \mathrm{d}(\partial M, K)}{|\partial M|+2 \pi \mathrm{d}(\partial M, K)},
$$

$|\partial M|$ stands for the length of $\partial M, \mathrm{~d}(\partial M, K)$ for the distance of $K$ to $\partial M$ and $C_{\mathcal{F}}>0$ is the constant of regularity of $\mathcal{F}$. Finally, $\mathcal{D}_{0, \gamma_{0}}=\left\{z \in \mathbb{C}:|z| \leq \gamma_{0}\right\}$.

Proof - We first consider the case $m=0$ and $k_{1}=\cdots=k_{\ell}=0$. We assume that $s$ is taken large enough so that

$$
\sum_{p=1}^{s} \sum_{a=1}^{n+1}\left|t_{a, p}-t_{a, p+1}\right| \leq 2|\partial M|
$$

Then, for $|\gamma|<2 \gamma_{0}$ and $\left|s_{a}\right| \leq 2\|v\|_{M}$, one has

$$
\left|\gamma f_{s}\left(\lambda \mid\left\{\varsigma_{a, p}\right\}\right)\right| \leq|\gamma|\left(\sup _{a, p}\left|\varsigma_{a, p}\right|+\|h\|_{M}\right) \times \sum_{p=1}^{s} \sum_{a=1}^{n+1} \frac{\left|t_{a, p+1}-t_{a, p}\right|}{2 \pi\left|\lambda-t_{a, p}\right|} \leq|\gamma|\left(\sup _{a}\left|\varsigma_{a, p}\right|+\|h\|_{M}\right) \frac{2|\partial M|}{2 \pi \mathrm{d}(\partial M, K)}<\frac{2}{3} C_{\mathcal{F}},
$$


Hence,

$$
\left(\gamma,\left\{\varsigma_{a, p}\right\}, z\right) \mapsto \mathcal{F}\left[\gamma f_{s}\left(* \mid\left\{\varsigma_{a, p}\right\}\right)\right](z) \quad \text { is holomorphic in } \quad\left(\gamma,\left\{\varsigma_{a, p}\right\}, z\right) \in \mathcal{D}_{0,2 \gamma_{0}} \times \mathcal{D}_{0,2\|v\|_{M}}^{s(n+1)} \times W_{z},
$$

this for any $s$ large enough. As a consequence, the below multi-dimensional Taylor series is convergent uniformly in $(\gamma, \boldsymbol{y}, \boldsymbol{z}) \in \mathcal{D}_{0,2 \gamma_{0}} \times W_{y} \times W_{z}$ and

$$
\begin{aligned}
& \left.\prod_{p=1}^{s} \prod_{a=1}^{n+1} \mathrm{e}^{v\left(t_{a, p}, \boldsymbol{y}\right) \partial_{s a, p}} \mathcal{F}\left[\gamma f_{s}\left(* \mid\left\{\varsigma_{a, p}\right\}\right)\right](\boldsymbol{z})\right|_{S_{a, p}=0} \\
& \left.\quad \equiv \sum_{n_{a, p} \geq 0}^{+\infty} \prod_{p=1}^{s} \prod_{a=1}^{n+1}\left\{\frac{\left[v\left(t_{a, p}, \boldsymbol{y}\right)\right]^{n_{a, p}}}{\left(n_{a, p}\right) !} \frac{\partial^{n_{a, p}}}{\partial \varsigma_{a, p}}\right\} \cdot \mathcal{F}\left[\gamma f_{s}\left(* \mid\left\{\varsigma_{a, p}\right\}\right)\right](z)\right|_{\varsigma_{a, p}=0}=\mathcal{F}\left[\gamma f_{s}\left(* \mid\left\{v\left(t_{a, p}, \boldsymbol{y}\right)\right\}\right)\right](\boldsymbol{z}) .
\end{aligned}
$$

Moreover, for any $\boldsymbol{y} \in W_{y}$ and $\gamma \in \overline{\mathcal{D}}_{0,2 \gamma_{0}}$, one has the bound $\left\|\gamma f_{s}\left(* \mid\left\{v\left(t_{a, p}, \boldsymbol{y}\right)\right\}\right)\right\|_{K}+|\gamma|\left(\|v(\cdot, \boldsymbol{y})\|_{K}+\|h\|_{K}\right)<C_{\mathcal{F}}$. As a consequence, by 4.14

$$
\begin{aligned}
\| \mathcal{F}\left[\gamma f_{s}\left(* \mid\left\{v\left(t_{a, p}, \boldsymbol{y}\right)\right\}\right)\right](\boldsymbol{z})-\mathcal{F}[\gamma v(*, \boldsymbol{y})+ & \gamma h(*)](\boldsymbol{z}) \|_{\overline{\mathcal{D}}_{0,2 \gamma_{0}} \times W_{y} \times W_{z}} \\
& \leq \gamma_{0} C^{\prime}\left\|f_{s}\left(\lambda \mid\left\{v\left(t_{a, p}, \boldsymbol{y}\right)\right\}\right)-v(\lambda, \boldsymbol{y})-h(\lambda)\right\|_{K \times W_{y}} \underset{s \rightarrow+\infty}{\longrightarrow} 0,
\end{aligned}
$$

due to (D.3). The norm in the first line is computed in respect to $(\gamma, \boldsymbol{y}, z) \in \overline{\mathcal{D}}_{0,2 \gamma_{0}} \times W_{y} \times W_{z}$. The one in the second line in respect to $(\lambda, \boldsymbol{y}) \in K \times W_{y}$. We insisted explicitly on the variable-dependence of the functions so as to make this fact clear.

It remains to show that the convergence also holds uniformly on all compacts of $\mathcal{D}_{0,2 \gamma_{0}} \times \operatorname{Int}\left(W_{y}\right) \times \operatorname{Int}\left(W_{z}\right)$ when considering partial derivatives in respect to $\gamma, y_{1}, \ldots, y_{\ell_{y}}$ of finite total order.

One can exchange any such partial derivatives with the Taylor series in (D.8) in as much as its partial sums define a sequence of holomorphic functions that is uniformly convergent on $\overline{\mathcal{D}}_{0,2 \gamma_{0}} \times W_{y} \times W_{z}$. The same arguments can be applied to the sequence of holomorphic functions $\mathcal{F}\left[\gamma f_{s}\left(* \mid\left\{v\left(t_{a, p}, \boldsymbol{y}\right)\right\}\right)\right](z)$.

Corollary D.1 Assume that the conditions and notations of proposition D.1 hold. Let $\mathscr{C}^{\left(\ell_{y}\right)}=\mathscr{C}_{1} \times \cdots \times \mathscr{C}_{\ell_{y}}$ and $\widetilde{\mathscr{C}}^{\left(\ell_{z}\right)}=\widetilde{\mathscr{C}}_{1} \times \cdots \times \widetilde{\mathscr{C}}_{\ell_{z}}$ be Cartesian products of compact curves in $\mathbb{C}$ such that $\mathscr{C}^{\left(\ell_{y}\right)} \subset \operatorname{Int}\left(W_{y}\right)$ and $\widetilde{\mathscr{C}}^{\left(\ell_{z}\right)} \subset \operatorname{Int}\left(W_{z}\right)$.

Then one has

$$
\begin{aligned}
& \left.\lim _{s \rightarrow+\infty} \sum_{n_{a, p}=0}^{+\infty} \prod_{p=1}^{s} \prod_{a=1}^{n+1}\left\{\frac{1}{\left(n_{a, p}\right) !} \frac{\partial^{n_{a, p}}}{\partial \varsigma_{a, p}^{n_{a, p}}}\right\} \cdot \int_{\mathscr{C}\left(\ell_{y}\right)} \mathrm{d}^{\ell_{y}} \boldsymbol{y} \int_{\mathscr{\mathscr { C }}\left(\ell_{z}\right)} \mathrm{d}^{\ell_{z}} z \prod_{j=1}^{\ell_{y}} \frac{\partial^{k_{j}}}{\partial y_{j}^{k_{j}}} \cdot \prod_{p=1}^{s} \prod_{a=1}^{n+1}\left[v\left(t_{a, p}, \boldsymbol{y}\right)\right]^{n_{a, p}} \frac{\partial^{m}}{\partial \gamma^{m}} \mathcal{F}\left[\gamma f_{s}\left(* \mid\left\{\varsigma_{a, p}\right\}\right)\right](\boldsymbol{y}, \boldsymbol{z})\right|_{\varsigma_{a, p}=0} \\
& =\int_{\mathscr{C}\left(\ell_{y}\right)} \mathrm{d}^{\ell_{y}} \boldsymbol{y} \int_{\widetilde{\mathscr{C}}\left(\ell_{z}\right)} \mathrm{d}^{\ell_{z}} \boldsymbol{z} \prod_{j=1}^{\ell_{y}} \frac{\partial^{k_{j}}}{\partial y_{j}^{k_{j}}} \frac{\partial^{m}}{\partial \gamma^{m}} \mathcal{F}[\gamma v(*, \boldsymbol{y})+\gamma h(*)](\boldsymbol{y}, \boldsymbol{z}) .
\end{aligned}
$$

this uniformly in $\gamma$ belonging to compact subsets of $\mathcal{D}_{0, \gamma_{0}}$.

Note that it $\mathcal{F}$ depends on a third set of variables belonging to a compact, the results hold as well in respect to this third set uniformly on the compact.

Proof- 
Proposition D.1 allows one to conclude, in virtue of the uniform convergence of the sequences, that for $\gamma$ belonging to compact subsets of $\mathcal{D}_{0, \gamma_{0}}$ one has the equality

$$
\begin{aligned}
\left.\lim _{s \rightarrow+\infty} \sum_{n_{a, p}=0}^{+\infty} \int_{\mathscr{C}\left(\ell_{y}\right)} \mathrm{d}^{\ell_{y}} \boldsymbol{y} \int_{\tilde{\mathscr{C}}\left(\ell_{z}\right)} \mathrm{d}^{\ell_{z}} \boldsymbol{z} \prod_{p=1}^{s} \prod_{a=1}^{n+1}\left\{\frac{1}{n_{a, p} !} \frac{\partial^{n_{a, p}}}{\partial \varsigma_{a, p}^{n_{a, p}}}\right\} \cdot \prod_{j=1}^{\ell_{y}} \frac{\partial^{k_{j}}}{\partial y_{j}^{k_{j}}} \cdot \prod_{p=1}^{s} \prod_{a=1}^{n+1}\left[v\left(t_{a, p}, \boldsymbol{y}\right)\right]^{n_{a, p}} \frac{\partial^{m}}{\partial \gamma^{m}} \mathcal{F}\left[\gamma f_{s}\left(* \mid\left\{\varsigma_{a, p}\right\}\right)\right](\boldsymbol{y}, \boldsymbol{z})\right|_{S_{a, p}=0} \\
=\int_{\mathscr{C}^{\left(\ell_{y}\right)}} \mathrm{d}^{\ell_{y}} \boldsymbol{y} \int_{\widetilde{\mathscr{C}}\left(\ell_{z}\right)} \mathrm{d}^{\ell_{z}} z \prod_{j=1}^{\ell_{y}} \frac{\partial^{k_{j}}}{\partial y_{j}^{k_{j}}} \frac{\partial^{m}}{\partial \gamma^{m}} \mathcal{F}[\gamma v(*, \boldsymbol{y})+\gamma h(*)](\boldsymbol{y}, \boldsymbol{z}) \text {. (D.10) }
\end{aligned}
$$

The integrals occurring in the first line of $(\overline{D .10})$ are over compact curves and the integrand is smooth in respect to the integration variables $(\boldsymbol{y}, z)$ and the auxiliary parameters $\varsigma_{a, p}$. As a consequence, the partial $\varsigma_{a, p}$-derivatives can be pulled outside of the integration symbols.

\section{D.4 Weighted translation}

One can generalize the notion of functional translation with the help of multi-dimensional Lagrange series and consider more complex objects. For this purpose, we need to introduce some more definitions. Also, from now on we only focus on the case of a compact $M$ without holes.

Let $\Gamma[\cdot](\mu)$ be a one parameter family of functionals such that:

- There exists a constant $C_{\Gamma}>0$ such that if $v(\lambda, \boldsymbol{y})$ is holomorphic in $(\lambda, \boldsymbol{y}) \in M \times W_{y}$, with $W_{y} \subset \mathbb{C}^{\ell_{y}}$ and $\|v\|_{K \times W_{y}}<C_{\Gamma}$ then $(\lambda, \boldsymbol{y}) \mapsto \Gamma[v(*, \boldsymbol{y})](\lambda)$ is holomorphic in $M \times W_{y}$.

- There exists a contour $\mathscr{C}$ in $\operatorname{Int}(K)$ such that for $\|\rho\|_{K}+\|\tau\|_{K} \leq C_{\Gamma}$ one has

$$
\Gamma[\rho](\mu)-\Gamma[\tau](\mu)=\int_{\mathscr{C}}(\rho-\tau)(\zeta) \frac{\delta \Gamma[v]}{\delta v(\zeta)}(\mu)_{\mid v=\tau} \mathrm{d} \zeta+\mathrm{o}\left(\|\rho-\tau\|_{K}\right) .
$$

$\delta \Gamma[v](\mu) / \delta v(\zeta)$ will be called the functional derivative of $\Gamma$. This functional derivative is such that, for any $\tau$ holomorphic on $M$ with $\|\tau\|_{K}<C_{\Gamma}$, there exists an open neighborhood $\mathcal{V}(\mathscr{C})$ of the contour $\mathscr{C}$ in (D.11) such that

$$
\left.(\mu, \zeta) \mapsto \frac{\delta \Gamma[v](\mu)}{\delta v(\zeta)}\right|_{\nu=\tau} \quad \text { is holomorphic in }(\mu, \zeta) \in M \times \mathcal{V}(\mathscr{C})
$$

- There exists a constant $C_{\Gamma}^{\prime}>0$ such that for $\|\tau\|_{K}+\|v\|_{K} \leq C_{\Gamma}$ one has

$$
\|\Gamma[v](\mu)\|_{M} \leq C_{\Gamma}^{\prime}\|v\|_{K} \quad \text { and } \quad\|\Gamma[\tau](\mu)-\Gamma[v](\mu)\|_{M} \leq C_{\Gamma}^{\prime}\|v-\tau\|_{K} .
$$

The properties of the functional $\Gamma[\cdot](\lambda)$ ensure the solvability of an associated integral equation

Lemma D.1 Let the compacts $M, K$ and the one parameter family of functional $\Gamma[\cdot](\lambda)$ be as defined above. Let $h \in \mathscr{O}(M)$ and $r, \gamma_{0}$ be such that

$$
2 \gamma_{0}\left(r+\|h\|_{M}\right)\left(1+\frac{2|\partial M|}{2 \pi \mathrm{d}(\partial M, K)}\right) \leq \frac{r \min \left(1, C_{\Gamma}\right)}{2\left(r+C_{\Gamma}^{\prime}\right)}, \quad \text { and } \quad 2 C_{\Gamma}^{\prime} \gamma_{0}\left(1+\frac{2|\partial M|}{2 \pi \mathrm{d}(\partial M, K)}\right)<\frac{\min \left(1, C_{\Gamma}\right)}{2}
$$

Then for $|\gamma| \leq \gamma_{0}$, there exists a unique solution $\rho$ to the equation $\rho(\lambda)=\Gamma[\gamma \rho(\cdot)+\gamma h(\cdot)](\lambda)$. This solution is holomorphic in $(\lambda, \gamma) \in M \times \overline{\mathcal{D}}_{0, \gamma_{0}}$ and such that $\|\rho\|_{M}<r$. 
Proof-

Suppose that $\rho$ and $\rho^{\prime}$ are two solutions. Then for $|\gamma|<2 \gamma_{0}$ one has, by construction of $\gamma_{0}$, that $|\gamma|\|\rho+h\|_{K}+$ $|\gamma|\left\|\rho^{\prime}+h\right\|_{K}<C_{\Gamma}$. As a consequence,

$$
\left\|\rho-\rho^{\prime}\right\|_{M}=\left\|\Gamma[\gamma(\rho+h)]-\Gamma\left[\gamma\left(\rho^{\prime}+h\right)\right]\right\|_{M} \leq 2 C_{\Gamma}^{\prime} \gamma_{0}\left\|\rho-\rho^{\prime}\right\|_{M}<\left\|\rho-\rho^{\prime}\right\|_{M} .
$$

Therefore, $\rho=\rho^{\prime}$ on $M$, this uniformly in $|\gamma| \leq 2 \gamma_{0}$.

In order to prove the existence, one considers the sequence of holomorphic functions on $M: h_{0}=h$ and, for $n \geq 1 h_{n}(\lambda)=h(\lambda)+\Gamma\left[\gamma h_{n-1}(\cdot)\right](\lambda)$. It is readily seen by straightforward induction that, for all $n \in \mathbb{N}$ and $|\gamma| \leq \gamma_{0}$,

$$
\gamma_{0}\left\|h_{n}\right\|_{K} \leq C_{\Gamma} / 2 \quad \text { and } \quad\left\|h_{n+1}-h_{n}\right\|_{M} \leq\left\|h_{n}-h_{n-1}\right\|_{M} / 2
$$

Hence $h_{n}$ is a Cauchy sequence in the space of holomorphic functions on $\operatorname{Int}(K)$. It is thus convergent to some holomorphic function $\widetilde{h}$ on Int $(M)$. Since $\widetilde{h}(\lambda)=h(\lambda)+\Gamma[\gamma \widetilde{h}](\lambda)$, it can be analytically continued to a holomorphic function on $M$. Then, the function $\rho=\widetilde{h}-h$ solves $\rho(\lambda)=\Gamma[\gamma(\rho+h)](\lambda)$. It also follows that then $\|\rho\|_{M}<r$

Proposition D.2 Let $f_{s}$ be as in (D.2) and assume that the functional $\Gamma[\rho](\mu)$ satisfies to the assumptions given above. Let $\mathcal{F}[\cdot](z)$, with $z \in W_{z} \subset \mathbb{C}^{\ell_{z}}$, be a regular functional in the sense of definition 4.1 Set

$$
\mathcal{L}_{\Gamma}(\gamma, z)=\lim _{s \rightarrow+\infty}:\left.\prod_{r=1}^{s} \mathrm{e}^{\left.\Gamma\left[\gamma f_{s}\left(* \mid \varsigma_{p}\right\}\right)\right]\left(t_{r}\right) \partial_{S r}} \mathcal{F}\left[\gamma f_{s}\left(* \mid\left\{\varsigma_{p}\right\}\right)\right](z)\right|_{S_{p}=0}:,
$$

where : · indicates that the expression is ordered in such a way that all the partial derivatives appear to the left (cf. subsection 4.3) and $t_{r}$ are the discretization points of $\partial M$.

Then there exists $\gamma_{0}>0$ such that $\mathcal{L}_{\Gamma}(\gamma, z)$ defines a holomorphic function of $(\gamma, z) \in \overline{\mathcal{D}}_{0, \gamma_{0}} \times \operatorname{Int}\left(W_{z}\right)$. The convergence of the rhs of (D.17) to $\mathcal{L}_{\Gamma}(\gamma, z)$ is uniform on compact subsets of $\overline{\mathcal{D}}_{0, \gamma_{0}} \times \operatorname{Int}\left(W_{z}\right)$, and this in respect to any partial $\gamma$ or $z$-derivative of finite order. $\mathcal{L}_{\Gamma}(\gamma, z)$ is given by

$$
\mathcal{L}_{\Gamma}(\gamma, z)=\frac{\mathcal{F}[\gamma \rho](z)}{\operatorname{det} \mathscr{C}_{\mathscr{C}}\left[I-\gamma \frac{\delta \Gamma[v]}{\delta v(\mu)}(\lambda)\right]_{\mid v=\gamma \rho}} \text { with } \rho \text { being the unique solution to } \rho(\lambda)=h(\lambda)+\Gamma[\gamma \rho](\lambda) .
$$

In the denominator appears the Fredholm determinant of the linear integral operator acting on the contour $\mathscr{C}$ with an integral kernel $\delta \Gamma[v](\lambda) / \delta v(\mu)_{\mid v=\gamma \rho}$. The contour $\mathscr{C}$ is defined in (D.11).

Proof-

We have, by definition, $\mathcal{L}_{\Gamma}(\gamma, z)=\lim _{s \rightarrow+\infty} \mathcal{L}_{s}(\gamma, z)$ with

$$
\mathcal{L}_{s}(\gamma, z)=\left.\sum_{n_{1}, \ldots, n_{s}} \prod_{r=1}^{s}\left\{\frac{1}{n_{r} !} \frac{\partial^{n_{r}}}{\partial \varsigma_{r}^{n_{r}}}\right\} \cdot \prod_{r=1}^{s} \Gamma^{n_{r}}\left[\gamma f_{s}\left(* \mid\left\{\varsigma_{p}\right\}\right)\right]\left(t_{r}\right) \cdot \mathcal{F}\left[\gamma f_{s}\left(* \mid\left\{\varsigma_{p}\right\}\right)\right](z)\right|_{\varsigma_{a}=0} .
$$

The above series representation for $\mathcal{L}_{s}(\gamma, z)$ corresponds to a particular case of a multidimensional Lagrange series.

We start by checking the convergence conditions. Let $C$ denote a common constant of regularity for the functionals $\mathcal{F}$ and $\Gamma$, ie for any $v(\lambda, \boldsymbol{y}) \in \mathscr{O}\left(M \times W_{y}\right)$, with $W_{y} \subset \mathbb{C}^{\ell_{y}}$ such that $\|v\|_{M \times W_{y}} \leq C$ one has

$$
\mathcal{F}[v(*, \boldsymbol{y})](\boldsymbol{z}) \in \mathscr{O}\left(W_{y} \times W_{z}\right) \quad \text { and } \quad \Gamma[v(*, \boldsymbol{y})](\lambda) \in \mathscr{O}\left(M \times W_{y}\right)
$$


Then let $r>0$ and $\gamma_{0}>0$ be as given by (D.14) but with $C_{\Gamma}$ being replaced with $C, s$ be large enough so that $\sum_{a=1}^{s}\left|t_{a}-t_{a+1}\right| \leq 2|\partial M|$ and $|\gamma| \leq 2 \gamma_{0}$. It then follows from (D.6) that $\left\|\gamma f_{s}\left(\cdot \mid\left\{\varsigma_{a}\right\}\right)\right\|_{K}<C$. It is also easy to see that for $\left|\varsigma_{p}\right| \leq r$ and for any $t_{p} \in \partial M$, one has $\left|\Gamma\left[\gamma f_{s}\left(* \mid\left\{\varsigma_{p}\right\}\right)\right]\left(t_{k}\right)\right| \leq r / 2$. Therefore,

- $\Gamma\left[\gamma f_{s}\left(* \mid\left\{\varsigma_{p}\right\}\right)\right]\left(t_{k}\right), k=1, \ldots, s$ and $\mathcal{F}\left[\gamma f_{s}\left(* \mid\left\{\varsigma_{p}\right\}\right)\right](z)$ are holomorphic functions of $\left\{\varsigma_{a}\right\}$ in $\mathcal{D}_{0, r}^{s}$;

- for $\left|\varsigma_{k}\right|=3 r / 4$ with $k \in \llbracket 1 ; s \rrbracket$ one has $\left|\Gamma\left[\gamma f_{s}\left(* \mid\left\{\varsigma_{p}\right\}\right)\right]\left(t_{k}\right)\right| \leq r / 2<3 r / 4$.

Hence, according to theorem D.1, the multidimensional Lagrange series is convergent and its sum is given by

$$
\mathcal{L}_{s}(\gamma, z)=\frac{\mathcal{F}\left[\gamma f_{s}\left(* \mid\left\{\tau_{p}\right\}\right)\right](z)}{\operatorname{det}_{s}\left[\delta_{j k}-\frac{\partial}{\partial \varsigma_{k}} \Gamma\left[\gamma f_{s}\left(* \mid\left\{\varsigma_{p}\right\}\right)\right]\left(t_{j}\right)\right]_{\mid S_{p}=\tau_{p}}}
$$

where $\left(\tau_{1}, \ldots, \tau_{s}\right)$ is the unique solution to the system $\tau_{j}=\Gamma\left[\gamma f_{s}\left(* \mid\left\{\tau_{p}\right\}\right)\right]\left(t_{j}\right)$ with $\left|\tau_{j}\right|<r$ for all $j$.

It is easy to see that, in fact, $\mathcal{L}_{s}(\gamma, \boldsymbol{z})$ is a uniform limit of holomorphic functions of $(\gamma, \boldsymbol{z}) \in \mathcal{D}_{0,2 \gamma_{0}} \times W_{z}$. Therefore, $\mathcal{L}_{s}(\gamma, z)$ is holomorphic on all compact subsets of $\mathcal{D}_{0,2 \gamma_{0}} \times W_{z}$. Moreover, there one can permute any partial $\gamma$ or $z$-derivatives with the summations in (D.19). It is also clear for the previously obtained bounds that, $\mathcal{L}_{s}(\gamma, z)$ is well defined for any $s$ large enough and this independently of the choice of the points $t_{k}$.

We now show that its $s \rightarrow+\infty$ limit exists and then we will compute it. It is readily inferred from the integral representation

$$
\tau_{j}=\int_{|\zeta a|=r} \frac{\zeta_{j}}{\prod_{p=1}^{s}\left\{\zeta_{p}-\Gamma\left[\gamma f_{s}\left(* \mid\left\{\zeta_{a}\right\}\right)\right]\left(t_{p}\right)\right\}} \mathrm{d}^{s} \zeta
$$

that $\tau_{j} \equiv \tau_{j}(\gamma), j=1, \ldots, s$, solving the system $\tau_{j}=\Gamma\left[\gamma f_{s}\left(* \mid\left\{\tau_{p}\right\}\right)\right]\left(t_{j}\right)$, is a holomorphic function of $\gamma$ for $|\gamma| \leq \gamma_{0}$. Hence, the function $\rho_{s}(\lambda ; \gamma)=\Gamma\left[\gamma f_{s}\left(* \mid\left\{\tau_{p}\right\}\right)\right](\lambda)$ is holomorphic in $(\lambda, \gamma) \in M \times \overline{\mathcal{D}}_{0, \gamma_{0}}$. Also, by construction,

$$
\rho_{s}\left(t_{j} ; \gamma\right)=\tau_{j}(\gamma) \quad \text { and } \quad\|\rho\|_{M \times \mathcal{D}_{0, \gamma_{0}}}<r
$$

Now let $\rho$ be the unique solution to $\rho(\lambda)=\Gamma[\gamma \rho+\gamma h](\lambda)$ with $\left\|\rho_{s}\right\|_{M} \leq r$, as follows from lemmaD.1

Then, keeping the $\gamma$ dependence implicit, we consider

$$
\rho(\lambda)-\rho_{s}(\lambda)=\Gamma[\gamma(\rho+h)](\lambda)-\Gamma\left[\gamma f_{s}\left(* \mid\left\{\rho\left(t_{p}\right)\right\}\right)\right](\lambda)+\underbrace{\Gamma\left[\gamma f_{s}\left(* \mid\left\{\rho\left(t_{p}\right)\right\}\right)\right](\lambda)-\Gamma\left[\gamma f_{s}\left(* \mid\left\{\rho_{s}\left(t_{p}\right)\right\}\right)\right](\lambda)}_{\psi_{s}(\lambda)} .
$$

It follows that

$$
\left\|\psi_{s}\right\|_{M} \leq C_{\Gamma}^{\prime} \gamma_{0}\left\|f_{s}\left(* \mid\left\{\rho\left(t_{p}\right)\right\}\right)-f_{s}\left(* \mid\left\{\rho_{s}\left(t_{p}\right)\right\}\right)\right\|_{K} \leq \frac{2 C_{\Gamma}^{\prime} \gamma_{0}|\partial M|}{2 \pi \mathrm{d}(\partial M, K)}\left\|\rho-\rho_{s}\right\|_{M}<\frac{1}{2}\left\|\rho-\rho_{s}\right\|_{M} .
$$

Hence, $\left\|\rho-\rho_{s}-\psi_{s}\right\|_{M} \geq\left\|\rho-\rho_{s}\right\|_{M} / 2$. On the other hand, it follows from (D.24) that

$$
\left\|\rho-\rho_{s}-\psi_{s}\right\|_{M}=\left\|\Gamma[\gamma \rho+\gamma h]-\Gamma\left[\gamma f_{s}\left(* \mid\left\{\rho\left(t_{p}\right)\right\}\right]\right]\right\|_{M} \leq \gamma_{0} C_{\Gamma}^{\prime}\left\|\rho+h-f_{s}\left(* \mid\left\{\rho\left(t_{p}\right)\right\}\right)\right\|_{K} \underset{s \rightarrow+\infty}{\longrightarrow} 0
$$

Therefore $\rho_{s}$ converges uniformly to $\rho$ on $M$. Hence, in virtue of the regularity of $\mathcal{F}$,

$$
\mathcal{F}\left[\gamma f_{s}\left(* \mid\left\{\rho_{s}\left(t_{p}\right)\right\}\right)\right](z) \underset{s \rightarrow+\infty}{\longrightarrow} \mathcal{F}[\gamma \rho](z) \quad \text { uniformly in }(\gamma, z) \in \overline{\mathcal{D}}_{0, \gamma_{0}} \times W_{z}
$$


It remains to compute the limit of the determinant. It follows from the functional derivative property (D.11) that

$$
\frac{\partial}{\partial \varsigma_{k}} \Gamma\left[\gamma f_{s}\left(* \mid\left\{\varsigma_{p}\right\}\right)\right]\left(t_{j}\right)_{\mid\left\{\varsigma_{p}\right\}=\left\{\tau_{p}\right\}}=\left.\gamma\left(t_{k+1}-t_{k}\right) \int_{\mathscr{C}} \frac{\mathrm{d} \mu}{2 i \pi} \frac{1}{t_{k}-\mu} \frac{\delta \Gamma}{\delta v(\mu)}\left[v+\gamma f_{s}\left(* \mid\left\{\tau_{p}\right\}\right)\right]\left(t_{j}\right)\right|_{v=0} .
$$

By expanding the determinant appearing in (D.21) into its discreet Fredholm series we get

$$
\operatorname{det}_{s}\left[\delta_{j k}-\frac{\partial}{\partial \varsigma_{k}} \Gamma\left[\gamma f_{s}\left(* \mid\left\{\varsigma_{p}\right\}\right)\right]\left(t_{j}\right)\right]_{\mid\left\{\varsigma_{p}\right\}=\left\{\tau_{p}\right\}}=\sum_{p=0}^{s} \frac{(-\gamma)^{p}}{p !} \int_{\mathscr{C}} \mathrm{d}^{p} \mu \operatorname{det}_{p}\left[A_{s}\left(\mu_{q}, \mu_{\ell}\right)\right]
$$

with

$$
\begin{aligned}
A_{s}\left(\mu_{q}, \mu_{\ell}\right)=\left.\sum_{k=1}^{s} \frac{t_{k+1}-t_{k}}{2 i \pi\left(t_{k}-\mu_{\ell}\right)} \frac{\delta}{\delta v\left(\mu_{q}\right)} \Gamma\left[v+f_{s}\left(* \mid\left\{\rho_{s}\left(t_{p}\right)\right\}\right)\right]\left(t_{j}\right)\right|_{v=0} \\
\qquad\left.\underset{s \rightarrow+\infty}{\longrightarrow} \int_{\partial M} \frac{\mathrm{d} \zeta}{2 i \pi} \frac{1}{\zeta-\mu_{\ell}} \frac{\delta \Gamma[v+\gamma \rho](\zeta)}{\delta v\left(\mu_{q}\right)}\right|_{v=0}=\left.\frac{\delta \Gamma[v+\gamma \rho]}{\delta v\left(\mu_{q}\right)}\left(\mu_{\ell}\right)\right|_{v=0}
\end{aligned}
$$

The above convergence is uniform in $\left(\mu_{q}, \mu_{\ell}\right) \in \mathscr{C} \times \mathscr{C}$. Therefore, by elementary estimates, we obtain that the determinant of interest does indeed converge to the Fredholm determinant given in $(\overline{D .18})$, this uniformly in $|\gamma| \leq \gamma_{0}$.

Therefore, we obtain that $\mathcal{L}_{S}(\gamma, z)$ is a sequence of holomorphic functions on $\mathcal{D}_{0, \gamma_{0}} \times \operatorname{Int}\left(W_{z}\right)$. that converges uniformly. As a consequence, $\mathcal{L}_{\Gamma}(\gamma, z)$ is holomorphic on every compact subset of $\mathcal{D}_{0, \gamma_{0}} \times \operatorname{Int}\left(W_{z}\right)$ and one can permute any partial- $\gamma$ or $z$ derivative of finite order with the $s \rightarrow+\infty$ limit on these compacts.

\section{D.5 Examples}

We now treat two examples that are of direct interest for the resummation of the form factor series. In the below examples, $\phi(\lambda, \mu)$ refers to the dressed phase (2.10). We remind that it is holomorphic on $U_{\delta} \times U_{\delta}$. In the following, the compacts $K$ and $M \subset U_{\delta}$ are such that $[-q ; q]$ is contained in their interior. We will also consider functions $h$ that are holomorphic on $M$.

\section{D.5.1 $\Gamma[\rho](\mu)$ as a linear functional of $\rho$}

Let $\Gamma[\rho](\lambda)=\int_{-q}^{q} \mathrm{~d} \mu \partial_{\lambda} \phi(\mu, \lambda) \rho(\mu)$. Then given a regular functional $\mathcal{F}[\cdot](z), z \in W_{z} \subset \mathbb{C}^{\ell_{z}}$, there exists $\gamma_{0}>0$ such that for $(\gamma, z) \in \overline{\mathcal{D}}_{0, \gamma_{0}} \times W_{z}$

$$
\mathcal{L}_{\Gamma}(\gamma, z)=\frac{\mathcal{F}[\rho](z)}{\operatorname{det}_{[-q ; q]}\left[I-\gamma \partial_{\lambda} \phi\right]} \quad \text { with } \quad \rho(\lambda)-\gamma \int_{-q}^{q} \partial_{\lambda} \phi(\mu, \lambda) \rho(\mu) \mathrm{d} \mu=h(\lambda)
$$

The limit defining $\mathcal{L}_{\Gamma}(\gamma, z)$ as in (D.18) is uniform in respect to such parameters $z$ and $|\gamma| \leq \gamma_{0}$.

Proof- 
In order to apply proposition D.2, one should check the assumptions on the functional $\Gamma$. It is readily seen that, independently of the norm of $v$ and $\rho$

$\|\Gamma[v]\|_{M} \leq 2 q\left\|\partial_{\lambda} \phi\right\|_{M \times M}\|v\|_{K} \quad$ and $\quad\|\Gamma[v]-\Gamma[\rho]\|_{M} \leq 2 q\left\|\partial_{\lambda} \phi\right\|_{M \times M}\|v-\rho\|_{K} \quad$ and $\quad \frac{\delta \Gamma[\tau]}{\delta \tau(\zeta)}(\mu)=\left(\partial_{\mu} \phi\right)(\zeta, \mu)$.

The validity of the holomorphicity conditions is readily checked by standard derivation under the integral theorems. One is thus in position to apply proposition D.2 and the claim follows.

\section{D.5.2 Non-linear functional $\Gamma^{(L)}[\rho](\mu)$}

We now treat the case of the non-linear functional below

$$
\Gamma^{(L)}[\rho](\mu)=\sum_{j \in J} \phi\left(\mu, \mu_{j}\right)-\phi\left(\mu, \lambda_{j}\right)=\sum_{j \in J} \oint_{\mathscr{C}_{q}} \phi(\mu, \omega)\left\{\frac{\xi^{\prime}(\omega)}{\xi(\omega)-j / L}-\frac{\xi_{\rho}^{\prime}(\omega)}{\xi_{\rho}(\omega)-j / L}\right\} \frac{\mathrm{d} \omega}{2 i \pi} .
$$

There $J=\llbracket 1 ; N \rrbracket \backslash\left\{i_{1}, \ldots, i_{n}\right\}, \xi$ is given by $(\underline{2.6}), \xi_{F}=\xi+F / L$ and $0 \leq j / L \leq D$ with $N / L \rightarrow D$. Finally, $\mathscr{C}_{q}$ is a small counterclockwise Jordan curve around $[-q ; q]$ such that $\operatorname{Int}(K) \supset \mathscr{C}_{q}$. Note that $\mu_{a}$, resp. $\lambda_{a}$, appearing in (D.32) stand for the unique solutions to $\xi\left(\mu_{a}\right)=a / L$, resp. to $\xi_{\rho}\left(\lambda_{a}\right)=a / L$.

Proposition D.3 Let $\mathcal{F}[\cdot](z), z \in W_{z} \subset \mathbb{C}^{\ell_{z}}$ be a regular functional and assume that $N, L$ are large (and such that $N / L \rightarrow D)$. Then, there exists $\gamma_{0}>0$ such that for $(\gamma, z) \in \mathcal{D}_{0, \gamma_{0}} \times W_{z}$ and $L$ large enough

$$
\mathcal{L}_{\Gamma^{(L)}}(\gamma, z)=\frac{\mathcal{F}\left[\gamma \rho^{(L)}\right](z)}{\operatorname{det}_{\mathscr{C}_{q}}\left[I-\gamma \frac{\delta \Gamma^{(L)}[v]}{\delta v(\zeta)}(\mu)\right]_{\nu=\gamma \rho^{(L)}}},
$$

where $\rho^{(L)}$ is the unique solution to $\rho^{(L)}(\lambda)=h(\lambda)+\Gamma^{(L)}\left[\gamma \rho^{(L)}\right](\lambda)$. This solution is such that $\rho^{(L)}(\lambda)=\rho(\lambda)+$ $\mathrm{O}\left(L^{-1}\right)$, where $\rho$ solves the linear integral equation $\left(I+\frac{\gamma R}{2 \pi}\right) \cdot \rho=h$ and the $\mathrm{O}\left(L^{-1}\right)$ is a holomorphic function of $\gamma$ and $\lambda$ and holds uniformly in $|\gamma| \leq \gamma_{0}$ and $\lambda \in U_{\delta}$. Finally,

$$
\left.\frac{\delta \Gamma^{(L)}[v]}{\delta v(\zeta)}(\mu)\right|_{\nu=\gamma \tau}=\frac{\left(\partial_{\zeta} \phi\right)(\mu, \zeta)}{2 i \pi L} \sum_{j \in J} \frac{1}{\xi_{\gamma \tau}(\zeta)-j / L} .
$$

Above $I+R / 2 \pi$ stands for the resolvent of the Lieb kernel acting on $[-q ; q]$. And one has

$$
\operatorname{det}_{\mathscr{C}_{q}}\left[I-\gamma \frac{\delta \Gamma^{(L)}[v]}{\delta v(\zeta)}(\mu)\right]_{v=\gamma \rho^{(L)}}=\operatorname{det}_{[-q ; q]}[I+\gamma R / 2 \pi]\left(1+\mathrm{O}\left(\frac{1}{L}\right)\right) .
$$

\section{Proof-}

In order to apply proposition D.2, we ought to check that $\Gamma^{(L)}$ satisfies to all the necessary conditions. For this we observe that

$$
\epsilon=\inf |\xi(\omega)-\lambda|>0 \quad \text { where the inf is taken for } \omega \in \mathscr{C}_{q}, \lambda \in[0 ; D] .
$$

Then, we choose a constant $C>0$ and consider $L$ large enough so that $C<\epsilon L / 2$. It then follows that the functions $\xi_{v}(\omega)-j / L$, for $j=1, \ldots, N$, have no zeroes on $\mathscr{C}_{q}$ and some immediate neighborhood thereof provided that 
$v(z, \boldsymbol{y}) \in \mathscr{O}\left(M \times W_{y}\right)$ with $W_{y} \subset \mathbb{C}^{\ell_{y}}$ and $\|v\|_{M \times W_{y}}<C$. It then follows by derivation under the integral sign theorems that $\Gamma^{(L)}[v(*, \boldsymbol{y})](\mu)$ is holomorphic in $(\mu, \boldsymbol{y}) \in M \times W_{y}$.

In order to establish bounds on $\Gamma^{(L)}[v(*, \boldsymbol{y})](\mu)$ for $v$ holomorphic and such that $\|v\|_{M \times W_{y}}<C$ it is convenient to represent

$$
\frac{\xi^{\prime}(\omega)}{\xi(\omega)-j / L}-\frac{\xi_{v}^{\prime}(\omega)}{\xi_{v}(\omega)-j / L}=-\frac{v^{\prime}(\omega)}{L(\xi(\omega)-j / L)}+\int_{0}^{v(\omega)} \frac{\mathrm{d} t}{L} \frac{\xi_{v}^{\prime}(\omega)}{\left(\xi_{t}(\omega)-j / L\right)^{2}} .
$$

As $\mathscr{C}_{q} \subset \operatorname{Int}(K)$, there exists a constant $c_{1}>0$ such that for any function $v$ holomorphic on $K$, one has $\left\|v^{\prime}\right\|_{\mathscr{C}_{q}} \leq$ $c_{1}\|v\|_{K}$. Also,

$\inf \left|\xi_{t}(\omega)-s\right|>\epsilon / 2 \quad$ where the inf is taken for $\omega \in \mathscr{C}_{q}, \quad s \in[0 ; D]$ and $|t|<\epsilon L / 2$.

Hence, for any $v \in \mathscr{O}\left(M \times W_{y}\right)$ such that $\|v\|_{K \times W_{y}}<C<\epsilon L / 2$

$$
\begin{aligned}
&\left|\Gamma^{(L)}[v(*, \boldsymbol{y})](\mu)\right| \leq\|\phi\|_{M \times M} \frac{|J|}{2 \pi}\left|\mathscr{C}_{q}\right|\left\{\frac{c_{1}}{L \epsilon}\|v\|_{K \times W_{y}}+\left\|\xi_{v}^{\prime}\right\|_{\mathscr{C}_{q} \times W_{y}} \cdot \frac{\|v\|_{K \times W_{y}}}{L(\epsilon / 2)^{2}}\right\} \\
& \leq\|\phi\|_{M \times M} \frac{N\left|\mathscr{C}_{q}\right|}{2 \pi L \epsilon} c_{1}\left\{1+\frac{4}{\epsilon}\left[\|\xi\|_{K}+\epsilon / 2\right]\right\}\|v\|_{K \times W_{y}} .
\end{aligned}
$$

This provides an estimate for the constant $C_{\Gamma^{(L)}}^{\prime}$ entering in the bounds for $\left\|\Gamma^{(L)}[v]\right\|_{M \times W_{y}}$. Next one has

$$
\Gamma^{(L)}[\rho](\mu)-\Gamma^{(L)}[\tau](\mu)=\sum_{j \in J} \oint_{\mathscr{C}_{q}} \phi(\mu, \omega)\left\{\frac{\left(\tau^{\prime}-\rho^{\prime}\right)(\omega)}{\xi_{\tau}(\omega)-j / L}+\frac{(\rho-\tau)(\omega) \xi_{\tau}^{\prime}(\omega)}{\left(\xi_{\tau}(\omega)-j / L\right)^{2}}\right\} \frac{\mathrm{d} \omega}{2 i \pi L}+\mathcal{R}^{(L)}(\mu)
$$

Where,

$$
\mathcal{R}^{(L)}(\mu)=\sum_{j \in J} \oint_{\mathscr{C}_{q}} \phi(\mu, \omega)\left\{\frac{(\rho-\tau)(\omega)(\rho-\tau)^{\prime}(\omega)}{L^{2}\left(\xi_{\tau}(\omega)-j / L\right)^{2}}+2 \int_{\tau(\omega)}^{\rho(\omega)} \frac{\mathrm{d} t}{L^{2}} \frac{(t-\rho(\omega)) \xi_{\rho}^{\prime}(\omega)}{\left(\xi_{t}(\omega)-j / L\right)^{3}}\right\} \frac{\mathrm{d} \omega}{2 i \pi}=\mathrm{O}\left(\|\rho-\tau\|_{K}^{2}\right),
$$

this uniformly in $\mu \in M$ and $L$ large enough. Therefore,

$$
\left.\frac{\delta \Gamma^{(L)}[\gamma v](\mu)}{\delta \nu(\zeta)}\right|_{\nu=\gamma \rho}=\frac{\gamma}{2 i \pi L}\left(\partial_{\zeta} \phi\right)(\mu, \zeta) \sum_{j \in J} \frac{1}{\xi_{\gamma \rho}(\zeta)-j / L}
$$

It follows that there exists a sufficiently small open neighborhood $\mathcal{V}\left(\mathscr{C}_{q}\right)$ of $\mathscr{C}_{q}$ such that the functional derivative is holomorphic in $(\mu, \zeta) \in M \times \mathcal{V}\left(\mathscr{C}_{q}\right)$. Moreover, we get that there exists an $L$-independent constant $\widetilde{C}_{2}$ such that $\left\|\Gamma^{(L)}[\rho]-\Gamma^{(L)}[\tau]\right\|_{M} \leq \widetilde{C}_{2}\|\rho-\tau\|_{K}$

We are now in position to apply proposition D.2 It follows that $\mathcal{L}_{\Gamma^{(L)}}$ can be expressed in terms of the unique solution $\rho^{(L)}$ to $\rho^{(L)}(\mu)=h(\mu)+\Gamma^{(L)}\left[\gamma \rho^{(L)}\right](\mu)$ with $\left\|\rho^{(L)}\right\|_{M}<r$ uniformly in $|\gamma| \leq \gamma_{0}$.

This means that,

$$
\left.\frac{\delta \Gamma^{(L)}[\gamma \nu](\mu)}{\delta v(\zeta)}\right|_{\nu=\rho^{(L)}} \underset{L \rightarrow+\infty}{\longrightarrow} \gamma\left(\partial_{\zeta} \phi\right)(\mu, \zeta) \int_{-q}^{q} \frac{\mathrm{d} u}{2 i \pi} \frac{\xi^{\prime}(u)}{\xi(\zeta)-\xi(u)} \quad \text { uniformly in } \quad(\mu, \zeta) \in \mathscr{C}_{q}^{2} .
$$


In this limit, the contour integral $\mathscr{C}_{q}$ in the Fredholm determinant can be computed and since the Fredholm determinant of a trace class operator is continuous in respect to the trace class norm (which is bounded by the sup norm in the case of integral operators acting on compact contours)

$$
\operatorname{det}_{\mathscr{C}_{q}}\left[I-\delta \Gamma^{(L)}[\gamma \nu](\mu) /\left.\delta \nu(\zeta)\right|_{\nu=\rho^{(L)}}\right] \underset{L \rightarrow+\infty}{\rightarrow} \operatorname{det}_{[-q ; q]}\left[I-\gamma \partial_{\lambda} \phi(\mu, \lambda)\right]=\operatorname{det}_{[-q ; q]}[I+\gamma R / 2 \pi] .
$$

Where $R$ is the resolvent of the Lieb kernel.

We now characterize the leading behavior of the solution $\rho^{(L)}$ when $N, L \rightarrow+\infty$. By repeating the type of manipulations carried our previously, and using that $\rho^{(L)}$ is bounded on $K$ uniformly in $L$, we get that the nonlinear integral equation for $\rho^{(L)}$ takes the form

$$
\rho^{(L)}(\mu)=h(\mu)+\frac{\gamma}{L} \sum_{j \in J} \oint_{\mathscr{C}_{q}} \frac{\mathrm{d} \omega}{2 i \pi} \rho^{(L)}(\omega) \frac{\left(\partial_{\omega} \phi\right)(\mu, \omega)}{\xi(\omega)-j / L}+\mathrm{O}\left(\frac{1}{L}\right) .
$$

There the $\mathrm{O}$ is uniform in $\mu \in U_{\delta}$. The Riemann sum can be estimated by using the Euler-McLaurin formula and the uniform boundedness of $\rho^{(L)}$ on $K$. After carrying out the resulting contour integral over $\mathscr{C}_{q}$ we obtain

$$
\rho^{(L)}(\mu)=h(\mu)-\gamma \int_{-q}^{q} \frac{\mathrm{d} s}{2 \pi} R(\mu, s) \rho^{(L)}(s)+\mathrm{O}\left(L^{-1}\right) .
$$

The $\mathrm{O}$ appearing in (D.45) is holomorphic in $\mu \in U_{\delta}$. Indeed, $\rho^{(L)}$ just as all the other terms in (D.45) are holomorphic on $U_{\delta}$. This proves that $\rho^{(L)}$ admits an asymptotic expansion in $L$ such that $\rho^{(L)}(\omega)=\rho(\omega)+\mathrm{O}\left(L^{-1}\right)$, where $\rho$ is the solution to the integral equation $(I+\gamma R / 2 \pi) \cdot \rho=h$. As $\rho^{(L)}$ and $\rho$ are both holomorphic in $U_{\delta}$, so is the remainder. Moreover, one can convince oneself that this $\mathrm{O}$ is uniform in $\mu \in U_{\delta}$. Therefore, the regularity of the functional $\mathcal{F}[\cdot](z)(4.14)$ leads to $\mathcal{F}\left[\gamma \rho^{(L)}\right](z)=\mathcal{F}[\gamma \rho](z)+\mathrm{O}\left(L^{-1}\right)$.

\section{References}

[1] I.A. Aizenberg and A.P. Yuzhakov, Integral representations and residues in multidimensional complex analysis, American Mathematical Society.

[2] A. Berkovich and G. Murthy, "Time-dependent multi-point correlation functions of the non-linear Schrödinger model", Phys.Lett. A142 (1989), 121-127.

[3] H.W.J. Blöte, J.L. Cardy, and M.P. Nightingale, "Conformal invariance, the central charge, and universal finite-size amplitudes at criticality.", Phys. Rev. Lett. 56 (1986), 742-745.

[4] N. M. Bogoliubov, A. G. Izergin, and V. E. Korepin, "Quantum Inverse Scattering Method, Correlation Functions and Algebraic Bethe Ansatz.", Cambridge monographs on mathematical physics, 1993.

[5] N.M. Bogoliubov, A.G. Izergin, and V.E. Korepin, "Finite-size effects and critical indices of $1 D$ quantum models.", Pisma ZhETF 44 (1986), 405-407.

[6] H.E. Boos, M. Jimbo, T. Miwa, F. Smirnov, and Y. Takeyama, "Fermionic basis for space of states of operators in the XXZ model.", ArXiV hep-th/ 0702086.

[7] _ "Algebraic representation of correlation functions in integrable spin chains.", Ann. H. Poincare 7 (2006), 1395-1428. 
[8] , "Hidden Grassmann structure in the XXZ model.", Comm. Math. Phys. 272 (2007), 263-281.

[9] _ _ "Hidden Grassmann structure in the XXZ model II: creation operators.", Comm. Math. Phys. 286 (2009), 875-932.

[10] H.E. Boos and V.E. Korepin, "Evaluation of integrals representing correlations in XXX Heisenberg spin chains.", Math. Phys. Odyssey 2001: Integrable models and beyond 23 (2002), 65-108.

[11] J.L. Cardy, "Conformal invariance and universality in finite-size scaling.", J. Phys. A: Math. Gen. 17 (1984), L385-387.

[12] J.-S. Caux, L. I. Glazman, A. Imambekov, and A. Shashi, "Exact asymptotes of static and dynamic correlation functions of the 1D Bose gas.", arXiv:1010.2268 (2007).

[13] H. Cheng and T. T. Wu, "Theory of Toeplitz determinants and the spin correlations functions of the two dimensionnal Ising model III.", Phys. Rev. 164 (1967), 719-735.

[14] T. Deguchi and C. Matsui, "Form factors of integrable higher-spin XXZ chains and the affine quantum-group symmetry.", Nucl. Phys. B 814 (2009), 409-438.

[15] _ "Correlation functions of the integrable higher-spin XXX and XXZ spin chains through the fusion method.", Nucl. Phys. B 831 (2010), 389-407.

[16] P.A. Deift and X. Zhou, "A steepest descent method for oscillatory Riemann-Hilbert problems. Asymptotics of the mKdV equation.", Ann. Of. Math. 137 (1993), 297-370.

[17] C. Destri and H. J. DeVega, "New thermodynamic Bethe Ansatz equations without strings.", Phys. Rev. Lett 69 (1992), 2313-2317.

[18] _ _ "Unified approach to Thermodynamic Bethe Ansatz and finite size corrections for lattice models and field theories.", Nucl. Phys. B 438 (1995), 413-454.

[19] T.C. Dorlas, "Orthogonality and completeness of the Bethe Ansatz eigenstates of the nonlinear Schrödinger model.", Comm. Math. Phys. 154, 2 (1993), 347-376.

[20] L.D. Faddeev, "How Algebraic Bethe Ansatz works for integrable model.", Les Houches lectures (1996).

[21] M.E. Fisher, "Quantum corrections to critical-point behavior.", Phys. Rev. Lett. 16 (1966), 11-14.

[22] M. Girardeau, "Relationship between Systems of Impenetrable Bosons and Fermions in One Dimension.", J. Math. Phys. 1 (1960), 516-523.

[23] F. Göhmann, A. Klümper, and A. Seel, "Integral representations for correlation functions of the XXZ chain at finite temperature.", J. Phys. A: Math. Gen. 37 (2004), 7625-7652.

[24] R.B. Griffiths, "Dependence of critical indicies on a parameter.", Phys. Rev. Lett. 24 (1970), 1479-1482.

[25] F.D.M. Haldane, "Demonstration of the "Luttinger liquid" character of Bethe-ansatz soluble models of 1-D quantum fluids", Phys. Lett. A 81 (1981), 153-155.

[26] _ "Effective harmonic fluid approach to low-energy properties of one-dimensional quantum fluids.", Phys. Rev. Lett. 47 (1981), 1840-1843. 
[27] "Luttinger liquid theory of one-dimensional quantum fluids: I. Properties of the Luttinger model and their extension to the general 1D interacting spinless Fermi gas", J. Phys. C: Solid State Phys. 14 (1981), 2585-2609.

[28] A. Imambekov and L.I. Glazman, "Exact Exponents of Edge Singularities in Dynamic Correlation Functions of $1 D$ Bose Gas.", Phys. Rev. Lett., 100, (2008), 206805.

[29] A.R. Its, A.G. Izergin, and V.E. Korepin, "Long-distance asymptotics of temperature correlators of the impenetrable Bose gas.", Comm. Math. Phys. 130 (1990), 471-488.

[30] A.R. Its, A.G. Izergin, V.E. Korepin, and N.A. Slavonv, "Differential equations for quantum correlation functions.", Int. J. Mod. Physics B4 (1990), 1003-1037.

[31] _ _ , "Temperature correlations of quantum spins.", Phys. Rev. Lett. 70 (1993), 1704.

[32] A.R. Its, A.G. Izergin, V.E. Korepin, and G.G. Varguzin, "Large-time and distance asymptotics of the correlator of the impenetrable bosons at finite temperature.", Physica D 54 (1991), 351.

[33] A.R. Its and N.A. Slavonv, "On the Riemann-Hilbert approach to the asymptotic analysis of the correlation functions of the Quantum Nonlinear Schrdinger equation. Non-free fermion case.", Int. J. Mod. Physics B4 (1990), 1003-1037.

[34] A.G. Izergin and V.E. Korepin, "Lattice versions of quantum field theory models in two dimensions.", Nucl. Phys. B 205 (1982), 401-413.

[35] _ , "The quantum inverse scattering method approach to correlation functions.", Comm. Math. Phys. 94 (1984), 67-92.

[36] _ "Correlation functions for the Heisenberg XXZ antiferromagnet.", Comm. Math. Phys. 99 (1985), 271-302.

[37] N.P. Hasenclever J. Damerau, F. Gohmann and A. Klumper, "Density matrices for finite segments of Heisenberg chains of arbitrary length.", J. Phys. A: Math. Theor. 40 (2007), 4439-4453.

[38] M. Jimbo, R. Kedem, T. Kojima, H. Konno, and T. Miwa, "XXZ chain with a boundary.", Nucl. Phys. B 441 (1995), 437-470.

[39] M. Jimbo, K. Miki, T. Miwa, and A. Nakayashiki, "Correlation functions of the XXZ model for $\Delta<-1 . "$, Phys. Lett. A 168 (1992), 256-263.

[40] M. Jimbo and T. Miwa, "QKZ equation with $|q|=1$ and correlation functions of the XXZ model in the gapless regime.", J. Phys. A 29 (1996), 2923-2958.

[41] M. Jimbo, T. Miwa, and F. Smirnov, "Hidden Grassman structure in the XXZ model V: sine-Gordon model.".

[42] __ "On one-point functions of descendants in sine-Gordon model.".

[43] N. Kitanine, K.K. Kozlowski, J.-M. Maillet, N.A. Slavnov, and V. Terras, "Form factor approach ot the long-distance asymptotic behavior of correlation functions in critical models.", to appear.

[44] __ , "Thermodynamic limit of particle-hole form factors in the massless XXZ Heisenberg chain. ", mathph: 1003.4557. 
[45] "Algebraic Bethe Ansatz approach to the asymptotics behavior of correlation functions.", J. Stat. Mech: Th. and Exp. 04 (2009), P04003.

[46] _ , "On the thermodynamic limit of form factors in the massless XXZ Heisenberg chain.", J. Math. Phys. 50 (2009), 095209.

[47] N. Kitanine, J.-M. Maillet, N.A. Slavnov, and V. Terras, "Emptiness formation probability of the XXZ spin1/2 Heisenberg chain at Delta=1/2.", J. Phys. A: Math. Gen. 35 (2002), L385-L391.

[48] _ "Spin-spin correlation functions of the XXZ-1/2 Heisenberg chain in a magnetic field.", Nucl.Phys. B 641 (2002), 487-518.

[49] _ _ "Dynamical correlation functions of the XXZ spin-1/2 chain.", Nucl.Phys. B 729 (2005), 558-580.

[50] _ "Exact results for the sigma-z two-point function of the XXZ chain at Delta=1/2.", J. Stat. Mech. 0509 (2005), L002.

[51] _ , "Master equation for spin-spin correlation functions of the XXZ chain.", Nucl.Phys. B 712 (2005), $600-622$.

[52] N. Kitanine, J.-M. Maillet, and V. Terras, "Correlation functions of the XXZ Heisenberg spin-1/2 chain in a magnetic field.", Nucl. Phys. B 567 (2000), 554-582.

[53] _ _ "Form factors of the XXZ Heisenberg spin-1/2 finite chain.", J. Phys. A: Math. Gen. 35 (2002), L753-10502.

[54] A. Klümper, M. T. Batchelor, and P. A. Pearce, "Central charges for the 6- and 19-vertex models with twisted boundary conditions.", J. Phys. A: Math. Gen. 24 (1991), 3111-3133.

[55] A. Klümper, T. Wehner, and J. Zittartz, "Conformal spectrum of the 6-vertex model.", J. Phys. A: Math. Gen. 26 (1993), 2815-2827.

[56] T. Kojima, V.E. Korepin, and N.A. Slavonv, "Determinant representation for dynamical correlation functions of the quantum nonlinear Schrdinger equation.", Comm. Math. Phys. 188 (1997), 657-689.

[57] V. E. Korepin and N. A. Slavnov, "Correlation functions of fields in one-dimensional Bose gas.", Comm. Math. Phys. 136 (1991), 633-644.

[58] _ , "The new identity for the scattering matrix of exactly solvable models.", Eur. Phys. J. B 5 (1998), $555-557$.

[59] V.E. Korepin, "Dual field formulation of quantum integrable models.", Comm.Math.Phys. 113 (1987), 177190.

[60] V.E. Korepin and N.A. Slavnov, "The time dependent correlation function of an impenetrable Bose gas as a Fredholm minor I.", Comm. Math.Phys. 129 (1990), 103-113.

[61] _ _ "The form factors in the finite volume.", Int.J.Mod.Phys.B 13 (1999), 2933-2942.

[62] K. K. Kozlowski, Riemann-Hilbert approach to the time-dependent generalized sine kernel.", math-ph: 10115897. 
[63] K. K. Kozlowski, J.-M. Maillet, and N. A. Slavnov, "Long-distance behavior of temperature correlation functions of the quantum one-dimensional Bose gas.", math-ph: 10113149.

[64] K.K. Kozlowski, "On Form Factors of the conjugated field in the non-linear Schrödinger model.", to appear.

[65] K.K. Kozlowski and V. Terras, "Long-time and large-distance asymptotic behavior of the current-current correlators in the non-linear Schrödinger model.", math-ph:11010844.

[66] E.H. Lieb and W. Liniger, "Exact Analysis of an Interacting Bose Gas. I. The General Solution and the Ground state.", Phys. Rev. 130 (1963), 1605-1616.

[67] A. Luther and I. Peschel, "Calculation of critical exponents in two dimensions from quantum field theory in one dimension.", Phys. Rev. B 12(9) (1975), 3908-3917.

[68] B. M. McCoy, J. H. H. Perk, and R. E. Shrock, "Correlation functions of the transverse Ising chain at the critical field for large temporal and spacial separation.", Nucl. Phys. B 220 (1983), 269-282.

[69] _ , "Time-dependent correlation functions of the transverse Ising chain at the critical magnetic field.", Nucl. Phys. B 220 (1983), 35-47.

[70] B. M. McCoy and T. T. Wu, "Theory of Toeplitz determinants and the spin correlations functions of the two dimensionnal Ising model IV.", Phys. Rev. 162 (1967), 436-475.

[71] _ , "The two-dimensional Ising model.", lecture notes in physics vol 246, Harvard University Press, Cambridge, Massachusetts, 1973.

[72] G. Müller and R. E. Shrock, "Dynamic correlation functions for one-dimensional quantum-spin systems: new results based on a rigorous approach.", Phys. Rev. B 29 (1984), 288-301.

[73] T. Oota, "Quantum Projectors and Local Operators in Lattice Integrable Models.", J. Phys. A: Math. Gen. 37 (2004), 441-452.

[74] A. M. Polyakov, "Conformal symmetry of critical fluctuations.", Pis. ZhETP 12 (1970), 538-541.

[75] J. Sato, M. Shiroishi, and M. Takahashi, "Exact evaluation of density matrix elements for the Heisenberg chain.", J. Stat. Mech. (2006), P12017.

[76] B. Simons, "Notes on infinite dimensional determinants.", Adv. Math. 24 (1977), 244-273.

[77] E.K. Sklyanin, "Quantum version of the method of inverse scattering problem.", J. Math. Sci. 19 (1982), 1547-1596.

[78] N.A. Slavnov, "Non-equal time current correlation function in a one-dimensional Bose gas.", Theor. Math. Phys. 82 (1990), 273-282.

[79] __ "A nonlinear identity for the scattering phase of integrable models.", Theor. Math. Phys. 116 (1998), $362-366$.

[80] _ "Integral equations for the correlation functions of the quantum one-dimensional Bose gas. ", Theor. Math. Phys. 121 (1999), 1358-1376.

[81] F. A. Smirnov, "Form Factors in completely intergrable models of quantum field theory.", Advanced Series in Mathematical Physics, vol. 14, World Scientific, 1992. 
[82] F.A. Smirnov, "The general formula for solitons form factors in sine-Gordon model.", J. Phys. A 19 (1986), L575.

[83] C. A. Tracy and H. Vaidya, "One particle reduced density matrix of impenetrable bosons in one dimension at zero temperature.", J. Math. Phys. 20 (1979), 2291-2312.

[84] F. Woynarowich, "Excitation spectrum of the spin-1/2 Heisenberg chain and conformal invariance.", Phys. Rev. Lett. 59 (1987), 259-261.

[85] C. N. Yang and C. P. Yang, "Thermodynamics of a one-dimensional system of bosons with repulsive deltainteractions.", J. Math. Phys. 10 (1969), 1115-1122. 